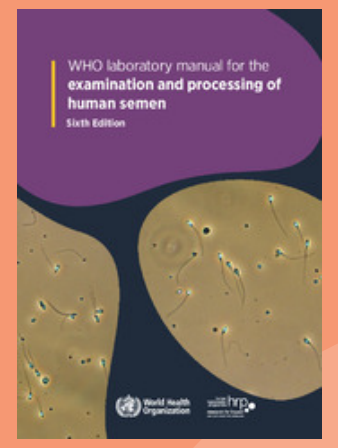

This manual has been superseded by the publication entitled:

WHO laboratory manual for the examination and processing of human semen, 6th ed, which can be found at:

https://apps. who.int/iris/handle/10665/343208

\title{
WHO laboratory manual for the Examination and processing of human semen
}

FIFTH EDITION

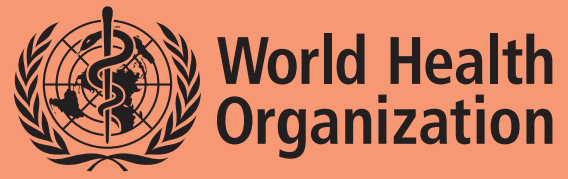




\section{WHO laboratory manual for the Examination and processing of human semen}

FIFTH EDITION

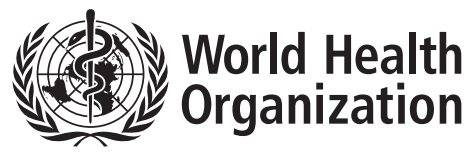


WHO Library Cataloguing-in-Publication Data

WHO laboratory manual for the examination and processing of human semen - 5th ed.

Previous editions had different title: WHO laboratory manual for the examination of human semen and sperm-cervical mucus interaction.

1.Semen - chemistry. 2.Semen - laboratory manuals. 3.Spermatozoa - laboratory manuals. 4.Sperm count. 5.Sperm-ovum interactions - laboratory manuals. 6.Laboratory techniques and procedures - standards. 7.Quality control. I.World Health Organization.

ISBN 9789241547789

(NLM classification: QY 190)

\section{(c) World Health Organization 2010}

All rights reserved. Publications of the World Health Organization can be obtained from WHO Press, World Health Organization, 20 Avenue Appia, 1211 Geneva 27, Switzerland (tel.: +41 22791 3264; fax: +41 22791 4857; e-mail: bookorders@who.int). Requests for permission to reproduce or translate WHO publicationswhether for sale or for noncommercial distribution-should be addressed to WHO Press, at the above address (fax: +41 22791 4806; e-mail: permissions @who.int).

The designations employed and the presentation of the material in this publication do not imply the expression of any opinion whatsoever on the part of the World Health Organization concerning the legal status of any country, territory, city or area or of its authorities, or concerning the delimitation of its frontiers or boundaries. Dotted lines on maps represent approximate border lines for which there may not yet be full agreement.

The mention of specific companies or of certain manufacturers' products does not imply that they are endorsed or recommended by the World Health Organization in preference to others of a similar nature that are not mentioned. Errors and omissions excepted, the names of proprietary products are distinguished by initial capital letters.

All reasonable precautions have been taken by the World Health Organization to verify the information contained in this publication. However, the published material is being distributed without warranty of any kind, either expressed or implied. The responsibility for the interpretation and use of the material lies with the reader. In no event shall the World Health Organization be liable for damages arising from its use.

Printed in Switzerland.

Cover photo: courtesy of C. Brazil 


\section{CONTENTS}

Acknowledgements

Acronyms and abbreviations used in this manual

$\mathrm{xi}$

\begin{tabular}{rlr} 
Chapter 1 Background & 1 \\
\hline 11 & Introduction
\end{tabular}

$\begin{array}{lll}1.1 & \text { Introduction } & 1 \\ 1.2 & \text { The fifth edition } & 1\end{array}$

1.3 Scope of the manual 3

PART I. SEMEN ANALYSIS

\begin{tabular}{rlr} 
Chapter 2 & Standard procedures & 7 \\
\hline 2.1 & Introduction & 7
\end{tabular}

2.2 Sample collection $\quad 10$

2.2.1 Preparation 10

2.2.2 Collection of semen for diagnostic or research purposes 11

2.2.3 Sterile collection of semen for assisted reproduction 11

2.2.4 Sterile collection of semen for microbiological analysis $\quad 11$

2.2.5 Collection of semen at home 12

2.2.6 Collection of semen by condom 12

2.2.7 Safe handling of specimens 13

$\begin{array}{lll}2.3 & \text { Initial macroscopic examination } & 13\end{array}$

2.3.1 Liquefaction 13

2.3.2 Semen viscosity 14

2.3.3 Appearance of the ejaculate 15

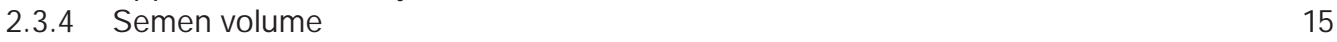

2.3.5 Semen $\mathrm{pH} \quad 16$

2.4 Initial microscopic investigation 17

2.4.1 Thorough mixing and representative sampling of semen $\quad 17$

2.4.2 Making a wet preparation 18

2.4.3 Aggregation of spermatozoa 19

2.4.4 Agglutination of spermatozoa $\quad 19$

2.4.5 Cellular elements other than spermatozoa 21

2.5 Sperm motility 21

2.5.1 Categories of sperm movement 22

2.5.2 Preparing and assessing a sample for motility 22

2.5.3 Worked examples 25

$\begin{array}{ll}2.5 .4 & \text { Lower reference limit }\end{array}$

2.6 Sperm vitality 26

2.6.1 Vitality test using eosin-nigrosin 27

2.6.2 Vitality test using eosin alone $\quad 29$

2.6.3 Vitality test using hypo-osmotic swelling 30

2.7 Sperm numbers $\quad 32$

2.7.1 Types of counting chamber 34

2.7.2 The improved Neubauer haemocytometer 34

2.7.3 Using the haemocytometer grid 35

2.7.4 Care of the counting chamber 35

2.7.5 Fixative for diluting semen 36

2.7.6 Importance of counting sufficient spermatozoa 36 
2.8 Routine counting procedure 37

2.8.1 Determining the required dilution 38

2.8.2 Preparing the dilutions and loading the haemocytometer chambers 39

2.8.3 Assessing sperm numbers in the counting chambers 41

2.8.4 Calculation of the concentration of spermatozoa in semen 43

2.8.5 Worked examples 43

2.8.6 Lower reference limit for sperm concentration 44

2.8.7 Calculation of the total number of spermatozoa in the ejaculate 44

2.8.8 Lower reference limit for total sperm number 44

2.9 Low sperm numbers: cryptozoospermia and suspected azoospermia $\quad 45$

2.10 When an accurate assessment of low sperm numbers is not required 45

2.10.1 Taking no further action $\quad 45$

2.10.2 Examination of centrifuged samples to detect spermatozoa 45

2.10.3 Examination of non-centrifuged samples to detect motile spermatozoa 46

2.11 When an accurate assessment of low sperm numbers is required 48

2.11.1 Assessing low sperm numbers in the entire improved Neubauer chamber
(phase-contrast microscopy)

2.11.2 Assessing low sperm numbers in large-volume disposable chambers

2.12 Counting of cells other than spermatozoa $\quad 55$

2.12.1 Calculation of the concentration of round cells in semen 55

2.12.2 Sensitivity of the method 56

$\begin{array}{lll}2.12 .3 & \text { Worked examples } & 56\end{array}$

2.13 Sperm morphology $\quad 56$

2.13.1 The concept of normal spermatozoa

2.13.2 Preparation of semen smears 58

2.14 Staining methods $\quad 62$

2.14.1 Traditional fixation and sequential staining 62

2.14.2 Papanicolaou staining procedure for sperm morphology 63

2.14.3 Shorr staining procedure for sperm morphology 65

2.14.4 Rapid staining procedure for sperm morphology 66

2.15 Examining the stained preparation 67

2.15.1 Classification of normal sperm morphology 67

2.15.2 Classification of abnormal sperm morphology 69

2.16 Morphology plates $\quad 70$

2.17 Analysing a sperm morphology smear $\quad 99$

2.17.1 Assessment of normal sperm morphology 99

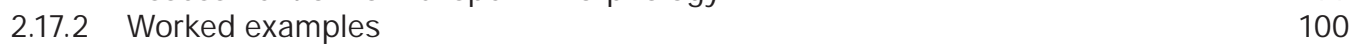

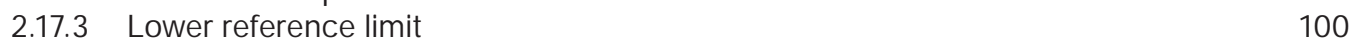

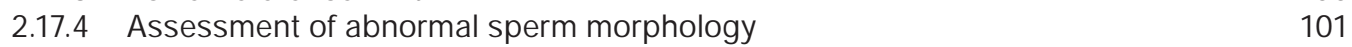

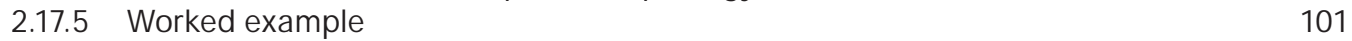

$\begin{array}{ll}2.17 .6 & \text { Assessment of specific sperm defects } \\ & 102\end{array}$

2.18 Assessment of leukocytes in semen $\quad 102$

2.18.1 Staining cellular peroxidase using ortho-toluidine 103

2.19 Assessment of immature germ cells in semen 107

$\begin{array}{ll}2.20 & \text { Testing for antibody coating of spermatozoa } \\ 2.108\end{array}$

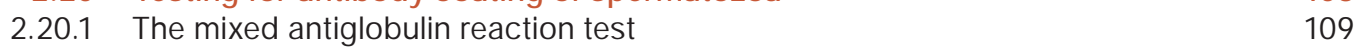

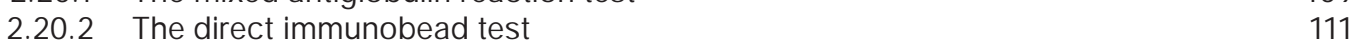

$\begin{array}{lll}2.20 .3 & \text { The indirect immunobead test } & 113\end{array}$ 
\begin{tabular}{lll} 
Chapter 3 & Optional procedures & 115 \\
\hline
\end{tabular}

3.1 Indices of multiple sperm defects 115

3.1.1 Calculation of indices of multiple morphological defects $\quad 115$

3.1.2 Worked example 116

3.2 Panleukocyte (C D 45) immunocytochemical staining 117

$\begin{array}{lll}3.2 .1 & \text { Principle } & 117\end{array}$

$\begin{array}{lll}3.2 .2 & \text { Reagents } & 118\end{array}$

$\begin{array}{lll}3.2 .3 & \text { Procedure } & 118\end{array}$

3.3 Interaction between spermatozoa and cervical mucus 122

3.3.1 In-vivo (postcoital) test 122

$\begin{array}{lll}\text { 3.3.2 In-vitro tests } & 125\end{array}$

3.3.3 In-vitro simplified slide test $\quad 126$

$\begin{array}{ll}\text { 3.3.4 Capillary tube test } & 127\end{array}$

3.4 Biochemical assays for accessory sex organ function $\quad 130$

3.4.1 Measurement of zinc in seminal plasma $\quad 130$

3.4.2 Measurement of fructose in seminal plasma $\quad 132$

3.4.3 Measurement of neutral $\alpha$-glucosidase in seminal plasma $\quad 134$

3.5 Computer-aided sperm analysis $\quad 136$

$\begin{array}{lll}3.5 .1 & \text { Introduction } & 136\end{array}$

3.5.2 Use of CASA to assess sperm motility 137

3.5.3 Use of CASA to estimate sperm concentration $\quad 140$

3.5.4 Computer-aided sperm morphometric assessment $\quad 140$

\begin{tabular}{lll} 
Chapter 4 & Research procedures & 142 \\
\hline
\end{tabular}

$\begin{array}{llr}4.1 & \text { Reactive oxygen species } & 142\end{array}$

$\begin{array}{lll}4.1 .1 & \text { Introduction } & 142\end{array}$

4.1.2 Measurement of reactive oxygen species generated by sperm suspensions 143

4.2 Human sperm-oocyte interaction tests 146

4.3 Human zona pellucida binding tests 146

4.4 Assessment of the acrosome reaction 147

4.4.1 Procedure for the fluorescence assessment of acrosomal status 147

$\begin{array}{ll}\text { 4.4.2 Induced acrosome reaction assay } & 150\end{array}$

4.5 Zona-free hamster oocyte penetration test 152

$\begin{array}{lll}4.5 .1 & \text { Protocol } & 152\end{array}$

$\begin{array}{ll}\text { 4.6 Assessment of sperm chromatin } & 157\end{array}$

\section{PART II. SPERM PRE PARATION}

\begin{tabular}{rlr} 
Chapter 5 & Sperm preparation techniques & 161 \\
\hline 5.1 & Introduction & 161 \\
5.1 .1 & When spermatozoa may need to be separated from seminal plasma & 161 \\
5.1 .2 & Choice of method & 161 \\
5.1 .3 & Efficiency of sperm separation from seminal plasma and infectious organisms & 162 \\
5.2 & General principles & 162 \\
5.3 & Simple washing & 163 \\
5.3 .1 & Reagents & 163 \\
5.3 .2 & Procedure & 163 \\
5.4 & Direct swim-up & 164 \\
5.4 .1 & Reagents & 164 \\
5.4 .2 & Procedure & 164
\end{tabular}


5.5 Discontinuous density gradients $\quad 165$

$\begin{array}{lll}\text { 5.5.1 Reagents } & 165\end{array}$

$\begin{array}{lll}5.5 .2 & \text { Procedure } & 166\end{array}$

5.6 Preparing HIV-infected semen samples 166

5.7 Preparing testicular and epididymal spermatozoa 167

5.7.1 Enzymatic method 167

5.7.2 Mechanical method 167

5.7.3 Processing sperm suspensions for intracytoplasmic sperm injection 167

5.8 Preparing retrograde ejaculation samples 168

$\begin{array}{lll}5.9 & \text { Preparing assisted ejaculation samples } & 168\end{array}$

\begin{tabular}{llr} 
Chapter 6 & Cryopreservation of spermatozoa & 169 \\
\hline
\end{tabular}

$\begin{array}{lll}6.1 & \text { Introduction } & 169\end{array}$

6.2 Semen cryopreservation protocols 172

6.2.1 Standard procedure $\quad 172$

6.2.2 Modified freezing protocols for oligozoospermia and surgically retrieved
spermatozoa

$\begin{array}{ll}\text { 6.2.3 Labelling of straws and records } & 176\end{array}$

\section{PART III. QUALITY ASSURANCE}

\begin{tabular}{rlr}
\hline Chapter 7 & Quality assurance and quality control & 179 \\
\hline 7.1 & Controlling for quality in the andrology laboratory & 179 \\
7.2 & The nature of errors in semen analysis & 179 \\
7.3 & Minimizing statistical sampling error & 180 \\
7.4 & The quality assurance programme & 182 \\
7.5 & Laboratory procedures manual & 182 \\
7.6 & Internal quality control & 182 \\
7.6 .1 & Purchased QC samples & 183 \\
7.6 .2 & Laboratory-made QC samples & 183 \\
7.6 .3 & Stored samples (purchased or laboratory-made) & 183 \\
7.6 .4 & Fresh QC samples (laboratory-made) & 184 \\
7.7 & Statistical procedures for analysing and reporting within- and & 185 \\
& among-tec hnician systematic errors & 185 \\
7.7 .1 & The X chart & 188 \\
7.7 .2 & The S chart & 189 \\
7.8 & QC for percentages & 189 \\
7.9 & Assessing X and S charts & 189 \\
7.9 .1 & How to recognize out-of-control values & 190 \\
7.9 .2 & Causes of out-of-control values & 191 \\
7.9 .3 & Responses to out-of-control values & \\
7.10 & Statistical procedures for analysing and reporting among-technician & 191 \\
& variability & 191 \\
7.10 .1 & Comparing results from two or more technicians & 194 \\
7.10 .2 & Monitoring monthly means & 194 \\
7.11 & External quality control and quality assurance & 196 \\
7.11 .1 & Assessment of EQC results & 197 \\
7.11 .2 & Responses to out-of-control results & 197 \\
7.12 & Frequency and priority of quality control &
\end{tabular}


7.13 Training 198

7.13.1 Practical hints when experiencing difficulty assessing sperm concentration 198

7.13.2 Practical hints when experiencing difficulty assessing sperm morphology 200

7.13.3 Practical hints when experiencing difficulty assessing sperm motility 200

7.13.4 Practical hints when experiencing difficulty assessing sperm vitality 202

\section{APPENDICES}

\begin{tabular}{lll} 
Appendix 1 & Reference values and semen nomenclature & 223 \\
\hline
\end{tabular}

$\begin{array}{llr}\text { A1.1 Reference values } & 223\end{array}$

$\begin{array}{lll}\text { A1.2 Nomenclature } & 225\end{array}$

\begin{tabular}{lr} 
Appendix 2 Equipment and safety & 227 \\
\hline
\end{tabular}

$\begin{array}{lll}\text { A2.1 } & \text { Basic supplies needed in an andrology laboratory } & 227\end{array}$

A2.2 Potential biohazards in an andrology laboratory 230

A2.3 Safety procedures for laboratory personnel 230

A2.4 Safety procedures for laboratory equipment 232

A2.5 Safety precautions when handling liquid nitrogen 233

Appendix 3 Microscopy $\quad 234$

$\begin{array}{llr}\text { A3.1 Loading the sample } & 234\end{array}$

$\begin{array}{ll}\text { A3.2 Adjusting the oculars } & 236\end{array}$

A3.3 Focusing the image 236

A3.4 Focusing the oculars 236

A3.5 Focusing the light condenser 236

A3.6 Centring the condenser 237

A3.7 Adjusting the phase rings 237

$\begin{array}{lll}\text { A3.8 Fluorescence microscopy } & 237\end{array}$

\begin{tabular}{ll} 
Appendix 4 Stock solutions & 238 \\
\hline
\end{tabular}

$\begin{array}{lll}\text { A4.1 } & \text { Biggers, Whitten and Whittingham } & 238\end{array}$

A4.2 Dulbecco's phosphate-buffered saline $\quad 238$

A4.3 Earle's medium $\quad 239$

A4.4 Ham's F-10 medium 239

A4.5 Hanks' balanced salt solution $\quad 240$

A4.6 Human tubal fluid 240

A4.7 Krebs-Ringer medium 240

A4.8 Tris-buffered saline $\quad 241$

A4.9 Tyrode's solution 241

A4.10 Papanicolaou stain 241

Appendix 5 Cervical mucus $\quad 245$

$\begin{array}{llr}\text { A5.1 Introduction } & 245\end{array}$

A5.2 Collection and preservation of cervical mucus 246

$\begin{array}{ll}\text { A5.3 Evaluation of cervical mucus } & 247\end{array}$

Appendix 6 Record forms for semen and cervical mucus analyses $\quad 251$

$\begin{array}{lll}\text { A6.1 Template for a semen analysis recording form } & 251\end{array}$

A6.2 Template for a cervical mucus recording form 253 
\begin{tabular}{llr} 
Appendix 7 & Sampling errors and quality control & 254 \\
\hline
\end{tabular}

A7.1 Errors in measurement of sperm concentration 254

A7.2 The importance of understanding sampling errors 256

A7.3 Errors in measurement of percentages 257

A7.4 Production of semen samples for quality control 260

A7.5 Preparation of a video-recording for internal quality control of analysis of

A7.6 Preparation of diluted semen for internal quality control of determination

A7.7 Preparation of slides for internal quality control of assessment of sperm

A7.8 Calibration of equipment 269

Appendix 8 National external quality control programmes for semen analysis $\quad 271$

\section{FIGURES}

Fig. 2.1 Variation in total number of spermatozoa and sperm concentration over a one-and-a-half-year period 9

Fig. 2.2 Non-specific aggregation of spermatozoa in semen 19

Fig. 2.3 Schematic diagram of different extents of sperm agglutination 20

Fig. 2.4 Aids to assessing sperm motility 24

Fig. 2.5 Eosin-nigrosin smear observed in brightfield optics 28

Fig. 2.6 Schematic representation of typical morphological changes of human
spermatozoa subjected to hypo-osmotic stress

Fig. 2.7 The improved Neubauer haemocytometer 34

Fig. 2.8 Which spermatozoa to count in the grid squares 36

Fig. 2.9 Scanning the entire coverslip for the presence of motile spermatozoa

Fig. 2.10 Morphologically "normal" spermatozoa $\quad 58$

Fig. 2.11 Semen smearing methods for sperm morphology 59

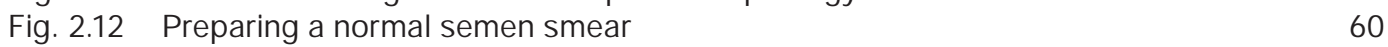

Fig. 2.13 Schematic drawings of some abnormal forms of human spermatozoa 70

Fig. 2.14 Peroxidase-positive and -negative cells in human semen $\quad 105$

$\begin{array}{ll}\text { Fig. } 3.1 \text { Leukocytes in semen } & 120\end{array}$

Fig. 3.2 The Kremer sperm penetration meter 128

Fig. 3.3 Standard terminology for variables measured by CASA systems 139

Fig. 4.1 Chemiluminescence generated in response to opsonized zymosan treatment $\quad 145$

Fig. 4.2 Relative contributions made by leukocyte and sperm subpopulations to the reactive-oxygen-generating capacity of the cell suspension $\quad 146$

Fig. 4.3 Fluorescent Pisum sativum agglutinin (PSA) staining of human spermatozoa 149

Fig. 4.4 Phase-contrast micrograph of a zona-free hamster oocyte containing $\begin{array}{ll}\text { human spermatozoa } & 157\end{array}$

$\begin{array}{lll}\text { Fig. 7.1 An } \mathrm{X}_{\text {bar }} \text { chart for sperm concentration } & 187\end{array}$

Fig. 7.2 An S chart for sperm concentration 189

$\begin{array}{ll}\text { Fig. 7.3 A Bland-Altman plot of manual and CASA estimates of percentage } & 192 \\ \text { progressive sperm motility }\end{array}$

Fig. 7.4 A Youden plot of estimates of the concentration of spermatozoa 193

Fig. A2.1 Nomogram for reading relative centrifugal force (RCF) from rotor radius
and rotation speed

Fig. A5.1 Examples of fern formation in cervical mucus air-dried on a glass slide 246

Fig. A7.1 The acceptable differences between two replicate counts as a function
of the total number of spermatozoa assessed 
Fig. A7.2 The acceptable differences between two replicate assessments of percentage as a function of the true percentage and the total number of spermatozoa assessed 258

Fig. A7.3 Aid to assessing sperm motility

Fig. A7.4 View through an ocular with reticle (red grid)

Fig. A7.5 View of the videotaped image of the stage micrometer on the monitor and the drawn overlay

\section{BOXES}

Box 2.1 Confirming the compatibility of semen collection vessels 11

Box 2.2 Preparation of bromelain $\quad 14$

Box 2.3 Thorough mixing of semen 18

Box 2.4 Depth of wet preparations 18

Box 2.5 Errors in estimating percentages 24

Box 2.6 Comparison of replicate percentages 25

Box 2.7 Errors in estimating numbers 36

Box 2.8 Achieving 200 spermatozoa per replicate in the central three grids of
the improved Neubauer chamber

Box 2.9 Volume observed per high-power field of a $20-\mu \mathrm{m}$-deep wet preparation 38

Box 2.10 Comparison of replicate counts 42

Box 2.11 Achieving 200 spermatozoa per replicate in all nine grids of the improved Neubauer chamber 48

Box 2.12 Achieving 200 spermatozoa per replicate in a 100- $\mu \mathrm{m}$-deep, large-volume disposable chamber 52

Box 2.13 Volume observed per high-power field in a 100- $\mu \mathrm{m}$-deep, large-volume
disposable chamber

Box 2.14 Mounting media 63

Box 3.1 Preparation of a wax-petroleum jelly mixture 123

Box 3.2 Volume observed per high-power field in a 100- $\mu \mathrm{m}$-deep mucus preparation $\quad 124$

Box 4.1 Induction of ovulation in hamsters $\quad 154$

Box 4.2 Preparation of glass pipettes $\quad 155$

Box 6.1 Reasons for cryopreservation of spermatozoa $\quad 170$

Box 6.2 Risk assessment of cryopreservation and storage of human semen $\quad 171$

Box 7.1 Terminology in quality assurance and quality control 180

Box 7.2 Determining the values for the warning and action control limits of an $\mathrm{X}_{\mathrm{bar}}$ chart $\quad 186$

Box 7.3 Alternative method for calculating the $X_{b a r}$ control limits from the pooled $\begin{array}{ll}\text { standard deviation } & 187\end{array}$

Box 7.4 Determining the values for the warning and action control limits of an S chart 188

Box 7.5 Basic control rules for QC charts 190

Box 7.6 Assessing systematic differences among technicians 195

Box 7.7 Main features of IQC procedures 196

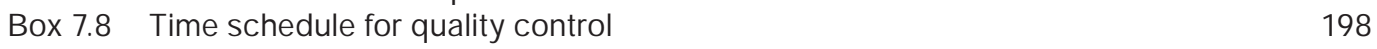

Box 7.9 Summary of QC tests 198

$\begin{array}{lll}\text { Box A2.1 Calculating centrifugal forces } & 230\end{array}$

Box A3.1 The objective lens $\quad 235$

Box A5.1 Determining the volume of mucus collected 247

Box A5.2 Volume observed per high-power field in a 100- $\mu \mathrm{m}$-deep mucus preparation 249 
TABLES

Table 2.1 Acceptable differences between two percentages for a given average, determined from replicate counts of 200 spermatozoa (total 400 counted)

Table 2.2 Rounded sampling errors (\%) according to total number of spermatozoa counted

Table 2.3 Semen dilutions required, how to make them, which chambers to use and potential areas to assess

Table 2.4 Acceptable differences between two replicate counts for a given sum

Table 2.5 Acceptable differences between two counts for a given sum: low concentrations 50

Table 2.6 Explanations used in commentaries to the morphological plates (1-14) 71

Table 2.7 How much semen to use for an immunobead test 111

Table 3.1 Calculation of indices of multiple sperm defects 116

Table 3.2 Sperm defect indices for men from fertile and infertile couples 117

$\begin{array}{lll}\text { Table 3.3 Rank order of sperm penetration density } & 129\end{array}$

$\begin{array}{lll}\text { Table } 3.4 & \text { Classification of the capillary tube test results } & 129\end{array}$

Table 7.1 Factors for determining control limits for $\mathrm{X}_{\mathrm{bar}}$ charts and S charts based on
the average standard deviation $\left(\mathrm{S}_{\mathrm{bar}}\right)$

Table 7.2 Sources of variation (error) in assessing sperm concentration and proposed

Table 7.3 Sources of variation (error) in assessing sperm morphology and proposed solutions

Table 7.4 Sources of variation (error) in assessing sperm motility and proposed solutions

Table 7.5 Sources of variation (error) in assessing sperm vitality and proposed solutions

201

Table A1.1 Lower reference limits (5th centiles and their $95 \%$ confidence intervals) for semen characteristics

Table A1.2 Distribution of values for semen parameters from men whose partners became pregnant within 12 months of discontinuing contraceptive use 225

Table A1.3 Nomenclature related to semen quality 226

Table A7.1 Acceptable differences between two replicate counts for a given sum 255

Table A7.2 Acceptable differences between two percentages for a given average, determined from replicate counts of 100 spermatozoa (total 200 counted) 259

Table A7.3 Acceptable differences between two percentages for a given average, determined from replicate counts of 200 spermatozoa (total 400 counted) 259

Table A7.4 Acceptable differences between two percentages for a given average, determined from replicate counts of 400 spermatozoa (total 800 counted) 


\section{Acknowledgements}

This publication was produced by the UNDP/UNFPA/WHO/World Bank Special Programme of Research, Development and Research Training in Human Reproduction (HRP), WHO Department of Reproductive Health and Research (RHR). The participation of the following individuals in the preparation and editing of this manual is gratefully acknowledged:

\section{Editor-in-chief}

\section{Dr Trevor G Cooper}

Centre of Reproductive Medicine and Andrology of the University,

Münster, Germany

(WHO Collaborating Centre

for Research in Male Reproduction)

\section{Editorial team}

\section{Dr J ohn Aitken}

Biological Sciences

School of Life and Environmental Sciences

University Drive

Callaghan, New South Wales, Australia

\section{DrJ acques Auger}

Service de Biologie de la Réproduction

Pavillon Cassini Hôpital Cochin

Paris, France

\section{Dr HW Gordon Baker}

University of Melbourne

Department of Obstetrics and

Gynaecology

Royal Women's Hospital

Carlton, Victoria, Australia

\section{Dr Chris LR Barratt}

Division of Maternal and

Child Health Sciences

The Medical School

Ninewells Hospital

Dundee, Scotland

\section{Dr Hermann M Behre}

Centre for Reproductive

Medicine and Andrology

Martin-Luther-University

Halle, Germany
Dr Lars Björndahl

Andrology Centre,

Karolinska University Hospital

and Institute,

Stockholm, Sweden

Ms Charlene Brazil

Center for Health and

the Environment

University of California

Davis, CA, USA

\section{Dr Christopher De J onge}

University of Minnesota

Reproductive Medicine Center

Minneapolis, MN, USA

\section{Dr Gustavo F Doncel}

CONRAD

Department of Obstetrics and

Gynecology Eastern Virginia

Medical School

Norfolk, VA, USA

\section{Dr Daniel Franken}

Department of Obstetrics and

Gynaecology

Tygerberg Hospital

Tygerberg, South Africa

\section{DrTrine B Haugen}

Faculty of Health Sciences

Oslo University College

Oslo, Norway

\section{Dr Aucky Hinting}

Andrology Unit, Department of Biomedicine School of Medicine

Airlangga University, Surabaya, Indonesia 
Mr Godwin E Imade

Department of Obstetrics and Gynaecology

Faculty of Medical Sciences University of J os

J os, Nigeria

\section{Dr Thinus F Kruger}

Reproductive Biology Unit

Stellenbosch University

Tygerberg, South Africa

Dr Hesbon 0 Odongo

Department of Zoology

University of Nairobi

Nairobi, Kenya

\section{Ms Elizabeth Noonan}

Fred Hutchinson Cancer Research Center

Statistical Center for HIV/AIDS Research and

Prevention

Seattle, WA, USA

\section{Dr Steven M Schrader}

National Institute for Occupational

Safety and Health

Centers for Disease Control and Prevention

Cincinnati, $\mathrm{OH}$, USA
Dr Christina CL Wang

Harbor-UCLA Medical Center

Torrance, CA, USA

\section{Dr William Shu-B iu Yeung}

Department of Obstetrics and Gynaecology

University of Hong Kong

Hong Kong SAR, China

WHO Secretariat, Department of Reproductive Health and Research

Dr Kirsten M Vogelsong

Scientist

Research Area Manager

Dr Sigrid von Eckardstein

Former Acting Research Area Manager

\section{Dr Michael T Mbizvo}

Director ad interim

\section{Ms Maud Keizer}

Secretary

Additional thanks go to: Ms Cathy Treece, Ms Charlene Tollner and Professor J im Overstreet (University of California, Davis, CA, USA) for producing the morphology micrographs and checking the media; Dr Rune Eliasson (Sophiahemmet Hospital, Stockholm, Sweden) for help in defining non-sperm cells; Dr Timothy Farley (World Health Organization, Geneva, Switzerland) for review of the sections on quality control; and Dr Gary N Clarke (The Royal Women's Hospital, Carlton, Australia), Dr Roelof Menkveld (Tygerberg Academic Hospital and University of Stellenbosch, Tygerberg, South Africa), and Professor P ieter Wranz (University of Stellenbosch, Tygerberg, South Africa) for providing additional information used in the compilation of the manual.

The financial support of the International Society of Andrology is gratefully acknowledged.

This edition of the manual is dedicated to the memory of the late Geoffrey Waites (1928-2005), former manager of the WHO Task Force on Methods for the Regulation of Male Fertility and coeditor of the second, third and fourth editions of this laboratory manual. The editorial committee's devotion to its task was driven by its appreciation of Geoff's honesty, fairness and concern for the underprivileged. 


\section{Acronyms and abbreviations used in this manual}

$\begin{array}{ll}\text { Ab } & \text { antibody } \\ \text { AI } & \text { artificial insemination } \\ \text { AID } & \text { artificial insemination with donor semen } \\ \text { AIH } & \text { artificial insemination with husband's semen } \\ \text { ALH } & \text { amplitude of lateral head displacement } \\ \text { ANOVA } & \text { analysis of variance } \\ \text { APAAP } & \text { alkaline phosphatase-anti-alkaline phosphatase complex } \\ \text { AR } & \text { acrosome-reacted } \\ \text { ART } & \text { assisted reproductive technology } \\ \text { ASA } & \text { anti-sperm antibody } \\ \text { BAEE } & \text { N-benzoyl-L-arginine ethyl ester } \\ \text { BCF } & \text { beat-cross frequency (Hz) } \\ \text { BSA } & \text { bovine serum albumin } \\ \text { BWW } & \text { Biggers, Whitten and Whittingham } \\ \text { CASA } & \text { computer-aided sperm analysis } \\ \text { CASMA } & \text { computer-aided sperm morphometric assessment } \\ \text { CBAVD } & \text { congenital bilateral absence of the vas deferens } \\ \text { CD } & \text { compact disk } \\ \text { CD } & \text { cytoplasmic droplet } \\ \text { CD45 } & \text { cluster of determination 45 (pan-leukocyte marker) } \\ \text { CD46 } & \text { cluster of determination 46 (acrosomal antigen) } \\ \text { CI } & \text { confidence interval } \\ \text { CL } & \text { confidence limits } \\ \text { CO } & \text { carbon dioxide } \\ \text { DMSO } & \text { dimethyl sulfoxide } \\ \text { DNA } & \text { deoxyribonucleic acid } \\ \text { DPBS } & \text { Dulbecco's phosphate-buffered saline } \\ \text { DVD } & \text { digital versatile disc } \\ \text { EDTA } & \text { ethylenediamine tetra-acetic acid } \\ \text { EQA } & \text { external quality assurance } \\ \text { EQC } & \text { external quality control } \\ \text { ERC } & \text { excess residual cytoplasm } \\ \text { FITC } & \text { fluorescein isothiocyanate } \\ \text { FMLP } & \text { formyl-methionyl-leucyl-phenylalanine } \\ \text { GIFT } & \text { gamete intrafallopian transfer } \\ \text { GPC } & \text { glycerophosphocholine } \\ \text { H2O } & \text { hydrogen peroxide } \\ \text { HBSS } & \text { Hanks' balanced salt solution } \\ \text { HBV } & \text { hepatitis B virus } \\ \text { hCG } & \text { human chorionic gonadotrophin } \\ \text { HCV } & \text { hepatitis C virus } \\ \text { HIV } & \text { human immunodeficiency virus } \\ \text { HOP } & \text { hamster oocyte penetration } \\ \text { HOS } & \text { hypo-osmotic swelling } \\ \text { HPF } & \text { high-power field } \\ \text { HRP } & \text { horseradish peroxidase } \\ & \end{array}$




\begin{tabular}{|c|c|}
\hline HSA & human serum albumin \\
\hline HTF & human tubal fluid \\
\hline IB & immunobead \\
\hline IBT & immunobead test \\
\hline ICSI & intracytoplasmic sperm injection \\
\hline $\lg$ & immunoglobulin \\
\hline IM & immotility \\
\hline IQC & internal quality control \\
\hline IU & international unit \\
\hline IUI & intrauterine insemination \\
\hline IVF & in-vitro fertilization \\
\hline KRM & Krebs-Ringer Medium \\
\hline LIN & linearity \\
\hline LLQ & lower limit of quantification \\
\hline LPF & low-power field \\
\hline MAD & mean angular displacement \\
\hline MAI & multiple anomalies index \\
\hline MAR & mixed antiglobulin reaction \\
\hline NA & numerical aperture \\
\hline NP & non-progressive (motility) \\
\hline PBS & phosphate-buffered saline \\
\hline PDCA & plan, do, check, act \\
\hline PMA & phorbol 12-myristate 13 -acetate \\
\hline PMSG & pregnant mare serum gonadotrophin \\
\hline PNPG & p-nitrophenol glucopyranoside \\
\hline PR & progressive (motility) \\
\hline PSA & $P$ isum sativum agglutinin \\
\hline QA & quality assurance \\
\hline QC & quality control \\
\hline RCF & relative centrifugal force \\
\hline RI & refractive index \\
\hline RNA & ribonucleic acid \\
\hline ROS & reactive oxygen species \\
\hline r.p.m. & revolutions per minute \\
\hline SD & standard deviation \\
\hline SDI & sperm deformity index \\
\hline SDS & sodium dodecyl sulfate \\
\hline SE & standard error \\
\hline SOP & standard operating procedure \\
\hline STR & straightness (VSL/VAP) \\
\hline TBS & Tris-buffered saline \\
\hline TGG & Tyrode's glucose glycerol \\
\hline TZI & teratozo ospermia index \\
\hline VAP & average path velocity \\
\hline VCL & curvilinear velocity \\
\hline VSL & straight-line (rectilinear) velocity \\
\hline WHO & World Health Organization \\
\hline WOB & wobble (VAP/VCL) \\
\hline
\end{tabular}




\section{CHAPTER 1 Background}

\subsection{Introduction}

The WHO laboratory manual for the examination of human semen and spermcervical mucus interaction was first published in 1980, in response to a growing need for the standardization of procedures for the examination of human semen. It has since been updated three times, and translated into a number of languages. Over the past 30 years, the manual has been recognized as providing global standards and has been used extensively by research and clinical laboratories throughout the world.

Despite this success, it has become apparent that some recommendations from previous editions of the manual needed to be revised in light of new evidence, and that some concepts needed more explanation and supporting evidence. Prompted by these considerations, WHO established an editorial committee to review all the methods described in the manual, with a view to endorsing, changing or updating them. In many instances, this proved difficult, as insufficient data had been obtained using the methods described in the manual. In some cases, single wellaccredited laboratories were obtaining consistent results, but these had not been confirmed by others. For these situations, the editorial committee developed a consensus position after evaluating the pertinent literature.

Additional recommendations were received from technicians and scientists, notably regarding the need for more detail for many of the methods described. Lack of detail in previous editions has meant that some laboratories have preferred to use methods described elsewhere, or have developed their own versions of methods, while still claiming to perform semen analysis according to the WHO manual. In order to make global comparisons easier, this edition of the manual therefore includes much greater detail, and the rationale is explained when alternative methods of analysis are presented. It is recommended that, when reporting results in published articles, laboratories should indicate which specific method was used when they refer to this manual.

\subsection{The fifth edition}

The fifth edition comprises three parts: semen analysis (Chapters 2-4), sperm preparation (Chapters 5 and 6 ) and quality assurance (Chapter 7). Part I, dealing with semen analysis, resembles that in previous editions, but is divided into three chapters: standard methods, which are robust routine procedures for determining semen quality; optional tests, which may be used in certain situations or by choice of the laboratory; and research tests, which are not currently regarded as routine. As semen culture is not normally performed in an andrology laboratory, this is mentioned only in the section on sterile collection of semen. The section on sperm preparation extends beyond the ejaculate to include spermatozoa obtained from the testis and epididymis. Interspersed with bulleted methodological instructions are Notes (explanations of methodology), Comments (interpretation of results) and Boxes (containing additional explanatory material). 
The main features of this fifth edition are outlined below.

- The chapters on semen analysis include details of all working solutions, procedures, calculations and interpretation, so that any given methodology is essentially complete, with minimal cross-referencing to other parts of the manual.

- The section on sperm preparation has been expanded, and a chapter on cryopreservation of spermatozoa has been added. Procedures related to the analysis of cervical mucus have been divided between the chapter on optional procedures and an appendix on characteristics of mucus.

- There are fewer appendices than in earlier editions, and they are restricted to specialized or only rarely needed information.

- Assessment of sperm numbers. The semen dilutions and the areas of the counting chamber used to assess the number of spermatozoa in a semen sample have been changed to allow 200 spermatozoa per replicate to be counted. The importance of sampling errors, and the certainty of the numerical results obtained, is emphasized. The editorial committee considered that total sperm number per ejaculate provides a more accurate assessment of testicular function than does sperm concentration, but for this semen volume has to be measured accurately.

- Assessment of azoospermia. Although superficially simple, the diagnosis of azoospermia is confounded by many factors, including large errors associated with counting few spermatozoa, the large number of microscopic fields to be analysed and difficulties in examining debris-laden sperm pellets.

Recommended changes include examining fixed, uncentrifuged samples and indicating the sensitivity of the counting methods employed. However, centrifugation methods necessary for accumulating sufficient numbers of cells for therapeutic procedures, and methods for the detection of motile spermatozoa in unfixed samples for assessment of post-vasectomy semen, are also included.

- Assessment of sperm motility. A major change from previous editions is in the categorization of sperm motility. It is now recommended that spermatozoa should be categorized as progressively motile, non-progressively motile and immotile (instead of grades $a, b, c$ or d).

- Assessment of sperm morphology. Some laboratories assess only normal forms, while others consider the type, location and extent of abnormality to be more important. Whether these or differential or semiquantitative assessments increase the value of semen analysis remains contentious. Evidence supporting the relationship between the percentage of normal forms (as defined by strict categorization or computer-aided assessment of morphology) and fertilization rates in vivo justifies trying to determine a morphologically distinct subpopulation of spermatozoa within semen. In this edition, more and better-quality micrographs of spermatozoa considered normal and borderline are included, accompanied by explanations of why each spermatozoon has been classified the way it has. This should help in training technicians to categorize spermatozoa consistently. Recent data from a fertile population have allowed reference values for the percentage of morphologically normal forms to be given. 
- Quality control. This chapter has been completely rewritten. Rigorous quality assurance for semen analysis is necessary for analytical methods to be robust. Hints and suggestions are given on how to improve laboratory performance when quality control results are unsatisfactory.

- Reference ranges and reference limits. Data characterizing the semen quality of fertile men, whose partners had a time to pregnancy of 12 months or less, provided the reference ranges for this manual. Raw data from between about 400 and 1900 semen samples, from recent fathers in eight countries on three continents, were used to generate the reference ranges. Conventional statistical tradition is to take the 2.5 th centile from a two-sided reference interval as the threshold below which values may be considered to come from a different population. However, a one-sided reference interval was considered to be more appropriate for semen, since high values of any parameter are unlikely to be detrimental to fertility. The 5 th centile is given as the lower reference limit, and the complete distribution for each semen parameter is also given in Appendix 1.

\subsection{Scope of the manual}

The methods described here are intended as guidelines to improve the quality of semen analysis and comparability of results. They should not necessarily be taken as obligatory by local, national or global laboratory accreditation bodies. Semen analysis may be useful in both clinical and research settings, for investigating male fertility status as well as monitoring spermatogenesis during and following male fertility regulation. 


\section{PART I. \\ Semen analysis}





\section{CHAPTER 2 Standard procedures}

\subsection{Introduction}

During ejaculation, semen is produced from a concentrated suspension of spermatozoa, stored in the paired epididymides, mixed with, and diluted by, fluid secretions from the accessory sex organs. It is emitted in several boluses. Comparison of pre- and post-vasectomy semen volumes reveals that about $90 \%$ of semen volume is made up of secretions from the accessory organs (Weiske, 1994), mainly the prostate and seminal vesicles, with minor contributions from the bulbourethral (Cowper's) glands and epididymides.

Semen has two major quantifiable attributes:

- the total number of spermatozoa: this reflects sperm production by the testes and the patency of the post-testicular duct system;

- the total fluid volume contributed by the various accessory glands: this reflects the secretory activity of the glands.

The nature of the spermatozoa (their vitality, motility and morphology) and the composition of seminal fluid are also important for sperm function.

During sexual intercourse, the initial, sperm-rich prostatic fraction of the ejaculated semen may come into contact with cervical mucus extending into the vagina (Sobrero \& MacLeod, 1962), with the rest of the fluid remaining as a pool in the vagina. In contrast, in the laboratory setting, the entire ejaculate is collected in one container, where spermatozoa are trapped in a coagulum developed from proteins of seminal vesicular origin. This coagulum is subsequently liquefied by the action of prostatic proteases, during which time its osmolality rises (Björndahl \& Kvist, 2003; Cooper et al., 2005).

There is some evidence that the quality of semen specimens varies depending on how the ejaculate is produced. Ejaculates produced by masturbation and collected into containers in a room near the laboratory can be of lower quality than those recovered from non-spermicidal condoms used during intercourse at home (Zavos \& Goodpasture, 1989). This difference may reflect a different form of sexual arousal, since the time spent producing a sample by masturbation-reflecting the extent of seminal emission before ejaculation-also influences semen quality (Pound et al., 2002).

Under given conditions of collection, semen quality depends on factors that usually cannot be modified, such as sperm production by the testes, accessory organ secretions and recent (particularly febrile) illness, as well as other factors, such as abstention time, that should be recorded and taken into account in interpreting the results.

The results of laboratory measurements of semen quality will depend on:

- Whether a complete sample is collected. During ejaculation the first semen fractions voided are mainly sperm-rich prostatic fluids, whereas later fractions are dominated by seminal vesicular fluid (Björndahl \& Kvist, 2003). Therefore, 
losing the first (sperm-rich) portion of the ejaculate has more influence on the results of semen analysis than does losing the last portion.

- The activity of the accessory sex glands, the fluids of which dilute the concentrated epididymal spermatozoa at ejaculation (Eliasson, 2003). Sperm concentration is not a direct measure of testicular sperm output, as it is influenced by the functioning of other reproductive organs; however, the total number of sperm ejaculated (sperm concentration multiplied by semen volume) is. For example, sperm concentrations in semen from young and old men may be the same, but total sperm numbers may differ, as both the volume of seminal fluid and total sperm output decrease with age, at least in some populations ( $\mathrm{Ng}$ et al., 2004).

- The time since the last sexual activity. In the absence of ejaculation, spermatozoa accumulate in the epididymides, then overflow into the urethra and are flushed out in urine (Cooper et al., 1993; De J onge et al., 2004). Sperm vitality and chromatin are unaffected by increased length of abstinence (Tyler et al., 1982b; De J onge et al., 2004) unless epididymal function is disturbed (CorreaPerez et al., 2004).

- The penultimate abstinence period. As the epididymides are not completely emptied by one ejaculation (Cooper et al., 1993), some spermatozoa remain from the time of the previous ejaculation. This influences the range of age and quality of spermatozoa in the ejaculate (Tyler et al., 1982a). The extent of this influence is difficult to ascertain and it is rarely taken into account.

- The size of the testis, which influences the total number of spermatozoa per ejaculate (Handelsman et al., 1984; WHO, 1987; Behre et al., 2000; Andersen et al., 2000). Testicular size reflects the level of spermatogenic activity, which also affects sperm morphology (Holstein et al., 2003).

Comment: The large biological variation in semen quality (Castilla et al., 2006) reflects the many factors listed above, and requires that all measurements on semen be precise.

These variable, and largely uncontrollable, factors explain the well-known intraindividual variation in semen composition (Baker \& Kovacs, 1985; Alvarez et al., 2003). Fig. 2.1 shows the variations over time in semen composition, as assessed by WHO-recommended methods, of five healthy young volunteers participating in the placebo arm of a male hormonal contraception study. Such variablility has consequences for the interpretation of semen analyses:

- It is impossible to characterize a man's semen quality from evaluation of a single semen sample.

- It is helpful to examine two or three samples to obtain baseline data (Poland et al., 1985; Berman et al., 1996; Carlsen et al., 2004; Castilla et al., 2006; Keel, 2006).

While measurements made on the whole population of ejaculated spermatozoa cannot define the fertilizing capacity of the few that reach the site of fertilization, semen analysis nevertheless provides essential information on the clinical status 
of an individual. All aspects of semen collection and analysis must be done by properly standardized procedures if the results are to provide valid, useful information. The tests described in this chapter are accepted procedures that constitute the essential steps in semen evaluation.

Fig. 2.1 Variation in total number of spermatozoa and sperm concentration over a one-and-a-half-year period
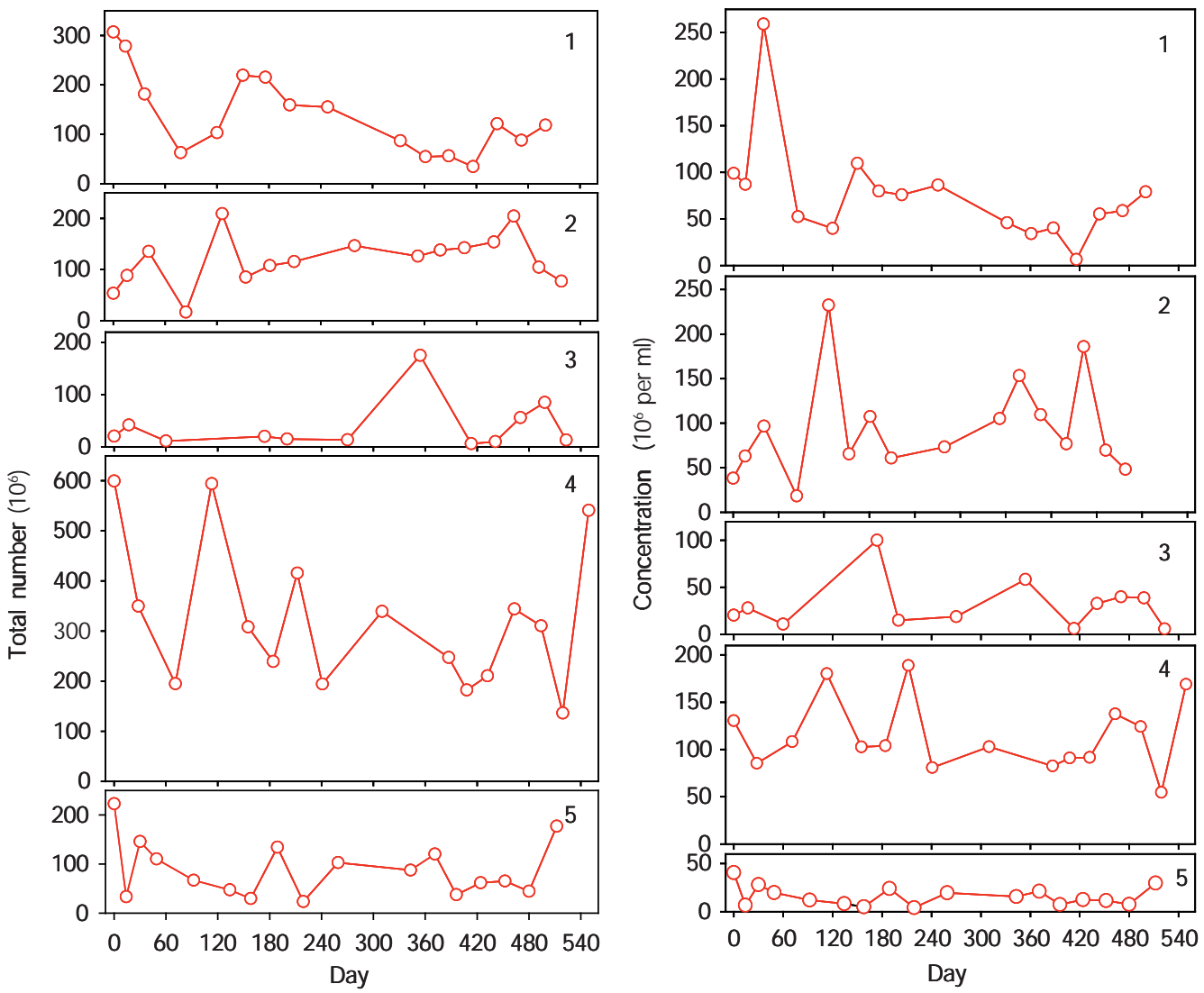

Data courtesy of Schering Plough and Bayer Schering Pharma AG.

Semen analysis involves the following steps (which are described in detail in subsequent sections).

In the first 5 minutes:

- Placing the specimen container on the bench or in an incubator $\left(37^{\circ} \mathrm{C}\right)$ for liquefaction.

Between 30 and 60 minutes:

- Assessing liquefaction and appearance of the semen.

- Measuring semen volume. 
- Measuring semen pH (if required).

- Preparing a wet preparation for assessing microscopic appearance, sperm motility and the dilution required for assessing sperm number.

- Assessing sperm vitality (if the percentage of motile cells is low).

- Making semen smears for assessing sperm morphology.

- Making semen dilutions for assessing sperm concentration.

- Assessing sperm number.

- Performing the mixed antiglobulin reaction (MAR) test (if required).

- Assessing peroxidase-positive cells (if round cells are present).

- Preparing spermatozoa for the immunobead test (if required).

- Centrifuging semen (if biochemical markers are to be assayed).

Within 3 hours:

- Sending samples to the microbiology laboratory (if required).

After 4 hours:

- Fixing, staining and assessing smears for sperm morphology.

Later on the same day (or on a subsequent day if samples are frozen):

- Assaying accessory gland markers (if required).

- Performing the indirect immunobead test (if required).

\subsection{Sample collection}

\subsubsection{Preparation}

- The sample should be collected in a private room near the laboratory, in order to limit the exposure of the semen to fluctuations in temperature and to control the time between collection and analysis (see Sections 2.2.5 and 2.2.6 for exceptions).

- The sample should be collected after a minimum of 2 days and a maximum of 7 days of sexual abstinence. If additional samples are required, the number of days of sexual abstinence should be as constant as possible at each visit.

- The man should be given clear written and spoken instructions concerning the collection of the semen sample. These should emphasize that the semen sample needs to be complete and that the man should report any loss of any fraction of the sample.

- The following information should be recorded on the report form (see Appendix 6, section A6.1): the man's name, birth date and personal code number, the period of abstinence, the date and time of collection, the completeness of the sample, any difficulties in producing the sample, and the interval between collection and the start of the semen analysis. 
2.2.2 Collection of semen for diagnostic or research purposes

- The sample should be obtained by masturbation and ejaculated into a clean, wide-mouthed container made of glass or plastic, from a batch that has been confirmed to be non-toxic for spermatozoa (see Box 2.1).

- The specimen container should be kept at ambient temperature, between $20^{\circ} \mathrm{C}$ and $37^{\circ} \mathrm{C}$, to avoid large changes in temperature that may affect the spermatozoa after they are ejaculated into it. It must be labelled with the man's name and identification number, and the date and time of collection.

- The specimen container is placed on the bench or in an incubator $\left(37^{\circ} \mathrm{C}\right)$ while the semen liquefies.

- Note in the report if the sample is incomplete, especially if the first, sperm-rich fraction may be missing. If the sample is incomplete, a second sample should be collected, again after an abstinence period of 2-7 days.

\section{B ox 2,1 Confirming the compatibility of semen collection vessels}

Select several semen samples with high sperm concentration and good sperm motility. Place half of each specimen in a container known to be non-toxic (control) and the other half in the container being tested. Assess sperm motility (see Section 2.5) at hourly intervals in replicate at room temperature or at $37^{\circ} \mathrm{C}$ for 4 hours. If there are no differences at each time point between control and test assessments ( $P>0.05$ as judged by a paired $t$-test), the test containers can be considered to be non-toxic to spermatozoa and to meet semen collection requirements.

\subsubsection{Sterile collection of semen for assisted reproduction}

This is performed as for diagnostic collection (see Section 2.2.2) but the specimen containers, pipette tips and pipettes for mixing must be sterile.

\subsubsection{Sterile collection of semen for microbiological analysis}

In this situation, microbiological contamination from non-semen sources (e.g. commensal organisms from the skin) must be avoided. The specimen containers, pipette tips and pipettes for mixing must be sterile.

The man should:

- Pass urine.

- Wash hands and penis with soap, to reduce the risk of contamination of the specimen with commensal organisms from the skin.

- Rinse away the soap.

- Dry hands and penis with a fresh disposable towel.

- Ejaculate into a sterile container.

Note: The time between collection of the semen sample and the start of the investigation by the microbiological laboratory should not exceed 3 hours. 


\subsubsection{Collection of semen at home}

- A sample may be collected at home in exceptional circumstances, such as a demonstrated inability to produce a sample by masturbation in the clinic or the lack of adequate facilities near the laboratory.

- The man should be given clear written and spoken instructions concerning the collection and transport of the semen sample. These should emphasize that the semen sample needs to be complete, i.e. all the ejaculate is collected, including the first, sperm-rich portion, and that the man should report any loss of any fraction of the sample. It should be noted in the report if the sample is incomplete.

- The man should be given a pre-weighed container, labelled with his name and identification number.

- The man should record the time of semen production and deliver the sample to the laboratory within 1 hour of collection.

- During transport to the laboratory, the sample should be kept between $20{ }^{\circ} \mathrm{C}$ and $37^{\circ} \mathrm{C}$.

- The report should note that the sample was collected at home or another location outside the laboratory.

\subsubsection{Collection of semen by condom}

- A sample may be collected in a condom during sexual intercourse only in exceptional circumstances, such as a demonstrated inability to produce a sample by masturbation.

- Only special non-toxic condoms designed for semen collection should be used; such condoms are available commercially.

- The man should be given information from the manufacturer on how to use the condom, close it, and send or transport it to the laboratory.

- The man should record the time of semen production and deliver the sample to the laboratory within 1 hour of collection.

- During transport to the laboratory, the sample should be kept between $20{ }^{\circ} \mathrm{C}$ and $37^{\circ} \mathrm{C}$.

- The report should note that the sample was collected by means of a special condom during sexual intercourse at home or another location outside the laboratory.

Note: Ordinary latex condoms must not be used for semen collection because they contain agents that interfere with the motility of spermatozoa (J ones et al., 1986).

Comment 1: Coitus interruptus is not a reliable means of semen collection, because the first portion of the ejaculate, which contains the highest number of spermatozoa, may be lost. Moreover, there may be cellular and bacteriological contamination of the sample, and the low $\mathrm{pH}$ of the vaginal fluid could adversely affect sperm motility. 
Comment 2: If a man cannot provide a semen sample, the postcoital test (see Section 3.3.1) may provide some information about his spermatozoa.

\subsubsection{Safe handling of specimens}

Semen samples may contain dangerous infectious agents (e.g. human immunodeficiency virus (HIV), hepatitis viruses or herpes simplex virus) and should therefore be handled as a biohazard. If the sample is to be processed for bioassay, intra- uterine insemination (IUI), in-vitro fertilization (IVF) or intracytoplasmic sperm injection (ICSI) (see Section 5.1), or if semen culture is to be performed (see Section 2.2.4), sterile materials and techniques must be used. Safety guidelines as outlined in Appendix 2 should be strictly followed; good laboratory practice is fundamental to laboratory safety (WHO, 2004).

\subsection{Initial macroscopic examination}

Semen analysis should begin with a simple inspection soon after liquefaction, preferably at 30 minutes, but no longer than 1 hour after ejaculation, to prevent dehydration or changes in temperature from affecting semen quality.

\subsubsection{Liquefaction}

Immediately after ejaculation into the collection vessel, semen is typically a semisolid coagulated mass. Within a few minutes at room temperature, the semen usually begins to liquefy (become thinner), at which time a heterogeneous mixture of lumps will be seen in the fluid. As liquefaction continues, the semen becomes more homogeneous and quite watery, and in the final stages only small areas of coagulation remain. The complete sample usually liquefies within 15 minutes at room temperature, although rarely it may take up to 60 minutes or more. If complete liquefaction does not occur within 60 minutes, this should be recorded. Semen samples collected at home or by condom will normally have liquefied by the time they arrive in the laboratory.

Normal liquefied semen samples may contain jelly-like granules (gelatinous bodies) which do not liquefy; these do not appear to have any clinical significance. The presence of mucus strands, however, may interfere with semen analysis.

Note 1: Liquefaction can be recognized both macroscopically, as described above, and microscopically. Immobilized spermatozoa gain the ability to move as the semen liquefies. If immobilized spermatozoa are observed on microscopic examination, more time must be allowed for the liquefaction process to be completed.

Note 2: During liquefaction, continuous gentle mixing or rotation of the sample container on a two-dimensional shaker, either at room temperature or in an incubator set at $37^{\circ} \mathrm{C}$, can help to produce a homogeneous sample.

Note 3: If the semen does not liquefy within 30 minutes, do not proceed with semen analysis but wait for another 30 minutes. If liquefaction has not occurred within 60 minutes, proceed as in Section 2.3.1.1. 


\subsubsection{Delayed liquefaction}

Occasionally samples may not liquefy, making semen evaluation difficult. In these cases, additional treatment, mechanical mixing or enzymatic digestion may be necessary.

1. Some samples can be induced to liquefy by the addition of an equal volume of physiological medium (e.g. Dulbecco's phosphate-buffered saline; see Appendix 4, section A4.2), followed by repeated pipetting.

2. Inhomogeneity can be reduced by repeated (6-10 times) gentle passage through a blunt gauge 18 (internal diameter $0.84 \mathrm{~mm}$ ) or gauge 19 (internal diameter $0.69 \mathrm{~mm}$ ) needle attached to a syringe.

3. Digestion by bromelain, a broad-specificity proteolytic enzyme (EC 3.4.22.32), may help to promote liquefaction (see Box 2.2).

\section{Box 2.2 Preparation of bromelain}

Prepare $10 \mathrm{IU} / \mathrm{ml}$ bromelain in Dulbecco's phosphate-buffered saline (see Appendix 4, section A4.2); it is difficult to dissolve but, with mixing, most should dissolve within 15-20 minutes. Dilute semen $1+1(1: 2)$ with the $10 \mathrm{IU} / \mathrm{ml}$ bromelain, stir with a pipette tip, and incubate at $37^{\circ} \mathrm{C}$ for 10 minutes. Mix the sample well before further analysis.

Comment: These treatments may affect seminal plasma biochemistry, sperm motility and sperm morphology, and their use must be recorded. The $1+1(1: 2)$ dilution of semen with bromelain must be accounted for when calculating sperm concentration.

\subsubsection{Semen viscosity}

After liquefaction, the viscosity of the sample can be estimated by gently aspirating it into a wide-bore (approximately $1.5 \mathrm{~mm}$ diameter) plastic disposable pipette, allowing the semen to drop by gravity and observing the length of any thread. A normal sample leaves the pipette in small discrete drops. If viscosity is abnormal, the drop will form a thread more than $2 \mathrm{~cm}$ long.

Alternatively, the viscosity can be evaluated by introducing a glass rod into the sample and observing the length of the thread that forms upon withdrawal of the rod. The viscosity should be recorded as abnormal when the thread exceeds $2 \mathrm{~cm}$.

In contrast to a partially unliquefied sample, a viscous semen specimen exhibits homogeneous stickiness and its consistency will not change with time. High viscosity can be recognized by the elastic properties of the sample, which adheres strongly to itself when attempts are made to pipette it. The methods to reduce viscosity are the same as those for delayed liquefaction (see Section 2.3.1.1). 
Comment: High viscosity can interfere with determination of sperm motility, sperm concentration, detection of antibody-coated spermatozoa and measurement of biochemical markers.

\subsubsection{Appearance of the ejaculate}

A normal liquefied semen sample has a homogeneous, grey-opalescent appearance. It may appear less opaque if the sperm concentration is very low; the colour may also be different, i.e. red-brown when red blood cells are present (haemospermia), or yellow in a man with jaundice or taking certain vitamins or drugs.

\subsubsection{Semen volume}

The volume of the ejaculate is contributed mainly by the seminal vesicles and prostate gland, with a small amount from the bulbourethral glands and epididymides. Precise measurement of volume is essential in any evaluation of semen, because it allows the total number of spermatozoa and non-sperm cells in the ejaculate to be calculated.

The volume is best measured by weighing the sample in the vessel in which it is collected.

- Collect the sample in a pre-weighed, clean, disposable container.

- Weigh the vessel with semen in it.

- Subtract the weight of the container.

- Calculate the volume from the sample weight, assuming the density of semen to be $1 \mathrm{~g} / \mathrm{ml}$ (Auger et al., 1995). (Semen density varies between 1.043 and $1.102 \mathrm{~g} / \mathrm{ml}$ (Huggins et al., 1942; B razil et al., 2004a; Cooper et al., 2007).)

Note: Empty specimen containers may have different weights, so each container should be individually pre-weighed. The weight may be recorded on the container before it is given to the client. Use a permanent marker pen on the vessel itself or on a label. If a label is used for recording the weight, it should be attached before the empty container is weighed.

Alternatively, the volume can be measured directly.

- Collect the sample directly into a modifed graduated glass measuring cylinder with a wide mouth. These can be obtained commercially.

- Read the volume directly from the graduations ( $0.1 \mathrm{ml}$ accuracy).

Note: Measuring volume by aspirating the sample from the specimen container into a pipette or syringe, or decanting it into a measuring cylinder, is not recommended, because not all the sample will be retrieved and the volume will therefore be underestimated. The volume lost can be between 0.3 and $0.9 \mathrm{ml}$ (Brazil et al., 2004a; Iwamoto et al., 2006; Cooper et al., 2007). 
Comment 1: Low semen volume is characteristic of obstruction of the ejaculatory duct or congenital bilateral absence of the vas deferens (CBAVD) (de la Taille et al., 1998; Daudin et al., 2000; von Eckardstein et al., 2000; Weiske et al., 2000), a condition in which the seminal vesicles are also poorly developed.

Comment 2: Low semen volume can also be the result of collection problems (loss of a fraction of the ejaculate), partial retrograde ejaculation or androgen deficiency.

Comment 3: High semen volume may reflect active exudation in cases of active inflammation of the accessory organs.

\subsubsection{Lower reference limit}

The lower reference limit for semen volume is $1.5 \mathrm{ml}$ (5th centile, $95 \%$ confidence interval (CI) 1.4-1.7).

\subsubsection{Semen $\mathrm{pH}$}

The $\mathrm{pH}$ of semen reflects the balance between the $\mathrm{pH}$ values of the different accessory gland secretions, mainly the alkaline seminal vesicular secretion and the acidic prostatic secretion. The $\mathrm{pH}$ should be measured after liquefaction at a uniform time, preferably after 30 minutes, but in any case within 1 hour of ejaculation since it is influenced by the loss of $\mathrm{CO}_{2}$ that occurs after production.

For normal samples, pH paper in the range 6.0 to 10.0 should be used.

- Mix the semen sample well (see Box 2.3).

- Spread a drop of semen evenly onto the pH paper.

- Wait for the colour of the impregnated zone to become uniform ( $<30$ seconds).

- Compare the colour with the calibration strip to read the $\mathrm{pH}$.

Note: The accuracy of the $\mathrm{pH}$ paper should be checked against known standards.

For viscous samples, the $\mathrm{pH}$ of a small aliquot of the semen can be measured using a $\mathrm{pH}$ meter designed for measurement of viscous solutions (Haugen $\&$ Grotmol, 1998).

\subsubsection{Reference values}

There are currently few reference values for the $\mathrm{pH}$ of semen from fertile men. Pending more data, this manual retains the consensus value of 7.2 as a lower threshold value. 
Comment 1: If the $\mathrm{pH}$ is less than 7.0 in a semen sample with low volume and low sperm numbers, there may be ejaculatory duct obstruction or congenital bilateral absence of the vas deferens (de la Taille et al., 1998; Daudin et al., 2000; von Eckardstein et al., 2000; Weiske et al., 2000), a condition in which seminal vesicles are also poorly developed.

Comment 2: Semen pH increases with time, as natural buffering decreases, so high $\mathrm{pH}$ values may provide little clinically useful information.

\subsection{Initial microscopic investigation}

A phase-contrast microscope is recommended for all examinations of unstained preparations of fresh semen (see Appendix 3 for how to set up a microscope). An initial microscopic examination of the sample involves scanning the preparation at a total magnification of $\times 100$ (i.e. a combination of a $\times 10$ objective lens with a $\times 10$ ocular lens).

This provides an overview of the sample, to reveal:

- mucus strand formation;

- sperm aggregation or agglutination;

- the presence of cells other than spermatozoa, e.g. epithelial cells, "round cells" (leukocytes and immature germ cells) and isolated sperm heads or tails.

The preparation should then be observed at $\times 200$ or $\times 400$ total magnification (i.e. a combination of a $\times 20$ or a $\times 40$ objective with a $\times 10$ ocular). This permits:

- assessment of sperm motility (see Section 2.5);

- determination of the dilution required for accurate assessment of sperm number (see Section 2.8).

2.4.1 Thorough mixing and representative sampling of semen

The nature of the liquefied ejaculate makes taking a representative sample of semen for analysis problematical. If the sample is not well mixed, analysis of two separate aliquots may show marked differences in sperm motility, vitality, concentration and morphology. To be certain of obtaining reproducible data, the sample should be thoroughly mixed before aliquots are taken for assessment (see Box 2.3), and results for replicate aliquots should agree before the values are accepted. Agreement between replicates is determined for sperm numbers by the Poisson distribution (see Boxes 2.7 and 2.10, and Tables 2.4 and 2.5) and for percentages by the binomial distribution (see Boxes 2.5 and 2.6, and Table 2.1). 


\section{Box 2.3 Thorough mixing of semen}

Before removing an aliquot of semen for assessment, mix the sample well in the original container, but not so vigorously that air bubbles are created. This can be achieved by aspirating the sample 10 times into a wide-bore (approximately $1.5 \mathrm{~mm}$ diameter) disposable plastic pipette (sterile when necessary). Do not mix with a vortex mixer at high speed as this will damage spermatozoa.

\subsubsection{Making a wet preparation}

- Mix the semen sample well (see Box 2.3).

- Remove an aliquot of semen immediately after mixing, allowing no time for the spermatozoa to settle out of suspension.

- Remix the semen sample before removing replicate aliquots.

The volume of semen and the dimensions of the coverslip must be standardized, so that the analyses are carried out on a preparation of fixed depth of about $20 \mu \mathrm{m}$ (see Box 2.4), which allows the spermatozoa to swim freely:

- Place a standard volume of semen, e.g. $10 \mu \mathrm{l}$, onto a clean glass slide.

- Cover it with a coverslip, e.g. $22 \mathrm{~mm} \times 22 \mathrm{~mm}$ for $10 \mu \mathrm{l}$, to provide a chamber approximately $20 \mu \mathrm{m}$ deep (see Box 2.4 ). The weight of the coverslip spreads the sample.

- Take care to avoid the formation and trapping of air bubbles between the coverslip and the slide.

- Assess the freshly made wet preparation as soon as the contents are no longer drifting.

\section{Box 2.4 Depth of wet preparations}

The depth of a preparation $(D, \mu \mathrm{m})$ is obtained by dividing the volume of the sample $\left(\mathrm{V}, \mu \mathrm{l}=\mathrm{mm}^{3}\right)$ by the area over which it is spread $\left(A, \mathrm{~mm}^{2}\right): D=V / A$. Thus, a volume of $10 \mu \mathrm{l}$ of semen delivered onto a clean glass slide and covered with a $22 \mathrm{~mm} \times 22 \mathrm{~mm}$ coverslip (area $484 \mathrm{~mm}^{2}$ ) provides a chamber of depth of $20.7 \mu \mathrm{m}$; a $6.5 \mu \mathrm{l}$ sample covered with an $18 \mathrm{~mm} \times 18 \mathrm{~mm}$ coverslip (area $324 \mathrm{~mm}^{2}$ ) provides a depth of $20.1 \mu \mathrm{m}$; an $11 \mu \mathrm{l}$ sample covered by a $21 \mathrm{~mm} \times 26 \mathrm{~mm}$ coverslip (area $546 \mathrm{~mm}^{2}$ ) provides a depth of $20.1 \mu \mathrm{m}$. Occasionally, a deeper chamber may be required: a $40 \mu \mathrm{l}$ sample covered by a $24 \mathrm{~mm} \times 50 \mathrm{~mm}$ coverslip (area $1200 \mathrm{~mm}^{2}$ ) provides a depth of $33.3 \mu \mathrm{m}$.

Note 1: A chamber depth of less than $20 \mu \mathrm{m}$ constrains the rotational movement of spermatozoa (Le Lannou et al., 1992; Kraemer et al., 1998).

Note 2: If the chamber is too deep, it will be difficult to assess spermatozoa as they move in and out of focus. 
Note 3: If the number of spermatozoa per visual field varies considerably, the sample is not homogeneous. In such cases, the semen sample should be mixed again thoroughly (see Box 2.3) and a new slide prepared as above.

Note 4: Lack of homogeneity may also result from abnormal consistency, abnormal liquefaction (see Section 2.3.1), aggregation of spermatozoa (see Section 2.4.3) or sperm agglutination (see Section 2.4.4).

\subsubsection{Aggregation of spermatozoa}

The adherence either of immotile spermatozoa to each other or of motile spermatozoa to mucus strands, non-sperm cells or debris is considered to be nonspecific aggregation (Fig. 2.2) and should be recorded as such.

Fig. 2.2 Non-specific aggregation of spermatozoa in semen

Views of spermatozoa aggregated with an epithelial cell (a), debris (b) or spermatozoa (c, d).
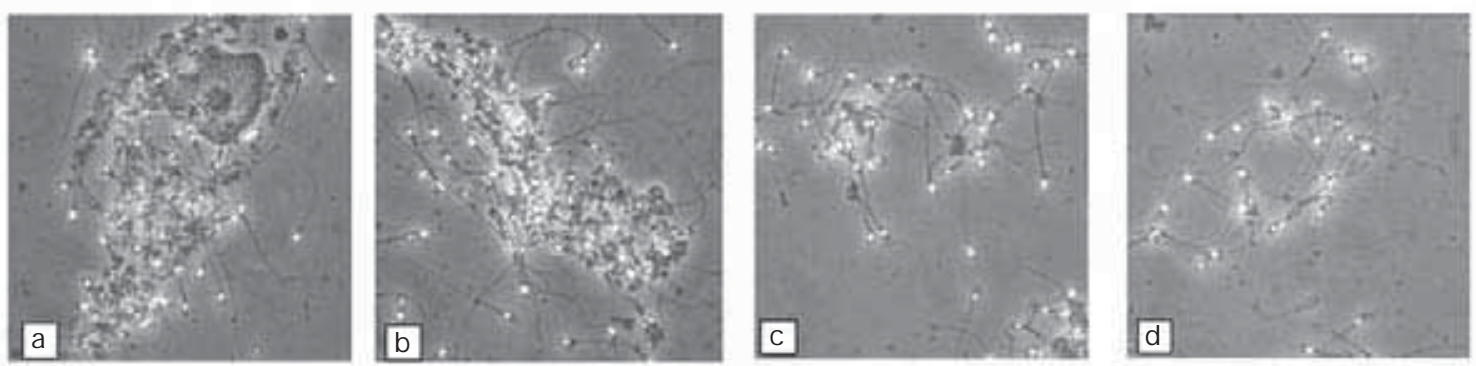

Micrographs courtesy of C Brazil.

\subsubsection{Agglutination of spermatozoa}

Agglutination specifically refers to motile spermatozoa sticking to each other, head-to-head, tail-to-tail or in a mixed way. The motility is often vigorous with a frantic shaking motion, but sometimes the spermatozoa are so agglutinated that their motion is limited. Any motile spermatozoa that stick to each other by their heads, tails or midpieces should be noted.

The major type of agglutination (reflecting the degree (grades 1-4) and the site of attachment (grades A-E) should be recorded (Rose et al., 1976) (see Fig. 2.3):

- grade 1: isolated

- grade 2: moderate

- grade 3: large

- grade 4: gross
$<10$ spermatozoa per agglutinate, many free spermatozoa

10-50 spermatozoa per agglutinate, free spermatozoa

agglutinates of $>50$ spermatozoa, some spermatozoa still free

all spermatozoa agglutinated and agglutinates interconnected 
Note: Motile spermatozoa stuck to cells or debris or immotile spermatozoa stuck to each other (aggregation) should not be scored as agglutination.

Fig. 2.3 Schematic diagram of different extents of sperm agglutination

Degree of agglutination

\begin{tabular}{llll}
\hline 1. Isolated & 2. Moderate & 3. Large (aggluti- & 4. Gross (all \\
(<10 sperm/ & (10-50 sperm/ & nates $>50$ sperm, & sperm agglu- \\
agglutinate, & agglutinate, & some sperm still & tinated, and \\
many free & free sperm) & free) & $\begin{array}{l}\text { agglutinates } \\
\text { interconnec- } \\
\text { sperm) }\end{array}$ \\
& & ted) \\
\end{tabular}

A. Head-to-head

B. Tail-to-tail (heads are seen to be free and move clear of agglutinates)

C. Tail-tip-to-tail-tip

D. Mixed (clear headto-head and tail-to-tail agglutinations)

E. Tangle (heads and tails enmeshed. Heads are not clear of agglutinates as they are in tailto-tail agglutination)
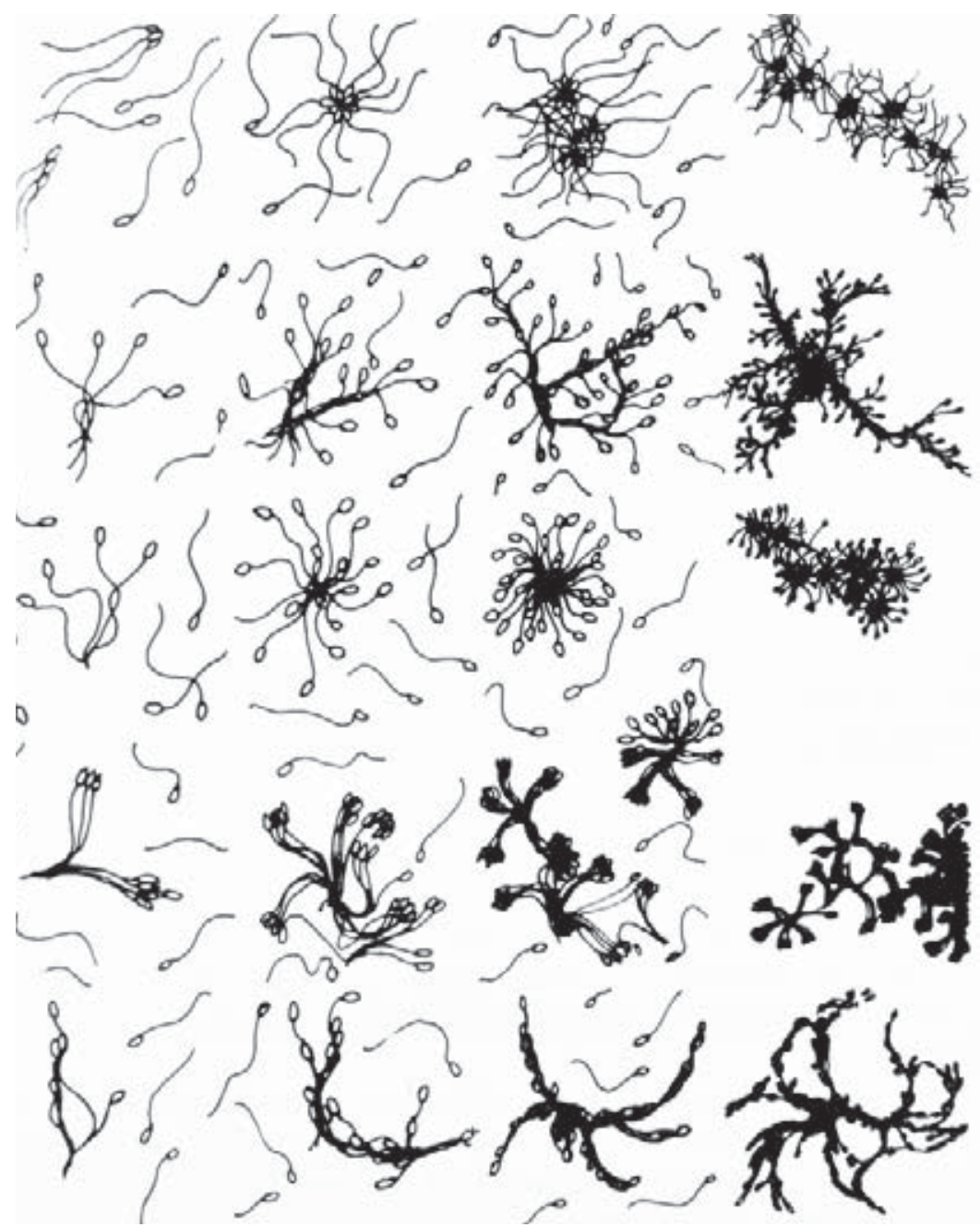

Reproduced from Rose et al. (1976) by permission of Wiley-B lackwell. 
Comment 1: The presence of agglutination is not sufficient evidence to deduce an immunological cause of infertility, but is suggestive of the presence of anti-sperm antibodies; further testing is required (see Section 2.20).

Comment 2: Severe agglutination can affect the assessment of sperm motility and concentration.

\subsubsection{Cellular elements other than spermatozoa}

The ejaculate contains cells other than spermatozoa, some of which may be clinically relevant. These include epithelial cells from the genitourinary tract, as well as leukocytes and immature germ cells, the latter two collectively referred to as "round cells" ( ohanisson et al., 2000). They can be identified by examining a stained smear at $\times 1000$ magnification (see Section 2.12, Plates 13 and 14, and Section 2.19). These cells can be more precisely identified and quantified by detecting peroxidase activity (see Section 2.18) or the antigen CD45 (see Section 3.2). Their concentration can be estimated as for spermatozoa, from wet preparations (see Section 2.18.1.5) or from the ratio of these cells to the number of spermatozoa on the stained smear and the sperm concentration (see Section 2.12.1).

\subsection{Sperm motility}

The extent of progressive sperm motility (see Section 2.5.1) is related to pregnancy rates (J ouannet et al., 1988; Larsen et al., 2000; Zinaman et al., 2000). Methods of motility assessment involving computer-aided sperm analysis (CASA) are described in Section 3.5.2.

Sperm motility within semen should be assessed as soon as possible after liquefaction of the sample, preferably at 30 minutes, but in any case within 1 hour, following ejaculation, to limit the deleterious effects of dehydration, $\mathrm{pH}$ or changes in temperature on motility.

- Mix the semen sample well (see Box 2.3).

- Remove an aliquot of semen immediately after mixing, allowing no time for the spermatozoa to settle out of suspension.

- Remix the semen sample before removing a replicate aliquot.

- For each replicate, prepare a wet preparation approximately $20 \mu \mathrm{m}$ deep (see Section 2.4.2).

- Wait for the sample to stop drifting (within 60 seconds).

- Examine the slide with phase-contrast optics at $\times 200$ or $\times 400$ magnification.

- Assess approximately 200 spermatozoa per replicate for the percentage of different motile categories.

- Compare the replicate values to check if they are acceptably close. If so, proceed with calculations; if not, prepare new samples. 
Note 1: The procedure may be performed at room temperature or at $37^{\circ} \mathrm{C}$ with a heated microscope stage, but should be standardized for each laboratory. If sperm motility is to be assessed at $37^{\circ} \mathrm{C}$, the sample should be incubated at this temperature and the preparation made with prewarmed slides and coverslips.

Note 2: The use of an eyepiece reticle with grid (see Fig. 2.4a) is recommended to limit the area viewed; this allows the same area of the slide to be assessed during both stages of scoring. Assess progressive motility first, then non-progressive motility and immotility (see Section 2.5.1). Limiting the area, and thus the number of spermatozoa assessed, ensures that several areas of the preparation are examined for motility.

\subsubsection{Categories of sperm movement}

A simple system for grading motility is recommended that distinguishes spermatozoa with progressive or non-progressive motility from those that are immotile. The motility of each spermatozoon is graded as follows:

- Progressive motility (PR): spermatozoa moving actively, either linearly or in a large circle, regardless of speed.

- Non-progressive motility (NP): all other patterns of motility with an absence of progression, e.g. swimming in small circles, the flagellar force hardly displacing the head, or when only a flagellar beat can be observed.

- Immotility (IM): no movement.

Comment 1: The previous edition of this manual recommended that progressively motile spermatozoa should be categorized as rapid or slow, with a speed of $>25 \mu \mathrm{m} / \mathrm{sec}$ at $37^{\circ} \mathrm{C}$ defining "grade a" spermatozoa. However, it is difficult for technicians to define the forward progression so accurately without bias (Cooper \& Yeung, 2006).

Comment 2: When discussing sperm motility, it is important to specify total motility $(P R+N P)$ or progressive motility (PR).

\subsubsection{Preparing and assessing a sample for motility}

- If motility is to be assessed at $37^{\circ} \mathrm{C}$, turn the stage warmer on 10 minutes in advance, to allow the temperature to stabilize.

- Prepare a wet preparation $20 \mu \mathrm{m}$ deep (see Section 2.4.2).

- Examine the slide with phase-contrast optics at $\times 200$ or $\times 400$ magnification.

- Wait for the sample to stop drifting.

- Look for spermatozoa in an area at least $5 \mathrm{~mm}$ from the edge of the coverslip (see Fig. 2.4b), to prevent observation of effects of drying on motility. 
- Systematically scan the slide to avoid repeatedly viewing the same area. Change fields often. Avoid choosing fields on the basis of the number of motile sperm seen (field choice should be random).

- Start scoring a given field at a random instant. Do not wait for spermatozoa to swim into the field or grid to begin scoring.

- Assess the motility of all spermatozoa within a defined area of the field. This is most easily achieved by using an eyepiece reticle (see Fig. 2.4a). Select the portion of the field or grid to be scored from the sperm concentration, i.e. score only the top row of the grid if the sperm concentration is high; score the entire grid if the sperm concentration is low.

- Scan and count quickly to avoid overestimating the number of motile spermatozoa. The goal is to count all motile spermatozoa in the grid section instantly; avoid counting both those present initially plus those that swim into the grid section during scoring, which would bias the result in favour of motile spermatozoa.

- Initially scan the grid section being scored for PR cells (see Section 2.5.1). Next count NP spermatozoa and finally IM spermatozoa in the same grid section. With experience, it may be possible to score all three categories of sperm movement at one time, and to score larger areas of the grid.

- Tally the number of spermatozoa in each motility category with the aid of a laboratory counter.

- Evaluate at least 200 spermatozoa in a total of at least five fields in each replicate, in order to achieve an acceptably low sampling error (see Box 2.5).

- Calculate the average percentage and difference between the two percentages for the most frequent motility grade (PR, NP or IM) in the replicate wet preparations.

- Determine the acceptability of the difference from Table 2.1 or Fig. A7.2, Appendix 7. (Each shows the maximum difference between two percentages that is expected to occur in $95 \%$ of samples because of sampling error alone.)

- If the difference between the percentages is acceptable, report the average percentage for each motility grade (PR, NP and IM). If the difference is too high, take two new aliquots from the semen sample, make two new preparations and repeat the assessment (see Box 2.6).

- Report the average percentage for each motility grade to the nearest whole number.

Note 1: Assess only intact spermatozoa (defined as having a head and a tail; see Section 2.7.3), since only intact spermatozoa are counted for sperm concentration. Do not count motile pinheads. 
Note 2: If spermatozoa are being scored in two stages (i.e. PR first, followed by NP and IM from the same area) and a count of 200 spermatozoa is achieved before all motility categories from that area have been scored, counting must continue beyond 200 spermatozoa until all categories have been counted, in order to avoid bias towards the motility category scored first.

Note 3: It is common to overestimate sperm motility, but this can often be avoided by reversing the order of analysis (NP and IM first), using an eyepiece reticle, and being aware of, and avoiding, to the extent possible, potential sources of bias (see Section 7.13.3).

Note 4: On rare occasions, with inhomogeneous samples, even a third set of replicates may provide unacceptable differences. In this case, calculate the mean of all replicates and note this in the report.

Fig. 2.4 Aids to assessing sperm motility

(a) An eyepiece reticle makes it easier to count motile and immotile spermatozoa. (b) Systematic selection of fields for assessment of sperm motility, at least $5 \mathrm{~mm}$ from the edges of the coverslip.

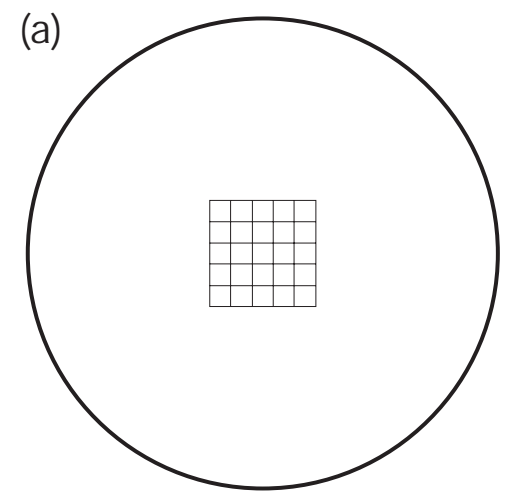

(b)

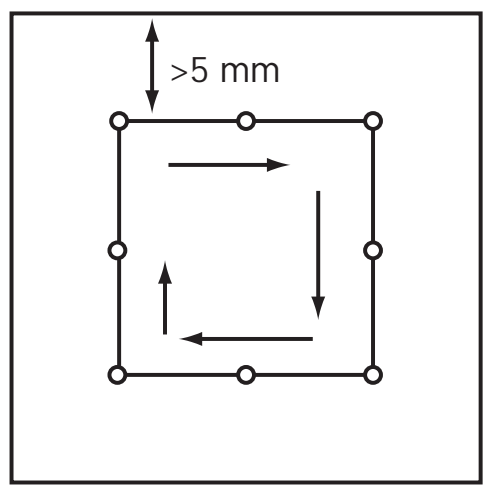

\section{Box 2.5 Errors in estimating percentages}

How certain your estimate of a percentage is depends not only on the number $(N)$ of spermatozoa counted but also on the true, but unknown, percentage $(p)$ (binomial distribution). The approximate standard error (SE) is $\sqrt{ }((p(100-p)) / N)$ for percentages between 20 and 80 . Outside this range, a more appropriate method to use is the angular transformation (arc sin square root), $z=\sin ^{-1} \sqrt{(p / 100)}$, with a standard deviation of $1 /(2 \sqrt{N})$ radians, which depends only on the number of spermatozoa counted and not the true percentage. 
Table 2.1 Acceptable differences between two percentages for a given average, determined from replicate counts of 200 spermatozoa (total 400 counted)

\begin{tabular}{|c|c|}
\hline Average (\%) & $\begin{array}{c}\text { Acceptable } \\
\text { Difference* }\end{array}$ \\
\hline 0 & 1 \\
\hline 1 & 2 \\
\hline 2 & 3 \\
\hline $3-4$ & 4 \\
\hline $5-7$ & 5 \\
\hline $8-11$ & 6 \\
\hline $12-16$ & 7 \\
\hline $17-23$ & 8 \\
\hline $24-34$ & 9 \\
\hline $35-65$ & 10 \\
\hline
\end{tabular}

\begin{tabular}{|c|c|}
\hline Average (\%) & $\begin{array}{c}\text { Acceptable } \\
\text { Difference* }\end{array}$ \\
\hline $66-76$ & 9 \\
\hline $77-83$ & 8 \\
\hline $84-88$ & 7 \\
\hline $89-92$ & 6 \\
\hline $93-95$ & 5 \\
\hline $96-97$ & 4 \\
\hline 98 & 3 \\
\hline 99 & 2 \\
\hline 100 & 1 \\
\hline
\end{tabular}

*Based on the rounded $95 \%$ confidence interval.

\section{B ox 2.6 Comparison of replicate percentages}

Percentages should be rounded to the nearest whole number. The convention is to round $0.5 \%$ to the nearest even number, e.g. $32.5 \%$ is rounded down to $32 \%$ but $3.5 \%$ is rounded up to $4 \%$. Note that the rounded percentages may not add up to $100 \%$. If the difference between the replicate percentages is less than or equal to that indicated in Table 2.1 for the given average, the estimates are accepted and the average is taken as the result.

Larger than acceptable differences suggest that there has been miscounting or errors of pipetting, or that the cells were not mixed well, with non-random distribution in the chamber or on the slide.

When the difference between percentages is greater than acceptable, discard the first two values and reassess. (Do not count a third sample and take the mean of the three values, or take the mean of the two closest values.)

For estimates of sperm motility, or vitality by eosin alone and for the hypo-osmotic swelling (HOS) test, prepare fresh replicates from new aliquots of semen. For estimates of vitality from eosin-nigrosin smears and sperm morphology, reassess the slides in replicate.

With these $95 \% \mathrm{Cl}$ cut-off values, approximately $5 \%$ of replicates will be outside the limits by chance alone (see Appendix 7, section A7.3). Exact binomial confidence limits can now be computer-generated, and these are used in this manual for the graphs and tables provided to assess agreement of replicates.

\subsubsection{Worked examples}

Example 1. Sperm motility estimates in replicate counts of 200 spermatozoa are: progressive, $30 \%$ and $50 \%$; non-progressive, $5 \%$ and $15 \%$; immotile, $65 \%$ and 
$35 \%$. The most common category is immotile, with an average of $50 \%$ and a difference of $30 \%$. From Table 2.1, it is seen that for an average of $50 \%$, a difference of up to $10 \%$ would be expected to occur by chance alone. As the observed difference exceeds this, the results are discarded and two fresh slides are prepared and the sperm motility re-estimated.

Example 2. Sperm motility estimates in replicate counts of 200 spermatozoa are: progressive, $37 \%$ and $28 \%$; non-progressive, $3 \%$ and $6 \%$; immotile $60 \%$ and $66 \%$. The most common category is immotile, with an average of $63 \%$ and a difference of $6 \%$. From Table 2.1, it is seen that for an average of $63 \%$, a difference of up to $10 \%$ would be expected to occur by chance alone. As the observed difference is less than this, the results are accepted and the mean values reported: PR 32\%, NP 4\%, IM 63\%.

\subsubsection{Lower reference limit}

The lower reference limit for total motility $(\mathrm{PR}+\mathrm{NP})$ is $40 \%$ (5th centile, $95 \% \mathrm{Cl}$ 38-42).

The lower reference limit for progressive motility $(P R)$ is $32 \%$ (5th centile, $95 \% \mathrm{Cl}$ 31-34).

Comment: The total number of progressively motile spermatozoa in the ejaculate is of biological significance. This is obtained by multiplying the total number of spermatozoa in the ejaculate (see Section 2.8 .7 ) by the percentage of progressively motile cells.

\subsection{Sperm vitality}

Sperm vitality, as estimated by assessing the membrane integrity of the cells, may be determined routinely on all samples, but is especially important for samples with less than about $40 \%$ progressively motile spermatozoa. This test can provide a check on the motility evaluation, since the percentage of dead cells should not exceed (within sampling error) the percentage of immotile spermatozoa. The percentage of viable cells normally exceeds that of motile cells.

The percentage of live spermatozoa is assessed by identifying those with an intact cell membrane, from dye exclusion or by hypotonic swelling. The dye exclusion method is based on the principle that damaged plasma membranes, such as those found in non-vital (dead) cells, allow entry of membrane-impermeant stains. The hypo-osmotic swelling test presumes that only cells with intact membranes (live cells) will swell in hypotonic solutions. Examples of each test are described below.

Sperm vitality should be assessed as soon as possible after liquefaction of the semen sample, preferably at 30 minutes, but in any case within 1 hour of ejaculation, to prevent observation of deleterious effects of dehydration or of changes in temperature on vitality. 
Comment 1: It is clinically important to know whether immotile spermatozoa are alive or dead. Vitality results should be assessed in conjunction with motility results from the same semen sample.

Comment 2: The presence of a large proportion of vital but immotile cells may be indicative of structural defects in the flagellum (Chemes $\&$ Rawe, 2003); a high percentage of immotile and non-viable cells (necrozoospermia) may indicate epididymal pathology (Wilton et al., 1988; Correa-Perez et al., 2004).

2.6.1 Vitality test using eosin-nigrosin

This one-step staining technique uses nigrosin to increase the contrast between the background and the sperm heads, which makes them easier to discern. It also permits slides to be stored for re-evaluation and quality-control purposes (Björndahl et al., 2003).

\subsubsection{Preparing the reagents}

1. Eosin $Y$ : dissolve $0.67 \mathrm{~g}$ of eosin $Y$ (colour index 45380 ) and $0.9 \mathrm{~g}$ of sodium chloride $(\mathrm{NaCl})$ in $100 \mathrm{ml}$ of purified water with gentle heating.

2. Eosin-nigrosin: add $10 \mathrm{~g}$ of nigrosin (colour index 50420) to the $100 \mathrm{ml}$ of eosin Y solution.

3. Boil the suspension, then allow to cool to room temperature.

4. Filter through filter paper (e.g. $\left.90 \mathrm{~g} / \mathrm{m}^{2}\right)$ to remove coarse and gelatinous precipitates and store in a sealed dark-glass bottle.

\subsubsection{Procedure}

1. Mix the semen sample well (see Box 2.3).

2. Remove a 50- $\mu$ l aliquot of semen and mix with an equal volume of eosinnigrosin suspension, e.g. in a porcelain spot plate well or test-tube, and wait for 30 seconds.

3. Remix the semen sample before removing a replicate aliquot and mixing with eosin-nigrosin and treating as in step 2 above.

4. For each suspension make a smear on a glass slide (see Section 2.13.2) and allow it to dry in air.

5. Examine immediately after drying, or later after mounting with a permanent non-aqueous mounting medium (see Section 2.14.2.5).

6. Examine each slide with brightfield optics at $\times 1000$ magnification and oil immersion.

7. Tally the number of stained (dead) or unstained (vital) cells with the aid of a laboratory counter. 
8. Evaluate 200 spermatozoa in each replicate, in order to achieve an acceptably low sampling error (see Box 2.5).

9. Calculate the average and difference of the two percentages of vital cells from the replicate slides.

10. Determine the acceptability of the difference from Table 2.1 or Fig. A7.2, Appendix 7. (Each shows the maximum difference between two percentages that is expected to occur in $95 \%$ of samples because of sampling error alone.)

11. If the difference between the percentages is acceptable, report the average percentage of vital spermatozoa. If the difference is too high, make two new preparations from two fresh aliquots of the semen sample and repeat the assessment (see Box 2.6).

12. Report the average percentage of vital spermatozoa to the nearest whole number.

Fig. 2.5 Eosin-nigrosin smear observed in brightfield optics

Spermatozoa with red (D1) or dark pink (D2) heads are considered dead (membrane-damaged), whereas spermatozoa with white heads $(L)$ or light pink heads are considered alive (membraneintact).

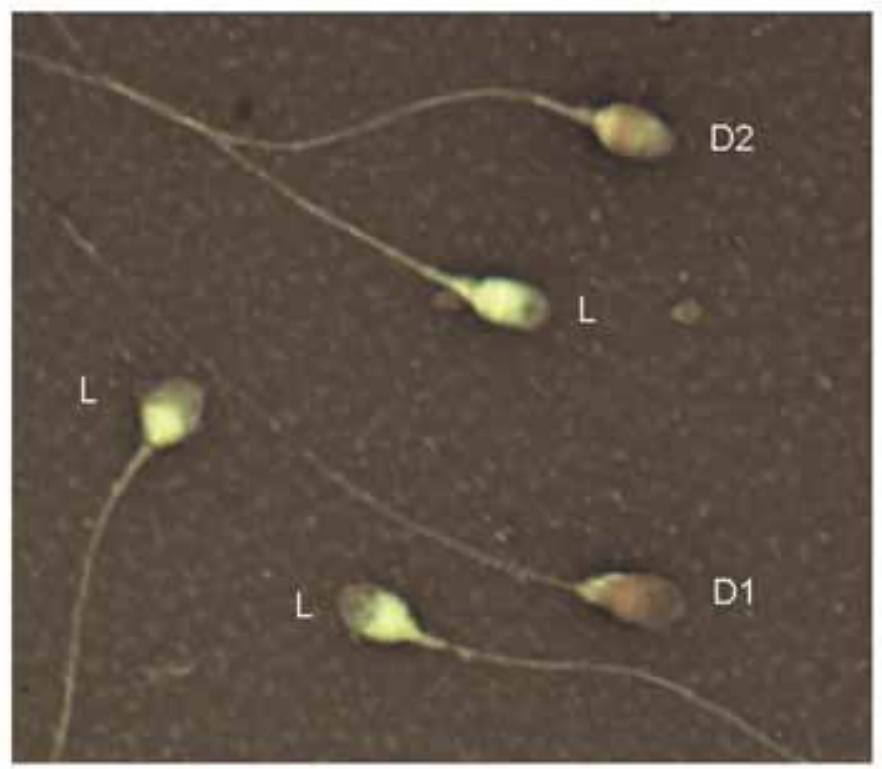

Micrograph courtesy of TG Cooper.

\subsubsection{Scoring}

1. The nigrosin provides a dark background that makes it easier to discern faintly stained spermatozoa. 
2. With brightfield optics, live spermatozoa have white heads and dead spermatozoa have heads that are stained red or dark pink (see Fig. 2.5). Spermatozoa with a faint pink head are assessed as alive.

3. If the stain is limited to only a part of the neck region, and the rest of the head area is unstained, this is considered a "leaky neck membrane", not a sign of cell death and total membrane disintegration. These cells should be assessed as alive.

\subsubsection{Lower reference limit}

The lower reference limit for vitality (membrane-intact spermatozoa) is $58 \%$ (5th centile, 95\% Cl 55-63).

Comment: The total number of membrane-intact spermatozoa in the ejaculate is of biological significance. This is obtained by multiplying the total number of spermatozoa in the ejaculate (see Section 2.8.7) by the percentage of membrane-intact cells.

\subsubsection{Vitality test using eosin alone}

This method is simple and rapid, but the wet preparations cannot be stored for quality control purposes.

\subsubsection{Preparing the reagents}

1. $\mathrm{NaCl}, 0.9 \%(\mathrm{w} / \mathrm{v})$ : dissolve $0.9 \mathrm{~g}$ of $\mathrm{NaCl}$ in $100 \mathrm{ml}$ purified water.

2. Eosin $Y, 0.5 \%(w / v)$ : dissolve $0.5 \mathrm{~g}$ of eosin $Y$ (colour index 45380 ) in $100 \mathrm{ml}$ of $0.9 \% \mathrm{NaCl}$.

Note: Some commercially available eosin solutions are hypotonic aqueous solutions that will stress the spermatozoa and give false-positive results (Björndahl et al., 2004). If using such a solution, add $0.9 \mathrm{~g}$ of $\mathrm{NaCl}$ to $100 \mathrm{ml}$ of solution to raise the osmolality.

\subsubsection{Procedure}

1. Mix the semen sample well (see Box 2.3).

2. Remove an aliquot of $5 \mu$ l of semen and combine with $5 \mu$ l of eosin solution on a mic roscope slide. Mix with a pipette tip, swirling the sample on the slide.

3. Cover with a $22 \mathrm{~mm} \times 22 \mathrm{~mm}$ coverslip and leave for 30 seconds.

4. Remix the semen sample, remove a replicate aliquot, mix with eosin and treat as in steps 2 and 3 above.

5. Examine each slide, preferably with negative-phase-contrast optics (positivephase-contrast makes faint pink heads difficult to discern) at $\times 200$ or $\times 400$ magnification. 
6. Tally the number of stained (dead) and unstained (vital) cells with the aid of a laboratory counter.

7. Evaluate 200 spermatozoa in each replicate, in order to achieve an acceptably low sampling error (see Box 2.5).

8. Calculate the average and difference of the two percentages of vital cells from the replicate preparations.

9. Determine the acceptability of the difference from Table 2.1 or Fig. A7.2, Appendix 7. (Each shows the maximum difference between two percentages that is expected to occur in $95 \%$ of samples because of sampling error alone.)

10. If the difference between the percentages is acceptable, report the average percentage vitality. If the difference is too high, make two new preparations from two new aliquots of semen and repeat the assessment (see Box 2.6).

11. Report the average percentage of vital spermatozoa to the nearest whole number.

\subsubsection{Scoring}

1. Live spermatozoa have white or light pink heads and dead spermatozoa have heads that are stained red or dark pink.

2. If the stain is limited to only a part of the neck region, and the rest of the head area is unstained, this is considered a "leaky neck membrane", not a sign of cell death and total membrane disintegration. These cells should be assessed as alive.

3. If it is difficult to discern the pale pink stained head, use nigrosin to increase the contrast of the background (see Section 2.6.1).

\subsubsection{Lower reference limit}

The lower reference limit for vitality (membrane-intact spermatozoa) is $58 \%$ (5th centile, 95\% CI 55-63).

Comment: The total number of membrane-intact spermatozoa in the ejaculate is of biological significance. This is obtained by multiplying the total number of spermatozoa in the ejaculate (see Section 2.8.7) by the percentage of membrane-intact cells.

2.6.3 Vitality test using hypo-osmotic swelling

As an alternative to dye exclusion, the hypo-osmotic swelling (HOS) test may be used to assess vitality ( eyendran et al., 1984). This is useful when staining of spermatozoa must be avoided, e.g. when choosing spermatozoa for ICSI. Spermatozoa with intact membranes swell within 5 minutes in hypo-osmotic medium and all flagellar shapes are stabilized by 30 minutes (Hossain et al., 1998). 
Thus, use:

- 30 minutes incubation for routine diagnostics; but

- 5 minutes incubation when spermatozoa are to be processed for therapeutic use.

\subsubsection{Preparing the reagents}

1. Swelling solution for diagnostic purposes: dissolve $0.735 \mathrm{~g}$ of sodium citrate dihydrate and $1.351 \mathrm{~g}$ of $\mathrm{D}$-fructose in $100 \mathrm{ml}$ of purified water. Freeze 1 - $\mathrm{ml}$ aliquots of this solution at $-20^{\circ} \mathrm{C}$.

2. For therapeutic use: dilute the medium to be used $1+1(1: 2)$ with sterile, purified water.

\subsubsection{Procedure}

1. Thaw the frozen swelling solution and mix well before use.

2. Warm $1 \mathrm{ml}$ of swelling solution or $1 \mathrm{ml}$ of $1+1(1: 2)$ diluted medium in a closed microcentrifuge tube at $37^{\circ} \mathrm{C}$ for 5 minutes.

3. Mix the semen sample well (see Box 2.3).

4. Remove a 100- $\mu$ l aliquot of semen and add to the swelling solution. Mix gently by drawing it in and out of the pipette.

5. Incubate at $37^{\circ} \mathrm{C}$ for exactly 5 minutes or 30 minutes (see above), then transfer a $10-\mu \mathrm{l}$ aliquot to a clean slide and cover with a $22 \mathrm{~mm} \times 22 \mathrm{~mm}$ coverslip.

6. Remix the semen sample, remove a replicate aliquot, mix with swelling solution, incubate and prepare a replicate slide, as above.

7. Examine each slide with phase-contrast optics at $\times 200$ or $\times 400$ magnification.

8. Tally the number of unswollen (dead) and swollen (vital) cells with the aid of a laboratory counter.

9. Evaluate 200 spermatozoa in each replicate, in order to achieve an acceptably low sampling error (see Box 2.5).

10. Calculate the average and difference of the two percentages of vital cells from the replicate preparations.

11. Determine the acceptability of the difference from Table 2.1 or Fig. A7.2, Appendix 7. (Each shows the maximum difference between two percentages that is expected to occur in $95 \%$ of samples because of sampling error alone.)

12. If the difference between the percentages is acceptable, report the average percentage vitality. If the difference is too high, make two new preparations from two new aliquots of semen and repeat the assessment (see Box 2.6).

13. Report the average percentage of vital spermatozoa to the nearest whole number. 


\subsubsection{Scoring}

1. Swollen spermatozoa are identified by changes in the shape of the cell, as indicated by coiling of the tail (Fig. 2.6).

2. Live cells are distinguished by evidence of swelling of the sperm tail; score all forms of swollen tails as live spermatozoa.

Fig. 2.6 Schematic representation of typical morphological changes in human spermatozoa subjected to hypo-osmotic stress

(a) No change. (b)- (g) Various types of tail changes. Swelling in tail is indicated by the grey area.

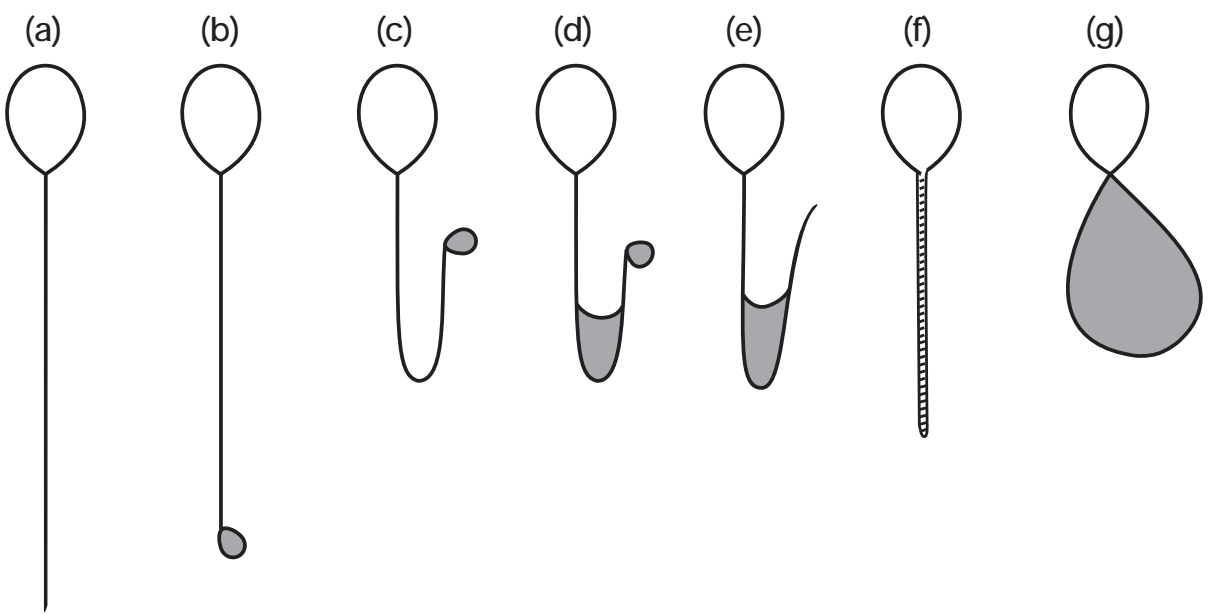

Reproduced from J eyendran RS, Van der Ven HH, Perez-Pelaez M, Crabo BG, Zaneveld LJ D. (1984) J ournal of Reproduction and Fertility, 70: 219-228. (c) Society for Reproduction and Fertility (1984). Reproduced by permission.

\subsubsection{Lower reference limit}

HOS test values approximate those of the eosin test (Carreras et al., 1992).

The lower reference limit for vitality (membrane-intact spermatozoa) is $58 \%$ (5th centile, 95\% CI 55-63).

Comment: The total number of membrane-intact spermatozoa in the ejaculate is of biological significance. This is obtained by multiplying the total number of spermatozoa in the ejaculate (see Section 2.8.7) by the percentage of membrane-intact cells.

\subsection{Sperm numbers}

The total number of spermatozoa per ejaculate and the sperm concentration are related to both time to pregnancy (Slama et al., 2002) and pregnancy rates (WHO, 1996; Zinaman et al., 2000) and are predictors of conception (Bonde et al., 1998; 
Larsen et al., 2000). More data correlating total sperm numbers with reproductive outcome are warranted.

The number of spermatozoa in the ejaculate is calculated from the concentration of spermatozoa, which is measured during semen evalulation. For normal ejaculates, when the male tract is unobstructed and the abstinence time short, the total number of spermatozoa in the ejaculate is correlated with testicular volume (Handelsman et al., 1984; WHO, 1987; Andersen et al., 2000; Behre et al., 2000) and thus is a measure of the capability of the testes to produce spermatozoa (MacLeod \& Wang, 1979) and the patency of the male tract. The concentration of spermatozoa in the semen, while related to fertilization and pregnancy rates, is influenced by the volume of the secretions from the seminal vesicles and prostate (Eliasson, 1975) and is not a specific measure of testicular function.

Comment 1: The terms "total sperm number" and "sperm concentration" are not synonymous. Sperm concentration refers to the number of spermatozoa per unit volume of semen and is a function of the number of spermatozoa emitted and the volume of fluid diluting them. Total sperm number refers to the total number of spermatozoa in the entire ejaculate and is obtained by multiplying the sperm concentration by the semen volume.

Comment 2: The generalization that total sperm number reflects testicular sperm productivity may not hold for electro-ejaculates from men with spinal cord injury, those with androgen deficiency, or for samples collected after prolonged abstinence or partial retrograde ejaculation.

Comment 3: The term "sperm density" (mass per unit volume) should not be used when sperm concentration (number per unit volume) is meant.

Determination of sperm number comprises the following steps (which are described in detail in subsequent sections).

- Examining a well-mixed, undiluted preparation of liquefied semen on a glass slide under a coverslip, to determine the appropriate dilution and appropriate chambers to use (see Section 2.8.1). This is usually the wet preparation (see Section 2.4.2) used for evaluation of motility.

- Mixing semen and preparing dilutions with fixative.

- Loading the haemocytometer chamber and allowing spermatozoa to settle in a humid chamber.

- Assessing the samples within 10-15 minutes (after which evaporation has noticeable effects on sperm position within the chamber).

- Counting at least 200 spermatozoa per replicate.

- Comparing replicate counts to see if they are acceptably close. If so, proceeding with calculations; if not, preparing new dilutions.

- Calculating the concentration in spermatozoa per ml.

- Calculating the total number of spermatozoa per ejaculate. 


\subsubsection{Types of counting chamber}

The use of 100- $\mu \mathrm{m}$-deep haemocytometer chambers is recommended. Dilution factors for the improved Neubauer haemocytometer chamber are given here. Other deep haemocytometer chambers may be used, but they will have different volumes and grid patterns and will require different factors for calculation. Disposable chambers are available for determining sperm concentration (Seaman et al., 1996; Mahmoud et al., 1997; B razil et al., 2004b), but they may produce different results from those of the improved Neubauer haemocytometer. Shallow chambers that fill by capillary action may not have a uniform distribution of spermatozoa because of streaming (Douglas-Hamilton et al., 2005a, 2005b). It may be possible to correct for this (Douglas-Hamilton et al., 2005a) but it is not advised (Björndahl \& Barratt, 2005). The validity of these alternative counting chambers must be established by checking chamber dimensions (see Appendix 7, section A7.8), comparing results with the improved Neubauer haemocytometer method, and obtaining satisfactory performance as shown by an external quality-control programme. For accurate assessment of low sperm concentrations, large-volume counting chambers may be necessary (see Section 2.11.2).

\subsubsection{The improved Neubauer haemocytometer}

The improved Neubauer haemocytometer has two separate counting chambers, each of which has a microscopic $3 \mathrm{~mm} \times 3 \mathrm{~mm}$ pattern of gridlines etched on the glass surface. It is used with a special thick coverslip (thickness number 4 , $0.44 \mathrm{~mm}$ ), which lies over the grids and is supported by glass pillars $0.1 \mathrm{~mm}$ above the chamber floor. Each counting area is divided into nine $1 \mathrm{~mm} \times 1 \mathrm{~mm}$ grids. These grids are referred to by the numbers shown in Fig. 2.7.

Fig. 2.7 The improved Neubauer haemocytometer

Sketches of the inscribed area showing: all nine grids in one chamber of the haemocytometer (left panel); the central grid (number 5) of 25 large squares (middle panel); and a micrograph of part of a filled chamber (right panel), showing one of the 25 squares of the central grid (the circled square in the middle panel) bounded by triple lines and containing 16 smaller squares.
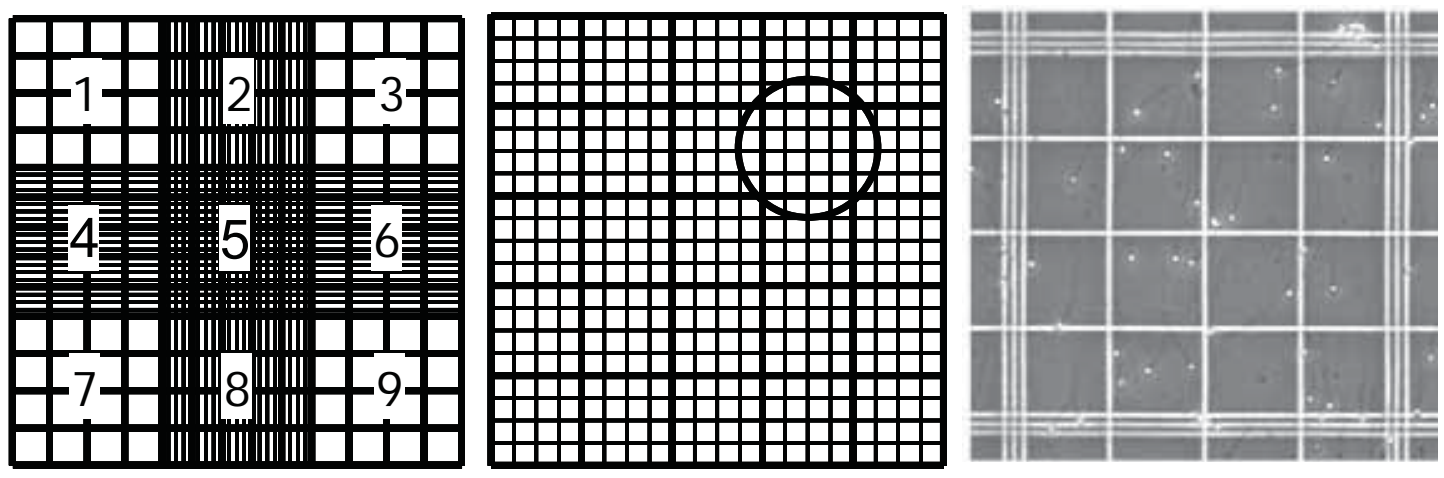

Micrograph courtesy of C Brazil. 
With a depth of $100 \mu \mathrm{m}$, each grid holds $100 \mathrm{nl}$. Four of these grids (nos 1, 3, 7 and 9) contain four rows of four squares, each holding $6.25 \mathrm{nl}$; two grids (nos 2 and 8) contain four rows of five squares, each of $5 \mathrm{nl}$; two grids (nos 4 and 6) contain five rows of four squares, each of $5 \mathrm{nl}$; and the central grid (number 5 ) contains five rows of five squares, each of $4 \mathrm{nl}$ (Fig. 2.7, middle panel). Each of the 25 squares of the central grid (number 5 ) is subdivided into 16 smaller squares (Fig. 2.7, right panel). Thus, grids 1, 2, 3, 7, 8 and 9 each have four rows holding $25 \mathrm{nl}$ per row, while grids 4,5 and 6 each have five rows holding $20 \mathrm{nl}$ per row.

Depending on the dilution and the number of spermatozoa counted, different areas of the chamber are used for determining sperm concentration. For $1+19$ $(1: 20)$ and $1+4(1: 5)$ dilutions, rows from grid number 5 are assessed and, when necessary, from grids numbers 4 and 6 (see Section 2.8). For $1+1$ (1:2) dilutions, all nine grids can be assessed if necessary to achieve a count of 200 spermatozoa (see Section 2.11.1).

2.7.3 Using the haemocytometer grid

- Count only whole spermatozoa (with heads and tails).

- Whether or not a spermatozoon is counted is determined by the location of its head; the orientation of its tail is unimportant. The boundary of a square is indicated by the middle line of the three; thus, a spermatozoon is counted if most of its head lies between the two inner lines, but not if most of its head lies between the two outer lines (Fig. 2.8, left panel).

- To avoid counting the same spermatozoon in adjacent squares, a spermatozoon with its head on the line dividing two adjacent squares should be counted only if that line is one of two perpendicular boundary lines. For example, cells may be counted if most of the sperm head lies on the lower or left centre boundaries, which form an "L" shape (see Fig. 2.8, middle panel), but not if it lies on the upper or right centre boundary line (Fig. 2.8, right panel).

Note: If there are many headless sperm tails (pinheads) or heads without tails, their presence should be recorded in the report. If considered necessary, their concentration can be assessed in the same way as for spermatozoa (see Section 2.8), or their prevalence relative to spermatozoa can be determined from stained preparations (see Section 2.17.6).

\subsubsection{Care of the counting chamber}

Haemocytometer counting chambers must be used with the special thick coverslips (thickness number $4,0.44 \mathrm{~mm}$ ).

- Clean the haemocytometer chamber and coverslip with water and dry well with tissue after use, as any dried residue can inhibit loading. Rubbing the grid surface will remove any residual spermatozoa from the previous sample.

- Soak reusable chambers and coverslips overnight in disinfectant (see Appendix 2, section A2.4) to avoid contamination with potentially infectious agents in semen. 
Fig. 2.8 Which spermatozoa to count in the grid squares

The middle of the three lines defines the square's boundary (black line, left panel). All spermatozoa within the central square are counted, as well as those with their heads between the two inner lines (white circles), but not those whose heads lie between the outer two lines (black circles). A spermatozoon with most of its head lying on the central line is counted only if that line is the lower or left-hand line of the square (white circles, middle panel) but not if it is the upper or right hand line of the square (black circles, right panel).
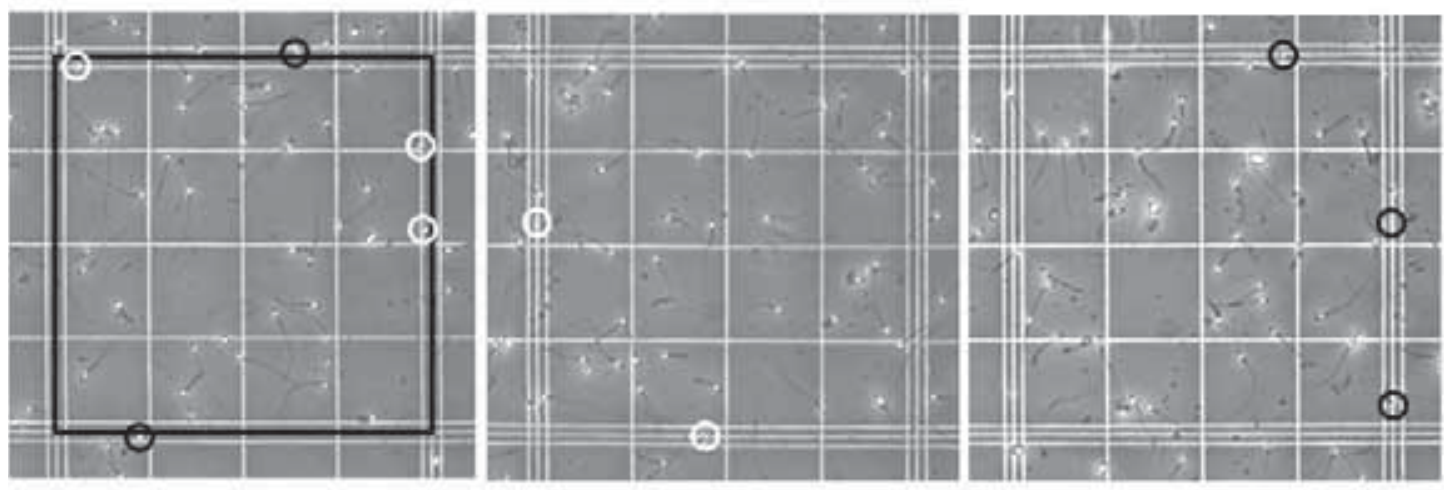

Micrographs courtesy of C Brazil.

\subsubsection{Fixative for diluting semen}

1. Dissolve $50 \mathrm{~g}$ of sodium bicarbonate $\left(\mathrm{NaHCO}_{3}\right.$ ) and $10 \mathrm{ml}$ of $35 \%(\mathrm{v} / \mathrm{v})$ formalin in $1000 \mathrm{ml}$ of purified water.

2. If desired, add $0.25 \mathrm{~g}$ of trypan blue (colour index 23859 ) or $5 \mathrm{ml}$ of saturated ( $>4 \mathrm{mg} / \mathrm{ml}$ ) gentian violet (colour index 42555 ) to highlight the sperm heads.

3. Store at $4{ }^{\circ} \mathrm{C}$. If crystals form in the solution, pass it through a $0.45-\mu \mathrm{m}$ filter before use.

\subsubsection{Importance of counting sufficient spermatozoa}

To reduce sampling errors, a critical number of spermatozoa have to be counted (preferably a total of at least 400 , from replicate counts of approximately 200 ) (see Box 2.7 and Table 2.2).

\section{Box 2.7 Errors in estimating numbers}

The precision of the estimate of sperm number depends on the number of spermatozoa counted. In a Poisson distribution, the standard error (SE) of a count (N) is its square root $(\sqrt{ } \mathrm{N})$ and the $95 \%$ confidence interval $(\mathrm{CI})$ for the number of spermatozoa in the volume of semen is approximately $\mathrm{N} \pm 1.96 \times \sqrt{ } \mathrm{N}$ (or $\mathrm{N} \pm$ approximately $2 \times \sqrt{ } \mathrm{N})$.

If 100 spermatozoa are counted, the SE is $10(\sqrt{ } 100)$, and the $95 \% \mathrm{Cl}$ is $80-120$ $(100 \pm 20)$. If 200 spermatozoa are counted, the SE is $14(\sqrt{2} 200)$, and the $95 \% \mathrm{Cl}$ is $172-228(200 \pm 28)$. If 400 spermatozoa are counted, the SE is $20(\sqrt{ } 400)$ and the $95 \% \mathrm{Cl}$ is $360-440(400 \pm 40)$.

The sampling errors can be conveniently expressed as a percentage of the count $(100 \times(\sqrt{ } \mathrm{N} / \mathrm{N}))$. These are shown in Table 2.2 . 
Note: These values are only approximate, as confidence intervals are not always symmetrical around the estimate. The exact 95\% confidence intervals, based on the properties of the Poisson distribution, are $361-441$ for a count of $400,81.4-121$ for a count of $100,4.80-18.4$ for a count of $10,0.03-5.57$ for a count of 1 , and $0.00-3.70$ for a count of 0 .

Table 2.2 Rounded sampling errors (\%) according to total number of spermatozoa counted

\begin{tabular}{|c|c|}
\hline Total (N) & $\begin{array}{c}\text { Sampling } \\
\text { error (\%) }\end{array}$ \\
\hline 1 & 100 \\
\hline 2 & 70.7 \\
\hline 3 & 57.7 \\
\hline 4 & 50 \\
\hline 5 & 44.7 \\
\hline 6 & 40.8 \\
\hline 7 & 37.8 \\
\hline 8 & 35.4 \\
\hline 9 & 33.3 \\
\hline 10 & 31.6 \\
\hline 15 & 25.8 \\
\hline 20 & 22.4 \\
\hline \multicolumn{2}{|r|}{} \\
\hline
\end{tabular}

\begin{tabular}{|c|c|}
\hline Total (N) & $\begin{array}{c}\text { Sampling } \\
\text { error }(\%)\end{array}$ \\
\hline 25 & 20 \\
\hline 30 & 18.3 \\
\hline 35 & 16.9 \\
\hline 40 & 15.8 \\
\hline 45 & 14.9 \\
\hline 50 & 14.1 \\
\hline 55 & 13.5 \\
\hline 60 & 12.9 \\
\hline 65 & 12.4 \\
\hline 70 & 12 \\
\hline 75 & 11.5 \\
\hline 80 & 11.2 \\
\hline
\end{tabular}

\begin{tabular}{|c|c|}
\hline Total (N) & $\begin{array}{c}\text { Sampling } \\
\text { error (\%) }\end{array}$ \\
\hline 85 & 10.8 \\
\hline 90 & 10.5 \\
\hline 95 & 10.3 \\
\hline 100 & 10 \\
\hline 150 & 8.2 \\
\hline 200 & 7.1 \\
\hline 250 & 6.3 \\
\hline 300 & 5.8 \\
\hline 350 & 5.3 \\
\hline 400 & 5 \\
\hline 450 & 4.7 \\
\hline 500 & 4.5 \\
\hline
\end{tabular}

Comment 1: Counting too few spermatozoa will produce an uncertain result (see Appendix 7, section A7.1), which may have consequences for diagnosis and therapy (see Appendix 7, section A7.2). This may be unavoidable when spermatozoa are taken for therapeutic purposes and sperm numbers are low (see Section 5.1).

Comment 2: When semen volume is small and fewer spermatozoa are counted than recommended, the precision of the values obtained will be significantly reduced. If fewer than 200 spermatozoa are counted per replicate, report the sampling error as given in Table 2.2.

\subsection{Routine counting procedure}

The dilutions $1+4(1: 5)$ and $1+19(1: 20)$ are appropriate for a range of sperm concentrations, yielding about 200 spermatozoa in one or all of the haemocytometer grid numbers 4, 5 and 6 (see Table 2.3 and Box 2.8). 
Box 2.8 Achieving 200 spermatozoa per replicate in the central three grids of the improved Neubauer chamber

If there are 100 spermatozoa per high-power field (HPF) of $4 \mathrm{nl}$ (see Box 2.9) in the initial wet preparation, there are theoretically 25 per nl $(25000$ per $\mu$ lor 25000000 per $\mathrm{ml}$ ). As the central grid (number 5 ) of the improved Neubauer chamber holds $100 \mathrm{nl}$, there would be 2500 spermatozoa within it. Diluting the sample $1+4$ (1:5) would reduce the background and the sperm number to about 500 per grid, which is sufficient for an acceptably low sampling error.

If there are 10 spermatozoa per HPF of the wet preparation, there would be 2.5 per $\mathrm{nl}$ and 250 per central grid. Diluting the sample $1+1(1: 2)$ as suggested would reduce the background and the sperm number to about 125 per grid; this would give 375 in the three grids numbered 4, 5 and 6-again, this is sufficient for an acceptably low sampling error.

Note: These calculated concentrations can only be rough estimates because so few spermatozoa are counted and volumes may not be accurate. The concentrations estimated from the undiluted preparations can be between $30 \%$ and $130 \%$ of the concentrations derived from diluted samples in counting chambers.

\subsubsection{Determining the required dilution}

The dilution of semen required to allow sperm number to be measured accurately is assessed from an undiluted semen preparation. This is usually the wet preparation (see Section 2.4.2) used for evaluation of motility.

- Examine one of the wet preparations, made as described in Section 2.4.2, to estimate the number of spermatozoa per HPF ( $\times 200$ or $\times 400)$.

- One HPF is equivalent to approximately $16 \mathrm{nl}($ at $\times 200$ ) or $4 \mathrm{nl}$ (at $\times 400$ ) (see Box 2.9).

- If spermatozoa are observed, count them, determine the necessary dilution from Table 2.3, and proceed as in Section 2.8.2.

- If no spermatozoa are observed, examine the replicate wet preparation. If no spermatozoa are found in the second preparation, proceed as in Section 2.9.

Box 2.9 Volume observed per high-power field of a $20-\mu \mathrm{m}$-deep wet preparation

The volume of semen observed in each microscopic field depends on the area of the field $\left(\pi r^{2}\right.$, where $\pi$ is approximately 3.142 and $r$ is the radius of the microscopic field) and the depth of the chamber $(20.7 \mu \mathrm{m}$ for the wet preparation). The diameter of the microscopic field can be measured with a stage micrometer or can be estimated by dividing the diameter of the aperture of the ocular lens by the magnification of the objective lens.

With a $\times 40$ objective and a $\times 10$ ocular of aperture $20 \mathrm{~mm}$, the microscope field has a diameter of approximately $500 \mu \mathrm{m}(20 \mathrm{~mm} / 40)$. In this case, $r=250 \mu \mathrm{m}$, $r^{2}=62500 \mu \mathrm{m}^{2}, \pi r^{2}=196375 \mu \mathrm{m}^{2}$ and the volume is $4064962 \mu \mathrm{m}^{3}$ or about $4 \mathrm{nl}$. With a $\times 20$ objective and a $\times 10$ ocular of aperture $20 \mathrm{~mm}$, the microscope field has a diameter of approximately $1000 \mu \mathrm{m}(20 \mathrm{~mm} / 20)$. In this case, $r=500 \mu \mathrm{m}$, $r^{2}=250000 \mu \mathrm{m}^{2}, \pi r^{2}=785500 \mu \mathrm{m}^{2}$ and the volume is $16259850 \mu \mathrm{m}^{3}$ or about $16 \mathrm{nl}$. 
Table 2.3 Semen dilutions required, how to make them, which chambers to use and potential areas to assess

\begin{tabular}{|c|c|c|c|c|c|c|}
\hline $\begin{array}{c}\text { Spermatozoa } \\
\text { per } \times 400 \text { field }\end{array}$ & $\begin{array}{c}\text { Spermatozoa } \\
\text { per } \times 200 \text { field }\end{array}$ & $\begin{array}{c}\text { Dilution } \\
\text { required }\end{array}$ & $\begin{array}{c}\text { Semen } \\
(\mu \mathrm{l})\end{array}$ & $\begin{array}{c}\text { Fixative } \\
(\mu \mathrm{l})\end{array}$ & Chamber & $\begin{array}{c}\text { Area to be } \\
\text { assessed }\end{array}$ \\
\hline$>101$ & $>404$ & $1: 20(1+19)$ & 50 & 950 & $\begin{array}{c}\text { Improved } \\
\text { Neubauer }\end{array}$ & Grids 5, 4, 6 \\
\hline $16-100$ & $64-400$ & $1: 5(1+4)$ & 50 & 200 & $\begin{array}{c}\text { Improved } \\
\text { Neubauer }\end{array}$ & Grids 5, 4, 6 \\
\hline $2-15$ & $8-60$ & $1: 2(1+1)$ & 50 & 50 & $\begin{array}{c}\text { Improved } \\
\text { Neubauer }\end{array}$ & Grids 5, 4, 6 \\
\hline$<2$ & $<8$ & $1: 2(1+1)$ & 50 & 50 & $\begin{array}{c}\text { Improved } \\
\text { Neubauer } \\
\text { or } \\
\text { large-volume }\end{array}$ & $\begin{array}{c}\text { All } 9 \text { grids } \\
\text { or } \\
\text { Entire slide }\end{array}$ \\
\hline
\end{tabular}

Note 1: White-blood-cell pipettes and automatic pipettes that rely on air displacement are not accurate enough for making volumetric dilutions of viscous semen; use positive-displacement pipettes.

Note 2: For diagnostic purposes, semen samples for analysis should be not less than $50 \mu \mathrm{l}$ in volume, to avoid pipetting errors associated with small volumes.

Note 3: If there are too few spermatozoa per field of view at the recommended dilution, prepare another, lower, dilution. If there are too many overlapping spermatozoa per field of view at the recommended dilution, prepare another, higher, dilution.

Note 4: If a $1+19(1: 20)$ dilution is inadequate, use $1+49(1: 50)$.

Comment 1: If the number of spermatozoa in the initial wet preparation is low ( $<4$ per $\times 400 \mathrm{HPF}$ : approximately $1 \times 10^{6} / \mathrm{ml}$ ) an accurate sperm number may not be required (see Section 2.10).

Comment 2: For accurate assessment of low sperm concentrations $(<2$ per $\times 400$ HPF : < approximately $0.5 \times 10^{6} / \mathrm{ml}$ ), it is recommended to use all nine grids of the improved Neubauer chamber (see Section 2.11.1) or a large-volume disposable chamber with fluorescence detection (see Section 2.11.2).

2.8.2 Preparing the dilutions and loading the haemocytometer chambers

- Make the haemocytometer surface slightly damp by breathing on it.

- Secure the coverslip on the counting chambers by pressing it firmly onto the chamber pillars. Iridescence (multiple Newton's rings) between the two glass surfaces confirms the correct positioning of the coverslip. The more lines there are, the better the fit; only one or two lines may indicate problems with variation in chamber depth. 
- Use a positive-displacement pipette to dispense the appropriate amount of fixative (see Table 2.3) into two dilution vials.

- Mix the semen sample well (see Box 2.3).

- Aspirate the appropriate volume of semen immediately after mixing, allowing no time for the spermatozoa to settle out of suspension (see Table 2.3).

- Wipe the semen off the outside of the pipette tip, taking care not to touch the opening of the tip.

- Dispense the semen into the fixative and rinse the pipette tip by aspirating and expressing the fixative.

- Mix the semen sample well again, and prepare the replicate dilution following the steps above.

- Mix the first dilution thoroughly by vortexing for 10 seconds at maximum speed. Immediately remove approximately $10 \mu \mathrm{l}$ of fixed suspension, to avoid settling of the spermatozoa.

- Touch the pipette tip carefully against the lower edge of one of the chambers at the V-shaped groove.

- Depress the plunger of the pipette slowly, allowing the chamber to fill by capillary action. The coverslip should not be moved during filling, and the chamber should not be overfilled (when the coverslip may be seen to move) or underfilled (when air occupies some of the chamber area).

- Mix the second dilution, as above, and immediately remove a second $10-\mu 1$ aliquot. Load the second chamber of the haemocytometer following the steps above.

- Store the haemocytometer horizontally for at least 4 minutes at room temperature in a humid chamber (e.g. on water-saturated filter paper in a covered Petri dish) to prevent drying out. The immobilized cells will sediment onto the grid during this time.

Note 1: Some chambers are constructed with ground-glass pillars; in these, Newton's rings will not appear. Apply about $1.5 \mu$ of water to each ground-glass pillar to hold the coverslip in place (Brazil et al., 2004a), taking care not to introduce water into the counting area.

Note 2: The use of haemocytometer clamps to hold the coverslip in place will ensure a constant depth (Christensen et al., 2005).

Note 3: In very viscous samples, semen can aggregate within the dilution fluid if mixing is delayed by 5-10 seconds. In these cases, vortex the diluted sample for 10 seconds immediately after adding the semen to the fixative. 


\subsubsection{Assessing sperm numbers in the counting chambers}

Sperm number should be assessed in both chambers of the haemocytometer. If the two values agree sufficiently, the aliquots taken can be considered representative of the sample (see Section 2.4.1).

- Examine the haemocytometer with phase-contrast optics at $\times 200$ or $\times 400$ magnification.

- Count at least 200 spermatozoa in each replicate, in order to achieve an acceptably low sampling error (see Box 2.7 and Table 2.2).

- First assess the central grid (number 5 in Fig. 2.7) of one side of the improved Neubauer chamber, row by row.

- Continue counting until at least 200 spermatozoa have been observed and a complete row (of five large squares) has been examined. Counting must be done by complete rows; do not stop in the middle of a row. If 200 spermatozoa are not observed in the five rows of the central grid, continue counting in the rows (of 4 large squares) of the two adjacent grids (nos 4 and 6 in Fig. 2.7).

- Make a note of the number of rows assessed to reach at least 200 spermatozoa. The same number of rows will be counted from the other chamber of the haemocytometer.

- Tally the number of spermatozoa and rows with the aid of a laboratory counter.

- Switch to the second chamber of the haemocytometer and perform the replicate count on the same number of rows (the same volume) as the first replicate, even if this yields fewer than 200 spermatozoa.

- Calculate the sum and difference of the two numbers.

- Determine the acceptability of the difference from Table 2.4 or Fig. A7.1, Appendix 7. (Each shows the maximum difference between the counts that is expected to occur in $95 \%$ of samples because of sampling error alone.)

- If the difference is acceptable, calculate the concentration (see Section 2.8.4). If the difference is too high, prepare two new dilutions as described in Section 2.8.2 and repeat replicate counts (see Box 2.10).

- Report the average sperm concentration to two significant figures.

- Calculate the total number of spermatozoa per ejaculate (see Section 2.8.7).

Note 1: If fewer than 200 spermatozoa are found in grids 4,5 and 6 , do not continue to count in grids $1,2,3,7,8$ or 9 , since the volume of each row in these grids differs from that of the rows in grids 4, 5 and 6 (see Section 2.7.2). In this case, prepare and assess two lower dilutions. If a $1+1(1: 2)$ dilution is necessary, proceed as in Section 2.11.

Note 2: Assessing the same chamber twice or assessing both chambers filled from a single dilution is not true replication, as this will not allow detection of errors of sampling, mixing and dilution. 
Table 2.4 Acceptable differences between two replicate counts for a given sum

\begin{tabular}{|c|c|}
\hline Sum & $\begin{array}{c}\text { Acceptable } \\
\text { Difference* }\end{array}$ \\
\hline $144-156$ & 24 \\
\hline $157-169$ & 25 \\
\hline $170-182$ & 26 \\
\hline $183-196$ & 27 \\
\hline $197-211$ & 28 \\
\hline $212-226$ & 29 \\
\hline $227-242$ & 30 \\
\hline $243-258$ & 31 \\
\hline $259-274$ & 32 \\
\hline $275-292$ & 33 \\
\hline $293-309$ & 34 \\
\hline $310-328$ & 35 \\
\hline
\end{tabular}

\begin{tabular}{|c|c|}
\hline Sum & $\begin{array}{c}\text { Acceptable } \\
\text { Difference* }\end{array}$ \\
\hline $329-346$ & 36 \\
\hline $347-366$ & 37 \\
\hline $367-385$ & 38 \\
\hline $386-406$ & 39 \\
\hline $407-426$ & 40 \\
\hline $427-448$ & 41 \\
\hline $449-470$ & 42 \\
\hline $471-492$ & 43 \\
\hline $493-515$ & 44 \\
\hline $516-538$ & 45 \\
\hline $539-562$ & 46 \\
\hline $563-587$ & 47 \\
\hline
\end{tabular}

*Based on the rounded $95 \%$ confidence interval.

\section{Box 2.10 Comparison of replicate counts}

The difference between independent counts is expected to be zero, with a standard error equal to the square root of the sum of the two counts. Thus (N1-N2)/ $(\sqrt{ }(\mathrm{N} 1+\mathrm{N} 2))$ should be $<1.96$ by chance alone for a $95 \%$ confidence limit.

If the difference between the counts is less than or equal to that indicated in Tables 2.4 or 2.5 for the given sum, the estimates are accepted and the concentration is calculated from their mean.

Larger differences suggest that miscounting has occurred, or there were errors of pipetting, or the cells were not well mixed, resulting in non-random distribution in the chamber or on the slide.

When the difference between the counts is greater than acceptable, discard the first two values, and prepare and assess two fresh dilutions of semen. (Do not count a third sample and take the mean of the three values, or take the mean of the two closest values.)

This applies to counts of spermatozoa and peroxidase-positive cells (see Section 2.18). For CD45-positive cells (see Section 3.2) and immature germ cells (see Section 2.19), the stained preparations should be reassessed.

With these $95 \% \mathrm{Cl}$ cut-off values, approximately $5 \%$ of replicates will be outside the limits by chance alone.

Note: On rare occasions, with inhomogeneous samples, even a third set of replicates may provide unacceptable differences. In this case, calculate the mean of all replicates and note this in the report. 
2.8.4 Calculation of the concentration of spermatozoa in semen

It is recommended to calculate and report on the concentration of spermatozoa in semen. Although concentration is not a specific measure of testicular function, it is related to fertilization and pregnancy rates.

The concentration of spermatozoa in semen is their number $(\mathrm{N})$ divided by the volume in which they were found, i.e. the volume of the total number ( $n$ ) of rows examined for the replicates ( $20 \mathrm{nl}$ each for grids 4,5 and 6 ), multiplied by the dilution factor. That is, $\mathrm{C}=(\mathrm{N} / \mathrm{n}) \times(1 / 20) \times$ dilution factor.

For $1+4(1: 5)$ dilutions, using grids 4,5 and 6 , the concentration $\mathrm{C}=(\mathrm{N} / \mathrm{n}) \times(1 / 20) \times 5$ spermatozoa per $\mathrm{nl}=(\mathrm{N} / \mathrm{n}) \times(1 / 4)$ spermatozoa $/ \mathrm{nl}$ (or $10^{6} \mathrm{per}$ $\mathrm{ml}$ of semen).

For $1+19(1: 20)$ dilutions, using grids 4,5 and 6 , the concentration $\mathrm{C}=(\mathrm{N} / \mathrm{n}) \times(1 / 20) \times 20$ spermatozoa per $\mathrm{nl}=(\mathrm{N} / \mathrm{n})$ spermatozoa $/ \mathrm{nl}$ (or $10^{6}$ per $\mathrm{ml}$ of semen).

For $1: 50(1+49)$ dilutions, using grids 4,5 and 6 , the concentration $\mathrm{C}=(\mathrm{N} / \mathrm{n}) \times(1 / 20) \times 50$ spermatozoa per $\mathrm{nl}=(\mathrm{N} / \mathrm{n}) \times 2.5$ spermatozoa $/ \mathrm{nl}$ (or $10^{6}$ per $\mathrm{ml}$ of semen).

\subsubsection{Worked examples}

Example 1. With a $1+19(1: 20)$ dilution, replicate 1 is found to contain 201 spermatozoa in seven rows, while replicate 2 contains 245 spermatozoa in seven rows. The sum of the values $(201+245)$ is 446 in 14 rows and the difference $(245-201)$ is 44. From Table 2.4 this is seen to exceed the difference expected by chance alone (41), so new replicate dilutions are made.

Example 2. With a $1+19(1: 20)$ dilution, replicate 1 is found to contain 220 spermatozoa in four rows, while replicate 2 contains 218 spermatozoa in four rows. The sum of the values $(220+218)$ is 438 in eight rows and the difference $(220-218)$ is 2 . From Table 2.4 this is seen to be less than that found by chance alone (41), so the values are accepted.

The concentration of the samples for a $1+19(1: 20)$ dilution is $C=(\mathrm{N} / \mathrm{n}) \times 1.0$ spermatozoa per nl, i.e. $(438 / 8) \times 1.0=54.75 \mathrm{spermatozoa} / \mathrm{nl}$, or $55 \times 10^{6}$ spermatozoa per $\mathrm{ml}$ of semen (to two signific ant figures).

Note: For $1+19(1: 20)$ dilutions and grids 4,5 and 6 , the concentration is easy to calculate. The total number of spermatozoa counted divided by the total number of rows assessed equals the sperm concentration in $10^{6} / \mathrm{ml}$. In the example above the calculation is $(220+218) /(4+4)=438 / 8=55 \times 10^{6}$ spermatozoa per $\mathrm{ml}$ of semen.

Example 3. With a $1+19(1: 20)$ dilution, replic ate 1 is found to contain 98 spermatozoa in 15 rows (grids 5, 4 and 6), while replicate 2 contains 114 spermatozoa in 15 rows (grids 5,4 and 6$)$. The sum of the values $(98+114)$ is 212 in 30 rows and the difference (114-98) is 16 . From Table 2.4 this is seen to be less than that found by chance alone (29), so the values are accepted. 
The concentration of the sample for a $1+19(1: 20)$ dilution is $C=(\mathrm{N} / \mathrm{n}) \times 1.0$ spermatozoa per $\mathrm{nl}$ or $(212 / 30) \times 1.0=7.07$ spermatozoa/nl, or $7.1 \times 10^{6}$ spermatozoa per $\mathrm{ml}$ of semen (to two significant figures). As fewer than 400 spermatozoa were counted, report the sampling error for 212 spermatozoa given in Table 2.2 (approximately $7 \%$ ).

Note: In this example, the sample has been overdiluted, since fewer than 200 spermatozoa were found in grids 5,4 and $6 ; a 1+4(1: 5)$ dilution would have been more appropriate.

Example 4. With a $1+4(1: 5)$ dilution, replicate 1 is found to contain 224 spermatozoa in four rows, while replicate 2 contains 268 spermatozoa in four rows. The sum of the values $(224+268)$ is 492 in eight rows and the difference $(268-224)$ is 44. From Table 2.4 this is seen to exceed the difference expected by chance alone (43), so new replicate dilutions are made.

Example 5. With a $1+4(1: 5)$ dilution, replicate 1 is found to contain 224 spermatozoa in eight rows, while replicate 2 contains 213 spermatozoa in eight rows. The sum of the values $(224+213)$ is 437 in 16 rows and the difference $(224-213)$ is 11 . From Table 2.4 this is seen to be less than that found by chance alone (41), so the values are accepted.

The concentration of the sample for a $1+4(1: 5)$ dilution is $C=(\mathrm{N} / \mathrm{n}) \times(1 / 4)$ spermatozoa per $\mathrm{nl}$ or $(437 / 16) / 4=6.825$ spermatozoa/nl, or $6.8 \times 10^{6}$ spermatozoa per $\mathrm{ml}$ of semen (to two significant figures).

Note: For $1+4(1: 5)$ dilutions the concentration is also simple to calculate but the total number of spermatozoa counted divided by the total number of rows assessed is further divided by 4 . In the example above the calculation is $((224+213) /$ $(8+8)) / 4=(437 / 16) / 4=27.3 / 4=6.8 \times 10^{6}$ spermatozoa per $\mathrm{ml}$ of semen.

\subsubsection{Lower reference limit for sperm concentration}

The lower reference limit for sperm concentration is $15 \times 10^{6}$ spermatozoa per $\mathrm{ml}$ (5th centile, $95 \% \mathrm{Cl} 12-16 \times 10^{6}$ ).

2.8.7 Calculation of the total number of spermatozoa in the ejaculate

It is recommended to calculate and report the total number of spermatozoa per ejaculate, as this parameter provides a measure of the capability of the testes to produce spermatozoa and the patency of the male tract. This is obtained by multiplying the sperm concentration by the volume of the whole ejaculate.

2.8.8 Lower reference limit for total sperm number

The lower reference limit for total sperm number is $39 \times 10^{6}$ spermatozoa per ejaculate (5th centile, $95 \% \mathrm{CI} 33-46 \times 10^{6}$ ). 


\subsection{Low sperm numbers: cryptozoospermia and suspected azoospermia}

If no spermatozoa are observed in the replicate wet preparations, azoospermia can be suspected. Although it has been suggested that the definition should change (Sharif, 2000; Ezeh \& Moore, 2001), azoospermia remains a description of the ejaculate rather than a statement of its origin or a basis for diagnosis and therapy. It is generally accepted that the term azoospermia can only be used if no spermatozoa are found in the sediment of a centrifuged sample (Eliasson, 1981).

However, it should be borne in mind that:

- whether or not spermatozoa are found in the pellet depends on the centrifugation time and speed (Lindsay et al., 1995; J affe et al., 1998) and on how much of the pellet is examined;

- centrifugation at $3000 \mathrm{~g}$ for 15 minutes does not pellet all spermatozoa from a sample (Corea et al., 2005); and

- after centrifugation, motility can be lost (Mortimer, 1994a) and concentration will be underestimated (Cooper et al., 2006).

The way these samples are handled depends on whether subjective data on the presence and motility of spermatozoa are sufficient (see Section 2.10) or accurate numbers of spermatozoa are required (see Section 2.11).

\subsection{When an accurate assessment of low sperm numbers is not required}

If the number of spermatozoa per HPF in the initial wet preparation is low (0 to 4 per $\times 400 \mathrm{HPF}$ or 0 to 16 per $\times 200 \mathrm{HPF}$ ), several options are available.

2.10.1 Taking no further action

If the number of spermatozoa per $\times 400 \mathrm{HPF}$ is $<4$ (i.e. $<$ approximately $1 \times 10^{6} / \mathrm{ml}$ ), it is sufficient for most clinical purposes to report the sperm concentration as $<2 \times 10^{6} / \mathrm{ml}$ (to take into account the high sampling error associated with low sperm numbers), with a note as to whether or not motile spermatozoa were seen.

2.10.2 Examination of centrifuged samples to detect spermatozoa

When no spermatozoa are observed in either wet preparation, the sample can be centrifuged to determine if any spermatozoa are present in a larger sample.

- Mix the semen sample well (see Box 2.3). If the sample is viscous, reduce the viscosity as described in Section 2.3.1.1.

- Remove a 1-ml aliquot of semen and centrifuge at $3000 \mathrm{~g}$ for 15 minutes.

- Decant most of the supernatant and resuspend the sperm pellet in the remaining approximately $50 \mu \mathrm{l}$ of seminal plasma. 
- Place one 10- $\mu \mathrm{l}$ aliquot of the pellet on each of two slides under $22 \mathrm{~mm} \times 22 \mathrm{~mm}$ coverslips. This will create two wet preparations approximately $20 \mu \mathrm{m}$ deep (see Box 2.4).

- Examine the slides with phase-contrast optics at $\times 200$ or $\times 250$ magnification.

- Scan the entire coverslip systematically field by field. Start in one corner and scan along the $x$-axis to the opposite side; then move one field along the $y$-axis and scan back along the entire width. Continue in this zig-zag fashion to make a complete and systematic search of the entire aliquot (see Fig. 2.9). Keep observing the slide while changing fields.

- With a $\times 20$ objective and a $\times 10$ ocular of $20 \mathrm{~mm}$ aperture, the microscope field has a diameter of approximately $1000 \mu \mathrm{m}$ (see B ox 2.9). There will thus be approximately 484 fields $(22 \times 22)$ per $22 \mathrm{~mm} \times 22 \mathrm{~mm}$ coverslip to be examined.

- The presence of spermatozoa in either replic ate indicates cryptozoospermia.

- The absence of spermatozoa from both replicates suggests azoospermia.

Note 1: Many bench-top centrifuges that take $15-\mathrm{ml}$ tubes will not reach $3000 \mathrm{~g}$ : use a higher-speed centrifuge that takes 1.5-2.0-ml tubes. Make sure the semen sample is well mixed before taking the aliquot.

Note 2: Scanning the slides can take up to 10 minutes, as the sample will have a high background.

Note 3: When centrifuging samples for assisted reproduction, the whole semen sample and most of the pellet (e.g. four $10-\mu$ l aliquots of pellet) may need to be analysed to find live spermatozoa.

Comment 1: The absence of motile spermatozoa from the aliquot examined does not necessarily mean that they are absent from the rest of the sample.

Comment 2: Because centrifugation does not pellet all spermatozoa, this method cannot be used to determine total sperm number. For quantification, see Sections 2.11.1 or 2.11.2.

2.10.3 Examination of non-centrifuged samples to detect motile spermatozoa

When motile spermatozoa are sought (e.g. in a post-vasectomy semen sample), diluting the specimen in fixative or high-speed centrifugation of spermatozoa must be avoided. In this case, only an aliquot of the undiluted sample can be assessed.

- Mix the semen sample well (see Box 2.3).

- Remove a 40- $\mu$ l aliquot of semen and place under a $24 \mathrm{~mm} \times 50 \mathrm{~mm}$ coverslip. This will create a wet preparation $33 \mu \mathrm{m}$ deep (see Box 2.4).

- Examine the slide with phase-contrast optics at $\times 200$ or $\times 250$ magnification.

- Scan the entire coverslip systematically field by field. Start in one corner and scan along the $x$-axis to the opposite side; then move one field along the $y$-axis 
and scan back along the entire width. Continue in this zig-zag fashion to make a complete and systematic search of the entire aliquot (see Fig. 2.9). Keep observing the slide while changing fields.

- With a $\times 20$ objective and $\times 10$ ocular of $20 \mathrm{~mm}$ aperture, the microscope field has a diameter of approximately $1000 \mu \mathrm{m}$ (see Box 2.9). There will thus be approximately 1200 fields $(24 \times 50)$ per $24 \mathrm{~mm} \times 50 \mathrm{~mm}$ coverslip to be examined.

Note: This procedure can take up to 10 minutes, as the sample will have a high background.

Fig. 2.9 Scanning the entire coverslip for the presence of motile spermatozoa

This involves assessing approximately 1200 high-power fields at $\times 200$ magnification for a $24 \mathrm{~mm} \times 50 \mathrm{~mm}$ coverslip, and approximately 484 high-power fields at $\times 200$ magnification for a $22 \mathrm{~mm} \times 22 \mathrm{~mm}$ coverslip.

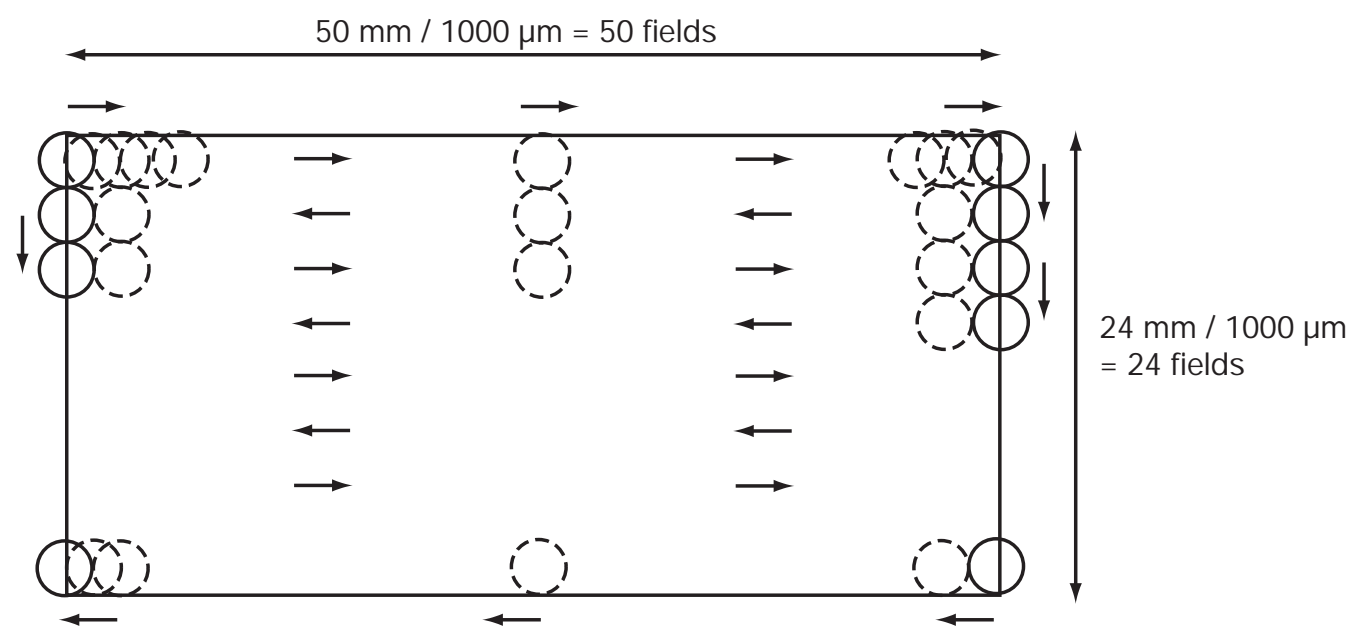

Total 1200 fields

Comment: The absence of motile spermatozoa from the aliquot examined does not necessarily mean that they are absent from the rest of the sample. 


\subsection{When an accurate assessment of low sperm numbers is required}

This section describes methods for determining low sperm concentrations that avoid centrifugation. The alternative to pelleting the spermatozoa is to use a low dilution of semen and to examine larger volumes.

A precision of $20 \%$ is considered acceptable when dealing with lower limits of quantification (LLQ) (Shah et al., 2000). Examining the entire central grid (number 5 in Fig. 2.7) of the improved Neubauer chamber, when filled with $1+1$ (1:2) diluted semen, can theoretically detect a concentration of 250000 spermatozoa per $\mathrm{ml}$ with a sampling error of $20 \%$. When all nine grids are examined, a sperm concentration as low as 27800 per $\mathrm{ml}$ can be estimated. Large-volume disposable chambers holding $25 \mu \mathrm{l}$ can be used to measure a concentration of 1000 spermatozoa per $\mathrm{ml}$ with the same sampling error (Cooper et al., 2006). For semen diluted $1+1(1: 2)$, as recommended here, these values correspond to sperm concentrations in undiluted semen of 500000 per $\mathrm{ml}, 55600 \mathrm{per} \mathrm{ml}$ and 2000 per ml, respectively. However, semen samples diluted so little can present a large amount of background. Scanning large chambers can take 10-20 minutes, but rapid detection of spermatozoa can be facilitated by use of a fluorescent dye (see Section 2.11.2).

2.11.1 Assessing low sperm numbers in the entire improved Neubauer chamber (phase-contrast microscopy)

To reduce sampling errors, a critical number of spermatozoa (preferably a total of at least 400 from replicate counts of approximately 200 ) have to be counted (see Box 2.7 and Table 2.2).

- Mix the semen sample well (see Box 2.3).

- Remove an aliquot of semen and dilute $1+1(1: 2)$ with fixative (see Section 2.7.5), taking the precautions given in Section 2.8.2.

- The dilution $1+1$ (1:2) for samples with fewer than two spermatozoa per HPF in the initial wet preparation (Table 2.3 ) is appropriate for a range of sperm concentrations, yielding about 200 spermatozoa in the haemocytometer (see Box 2.11). Between one and nine grids will need to be assessed.

Box 2.11 Achieving 200 spermatozoa per replicate in all nine grids of the improved Neubauer chamber

If there are 2 spermatozoa per HPF of $4 \mathrm{nl}$ in the initial wet preparation, there are theoretically 0.5 spermatozoa per $\mathrm{nl}(500$ spermatozoa per $\mu$ l or 500000 spermatozoa per $\mathrm{ml}$ ).

As all 9 grids of the improved Neubauer chamber together hold $900 \mathrm{nl}$, there would be 450 spermatozoa in them. Diluting the sample $1+1(1: 2)$, as suggested, would reduce the background and the sperm number to 225 per chamber, sufficient for an acceptably low sampling error. 
Note: This value can only be a rough estimate because so few spermatozoa are counted and volumes may be inaccurate.

\subsubsection{Procedure}

1. Dilute two aliquots of the semen sample $1+1(1: 2)$ with fixative, as above.

2. Fill each chamber of the haemocytometer with the replicate dilutions, one replicate per chamber.

3. Store the haemocytometer horizontally for at least 4 minutes at room temperature in a humid chamber (e.g. on water-saturated filter paper in a covered Petri dish) to prevent drying out. The immobilized cells will sediment onto the grid during this time.

4. Examine the haemocytometer with phase-contrast optics at $\times 200$ or $\times 400$ magnification.

5. Count at least 200 spermatozoa in each replicate, in order to achieve an acceptably low sampling error (see Box 2.7 and Table 2.2).

6. Examine one chamber grid by grid, and continue counting until at least 200 spermatozoa have been observed and a complete grid has been examined. Counting must be done by complete grids; do not stop in the middle of a grid.

7. Make a note of the number of grids assessed to reach at least 200 spermatozoa. The same number of grids will be counted from the other chamber of the haemocytometer.

8. Tally the number of spermatozoa and grids with the aid of a laboratory counter.

9. Switch to the second chamber of the haemocytometer and perform the replicate count on the same number of grids (the same volume) as the first replicate, even if this yields fewer than 200 spermatozoa.

10. Calculate the sum and difference of the two numbers.

11. Determine the acceptability of the difference from Table 2.5 (which extends Table 2.4 to lower sperm numbers) or Fig. A7.1, Appendix 7. (Each shows the maximum difference between two counts that is expected to occur in $95 \%$ of samples because of sampling error alone).

12. If the difference is acceptable, calculate the concentration (see Section 2.11.1.2). If the difference is too high, make two new preparations as described above and repeat replicate counts (see Box 2.10).

13. Report the average sperm concentration to two significant figures.

14. Calculate the total number of spermatozoa per ejaculate (see Section 2.11.1.5). 
Table 2.5 Acceptable differences between two counts for a given sum: low concentrations

\begin{tabular}{|c|c|}
\hline Sum & $\begin{array}{c}\text { Acceptable } \\
\text { difference* }\end{array}$ \\
\hline $35-40$ & 12 \\
\hline $41-47$ & 13 \\
\hline $48-54$ & 14 \\
\hline $55-62$ & 15 \\
\hline $63-70$ & 16 \\
\hline $71-79$ & 17 \\
\hline $80-89$ & 18 \\
\hline $90-98$ & 19 \\
\hline $99-109$ & 20 \\
\hline $110-120$ & 21 \\
\hline $121-131$ & 22 \\
\hline $132-143$ & 23 \\
\hline
\end{tabular}

\begin{tabular}{|c|c|}
\hline Sum & $\begin{array}{c}\text { Acceptable } \\
\text { difference* }\end{array}$ \\
\hline $144-156$ & 24 \\
\hline $157-169$ & 25 \\
\hline $170-182$ & 26 \\
\hline $183-196$ & 27 \\
\hline $197-211$ & 28 \\
\hline $212-226$ & 29 \\
\hline $227-242$ & 30 \\
\hline $243-258$ & 31 \\
\hline $259-274$ & 32 \\
\hline $275-292$ & 33 \\
\hline $293-309$ & 34 \\
\hline $310-328$ & 35 \\
\hline
\end{tabular}

\begin{tabular}{|c|c|}
\hline Sum & $\begin{array}{c}\text { Acceptable } \\
\text { difference* }\end{array}$ \\
\hline $329-346$ & 36 \\
\hline $347-366$ & 37 \\
\hline $367-385$ & 38 \\
\hline $386-406$ & 39 \\
\hline $407-426$ & 40 \\
\hline $427-448$ & 41 \\
\hline $449-470$ & 42 \\
\hline $471-492$ & 43 \\
\hline $493-515$ & 44 \\
\hline $516-538$ & 45 \\
\hline $539-562$ & 46 \\
\hline $563-587$ & 47 \\
\hline
\end{tabular}

*Based on the rounded $95 \%$ confidence interval.

2.11.1.2 Calculation of low concentrations of spermatozoa in semen

The concentration of spermatozoa in semen is their number $(N)$ divided by the volume in which they were found, i.e. the volume of the total number $(n)$ of grids examined for the replicates (where the volume of a grid is $100 \mathrm{nl}$ ), multiplied by the dilution factor. That is, $C=(\mathrm{N} / \mathrm{n}) \times(1 / 100) \times$ dilution factor.

For a $1+1(1: 2)$ dilution, the concentration $C=(N / n) \times(1 / 100) \times 2$ spermatozoa per $\mathrm{nl}=(\mathrm{N} / \mathrm{n}) \times(1 / 50)$ spermatozoa $/ \mathrm{nl}$.

When all nine grids are assessed in each chamber of the haemocytometer, the total number of spermatozoa is divided by the total volume of both chambers $(1.8 \mu \mathrm{l})$, and multiplied by the dilution factor (2), to obtain the concentration in spermatozoa per $\mu \mathrm{l}$ (thousands per $\mathrm{ml}$ of semen).

2.11.1.3 Sensitivity of the method

If there are fewer than 200 spermatozoa in each chamber, the sampling error will exceed $5 \%$. When fewer than 400 spermatozoa are found in both chambers, report the sampling error for the number of cells counted (see Table 2.2).

If fewer than 25 spermatozoa are counted in each chamber, the concentration will be $<56000$ spermatozoa per $\mathrm{ml}$; this is the lower limit of quantification for a sampling error of $20 \%$ when all nine grids of the improved Neubauer chamber are assessed and a $1+1$ (1:2) dilution is used (Cooper et al., 2006). Report the number of spermatozoa observed with the comment "Too few spermatozoa counted for accurate determination of concentration $(<56000 / \mathrm{ml})$ ". 
Comment: The absence of spermatozoa from the aliquot examined does not necessarily mean that they are absent from the rest of the sample.

\subsubsection{Worked examples}

Example 1. With a $1+1(1: 2)$ dilution, replicate 1 is found to contain 200 spermatozoa in two grids, while replicate 2 contains 250 spermatozoa in two grids. The sum of the values $(200+250)$ is 450 in four grids and the difference $(250-200)$ is 50. From Table 2.5 this is seen to exceed the difference expected by chance alone (42), so the results are discarded and two new replic ate dilutions are made.

Example 2. With a $1+1(1: 2)$ dilution, replicate 1 is found to contain 210 spermatozoa in three grids, while replicate 2 contains 200 spermatozoa in three grids. The sum of the values $(210+200)$ is 410 in six grids and the difference $(210-200)$ is 10 . From Table 2.5 this is seen to be less than that found by chance alone (40), so the values are accepted.

The concentration of spermatozoa in the sample for a $1+1(1: 2)$ dilution is $\mathrm{C}=$ $(\mathrm{N} / \mathrm{n}) \times(1 / 50)$ spermatozoa per $\mathrm{nl}$ or $(410 / 6) / 50=1.37$ spermatozoa $/ \mathrm{nl}$, or $1.4 \times 10^{6}$ spermatozoa per $\mathrm{ml}$ of semen (to two significant figures).

Example 3 . With a $1+1(1: 2)$ dilution, replicate 1 is found to contain 120 spermatozoa in all nine grids, while replicate 2 contains 140 spermatozoa in all nine grids. The sum of the values $(120+140)$ is 260 in 18 grids and the difference $(140-120)$ is 20. From Table 2.5 this is seen to be less than that found by chance alone (32), so the values are accepted.

When all nine grids are assessed in each chamber (a total of $1.8 \mu \mathrm{l}$ ), the concentration of spermatozoa in the sample for a $1+1(1: 2)$ dilution is $C=(N / 1.8) \times 2$ spermatozoa per $\mu \mathrm{l}=(260 / 1.8) \times 2=288.8 \mathrm{spermatozoa} / \mu \mathrm{l}$, or $290 \times 10^{3}$ spermatozoa per $\mathrm{ml}$ of semen (to two significant figures). As fewer than 400 spermatozoa were counted, report the sampling error for 260 spermatozoa as given in Table 2.2 (approximately 6\%).

Example 4. With a $1+1(1: 2)$ dilution, replicate 1 was found to contain 10 spermatozoa in all nine grids, while replic ate 2 contained 8 spermatozoa in all nine grids. As fewer than 25 spermatozoa were counted, the concentration is $<56000 /$ $\mathrm{ml}$; report that "18 spermatozoa were seen in the replicates, too few for accurate determination of concentration $(<56000 / \mathrm{ml})$ ".

Example 5. With a $1+1(1: 2)$ dilution, no spermatozoa are found in either replicate. As fewer than 25 spermatozoa were counted, the concentration is $<56000 /$ $\mathrm{ml}$; report that "No spermatozoa were seen in the replicates, too few for accurate determination of concentration $(<56000 / \mathrm{ml})$ ".

\subsubsection{Calculation of the total number of spermatozoa in the ejaculate}

It is recommended to calculate and report the total sperm number per ejaculate, as this parameter provides a measure of the capability of the testes to produce spermatozoa and the patency of the male tract. This is obtained by multiplying the sperm concentration by the volume of the whole ejaculate. 
2.11.2 Assessing low sperm numbers in large-volume disposable slides (fluorescence microscopy)

The use of large-volume, 100- $\mu \mathrm{m}$-deep chambers can increase the sensitivity of the concentration assessment (Cooper et al., 2006). The large-volume slide has two $100-\mu \mathrm{m}$ - deep chambers, each holding $25 \mu \mathrm{l}$. To reduce sampling errors, a critical number of spermatozoa (preferably a total of at least 400 from replicate counts of approximately 200) have to be counted (see Box 2.7 and Table 2.2).

- Mix the semen sample well (see Box 2.3).

- Remove an aliquot of semen and dilute $1+1(1: 2)$ with fixative (see Section 2.7.5) containing Hoechst 33342 bisbenzimide fluorochrome ( $1 \mathrm{mg} / \mathrm{l})$, taking the precautions given in Section 2.8.2.

The dilution $1+1$ (1:2) for samples with fewer than 2 spermatozoa at the initial evaluation (Table 2.3) is appropriate for a range of sperm concentrations, yielding about 200 spermatozoa within the entire chamber (see Box 2.12).

\section{Box 2.12 Achieving 200 spermatozoa per replicate in a $100-\mu \mathrm{m}$-deep, large-vol-} ume disposable chamber

If there is only 1 spermatozoon per HPF of $4 \mathrm{nl}$ in the initial wet preparation, there are theoretically 0.25 spermatozoa per $\mathrm{nl}(250$ per $\mu \mathrm{l}$ or 250000 per $\mathrm{ml}$ ).

The large-volume chamber holds $25 \mu \mathrm{l}$, so there would be 6250 spermatozoa within it. Diluting the sample $1+1(1: 2)$ as suggested would reduce the background and the sperm number to 3125 per chamber, sufficient for an acceptably low sampling error.

Note: This value can only be a rough estimate because so few spermatozoa are counted and the volumes may be inaccurate.

\subsubsection{Procedure}

1. Dilute two aliquots of the semen sample $1+1(1: 2)$ with fixative, as above.

2. Fill each chamber of the slide with $25 \mu$ l of the replicate dilutions, one replicate per chamber.

3. Store the slide horizontally for 10-15 minutes in the dark at room temperature in a humid chamber (e.g. on water-saturated filter paper in a covered Petri dish) to prevent drying out. The dye will bind to the sperm heads and the immobilized cells will settle on the chamber floor during this time.

4. Examine the slide with fluorescence optics using a relevant dichroic mirror and barrier filter at $\times 250$ magnification.

5. Count at least 200 spermatozoa in each replicate, in order to achieve an acceptably low sampling error (see Box 2.7 and Table 2.2).

6. Examine one chamber systematically field by field. Start in one corner and scan along the $x$-axis to the opposite side; then move one field along the $y$-axis 
and scan back along the entire width. Continue in this zig-zag fashion (see Fig. 2.9). Keep observing the slide while changing fields. Continue counting until at least 200 spermatozoa have been observed.

7. Make a note of the number of fields assessed to reach at least 200 spermatozoa. The same number of fields will be counted from the other chamber.

8. Tally the number of spermatozoa and fields with the aid of a laboratory counter.

9. Switch to the second chamber and perform the replicate count on the same number of fields (the same volume) as the first replicate, even if this yields fewer than 200 spermatozoa.

10. Calculate the sum and difference of the two numbers.

11. Determine the acceptability of the difference from Table 2.5 (which extends Table 2.4 to lower sperm numbers) or Fig. A7.1, Appendix 7. (Each shows the maximum difference between two counts that is expected to occur in $95 \%$ of samples because of sampling error alone).

12. If the difference is acceptable, calculate the concentration (see Section 2.11.2.2). If the difference is too high, make two new preparations and repeat the assessment (see Box 2.10).

13. Report the average sperm concentration to two significant figures.

14. Calculate the total number of spermatozoa per ejaculate (see Section 2.11.2.5).

Note 1: Spermatozoa appear as bright fluorescent points (condensed nuclei) unlike leukocytes and non-sperm cells, which have a more diffuse fluorescence (indicating their larger nuclei) (Zinaman et al., 1996).

Note 2: If uncertain about the source of a fluorescent signal, switch to phase-contrast optics where the sperm tail can be seen.

\subsubsection{Calculation of low concentrations of spermatozoa in semen}

The concentration of spermatozoa in semen is their number ( $N$ ) divided by the volume of the total number $(n)$ of microscopic fields examined (where the volume (v) of a field is calculated as in Box 2.13), multiplied by the dilution. That is, $\mathrm{C}=(\mathrm{N} / \mathrm{n}) \times(1 / \mathrm{v}) \times$ dilution factor.

At $\times 250$ magnification, the field volume is $80 \mathrm{nl}$ (see Box 2.13), and for a $1+1$ $(1: 2)$ dilution, the concentration is $C=(\mathrm{N} / \mathrm{n}) \times(1 / 80) \times 2$ spermatozoa per $\mathrm{nl}=$ $(\mathrm{N} / \mathrm{n}) \times(1 / 40)$ spermatozoa/nl (106 spermatozoa per $\mathrm{ml}$ of semen).

At $\times 400$ magnification, the field volume is $20 \mathrm{nl}$ (see Box 2.13), and for a $1+1$ $(1: 2)$ dilution, the concentration is $C=(N / n) \times(1 / 20) \times 2$ spermatozoa per $\mathrm{nl}=$ $(\mathrm{N} / \mathrm{n}) \times(1 / 10)$ spermatozoa/nl (106 spermatozoa per $\mathrm{ml}$ of semen).

When the entire area of both chambers has been assessed, the total number of spermatozoa is divided by the total volume of both chambers $(50 \mu \mathrm{l})$, multiplied by the dilution factor (2), to obtain the concentration in spermatozoa/ $\mu$ l (thousands per $\mathrm{ml}$ of semen). 
Box 2.13 Volume observed per high-power field in a $100-\mu \mathrm{m}$-deep, large-volume disposable chamber

The volume of semen in each microscopic field depends on the area of the field $\left(\pi r^{2}\right.$, where $\pi$ is approximately 3.142 and $r$ is the radius of the microscopic field) and the depth of the chamber (here $100 \mu \mathrm{m}$ ).

The diameter of the microscopic field can be measured with a stage micrometer or can be estimated by dividing the diameter of the aperture of the ocular lens by the magnification of the objective lens.

With a $\times 40$ objective and a $\times 10$ ocular of aperture $20 \mathrm{~mm}$, the microscope field has a diameter of approximately $500 \mu \mathrm{m}(20 \mathrm{~mm} / 40)$. In this case, $r=250 \mu \mathrm{m}, \mathrm{r}^{2}=$ $62500 \mu \mathrm{m}^{2}, \pi \mathrm{r}^{2}=196375 \mu \mathrm{m}^{2}$ and the volume is $19637500 \mu \mathrm{m}^{3}$ or about $20 \mathrm{nl}$.

With a $\times 25$ objective and a $\times 10$ ocular of aperture $25 \mathrm{~mm}$, the microscope field has a diameter of approximately $1000 \mu \mathrm{m}(25 \mathrm{~mm} / 25)$. In this case, $r=500 \mu \mathrm{m}, r^{2}=$ $250000 \mu \mathrm{m}^{2}, \pi \mathrm{r}^{2}=785500 \mu \mathrm{m}^{2}$ and the volume is $78550000 \mu \mathrm{m}^{3}$ or about $80 \mathrm{nl}$.

\subsubsection{Sensitivity of the method}

If there are fewer than 200 spermatozoa in each chamber, the sampling error will exceed $5 \%$. When fewer than 400 spermatozoa are found in both replicates, report the sampling error for the number of cells counted (see Table 2.2).

If fewer than 25 spermatozoa are counted in each chamber, the concentration will be $<2000$ spermatozoa/ml (this is the lower limit of quantification for a sampling error of $20 \%$ when the entire chamber $(25 \mu \mathrm{l})$ is assessed and a $1+1(1: 2)$ dilution is used) (Cooper et al., 2006). Report the number of spermatozoa observed with the comment "Too few spermatozoa counted for accurate determination of concentration $(<2000 / \mathrm{ml})$ ".

Comment: The absence of spermatozoa from the aliquot examined does not necessarily mean that they are absent from the rest of the sample.

\subsubsection{Worked examples}

Example 1 . With a $1+1(1: 2)$ dilution, replic ate 1 is found to contain 210 spermatozoa in 300 fields, while replicate 2 contains 300 spermatozoa in 300 fields. The sum of the values $(210+300)$ is 510 in 600 fields and the difference $(300-210)$ is 90. From Table 2.5 this is seen to exceed the difference expected by chance alone (44), so the results are discarded and two new replicate dilutions are made.

Example 2. With a $1+1(1: 2)$ dilution, replicate 1 is found to contain 200 spermatozoa in 400 fields, while replicate 2 contains 230 spermatozoa in 400 fields. The sum of the values $(200+230)$ is 430 in 800 fields and the difference $(230-200)$ is 30 . From Table 2.5 this is seen to be less than that found by chance alone (41), so the values are accepted.

The concentration of spermatozoa in the sample, for a $1+1(1: 2)$ dilution is $\mathrm{C}=$ $(\mathrm{N} / \mathrm{n}) \times(2 / \mathrm{v})$ spermatozoa per $\mathrm{nl}$. If $\mathrm{v}=20 \mathrm{nl}(\times 400$ magnification, see Box 2.13$)$, $\mathrm{C}=(430 / 800) \times(2 / 20)=0.0538$ spermatozoa/nl or 54000 spermatozoa per $\mathrm{ml}$ of semen (to two significant figures). 
Example 3 . With a $1+1(1: 2)$ dilution, replicate 1 is found to contain 50 spermatozoa in the whole chamber, while replicate 2 contains 70 spermatozoa in the whole chamber. The sum of the values $(50+70)$ is 120 in the two chambers and the difference $(70-50)$ is 20 . From Table 2.5 this is seen to be less than that found by chance alone (21), so the values are accepted.

When the entire area of both chambers has been assessed (a total of $50 \mu \mathrm{l}$ ), the concentration of the sample, for a $1+1(1: 2)$ dilution, is $C=(\mathrm{N} / 50) \times 2$ spermatozoa per $\mu l=(120 / 50) \times 2=4.8$ spermatozoa/ $\mu$ l or 4800 spermatozoa per $\mathrm{ml}$ of semen (to two significant figures). As fewer than 400 spermatozoa were counted, report the sampling error for 120 spermatozoa given in Table 2.2 (approximately 10\%).

Example 4. With a $1+1(1: 2)$ dilution, replicate 1 is found to contain 20 spermatozoa in the whole chamber, while replicate 2 contains 18 spermatozoa in the whole chamber. As fewer than 25 spermatozoa were counted, the concentration will be $<2000$ spermatozoa/ml. Report that "38 spermatozoa were seen in the replicates, too few for accurate determination of concentration $(<2000 / \mathrm{ml})$ ".

Example 5. With a $1+1(1: 2)$ dilution, no spermatozoa are found in either replicate. As fewer than 25 spermatozoa were counted, the concentration will be $<2000$ spermatozoa/ml. Report that "No spermatozoa were seen in the replicates, too few for accurate determination of concentration $(<2000 / \mathrm{ml})$ ".

\subsubsection{Calculation of the total numbers of spermatozoa in the ejaculate}

It is recommended to calculate and report the total sperm number per ejaculate, as this parameter provides a measure of the capability of the testes to produce spermatozoa and the patency of the male tract. This is obtained by multiplying the sperm concentration by the volume of the whole ejaculate.

\subsection{Counting of cells other than spermatozoa}

The presence of non-sperm cells in semen may be indicative of testicular damage (immature germ cells), pathology of the efferent ducts (ciliary tufts) or inflammation of the accessory glands (leukocytes). The number of non-sperm cells in semen (epithelial cells, "round cells" (germ cells and leukocytes) or isolated sperm heads and tails) can be estimated in fixed wet preparations by the use of a haemocytometer in the same way as for spermatozoa (see Section 2.8.3). However, semen that has been diluted adequately for counting spermatozoa will normally be too dilute for accurate estimation of non-sperm cells, unless high concentrations are present. The prevalence of round cells relative to spermatozoa can be asssed from slides (see Section 2.12.1). Alternatively, their concentration can be assessed during the estimation of peroxidase-positive cells (see Section 2.18.1.5).

2.12.1 Calculation of the concentration of round cells in semen

The concentration of round cells is calculated relative to that of spermatozoa by assessing fixed and stained semen smears made from undiluted semen (see Section 2.13.2). If $\mathrm{N}$ is the number of round cells counted in the same number of fields as 400 spermatozoa, and $\mathrm{S}$ is the concentration of spermatozoa $\left(10^{6} \mathrm{per} \mathrm{ml}\right)$, then 
the concentration $(\mathrm{C})$ of round cells $\left(10^{6} \mathrm{per} \mathrm{ml}\right)$ can be calculated from the formula $\mathrm{C}=\mathrm{S} \times(\mathrm{N} / 400)$.

2.12.2 Sensitivity of the method

If there are fewer round cells than spermatozoa in the sample (i.e. $<400$ ), the sampling error will exceed $5 \%$. In this case, report the sampling error for the number of cells counted (see Table 2.2). If fewer than 25 round cells are counted, report the number of round cells observed with the comment "Too few for accurate determination of concentration".

\subsubsection{Worked examples}

Example 1. In replicate 1 there are 21 round cells per 200 spermatozoa, while in replicate 2 there are 39 round cells per 200 spermatozoa. The sum of the values $(21+39)$ is 60 and the difference $(39-21)$ is 18 . From Table 2.5 this is seen to exceed the difference expected by chance alone (15), so the results are discarded and new assessments are made.

Example 2. In replicate 1 there are 24 round cells per 200 spermatozoa, while in replicate 2 there are 36 round cells per 200 spermatozoa. The sum of the values $(24+36)$ is 60 and the difference $(36-24)$ is 12 . From Table 2.5 this is seen to be less than that found by chance alone (15), so the values are accepted.

For 60 round cells per 400 spermatozoa and a sperm concentration of $70 \times 10^{6}$ cells per $\mathrm{ml}$, the round cell concentration is $\mathrm{C}=\mathrm{S} \times(\mathrm{N} / 400)$ cells per $\mathrm{ml}=$ $70 \times 10^{6} \times(60 / 400)=10.5 \times 10^{6}$ cells per ml, or $10 \times 10^{6}$ cells per ml (to two significant figures). As fewer than 400 cells were counted, report the sampling error for 60 cells, as given in Table 2.2 (approximately 13\%).

Comment 1: If the estimate of round cell concentration exceeds $1 \times 10^{6}$ per $\mathrm{ml}$, their nature should be assessed by peroxidase activity (see Section 2.18) or leukocyte markers (see Section 3.2) and their concentration measured accurately. It may be possible to identify immature germ cells in well-stained preparations (see Section 2.19).

Comment 2: The total number of round cells in the ejaculate may reflect the severity of the inflammatory or spermatogenic condition. This is obtained by multiplying the concentration of round cells by the volume of the whole ejaculate.

\subsection{Sperm morphology}

Determination of sperm morphology comprises the following steps (which are described in detail in subsequent sections).

- Preparing a smear of semen on a slide (see Section 2.13.2).

- Air-drying, fixing and staining the slide (see Section 2.14). 
- Mounting the slide with a coverslip if the slide is to be kept for a long time (see Sections 2.14.2.4 and 2.14.2.5).

- Examining the slide with brightfield optics at $\times 1000$ magnification with oil immersion (see Sections 2.15 and 2.16).

- Assessing approximately 200 spermatozoa per replicate for the percentage of normal forms (see Section 2.15.1) or of normal and abnormal forms (see Section 2.15.2).

- Comparing replicate values to see if they are acceptably close: if so, proceeding with calculations; if not, re-reading the slides.

\subsubsection{The concept of normal spermatozoa}

The variable morphology of human spermatozoa makes assessment difficult, but observations on spermatozoa recovered from the female reproductive tract, especially in postcoital endocervical mucus (Fredricsson \& Björk, 1977; Menkveld et al., 1990) and also from the surface of the zona pellucida (Menkveld et al., 1991; Liu \& Baker, 1992a) (see Fig. 2.10), have helped to define the appearance of potentially fertilizing (morphologically normal) spermatozoa. By the strict application of certain criteria of sperm morphology, relationships between the percentage of normal forms and various fertility endpoints (time-to-pregnancy (TTP), pregnancy rates in vivo and in vitro) have been established (Eggert-Kruse et al., 1996; J ouannet et al., 1988; Toner et al., 1995; Coetzee et al., 1998; Menkveld et al., 2001; Van Waart et al., 2001; Garrett et al., 2003; Liu et al., 2003), which may be useful for the prognosis of fertility.

The underlying philosophy of the classification system described here is to limit what is identified as normal to the potentially fertilizing subpopulation of spermatozoa prevalent in endocervical mucus. Using these guidelines, the range of percentage normal values for both fertile and infertile men is likely to be $0-30 \%$, with few samples exceeding $25 \%$ normal spermatozoa (Menkveld et al., 2001). This low value will inevitably produce low thresholds; indeed reference limits and thresholds of $3-5 \%$ normal forms have been found in studies of in-vitro fertilization (Coetzee et al., 1998), intrauterine insemination (Van Waart et al., 2001) and in-vivo fertility (Van der Merwe et al., 2005).

The human zona pellucida also selects a subpopulation of morphologically similar spermatozoa, but such "zona-preferred" spermatozoa display a wider range of forms (Liu et al., 1990; Garrett et al., 1997). The percentage of motile spermatozoa in semen from fathers displaying "zona-preferred" morphology is also low (8-25\%) (Liu et al., 2003). 
Fig. 2.10 Morphologically "normal" spermatozoa

$(a, b)$ Shorr-stained spermatozoa recovered from the zona pellucida in vitro. (c) Papanicolaoustained spermatozoa recovered from endocervical mucus after intercourse. Very few defects on the sperm head, midpiece or principal piece are observed. Tails may be curved but not sharply angulated.

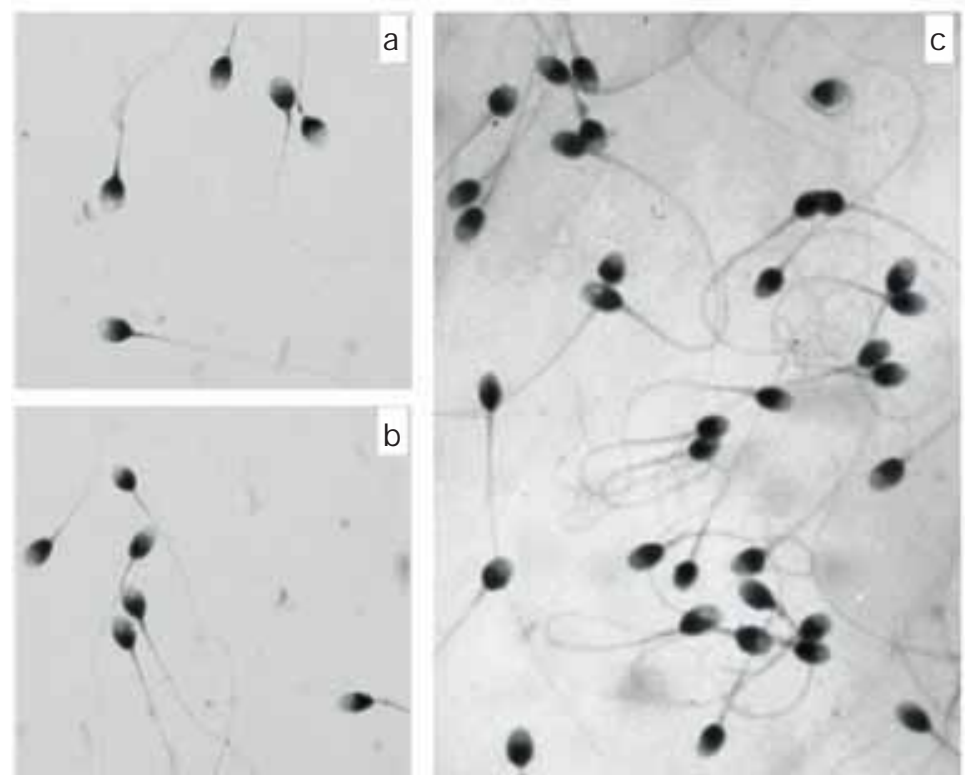

(a, b) Reproduced from Liu et al. (2003) by permission of the European Society of Human Reproduction and Embryology. (c) Reproduced from Menkveld \& Kruger (1990) by permission.

\subsubsection{Preparation of semen smears}

Rapid addition of fixative to semen does not permit adequate visualization of spermatozoa, as they are obscured by denatured seminal proteins. For morphological analysis, it is customary to prepare semen smears that are air-dried before fixation and staining. However, such a process leads to morphological artefacts, since airdrying of semen smears is associated with:

- changes in sperm dimensions: dried, fixed and stained spermatozoa are smaller than live spermatozoa visualized in semen (Katz et al., 1986);

- expansion of immature sperm heads (Soler et al., 2000); and

- loss of osmotically sensitive cytoplasmic droplets (Abraham-Peskir et al., 2002; Cooper et al., 2004), although large amounts of excess residual cytoplasm are retained.

Two or more smears should be made from the fresh semen sample in case there are problems with staining or one slide is broken. Assessment is performed in replicate, preferably on each of the two slides, because there may be significant between-slide variation in sperm morphology.

- Mix the semen sample well (see Box 2.3). 
- Remove an aliquot immediately, allowing no time for the spermatozoa to settle out of suspension.

- Remix the semen sample before removing replicate aliquots.

- Different smearing methods may be used in different conditions (Fig. 2.11).

Fig. 2.11 Semen smearing methods for sperm morphology

(a) "Feathering" method for undiluted semen. The semen drop (S) spreads along the back edge of the angled slide and is pulled forwards over the slide to form the smear. (b) Pipette method for washed samples. A drop of the sperm suspension (SS) is spread over the surface of the slide by pushing the horizontal pipette (P).

(a)

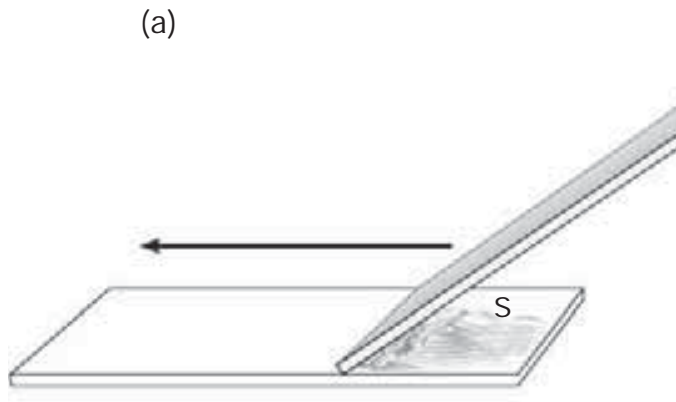

(b)

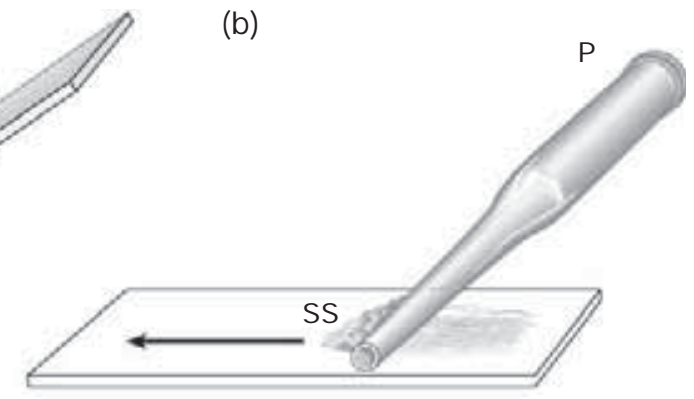

2.13.2.1 Normal semen samples

In this procedure an aliquot of semen is smeared over the whole surface of the slide by the feathering technique (see Figs. 2.11a, 2.12).

1. Clean both surfaces of the frosted slides by rubbing vigorously with lint-free tissue paper.

2. Label the frosted portion with identifying information (e.g. identification number, date) using a pencil with medium-hard lead (HB or No. 2).

3. Apply a 5-10- $\mu$ l aliquot of semen, depending on sperm concentration, to the end of the slide. Use a second slide to pull the drop of semen along the surface of the slide (Figs. 2.11a, 2.12). If the dragging slide is non-frosted, the edges of both ends of the slide can be used to make four different smears.

4. Allow the slides to dry in air and stain them as described in Section 2.14.

Note 1: Pencil lead is stable in fixatives and stains, whereas ink and some permanent markers are not.

Note 2: Do not let the droplet of semen remain on the end of the slide for more than a couple of seconds before smearing. 
Note 3: Be sure to use the slide ahead of the droplet to "pull" the semen across the slide; do not use the slide to "push" the semen from behind.

The quality of the smear (minimal overlap of spermatozoa on the slide) depends on:

- the volume of semen and the sperm concentration: the fewer the spermatozoa, the less likely they are to overlap one another;

- the angle of the dragging slide (Hotchkiss 1945): the smaller the angle, the thinner the smear;

- the speed of smearing (Eliasson 1971): the more rapid the movement, the thicker the smear.

Start with a volume of $10 \mu \mathrm{l}$, an angle of $45^{\circ}$ and a smear of about 1 second. These parameters can then be varied, if necessary, to reduce overlap of spermatozoa on the slide (Menkveld et al., 1990). Feathering works well when semen viscosity is low, but is often unsuitable for extremely viscous semen (see Fig. 2.12 and Section 2.13.2.3).

Fig. 2.12 Preparing a normal semen smear

To get the feel for the motion, place the dragging slide at an angle of $45^{\circ}$ and move it into contact with the aliquot of semen (left panel), which runs along the edge of the slide (middle panel). B ring the dragging slide slowly back (over approximately 1 second) along the length of the slide to produce the smear (right panel).
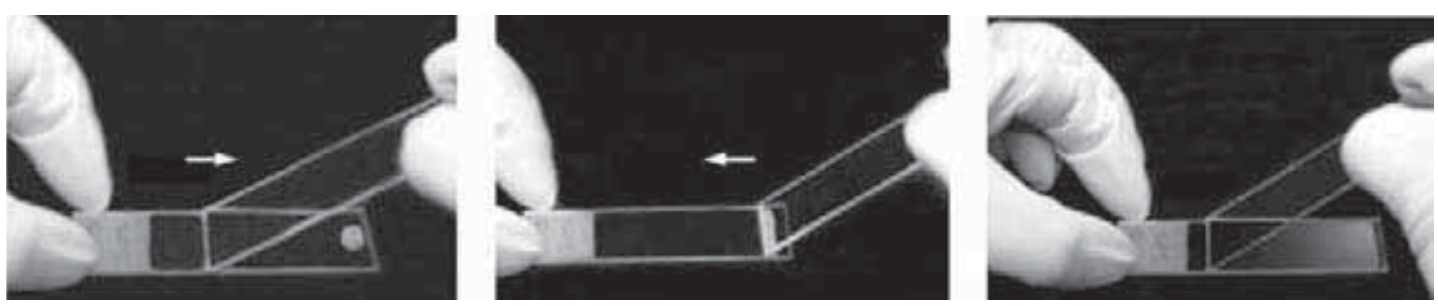

Photographs courtesy of C Brazil.

With low sperm concentrations $\left(<2 \times 10^{6} / \mathrm{ml}\right)$, viscous or debris-laden samples, or when computer-assisted morphology is to be done (see Section 3.5.4), different approaches may be needed.

\subsubsection{Samples with low sperm concentration}

If the concentration of spermatozoa is low (e.g. $<2 \times 10^{6} / \mathrm{ml}$ ), concentrate the sample:

1. Centrifuge the sample at $600 \mathrm{~g}$ for 10 minutes.

2. Remove most of the supernatant. 
3. Resuspend the pellet in the remainder of the supernatant by gentle pipetting.

4. Obtain the highest sperm concentration possible, not exceeding approximately $50 \times 10^{6} / \mathrm{ml}$.

5. Treat as a normal sample (see Section 2.13.2.1).

Note: Centrifugation may affect sperm morphology and its use must be recorded.

\subsubsection{Viscous semen samples}

Sometimes it is difficult to prepare good smears because the seminal plasma is highly viscous, resulting in smears of uneven thickness. Viscous samples can be treated in the same way as poorly liquefied samples (see Section 2.3.1.1) or by washing (see Section 2.13.2.4).

Note: These procedures may affect sperm morphology and their use must be recorded.

2.13.2.4 Washing debris-laden or viscous semen samples and to reduce background for computer-aided sperm morphometric assessment

Debris and a large amount of particulate material (such as in viscous samples) may cause spermatozoa to lie with their heads on edge, making them difficult to categorize. These samples may be washed, as follows.

1. Dilute an aliquot of semen $(0.2-0.5 \mathrm{ml}$, depending on sperm concentration) to $10 \mathrm{ml}$ with normal saline $(0.9 \mathrm{~g}$ of sodium chloride $(\mathrm{NaCl})$ per $100 \mathrm{ml}$ of purified water) at room temperature.

2. Centrifuge at $800 \mathrm{~g}$ for 10 minutes.

3. Decant most of the supernatant.

4. Resuspend the pellet in the remaining supernatant (typically $20-40 \mu \mathrm{l}$ ) by gentle pipetting.

5. Make a smear of the suspension by spreading $5-10 \mu l$ of sperm suspension on a microscope slide with a Pasteur pipette (see Fig. 2.11b).

6. Scan the slide with phase-contrast optics at $\times 400$ magnification to ensure that the smear is evenly spread.

7. Check that there are at least 40 spermatozoa per $\times 400$ field with no clumping or overlapping.

8. Allow the slides to dry in air and stain as described in Section 2.14. 
Note 1: If too many spermatozoa are overlapping on the slide, make another smear using a smaller aliquot of semen.

Note 2: If the spermatozoa are too sparse on the slide, make another smear using a larger aliquot of semen.

Note 3: Washing the sample may affect sperm morphology and the procedure must be recorded.

Comment: Leaving the semen to liquefy for longer than 30 minutes before making the smears may reduce background staining.

\subsection{Staining methods}

Once the semen smears have been air-dried, they should be fixed and stained to highlight details of the spermatozoa. The use of the Papanicolaou, Shorr or DiffQuik stain is recommended.

With all three stains in brightfield optics, the head is stained pale blue in the acrosomal region and dark blue in the post-acrosomal region. The midpiece may show some red staining and the tail is stained blue or reddish. Excess residual cytoplasm, usually located behind the head and around the midpiece, is stained pink or red (Papanicolaou stain) or reddish-orange (Shorr stain).

Comment: Rapid staining methods, in which a drop of semen is added to fixative and stain already on the slide, are commercially available. These are not recommended, however, because without the even distribution of spermatozoa provided by the smearing technique, it is not possible to observe the details necessary for the morphological classification described here.

\subsubsection{Traditional fixation and sequential staining}

This involves the following steps:

- ethanol

- graded ethanol

- purified water

- haematoxylin

- tap water

- acidic ethanol

- tap water to fix the cells; it also dehydrates them;

to rehydrate the fixed smears gradually to permit water-soluble haematoxylin staining;

to rehydrate dried smears to permit water-soluble haematoxylin staining;

to stain the nucleus blue;

to remove unbound nuclear haematoxylin;

to remove non-specifically bound dye from the cytoplasm (destaining);

to reduce acidity and return blue colour to the nucleus; 
- Scott's solution

- ethanol

- Orange $G$

- $\mathrm{EA}-50$

- graded ethanol

- xylene to return blue colour to the nucleus (if tap water is insufficient);

to dehydrate smears to permit ethanol-soluble Orange G / EA-50 staining;

to stain the cytoplasm pink;

to stain the cytoplasm pink;

to dehydrate the stained smears gradually to permit the use of ethanol-soluble mountants;

to permit the use of ethanol-insoluble mountants (see Box 2.14).

\section{Box 2.14 Mounting media}

Slides can be viewed unmounted or mounted (without or with a coverslip attached). Mounting the slides permits long-term storage, so that they can be reassessed if necessary and used in an internal quality control programme. The refractive index (RI) of mountants after drying (1.50-1.55) is similar to that of glass (1.50-1.58), and the best optical quality comes with the use of immersion oil with a similar RI (1.52).

\subsubsection{Papanicolaou staining procedure for sperm morphology}

The Papanicolaou stain gives good staining of spermatozoa and other cells. It stains the acrosomal and post-acrosomal regions of the head, excess residual cytoplasm, the midpiece and the principal piece. The modified staining technique described here has proved useful in the analysis of sperm morphology and in the examination of immature germ cells and non-sperm cells (see Plates 1-14). Routine procedures have been modified to work without ether (as fixative) or xylene (for mounting) (ESHRE/NAFA, 2002) (see Section 2.14.2.4). Slides stained using the Papanicolaou procedure can be permanently mounted and stored for future use in internal quality control programmes. If stored in the dark, they should be stable for months or years.

The following method was used to prepare the plates in this manual, from slides that were mounted in an ethanol-insoluble mountant.

\subsubsection{Reagents}

1. Papanicolaou constituent stains: commercially available or see Appendix 4, section A4.10.

2. Acidic ethanol: add $1.0 \mathrm{ml}$ of concentrated hydrochloric acid to $200 \mathrm{ml}$ of $70 \%(v / v)$ ethanol.

3. Xylene:ethanol, $1+1$ (1:2): mix equal parts of $100 \%$ ethanol and xylene.

Note 1: Xylene is a health hazard and should be used in a fume cupboard.

Note 2: Smears should be air-dried for at least 4 hours, but can be stored for up to 1 week, before fixing and staining. 


\subsubsection{Fixing the air-dried semen smear}

1. Immerse slides in $95 \%(\mathrm{v} / \mathrm{v})$ ethanol for at least 15 minutes.

\subsubsection{Staining the fixed semen smear}

Sequentially immerse the slides in:

1. Ethanol $80 \%(\mathrm{v} / \mathrm{v})$

2. Ethanol $50 \%(\mathrm{v} / \mathrm{v})$

3. Purified water

4. Harris's haematoxylin

5. Purified water

6. Acidic ethanol

7. Running cold tap water

8. Ethanol $50 \%(\mathrm{v} / \mathrm{v})$

9. Ethanol $80 \%(\mathrm{v} / \mathrm{v})$

10. Ethanol $95 \%(\mathrm{v} / \mathrm{v})$

11. G-6 orange stain

12. Ethanol $95 \%(\mathrm{v} / \mathrm{v})$

13. Ethanol $95 \%(v / v)$

14. Ethanol $95 \%(v / v)$

15. EA-50 green stain

16. Ethanol 95\% (v/v)

17. Ethanol $95 \%(v / v)$

18. Ethanol $100 \%$

19. Ethanol $100 \%$
30 seconds

30 seconds

30 seconds

4 minutes

30 seconds

4-8 dips*

5 minutes

30 seconds

30 seconds

At least 15 minutes

1 minute

30 seconds

30 seconds

30 seconds

1 minute

30 seconds

30 seconds

15 seconds

15 seconds

*One dip corresponds to an immersion of about 1 second.

Note 1: Ethanol fixation causes dehydration of the cells. Therefore smears taken directly from the fixation step in $95 \%$ ethanol to staining may need only 10 seconds in the $80 \%$ ethanol, whereas smears that have air-dried after fixation must remain longer (2-3 minutes) in the $50 \%$ ethanol.

Note 2: In Step 6 above, start with 4 dips and continue until results are satisfactory. This is a critical step, as the duration of destaining dramatically alters the final stain intensity. If this step is omitted, spermatozoa and background will be dark. Increasing the number of dips will make spermatozoa and background fainter.

Note 3: The slides can be viewed unmounted or mounted. 
2.14.2.4 Treating the stained semen smear before mounting

There are two kinds of fluid for mounting the preparation: ethanol-soluble and ethanol-insoluble mountants.

- Use ethanol-soluble mounting media directly on smears still moist with ethanol.

- For ethanol-insoluble mounting media, take slides directly from step 19 above through the following steps (to be performed in a fume cupboard):

1. Xylene:ethanol, $1+1(1: 2) \quad 1$ minute

2. Xylene $100 \%$

Remove one slide at a time from the xylene staining container and allow it to drain for only 1-2 seconds, as the slide should be quite wet with xylene when mounting.

2.14.2.5 Mounting the stained semen smears

1. Add two or three small drops of mounting medium to the slide.

2. Place a coverslip ( $24 \mathrm{~mm} \times 50 \mathrm{~mm}$ or $24 \mathrm{~mm} \times 60 \mathrm{~mm}$ are most convenient) directly on the smear.

3. Position the coverslip so that contact with the mounting medium begins from one long side, in order to prevent air bubbles being trapped.

4. If necessary, press gently on the top of the coverslip to help move bubbles to the edge of the slide.

5. Wipe off excess xylene (if used) from underneath the slide.

6. Allow the mounted smear to dry horizontally in a slide drying rack or on absorbant paper for 24 hours in a fume cupboard.

2.14.3 Shorr staining procedure for sperm morphology

The Shorr stain provides similar percentages of normal forms as the Papanicolaou stain (Meschede et al., 1993).

\subsubsection{Reagents}

1. Harris haematoxylin: Papanicolaou No. 1.

2. Shorr solution: buy readymade or prepare as follows. Dissolve $4 \mathrm{~g}$ of Shorr powder in $220 \mathrm{ml}$ of warm $50 \%$ ( $/ \mathrm{v} / \mathrm{v}$ ) ethanol. Allow to cool, add $2.0 \mathrm{ml}$ of glacial acetic acid (in fume cupboard) and filter.

3. Acetic ethanol: add $25 \mathrm{ml}$ of glacial acetic acid to $75 \mathrm{ml}$ of $95 \%(\mathrm{v} / \mathrm{v})$ ethanol.

4. Ammoniacal ethanol: add $5 \mathrm{ml}$ of $25 \%(\mathrm{v} / \mathrm{v})$ ammonium hydroxide to $95 \mathrm{ml}$ of $75 \%(v / v)$ ethanol.

2.14.3.2 Fixing the air-dried semen smear

Immerse slides in acetic ethanol or $75 \%(v / v)$ ethanol for 1 hour. 
2.14.3.3 Staining the fixed semen smear

Sequentially immerse the slides in:
1. Running tap water
12-15 dips*
2. Haematoxylin
1-2 minutes
3. Running tap water
12-15 dips*
4. Ammoniacal ethanol
10 dips*
5. Running tap water
12-15 dips*
6. Ethanol $50 \%(\mathrm{v} / \mathrm{v})$
5 minutes
7. Shorr stain
3-5 minutes
8. Ethanol $50 \%(\mathrm{v} / \mathrm{v})$
5 minutes
9. Ethanol $75 \%(\mathrm{v} / \mathrm{v})$
5 minutes
10. Ethanol 95\% (v/v)
5 minutes

* One dip corresponds to an immersion of about 1 second.

Note: The slides can be viewed unmounted or mounted.

\subsubsection{Mounting the stained semen smear}

See Sections 2.14.2.4 and 2.14.2.5.

\subsubsection{Rapid staining procedure for sperm morphology}

Rapid staining methods are particularly useful for clinical laboratories that need to provide results on the day of analysis. Several differential staining sets are available (Kruger et al., 1987). Some smears stained by rapid procedures have high background staining and may be of lower quality than those stained with Papanicolaou stain.

\subsubsection{Reagents}

1. Diff-Quik rapid staining kit consisting of:

a) fixative reagent (triarylmethane dye dissolved in methanol);

b) staining solution 1 (eosinophilic xanthene);

c) staining solution 2 (basophilic thiazine).

2. Fixative: $1.8 \mathrm{mg}$ of triarylmethane dissolved in $1000 \mathrm{ml}$ of $95 \%(\mathrm{v} / \mathrm{v})$ methanol, optional.

3. Fixative: methanol $95 \%(\mathrm{v} / \mathrm{v})$, optional. 


\subsubsection{Fixing the air-dried semen smear}

Immerse slides in triarylmethane fixative (as provided in the Diff-Quik kit or prepared as above) for 15 seconds or $95 \%$ methanol alone for 1 hour. Drain the excess solution by placing slides vertically on absorbent paper.

\subsubsection{Staining the fixed semen smear}

Sequentially immerse the slides in:
1. Rapid stain solution 1
10 seconds
2. Rapid stain solution 2
5 seconds
3. Running tap water
10 to 15 times to remove excess stain

Drain the excess solution at each step by placing slides vertically on absorbent paper.

Note 1: The slides can be viewed unmounted or mounted.

Note 2: If there is high background staining, an aliquot of the semen sample should be washed (see Section 2.13.2.4) and new slides prepared and stained. Washing may affect sperm morphology and its use must be recorded.

\subsubsection{Mounting the stained semen smear}

See Sections 2.14.2.4 and 2.14.2.5.

\subsection{Examining the stained preparation}

With stained preparations, a $\times 100$ oil-immersion brightfield objective and at least a $\times 10$ eyepiece should be used. Clearer images are obtained when a fluid of similar refractive index $(\mathrm{RI})$ to those of cells (approximately 1.5$)$ and glass (1.50-1.58) is placed between the lens and the unmounted section or glass coverslip. This is usually immersion oil (RI 1.52). Mounting media have similar refractive indices (1.50-1.55: see Box 2.14).

2.15.1 Classification of normal sperm morphology

Assessment of sperm morphology is associated with a number of difficulties related to lack of objectivity, variation in interpretation or poor performance in external quality-control assessments (see Section 7.13.2). The method recommended here is a simple normal/abnormal classification, with optional tallying of the location of abnormalities in abnormal spermatozoa. The criteria overpage should be applied when assessing the morphological normality of the spermatozoon (Kruger et al., 1986; Menkveld et al., 1990; Coetzee et al., 1998). The reference limit given (Section 2.17.3) is valid only when the technique described below is used. 
Spermatozoa consist of a head, neck, middle piece (midpiece), principal piece and endpiece. As the endpiece is difficult to see with a light microscope, the cell can be considered to comprise a head (and neck) and tail (midpiece and principal piece). For a spermatozoon to be considered normal, both its head and tail must be normal. All borderline forms should be considered abnormal.

- The head should be smooth, regularly contoured and generally oval in shape. There should be a well-defined acrosomal region comprising $40-70 \%$ of the head area (Menkveld et al., 2001). The acrosomal region should contain no large vacuoles, and not more than two small vacuoles, which should not occupy more than $20 \%$ of the sperm head. The post-acrosomal region should not contain any vacuoles.

- The midpiece should be slender, regular and about the same length as the sperm head. The major axis of the midpiece should be aligned with the major axis of the sperm head. Residual cytoplasm is considered an anomaly only when in excess, i.e. when it exceeds one third of the sperm head size (Mortimer \& Menkveld, 2001).

- The principal piece should have a uniform calibre along its length, be thinner than the midpiece, and be approximately $45 \mu \mathrm{m}$ long (about 10 times the head length). It may be looped back on itself (see Fig. $2.10 \mathrm{c}$ ), provided there is no sharp angle indicative of a flagellar break.

Comment 1: With this technique, the form of the sperm head is more important than its dimensions, unless these are grossly abnormal.

Comment 2: An eyepiece micrometer may be useful for distinguishing between normally and abnormally sized sperm heads.

Comment 3: The head dimensions of 77 Papanicolaou-stained spermatozoa (stained by the procedure given in Section 2.14.2 and classified as normal by the criteria given here), measured by a computerized system (coefficient of variation for repeated measurements $2-7 \%$ ) had the following dimensions: median length $4.1 \mu \mathrm{m}, 95 \% \mathrm{Cl} 3.7-4.7$; median width $2.8 \mu \mathrm{m}, 95 \% \mathrm{Cl} 2.5-3.2$; median length-towidth ratio $1.5,95 \% \mathrm{Cl} 1.3-1.8$.

Comment 4: The midpieces of 74 Papanicolaou-stained spermatozoa (stained by the procedure given in Section 2.14.2 and classified as normal by the criteria given here) and measured by the same computerized system had the following dimensions: median length $4.0 \mu \mathrm{m}, 95 \% \mathrm{Cl} 3.3-5.2$; median width $0.6 \mu \mathrm{m}, 95 \% \mathrm{Cl}$ 0.5-0.7.

Comment 5: Coiled tails ( $>360^{\circ}$; see Fig. $2.13 \mathrm{~m}$ ) may indicate epididymal dysfunction (Pelfrey et al., 1982).

This assessment of normal sperm morphology can best be applied by learning to recognize the subtle variations in shape of the entire spermatozoon (normal/borderline sperm heads and tails; see Section 2.16, Plates 1-12 and their commentaries). 


\subsubsection{Classification of abnormal sperm morphology}

Human semen samples contain spermatozoa with different kinds of malformations. Defective spermatogenesis and some epididymal pathologies are commonly associated with an increased percentage of spermatozoa with abnormal shapes. The morphological defects are usually mixed. Abnormal spermatozoa generally have a lower fertilizing potential, depending on the types of anomalies, and may also have abnormal DNA. Morphological defects have been associated with increased DNA fragmentation (Gandini et al., 2000), an increased incidence of structural chromosomal aberrations (Lee et al., 1996), immature chromatin (Dadoune et al., 1988) and aneuploidy (Devillard et al., 2002; Martin et al., 2003). Emphasis is therefore given to the form of the head, although the sperm tail (midpiece and principal piece) is also considered.

The following categories of defects should be noted (see Fig. 2.13).

- Head defects: large or small, tapered, pyriform, round, amorphous, vacuolated (more than two vacuoles or $>20 \%$ of the head area occupied by unstained vacuolar areas), vacuoles in the post-acrosomal region, small or large acrosomal areas ( $<40 \%$ or $>70 \%$ of the head area), double heads, or any combination of these.

- Neck and midpiece defects: asymmetrical insertion of the midpiece into the head, thick or irregular, sharply bent, abnormally thin, or any combination of these.

- Principal piece defects: short, multiple, broken, smooth hairpin bends, sharply angulated bends, of irregular width, coiled, or any combination of these.

- Excess residual cytoplasm (ERC): this is associated with abnormal spermatozoa produced from a defective spermatogenic process. Spermatozoa characterized by large amounts of irregular stained cytoplasm, one third or more of the sperm head size, often associated with defective midpieces (Mortimer \& Menkveld, 2001) are abnormal. This abnormal excess cytoplasm should not be called a cytoplasmic droplet (Cooper, 2005).

Comment 1: Cytoplasmic droplets (membrane-bound vesicles on the midpiece at the head-neck junction) are normal components of physiologically functional human spermatozoa. If swollen, they may extend along the length of the midpiece, as observed by phase-contrast, differential-interference-contrast and X-ray microscopy of living cells in semen, cervical mucus and medium (Abraham-Peskir et al., 2002; Fetic et al., 2006).

Comment 2: Cytoplasmic droplets are osmotically sensitive and are not well preserved by routine air-drying procedures (Chantler $\&$ Abraham-Peskir, 2004; Cooper et al., 2004). They are not obvious in stained preparations, where they may appear as small distensions of the midpiece. Cytoplasmic droplets are less than one third the size of the sperm head in fixed and stained preparations (Mortimer \& Menkveld, 2001) and are not considered abnormal. 
Fig. 2.13 Schematic drawings of some abnormal forms of human spermatozoa

A. Head defects

(a)

Tapered

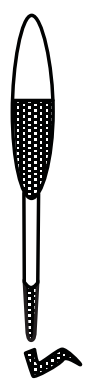

(b) Pyriform

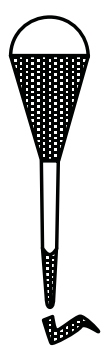

(c)

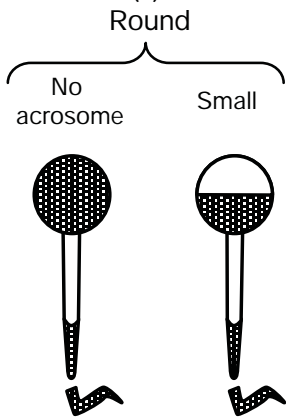

(d)

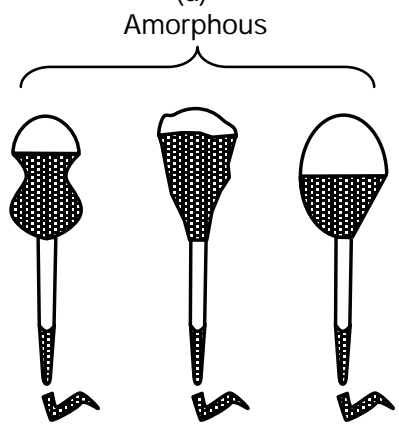

(e) Vacuolated

(f)

Small acrosomal
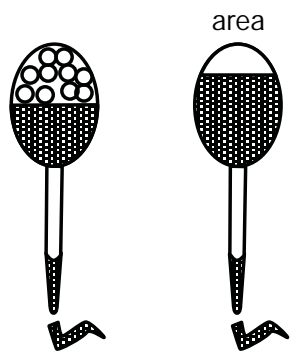

\section{B. Neck and midpiece defects}

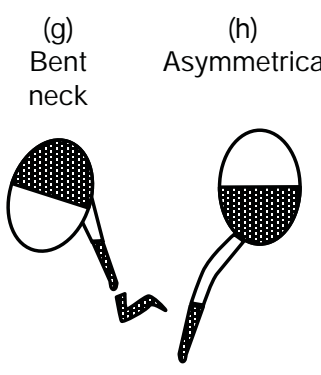

(i) (j)

Thick insertion

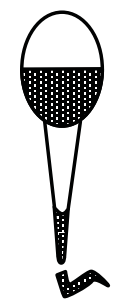

(j)
Thin

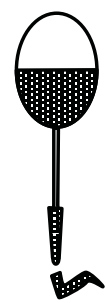

\section{Tail defects}

(k)

Short

(I)

Bent

(m)

Coiled
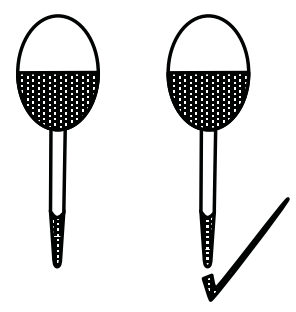

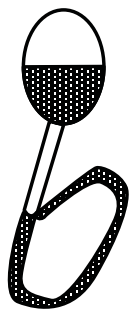

\section{Excess residual} cytoplasm

(n) $>$ one third head

Adapted from Kruger et al., 1993 and reproduced by permission of MQ Medical.

\subsection{Morphology plates 1-14}

All the micrographs in Plates 1-14 were assessed by strict application of the stringent morphological criteria presented above. The analysis of sperm morphology is subjective and particularly difficult to standardize, since it attempts to draw an artificial cut-off point between normal and abnormal cells, on the basis of a multitude of characteristics of sperm heads and tails. The plates that follow were assessed by a single expert, Dr Thinus Kruger. The assessments have been supplemented with additional comments to ensure consistency of notation of all abnormalities.

Opposite each colour plate is a table describing the morphology assessment of each spermatozoon pictured. The table indicates whether the head shape is normal or abnormal, provides details of head abnormalities other than shape, indicates whether the midpiece or principal piece is normal in form, and whether the spermatozoon can be considered normal overall. Other relevant remarks are listed under "comments". The comments are further explained in Table 2.6. 
Table 2.6 Explanations used in commentaries to Plates 1-14

\begin{tabular}{|c|c|}
\hline$<40 \%$ acr & less than $40 \%$ of the sperm head is occupied by the acrosome \\
\hline$>70 \%$ acr & more than $70 \%$ of the sperm head is occupied by the acrosome \\
\hline >one third & abnormal cytoplasm (more than one third of head size) (ERC) \\
\hline$<$ one third & normal cytoplasm (less than one third of head size) (CD) \\
\hline abnormal & self-explanatory \\
\hline amorphous & head shape (see Fig. 2.13d) \\
\hline bacilli & bacteria \\
\hline bent & unnaturally sharp angulation (see Figs $2.13 \mathrm{~g}$ and $\mathrm{j}$ ) \\
\hline coiled & self-explanatory \\
\hline$C D$ & cytoplasmic droplet \\
\hline cytoplasm & $\begin{array}{l}\text { either excess residual cytoplasm or cytoplasmic droplet, } \\
\text { depending on size }\end{array}$ \\
\hline degenerating leukocyte & self-explanatory \\
\hline degenerating spermatid & self-explanatory \\
\hline defect & self-explanatory \\
\hline double & self-explanatory \\
\hline epithelial cell & from male duct system \\
\hline ERC & excess residual cytoplasm (see Fig. $2.13 n$ ) \\
\hline flat & base of sperm head not oval \\
\hline focus & out of focus (not assessed) \\
\hline if $\mathrm{PP} O \mathrm{OK}$ & $\begin{array}{l}\text { not all the principal piece is seen in the micrograph (but if it were } \\
\text { normal, the spermatozoon would be considered normal) }\end{array}$ \\
\hline insert & the site of insertion of the tail is to one side of the long axis of the head \\
\hline irreg & irregular in outline \\
\hline looped & tail bent back on itself \\
\hline macrophage & phagocytic leukocyte \\
\hline monocyte & agranular leukocyte \\
\hline spermatid & immature germ cell \\
\hline no acro & acrosome absent \\
\hline normal & resembling those found in endocervical mucus \\
\hline not assessed & because of overlap or poor focus \\
\hline overlapping & heads obscured by tail \\
\hline PA vac & vacuole in the post-acrosomal region \\
\hline pinhead & not a spermatozoon; no chromatin present \\
\hline polymorph & polymorphonuclear leukocyte \\
\hline pyriform & head shape (see Fig. 2.13b) \\
\hline round & head shape (see Fig. 2.13c) \\
\hline side view & spermatozoon seen edge on \\
\hline small & head size \\
\hline spermatid & immature germ cell \\
\hline spermatocyte & immature germ cell \\
\hline tapered & head shape (see Fig. 2.13a) \\
\hline thick & self-explanatory \\
\hline too long & self-explanatory \\
\hline vac & vacuole \\
\hline$>2$ vac & more than two vacuoles \\
\hline
\end{tabular}


Plate 1
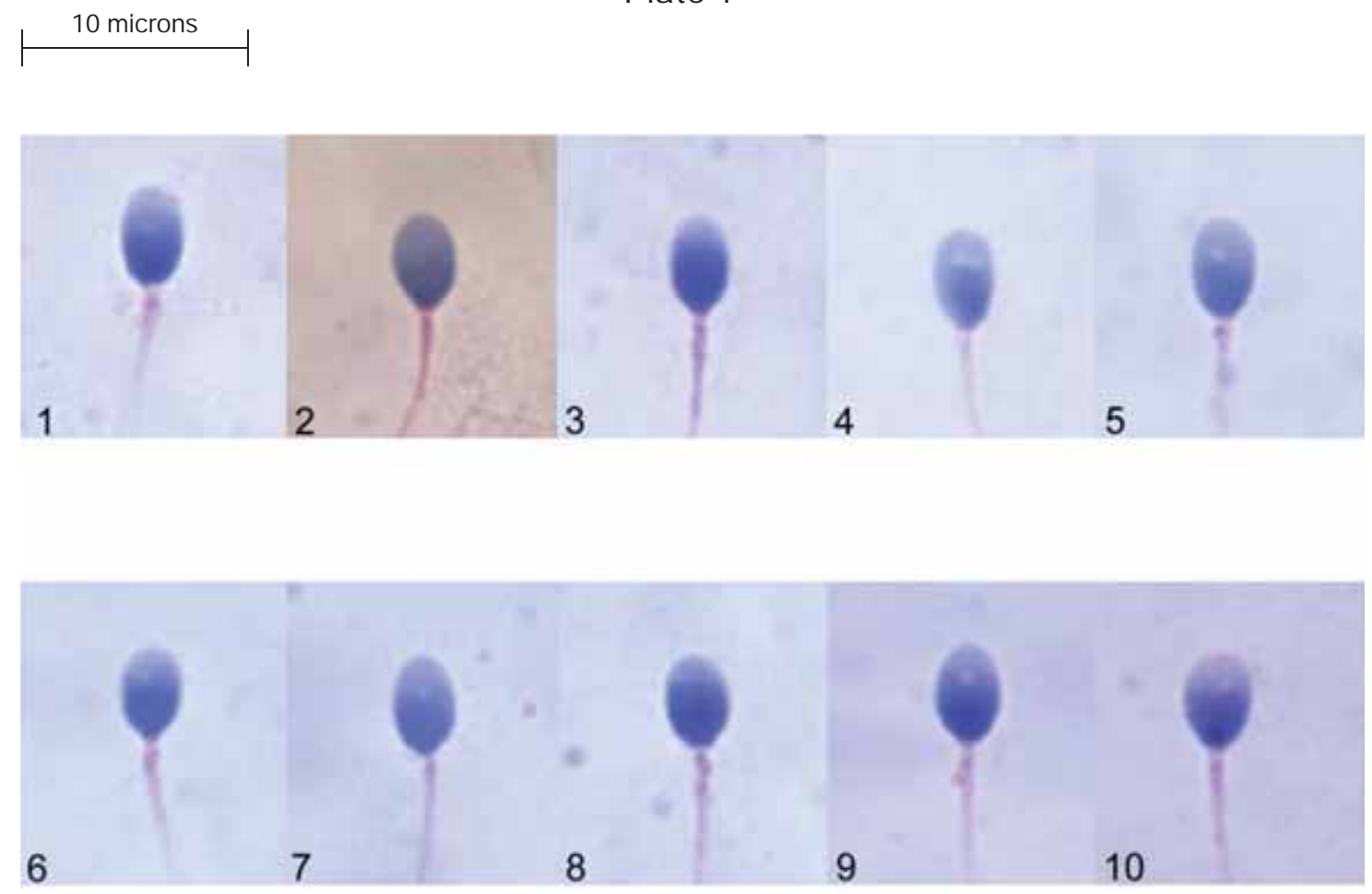
Morphology assessment of spermatozoa in Plate 1

\begin{tabular}{|c|c|c|c|c|c|c|}
\hline Sperm & $\begin{array}{l}\text { Head } \\
\text { shape }\end{array}$ & $\begin{array}{l}\text { Other head } \\
\text { comments }\end{array}$ & $\begin{array}{l}\text { Midpiece } \\
\text { comments }\end{array}$ & $\begin{array}{l}\text { Principal } \\
\text { piece } \\
\text { comments }\end{array}$ & $\begin{array}{l}\text { Overall sperm } \\
\text { classification }\end{array}$ & Comments \\
\hline 1 & normal & & normal & & normal & if $P P O K$ \\
\hline 2 & normal & & normal & & normal & if PP OK \\
\hline 3 & normal & & normal & & normal & if PP OK \\
\hline 4 & normal & & normal & & normal & if $\mathrm{PP} O K$ \\
\hline 5 & normal & & normal & & normal & if $\mathrm{PP} O \mathrm{OK}$ \\
\hline 6 & normal & & normal & & normal & if $\mathrm{PP} O \mathrm{OK}$ \\
\hline 7 & normal & & normal & & normal & if $\mathrm{PP} O K$ \\
\hline 8 & normal & & normal & & normal & if $\mathrm{PP} O K$ \\
\hline 9 & normal & & normal & & normal & if PP OK \\
\hline 10 & normal & & normal & & normal & if $\mathrm{PP} \mathrm{OK}$ \\
\hline 11 & normal & & normal & & normal & if $\mathrm{PP} O \mathrm{OK}$ \\
\hline 12 & normal & & normal & & normal & if $\mathrm{PP} \mathrm{OK}$ \\
\hline 13 & normal & & normal & & normal & if $\mathrm{PP} O K$ \\
\hline 14 & normal & & normal & & normal & if $\mathrm{PP} O K$ \\
\hline 15 & normal & & normal & & normal & if $\mathrm{PP} \mathrm{OK}$ \\
\hline 16 & normal & & normal & & normal & if $\mathrm{PP} \mathrm{OK}$ \\
\hline 17 & normal & & normal & & normal & if $\mathrm{PP} O K$ \\
\hline 18 & normal & & normal & & normal & if $\mathrm{PP} O K$ \\
\hline 19 & normal & & normal & & normal & if $\mathrm{PP} \mathrm{OK}$ \\
\hline 20 & normal & & normal & & normal & if $\mathrm{PP} O K$ \\
\hline
\end{tabular}




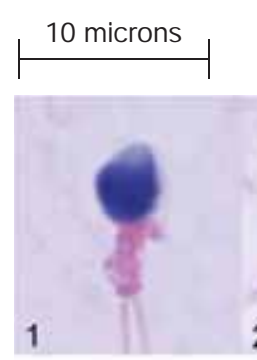

Plate 2
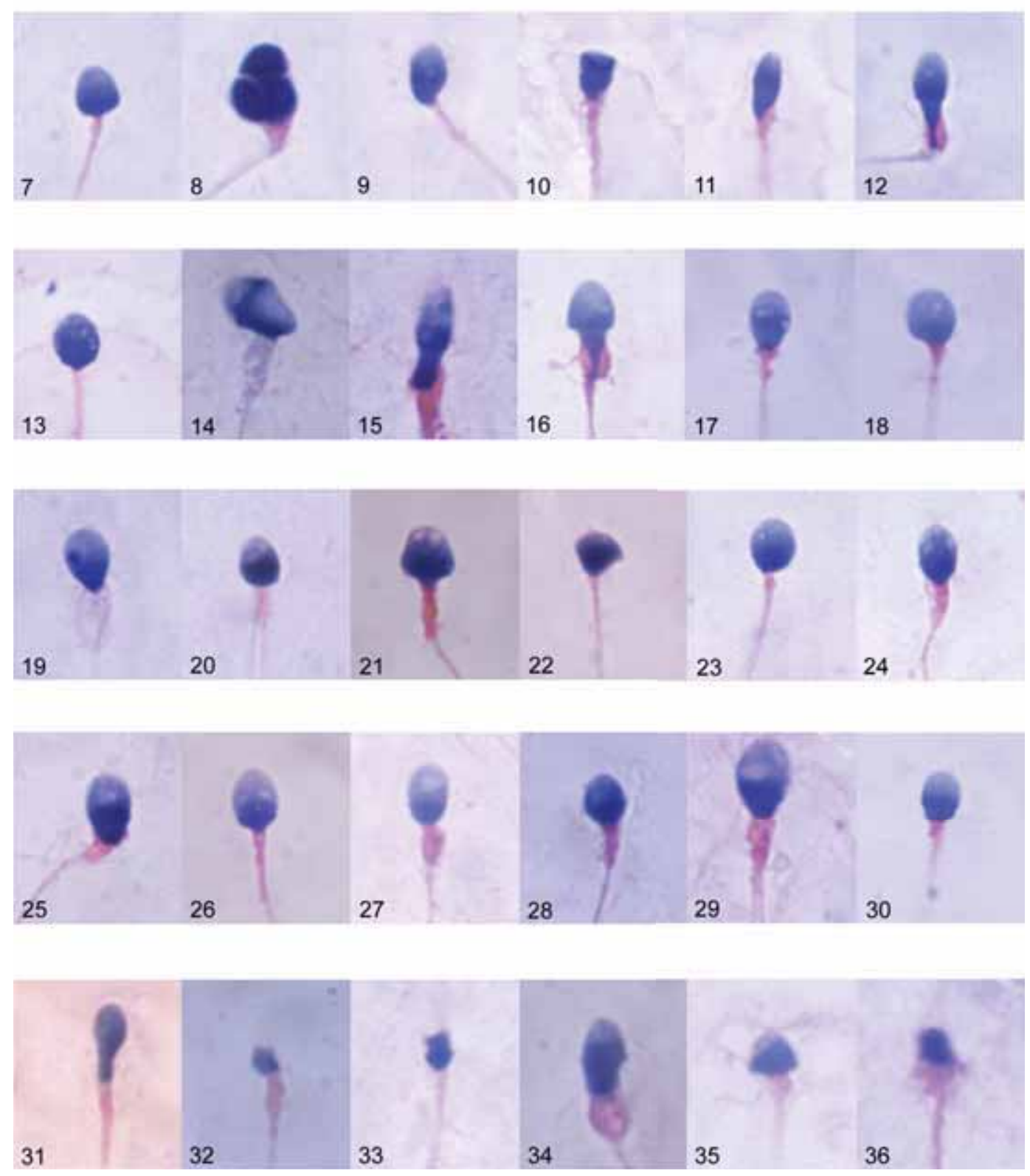

Micrographs courtesy of C Brazil. 
Morphology assessment of spermatozoa in Plate 2

\begin{tabular}{|c|c|c|c|c|c|c|}
\hline Sperm & $\begin{array}{l}\text { Head } \\
\text { shape }\end{array}$ & $\begin{array}{l}\text { Other head } \\
\text { comments }\end{array}$ & $\begin{array}{l}\text { Midpiece } \\
\text { comments }\end{array}$ & $\begin{array}{l}\text { Principal } \\
\text { piece } \\
\text { comments }\end{array}$ & $\begin{array}{l}\text { Overall sperm } \\
\text { classification }\end{array}$ & Comments \\
\hline 1 & abnormal & & thick & double & abnormal & \\
\hline 2 & abnormal & & irreg & & abnormal & side view \\
\hline 3 & abnormal & pyriform & $\begin{array}{c}\text { bent, irreg, } \\
\text { ERC }\end{array}$ & & abnormal & >one third \\
\hline 4 & abnormal & & & & abnormal & \\
\hline 5 & abnormal & pyriform & & & abnormal & \\
\hline 6 & abnormal & & & & abnormal & \\
\hline 7 & abnormal & & & & abnormal & \\
\hline 8 & abnormal & & thick & & abnormal & \\
\hline 9 & abnormal & & insert & & abnormal & \\
\hline 10 & abnormal & & & & abnormal & \\
\hline 11 & abnormal & & & & abnormal & \\
\hline 12 & abnormal & pyriform & & bent & abnormal & \\
\hline 13 & abnormal & $\begin{array}{l}>2 \text { vac, } \\
\text { PA vac }\end{array}$ & & & abnormal & \\
\hline 14 & abnormal & & thick & & abnormal & \\
\hline 15 & abnormal & pyriform & thick, ERC & & abnormal & >one third \\
\hline 16 & abnormal & pyriform & ERC & & abnormal & >one third \\
\hline 17 & normal & PA vac & & & abnormal & \\
\hline 18 & abnormal & & thick, insert & & abnormal & \\
\hline 19 & abnormal & & abnormal & & abnormal & \\
\hline 20 & abnormal & & thick & & abnormal & \\
\hline 21 & abnormal & & thick & & abnormal & \\
\hline 22 & abnormal & & & & abnormal & \\
\hline 23 & abnormal & & & & abnormal & \\
\hline 24 & normal & $>2$ vac & thick & & abnormal & \\
\hline 25 & abnormal & & thick, bent & & abnormal & \\
\hline 26 & abnormal & & thick & & abnormal & \\
\hline 27 & abnormal & $>70 \%$ acr & thick & & abnormal & \\
\hline 28 & abnormal & & thick & & abnormal & \\
\hline 29 & abnormal & & thick & & abnormal & \\
\hline 30 & abnormal & & thick & & abnormal & \\
\hline 31 & abnormal & pyriform & thick & & abnormal & \\
\hline 32 & abnormal & small & thick & & abnormal & \\
\hline 33 & abnormal & small & thick & & abnormal & \\
\hline 34 & abnormal & & ERC & & abnormal & >one third \\
\hline 35 & abnormal & & thick & & abnormal & \\
\hline 36 & abnormal & & thick & & abnormal & \\
\hline
\end{tabular}


10 microns

Plate 3

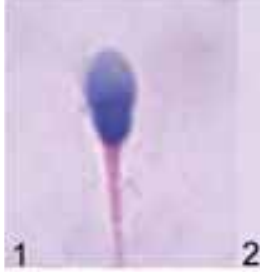

3
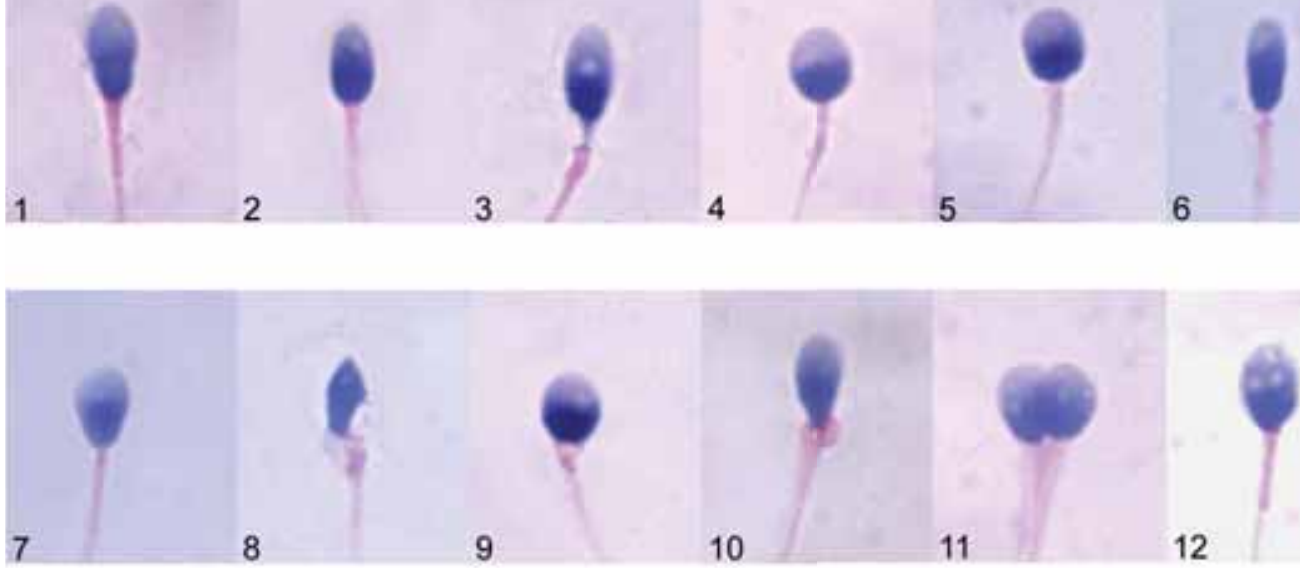

9

10

11

12

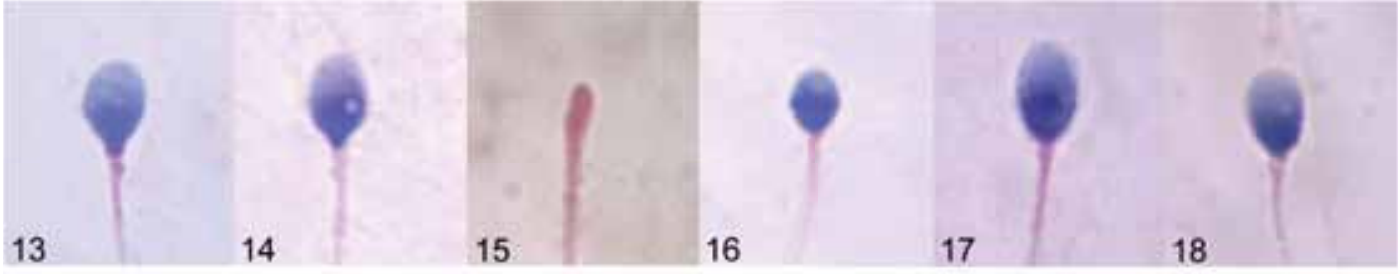

19

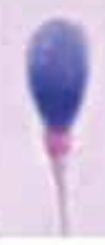

20

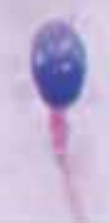

21

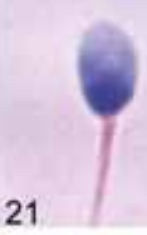

22
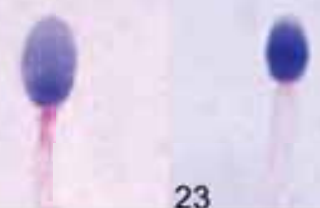

23

24
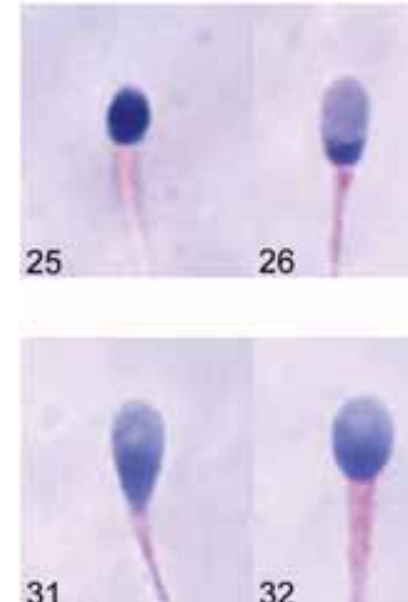

32

33

27

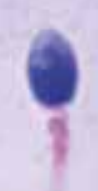

28

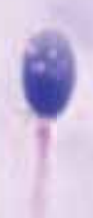

29

30

35

36 
Morphology assessment of spermatozoa in Plate 3

\begin{tabular}{|c|c|c|c|c|c|c|}
\hline Sperm & $\begin{array}{l}\text { Head } \\
\text { shape }\end{array}$ & $\begin{array}{l}\text { Other head } \\
\text { comments }\end{array}$ & $\begin{array}{l}\text { Midpiece } \\
\text { comments }\end{array}$ & $\begin{array}{l}\text { Principal } \\
\text { piece } \\
\text { comments }\end{array}$ & $\begin{array}{l}\text { Overall sperm } \\
\text { classification }\end{array}$ & Comments \\
\hline 1 & abnormal & tapered & thick & & abnormal & \\
\hline 2 & abnormal & & & & abnormal & \\
\hline 3 & abnormal & & irreg & & abnormal & \\
\hline 4 & abnormal & round & & & abnormal & \\
\hline 5 & abnormal & round & & & abnormal & \\
\hline 6 & abnormal & tapered & & & abnormal & \\
\hline 7 & abnormal & tapered & & & abnormal & \\
\hline 8 & abnormal & amorphous & thick & & abnormal & \\
\hline 9 & abnormal & round & thick & & abnormal & \\
\hline 10 & abnormal & tapered & irreg, thick & & abnormal & \\
\hline 11 & - & & & & - & two cells \\
\hline 12 & abnormal & $>2$ vac, $\mathrm{PA}$ vac & & & abnormal & \\
\hline 13 & abnormal & & & & abnormal & \\
\hline 14 & normal & PA vac & & & abnormal & \\
\hline 15 & - & & & & - & pinhead \\
\hline 16 & abnormal & small & & & abnormal & \\
\hline 17 & abnormal & large & & & abnormal & \\
\hline 18 & normal & & thick & & abnormal & \\
\hline 19 & abnormal & & thick & & abnormal & \\
\hline 20 & abnormal & $>2$ vac & insert & & abnormal & \\
\hline 21 & normal & $>70 \%$ acr & & & abnormal & \\
\hline 22 & abnormal & $>70 \%$ acr & & & abnormal & \\
\hline 23 & abnormal & $<40 \%$ acr, small & & & abnormal & \\
\hline 24 & abnormal & $<40 \%$ acr. small & & & abnormal & \\
\hline 25 & abnormal & $<40 \%$ acr, small & & & abnormal & \\
\hline 26 & abnormal & $>70 \%$ acr & & & abnormal & \\
\hline 27 & abnormal & $\begin{array}{c}<40 \% \text { acr, }>2 \\
\text { vac }\end{array}$ & irreg & & abnormal & \\
\hline 28 & normal & $>2$ vac & & & abnormal & \\
\hline 29 & abnormal & tapered & & & abnormal & \\
\hline 30 & abnormal & tapered & & & abnormal & \\
\hline 31 & abnormal & tapered & & & abnormal & \\
\hline 32 & normal & & thick & & abnormal & \\
\hline 33 & normal & & thick & & abnormal & \\
\hline 34 & abnormal & $<40 \%$ acr & thick & & abnormal & \\
\hline 35 & abnormal & & thick, bent & & abnormal & \\
\hline 36 & - & & & & - & pinhead \\
\hline
\end{tabular}




\section{Plate 4}
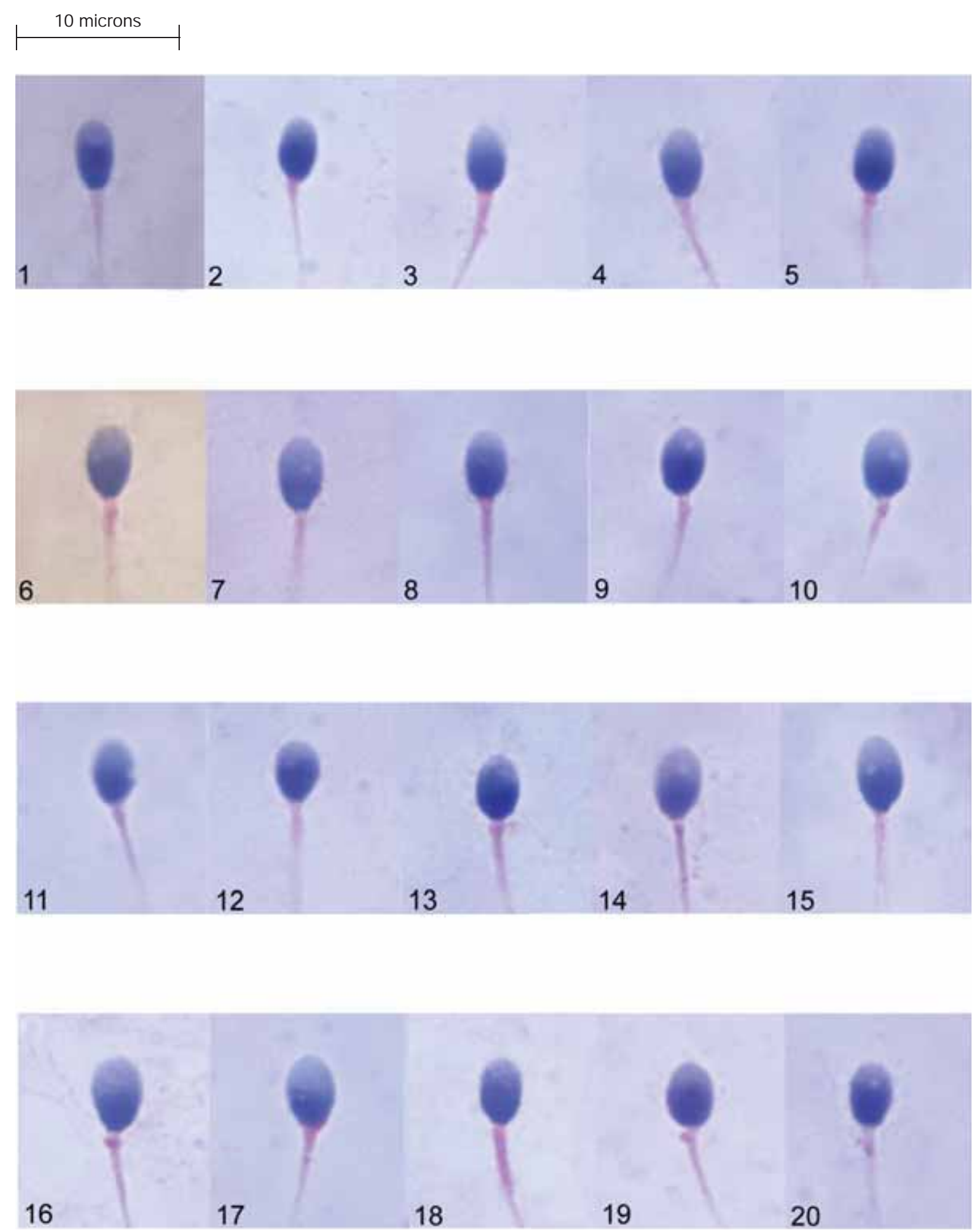

Micrographs courtesy of $C$ Brazil. 
Morphology assessment of spermatozoa in Plate 4

\begin{tabular}{|c|c|c|c|c|c|c|}
\hline Sperm & $\begin{array}{l}\text { Head } \\
\text { shape }\end{array}$ & $\begin{array}{l}\text { Other head } \\
\text { comments }\end{array}$ & $\begin{array}{l}\text { Midpiece } \\
\text { comments }\end{array}$ & $\begin{array}{l}\text { Principal } \\
\text { piece } \\
\text { comments }\end{array}$ & $\begin{array}{l}\text { Overall sperm } \\
\text { classification }\end{array}$ & Comments \\
\hline 1 & abnormal & flat & thick & & abnormal & \\
\hline 2 & normal & & thick, bent & & abnormal & \\
\hline 3 & normal & & thick & & abnormal & \\
\hline 4 & normal & & thick, bent & & abnormal & \\
\hline 5 & normal & & thick & & abnormal & \\
\hline 6 & normal & & thick & & abnormal & \\
\hline 7 & abnormal & irreg & & & abnormal & \\
\hline 8 & normal & & thick & & abnormal & \\
\hline 9 & normal & & insert, bent & & abnormal & \\
\hline 10 & normal & & thick, bent & & abnormal & \\
\hline 11 & abnormal & PA vac & & & abnormal & \\
\hline 12 & abnormal & & & & abnormal & \\
\hline 13 & abnormal & $\begin{array}{c}<40 \% \text { acr } \\
>2 \text { vac }\end{array}$ & thick & & abnormal & \\
\hline 14 & normal & & irreg & & abnormal & \\
\hline 15 & normal & & insert & & abnormal & \\
\hline 16 & normal & & thick & & abnormal & \\
\hline 17 & normal & & insert, thick & & abnormal & \\
\hline 18 & normal & & thick, too long & & abnormal & \\
\hline 19 & normal & $<40 \%$ acr & insert & & abnormal & \\
\hline 20 & normal & $<40 \%$ acr & irreg & & abnormal & \\
\hline
\end{tabular}


Plate 5

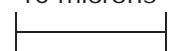

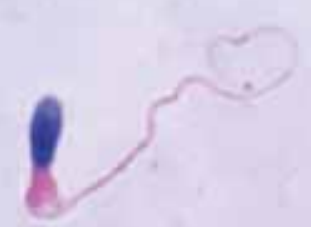

1

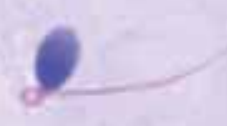

4

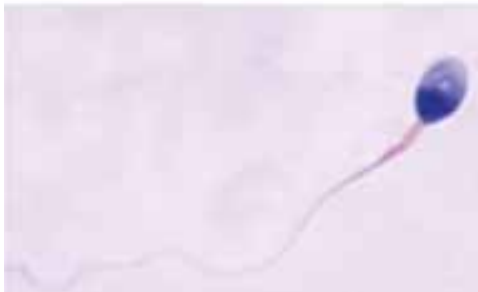

7

8

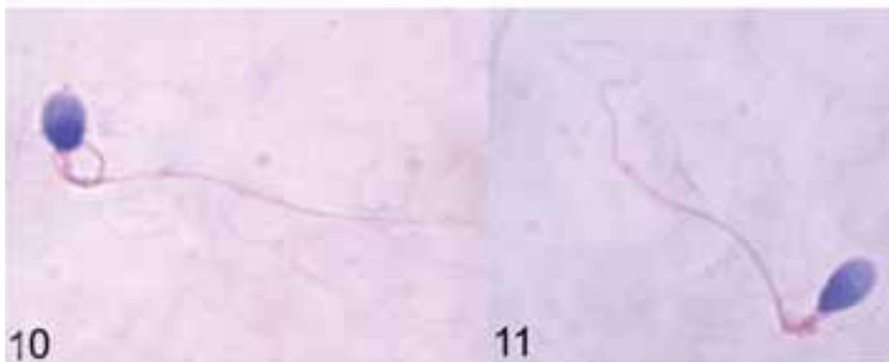

Micrographs courtesy of C Brazil.
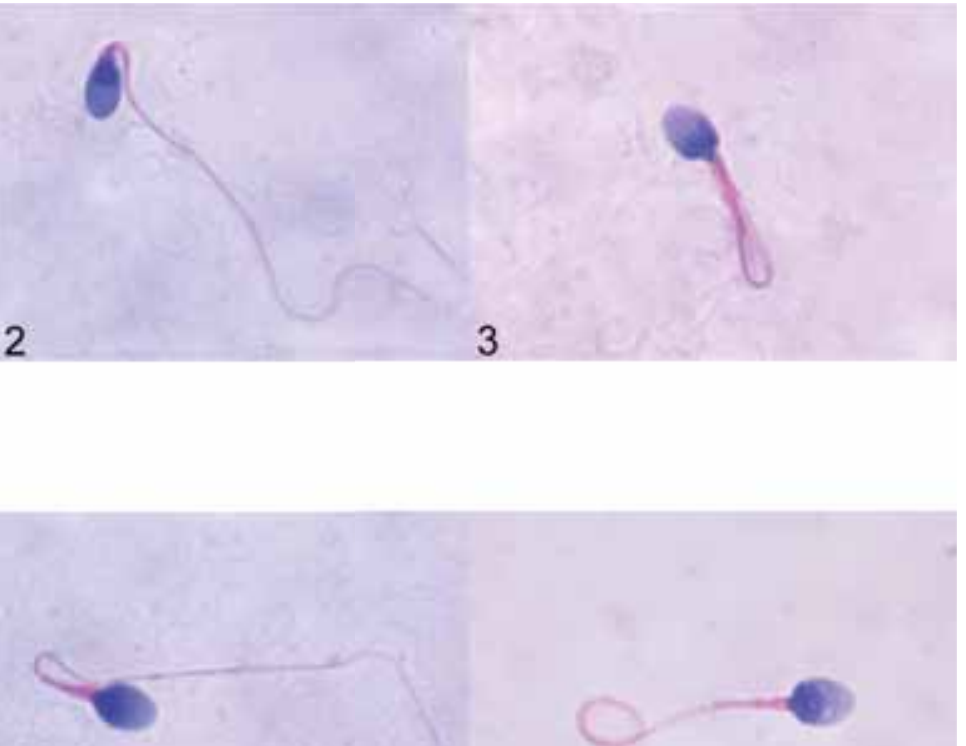

6
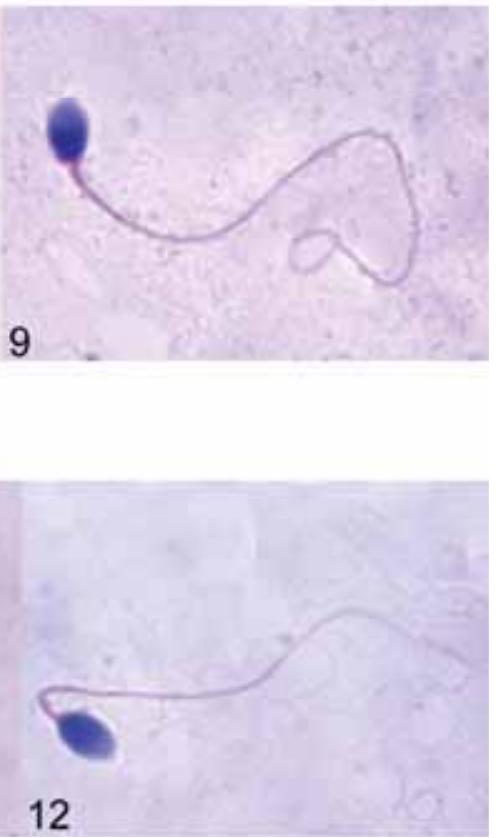

12 
Morphology assessment of spermatozoa in Plate 5

\begin{tabular}{|c|c|c|c|c|c|c|}
\hline Sperm & $\begin{array}{l}\text { Head } \\
\text { shape }\end{array}$ & $\begin{array}{l}\text { Other head } \\
\text { comments }\end{array}$ & $\begin{array}{l}\text { Midpiece } \\
\text { comments }\end{array}$ & $\begin{array}{l}\text { Principal } \\
\text { piece } \\
\text { comments }\end{array}$ & $\begin{array}{l}\text { Overall sperm } \\
\text { classification }\end{array}$ & Comments \\
\hline 1 & abnormal & & ERC & & abnormal & >one third \\
\hline 2 & normal & & bent & normal & abnormal & \\
\hline 3 & abnormal & $>70 \%$ acr & & looped & abnormal & \\
\hline 4 & normal & & bent & normal & abnormal & \\
\hline 5 & normal & & thick & looped & abnormal & \\
\hline 6 & abnormal & PA vac & & coiled & abnormal & \\
\hline 7 & normal & & & & normal & \\
\hline 8 & normal & & & double & abnormal & \\
\hline 9 & abnormal & & & coiled & abnormal & \\
\hline 10 & abnormal & & bent, insert & coiled & abnormal & \\
\hline 11 & normal & & thick & bent & abnormal & \\
\hline 12 & normal & & bent & normal & abnormal & \\
\hline
\end{tabular}




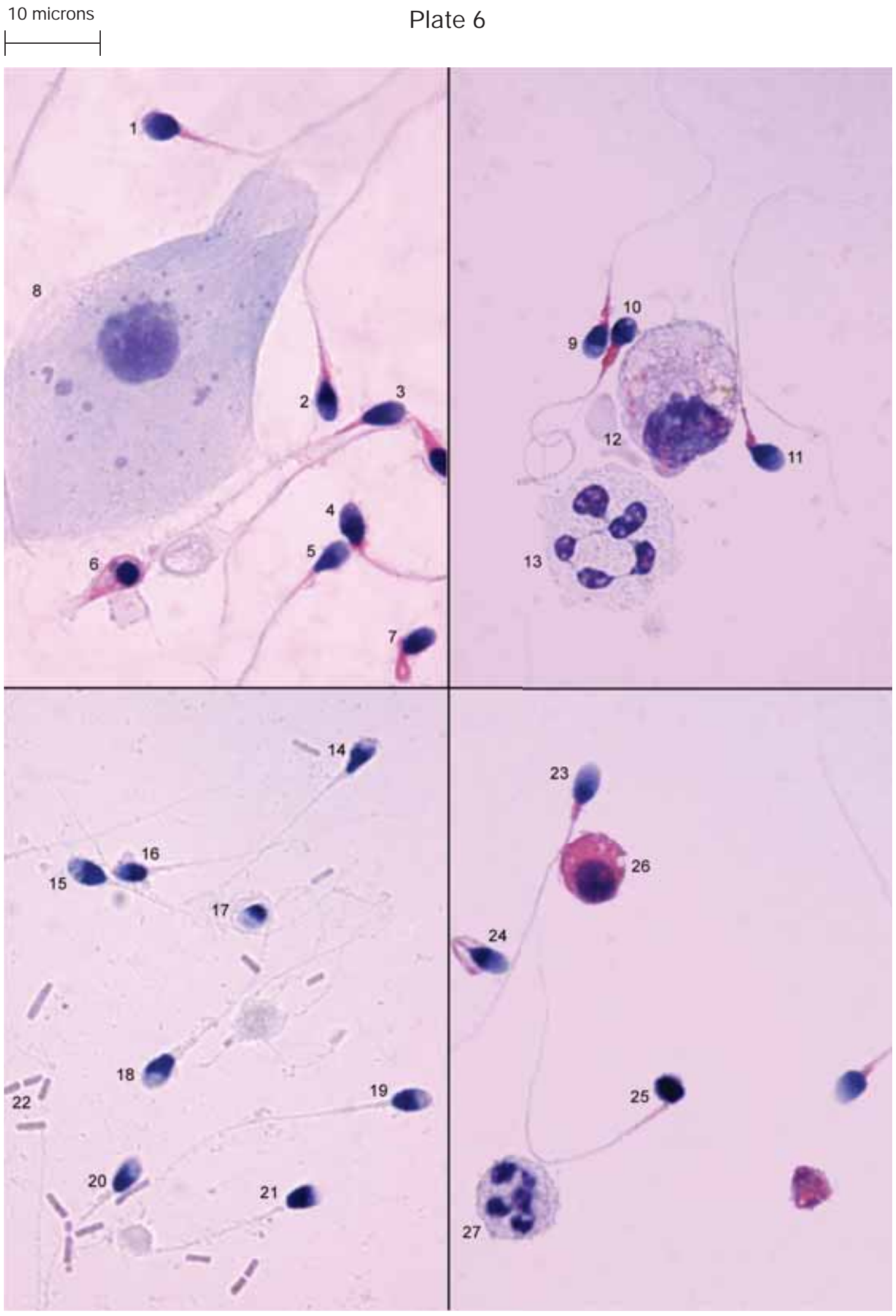

Micrographs courtesy of $C$ Brazil. 
Morphology assessment of spermatozoa in Plate 6

\begin{tabular}{|c|c|c|c|c|c|c|}
\hline Sperm & $\begin{array}{l}\text { Head } \\
\text { shape }\end{array}$ & $\begin{array}{l}\text { Other head } \\
\text { comments }\end{array}$ & $\begin{array}{l}\text { Midpiece } \\
\text { comments }\end{array}$ & $\begin{array}{l}\text { Principal } \\
\text { piece } \\
\text { comments }\end{array}$ & $\begin{array}{l}\text { Overall sperm } \\
\text { classification }\end{array}$ & Comments \\
\hline 1 & normal & $<40 \%$ acr & thick & normal & abnormal & \\
\hline 2 & normal & & thick & & abnormal & \\
\hline 3 & normal & & & & normal & \\
\hline 4 & abnormal & & thick & & abnormal & \\
\hline 5 & abnormal & tapered & & & abnormal & \\
\hline 6 & & & & & not classifiable & $\begin{array}{c}\text { abnormal } \\
\text { spermatozoon }\end{array}$ \\
\hline 7 & abnormal & & thick & coiled & abnormal & \\
\hline 8 & & & & & & epithelial cell \\
\hline 9 & normal & & thick, insert & & abnormal & \\
\hline 10 & abnormal & $<40 \%$ acr & thick & & abnormal & \\
\hline 11 & normal & & thick & & abnormal & \\
\hline 12 & & & & & & $\begin{array}{l}\text { degenerating } \\
\text { macrophage? }\end{array}$ \\
\hline 13 & & & & & & polymorph \\
\hline 14 & abnormal & pyriform & & & abnormal & \\
\hline 15 & normal & & & & normal & \\
\hline 16 & abnormal & $<40 \%$ acr & & & abnormal & \\
\hline 17 & abnormal & round & & not seen & abnormal & free head? \\
\hline 18 & abnormal & & thick & & abnormal & \\
\hline 19 & normal & & & & normal & \\
\hline 20 & normal & & & & normal & If PP OK \\
\hline 21 & abnormal & flat & & & abnormal & \\
\hline 22 & & & & & & bacilli \\
\hline 23 & normal & & thick & & abnormal & \\
\hline 24 & normal & & thick & coiled & abnormal & \\
\hline 25 & abnormal & amorphous & & & abnormal & \\
\hline 26 & & & & & & spermatid \\
\hline 27 & & & & & & polymorph \\
\hline
\end{tabular}


10 microns

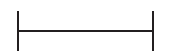

Plate 7

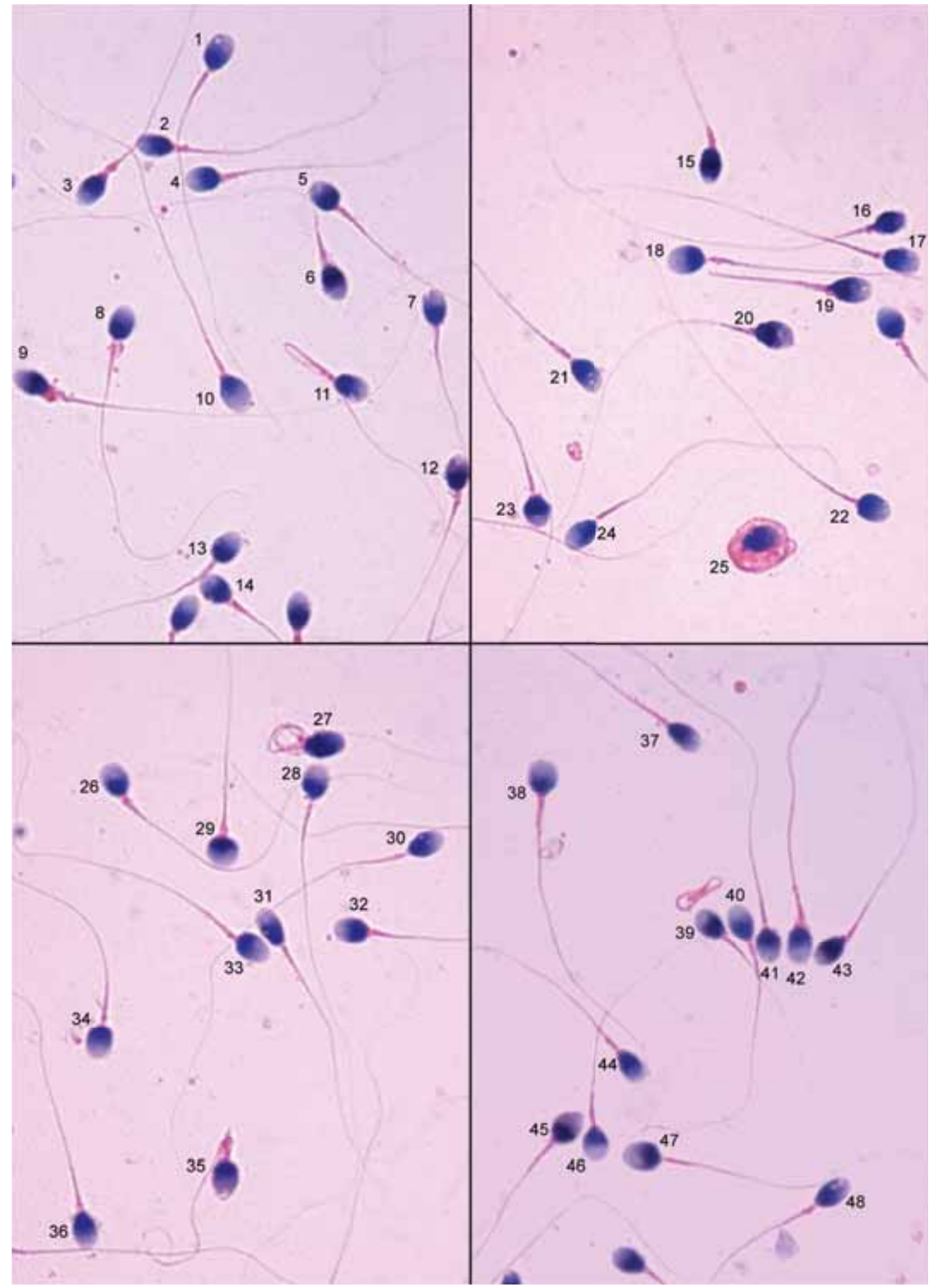

Micrographs courtesy of C B razil. 
Morphology assessment of spermatozoa in Plate 7

\begin{tabular}{|c|c|c|c|c|c|c|}
\hline Sperm & $\begin{array}{l}\text { Head } \\
\text { shape }\end{array}$ & $\begin{array}{l}\text { Other head } \\
\text { comments }\end{array}$ & $\begin{array}{l}\text { Midpiece } \\
\text { comments }\end{array}$ & $\begin{array}{l}\text { Principal } \\
\text { piece } \\
\text { comments }\end{array}$ & $\begin{array}{l}\text { Overall sperm } \\
\text { classification }\end{array}$ & Comments \\
\hline 1 & normal & $2 \mathrm{vac}$ & & & normal & \\
\hline 2 & normal & & & & normal & \\
\hline 3 & normal & & thick & & abnormal & \\
\hline 4 & normal & & & & normal & \\
\hline 5 & normal & & & & normal & if $P P O K$ \\
\hline 6 & normal & & thick & & abnormal & \\
\hline 7 & normal & vac on surface & & & normal & \\
\hline 8 & normal & & $C D$ & & normal & $<0$ ne third \\
\hline 9 & abnormal & & thick, ERC & & abnormal & >one third \\
\hline 10 & normal & & & & normal & \\
\hline 11 & normal & PA vac & & looped & abnormal & \\
\hline 12 & normal & & & & normal & if $P P O K$ \\
\hline 13 & normal & PA vac & & & abnormal & \\
\hline 14 & normal & PA vac & & & abnormal & \\
\hline 15 & abnormal & $<40 \%$ acr & thick & & abnormal & \\
\hline 16 & abnormal & $<40 \%$ acr & & & abnormal & \\
\hline 17 & normal & & & & normal & \\
\hline 18 & normal & & & & normal & if $P P O K$ \\
\hline 19 & normal & & thick & short & abnormal & \\
\hline 20 & abnormal & & thick & & abnormal & \\
\hline 21 & normal & $>2 \mathrm{vac}$ & & & abnormal & \\
\hline 22 & abnormal & round & & & abnormal & \\
\hline 23 & abnormal & round & & & abnormal & \\
\hline 24 & normal & & & & normal & \\
\hline 25 & & & & & & $\begin{array}{l}\text { sperm head in } \\
\text { cytoplasm? }\end{array}$ \\
\hline 26 & normal & & & & normal & \\
\hline 27 & normal & no acro & & coiled & abnormal & \\
\hline 28 & normal & & & & normal & \\
\hline 29 & abnormal & round & & & abnormal & \\
\hline 30 & normal & PA vac & & & abnormal & \\
\hline 31 & abnormal & $\begin{array}{c}\text { tapered, } \\
\text { PA vac }\end{array}$ & & & abnormal & \\
\hline 32 & normal & & & & normal & if $P P O K$ \\
\hline 33 & normal & & & & normal & \\
\hline 34 & normal & & & & normal & If PP OK \\
\hline 35 & abnormal & & thick & bent & abnormal & \\
\hline 36 & normal & & & & normal & if $P P O K$ \\
\hline 37 & normal & & & & normal & if PP OK \\
\hline 38 & abnormal & round & & & abnormal & \\
\hline 39 & normal & & & & normal & \\
\hline 40 & normal & & & & normal & \\
\hline 41 & normal & & & & normal & \\
\hline 42 & normal & & thick & & abnormal & \\
\hline 43 & normal & $<40 \%$ acr & & & abnormal & \\
\hline 44 & & out of focus & & & & $\begin{array}{c}\text { not } \\
\text { assessed }\end{array}$ \\
\hline 45 & abnormal & round & & & abnormal & \\
\hline 46 & abnormal & round & & & abnormal & \\
\hline 47 & normal & & & & normal & \\
\hline 48 & normal & & & & normal & if PP OK \\
\hline
\end{tabular}


10 microns

1
Plate 8

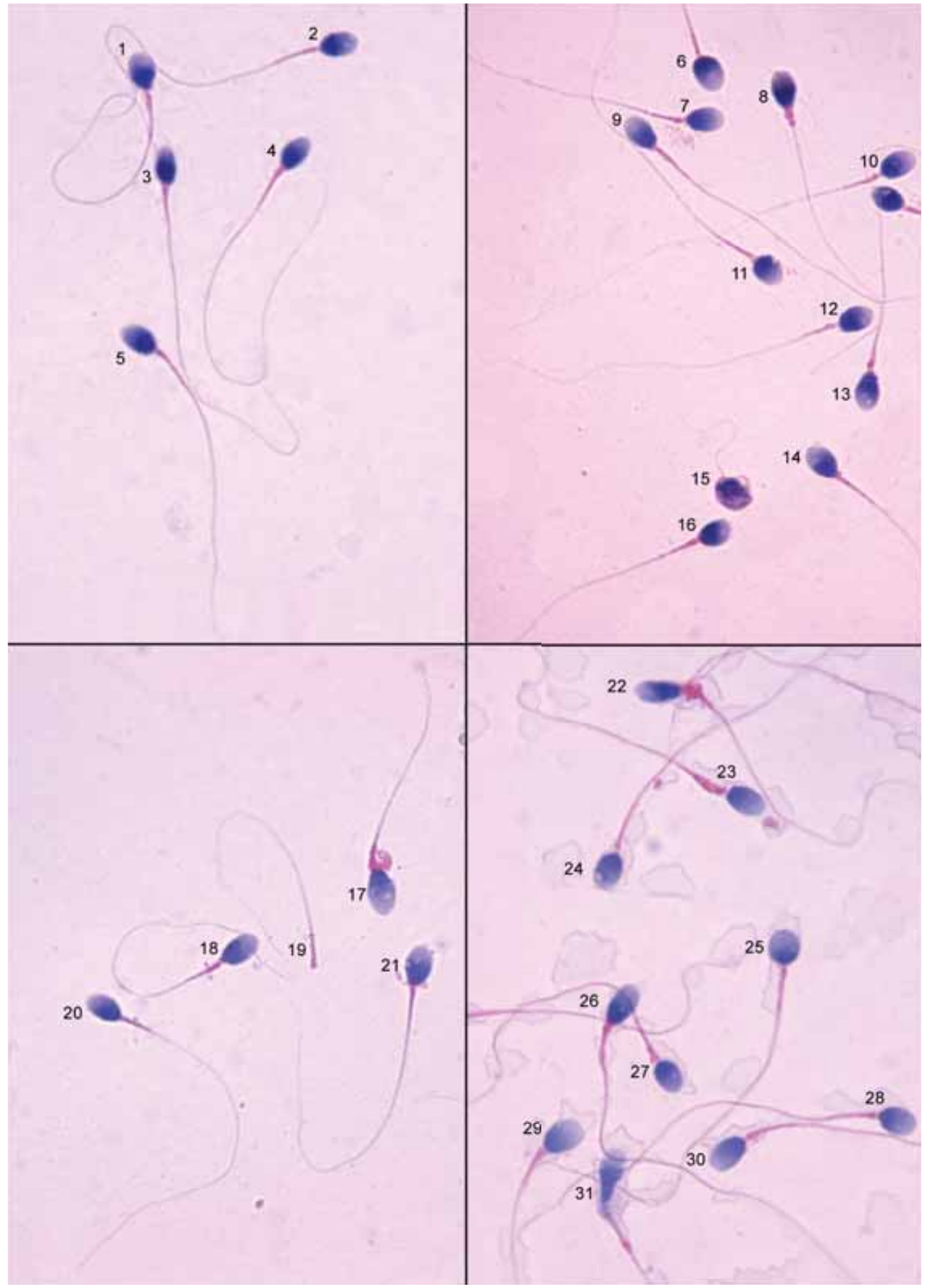


Morphology assessment of spermatozoa in Plate 8

\begin{tabular}{|c|c|c|c|c|c|c|}
\hline Sperm & $\begin{array}{l}\text { Head } \\
\text { shape }\end{array}$ & $\begin{array}{l}\text { Other head } \\
\text { comments }\end{array}$ & $\begin{array}{l}\text { Midpiece } \\
\text { comments }\end{array}$ & $\begin{array}{l}\text { Principal } \\
\text { piece } \\
\text { comments }\end{array}$ & $\begin{array}{l}\text { Overall sperm } \\
\text { classification }\end{array}$ & Comments \\
\hline 1 & normal & & & normal & normal & \\
\hline 2 & normal & $>2$ vac & & normal & abnormal & \\
\hline 3 & abnormal & tapered & & & abnormal & \\
\hline 4 & normal & & & normal & normal & \\
\hline 5 & normal & & & & normal & \\
\hline 6 & normal & & & & normal & if $P P O K$ \\
\hline 7 & normal & & & & normal & if $P P O K$ \\
\hline 8 & normal & & thick & & abnormal & \\
\hline 9 & normal & & & & normal & \\
\hline 10 & normal & & & & normal & \\
\hline 11 & normal & PA vac & & & abnormal & \\
\hline 12 & normal & & & & normal & \\
\hline 13 & abnormal & & & & abnormal & \\
\hline 14 & normal & & & & normal & if PP OK \\
\hline 15 & abnormal & amorphous & & defect & abnormal & \\
\hline 16 & normal & & & & normal & If PP OK \\
\hline 17 & abnormal & $>70 \%$ acr & thick, ERC & & abnormal & >one third \\
\hline 18 & normal & & & & normal & \\
\hline 19 & & & & & & pinhead \\
\hline 20 & normal & & & & normal & \\
\hline 21 & normal & PA vac & & & abnormal & \\
\hline 22 & abnormal & tapered & thick, ERC & & abnormal & >one third \\
\hline 23 & abnormal & flat & thick & & abnormal & \\
\hline 24 & normal & $>2$ vac & & & abnormal & \\
\hline 25 & abnormal & round & & & abnormal & \\
\hline 26 & normal & & thick & & abnormal & \\
\hline 27 & normal & & thick & & abnormal & \\
\hline 28 & normal & $\begin{array}{c}>2 \mathrm{vac} \\
>70 \% \text { acr }\end{array}$ & & & abnormal & \\
\hline 29 & abnormal & & & & abnormal & \\
\hline 30 & normal & $>70 \%$ acr & & & abnormal & \\
\hline 31 & abnormal & pyriform & & & abnormal & \\
\hline
\end{tabular}


10 microns

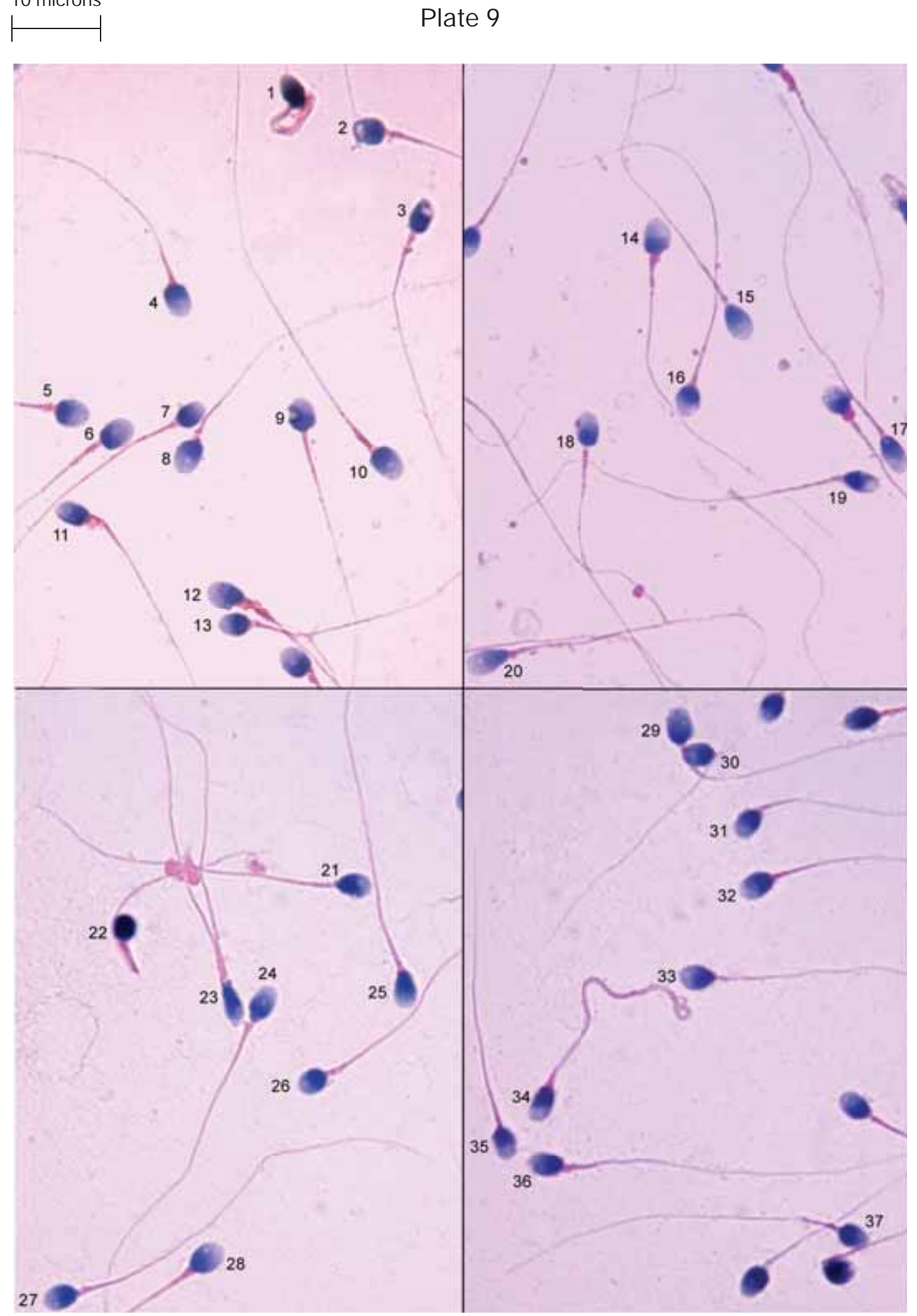

Micrographs courtesy of C Brazil. 
Morphology assessment of spermatozoa in Plate 9

\begin{tabular}{|c|c|c|c|c|c|c|}
\hline Sperm & $\begin{array}{l}\text { Head } \\
\text { shape }\end{array}$ & $\begin{array}{l}\text { Other head } \\
\text { comments }\end{array}$ & $\begin{array}{l}\text { Midpiece } \\
\text { comments }\end{array}$ & $\begin{array}{l}\text { Principal } \\
\text { piece } \\
\text { comments }\end{array}$ & $\begin{array}{l}\text { Overall sperm } \\
\text { classification }\end{array}$ & Comments \\
\hline 1 & abnormal & & & coiled & abnormal & \\
\hline 2 & & overlapping & & & & not assessed \\
\hline 3 & abnormal & $<40 \%$ acr & & & abnormal & \\
\hline 4 & normal & & & & normal & if PP OK \\
\hline 5 & normal & & & & normal & if PP OK \\
\hline 6 & normal & $>70 \%$ acr & insert & & abnormal & \\
\hline 7 & normal & & insert & & abnormal & \\
\hline 8 & normal & $>70 \%$ acr & insert & & abnormal & \\
\hline 9 & abnormal & PA vac & & & abnormal & \\
\hline 10 & normal & $>2$ vac & thick & & abnormal & \\
\hline 11 & abnormal & & thick, ERC & & abnormal & >one third \\
\hline 12 & abnormal & & $\begin{array}{l}\text { thick, insert, } \\
\text { ERC }\end{array}$ & & abnormal & >one third \\
\hline 13 & normal & & & & normal & if $P P O K$ \\
\hline 14 & abnormal & & thick & & abnormal & \\
\hline 15 & normal & & & normal & normal & \\
\hline 16 & abnormal & & & & abnormal & \\
\hline 17 & abnormal & $\begin{array}{c}\text { tapered, } 3 \text { vac, } \\
\text { PA vac }\end{array}$ & & & abnormal & \\
\hline 18 & normal & & & & normal & \\
\hline 19 & abnormal & vac $>20 \%$ & & & abnormal & \\
\hline 20 & abnormal & tapered & & & abnormal & \\
\hline 21 & normal & PA vac & & & abnormal & \\
\hline 22 & abnormal & amorphous & & bent & abnormal & \\
\hline 23 & abnormal & tapered & & double & abnormal & \\
\hline 24 & abnormal & PA vac & & & abnormal & \\
\hline 25 & normal & $>2$ vac & & & abnormal & \\
\hline 26 & normal & & & & normal & if $P P O K$ \\
\hline 27 & normal & & & & normal & \\
\hline 28 & normal & & & & normal & if PP OK \\
\hline 29 & & overlapping & & & & not assessed \\
\hline 30 & & overlapping & & & & not assessed \\
\hline 31 & normal & & & & normal & if PP OK \\
\hline 32 & normal & & & & normal & if PP OK \\
\hline 33 & normal & & & & normal & if PP OK \\
\hline 34 & normal & & thick & thick, coiled & abnormal & \\
\hline 35 & abnormal & 1 side not oval & & & abnormal & \\
\hline 36 & normal & $<40 \%$ acr & & & abnormal & \\
\hline 37 & & overlapping & & & & not assessed \\
\hline
\end{tabular}




\section{0 microns}

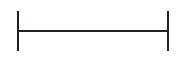

Plate 10

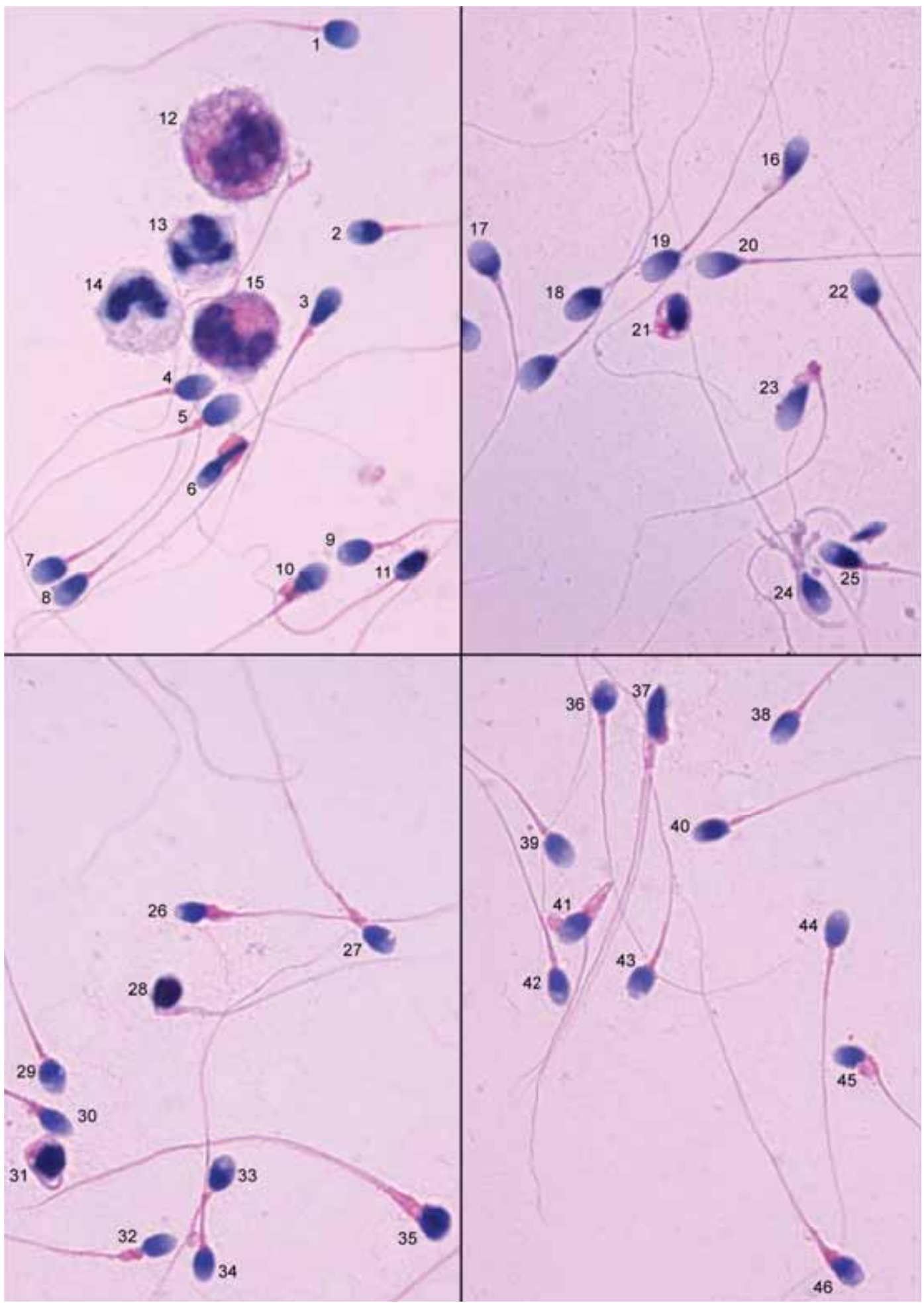

Micrographs courtesy of $C$ Brazil. 
Morphology assessment of spermatozoa in Plate 10

\begin{tabular}{|c|c|c|c|c|c|c|}
\hline Sperm & $\begin{array}{l}\text { Head } \\
\text { shape }\end{array}$ & $\begin{array}{l}\text { Other head } \\
\text { comments }\end{array}$ & $\begin{array}{l}\text { Midpiece } \\
\text { comments }\end{array}$ & $\begin{array}{l}\text { Principal } \\
\text { piece } \\
\text { comments }\end{array}$ & $\begin{array}{l}\text { Overall sperm } \\
\text { classification }\end{array}$ & Comments \\
\hline 1 & normal & & insert & & abnormal & \\
\hline 2 & normal & & & & normal & if $P P O K$ \\
\hline 3 & abnormal & pyriform & & & abnormal & \\
\hline 4 & normal & & & & normal & \\
\hline 5 & normal & & thick & & abnormal & \\
\hline 6 & abnormal & pyriform & ERC & bent & abnormal & >one third \\
\hline 7 & normal & & & & normal & \\
\hline 8 & normal & & & & normal & \\
\hline 9 & normal & 3 vac & & & abnormal & \\
\hline 10 & abnormal & tapered & thick, ERC & & abnormal & >one third \\
\hline 11 & abnormal & $\begin{array}{l}\text { tapered, } \\
<40 \% \mathrm{acr}\end{array}$ & & bent & abnormal & \\
\hline 12 & & & & & & monocyte \\
\hline 13 & & & & & & polymorph \\
\hline 14 & & & & & & polymorph \\
\hline 15 & & & & & & monocyte \\
\hline 16 & abnormal & tapered & & & abnormal & \\
\hline 17 & normal & & & & normal & if PP OK \\
\hline 18 & normal & & & & normal & \\
\hline 19 & normal & & & & normal & \\
\hline 20 & normal & & & & normal & if $P P O K$ \\
\hline 21 & abnormal & amorphous & & & abnormal & \\
\hline 22 & normal & & & & normal & if $P P O K$ \\
\hline 23 & abnormal & tapered & thick & bent & abnormal & \\
\hline 24 & & overlapping & & & & not assessed \\
\hline 25 & abnormal & tapered & & & abnormal & \\
\hline 26 & abnormal & amorphous & thick, ERC & & abnormal & >one third \\
\hline 27 & normal & & thick & & abnormal & \\
\hline 28 & abnormal & amorphous & thick & & abnormal & \\
\hline 29 & abnormal & PA vac & & & abnormal & \\
\hline 30 & abnormal & & thick & & abnormal & \\
\hline 31 & abnormal & & thick & coiled & abnormal & \\
\hline 32 & normal & & thick & & abnormal & \\
\hline 33 & & overlapping & & & & not assessed \\
\hline 34 & & overlapping & & & & not assessed \\
\hline 35 & abnormal & $\begin{array}{c}\text { amorphous, } \\
\text { no acro }\end{array}$ & thick & & abnormal & \\
\hline 36 & normal & $<40 \%$ acr & & & abnormal & \\
\hline 37 & abnormal & pyriform & thick & double & abnormal & \\
\hline 38 & normal & & & & normal & if $\mathrm{PP} O K$ \\
\hline 39 & normal & & thick & & abnormal & \\
\hline 40 & abnormal & $<40 \%$ acr & & & abnormal & \\
\hline 41 & abnormal & & thick & bent & abnormal & \\
\hline 42 & normal & & & & normal & if $\mathrm{PP} O K$ \\
\hline 43 & normal & $\begin{array}{c}2 \mathrm{vac} \\
<40 \% \text { acr }\end{array}$ & & & abnormal & \\
\hline 44 & normal & & & & normal & \\
\hline 45 & abnormal & & thick, ECR & & abnormal & >one third \\
\hline 46 & abnormal & & thick & & abnormal & \\
\hline
\end{tabular}



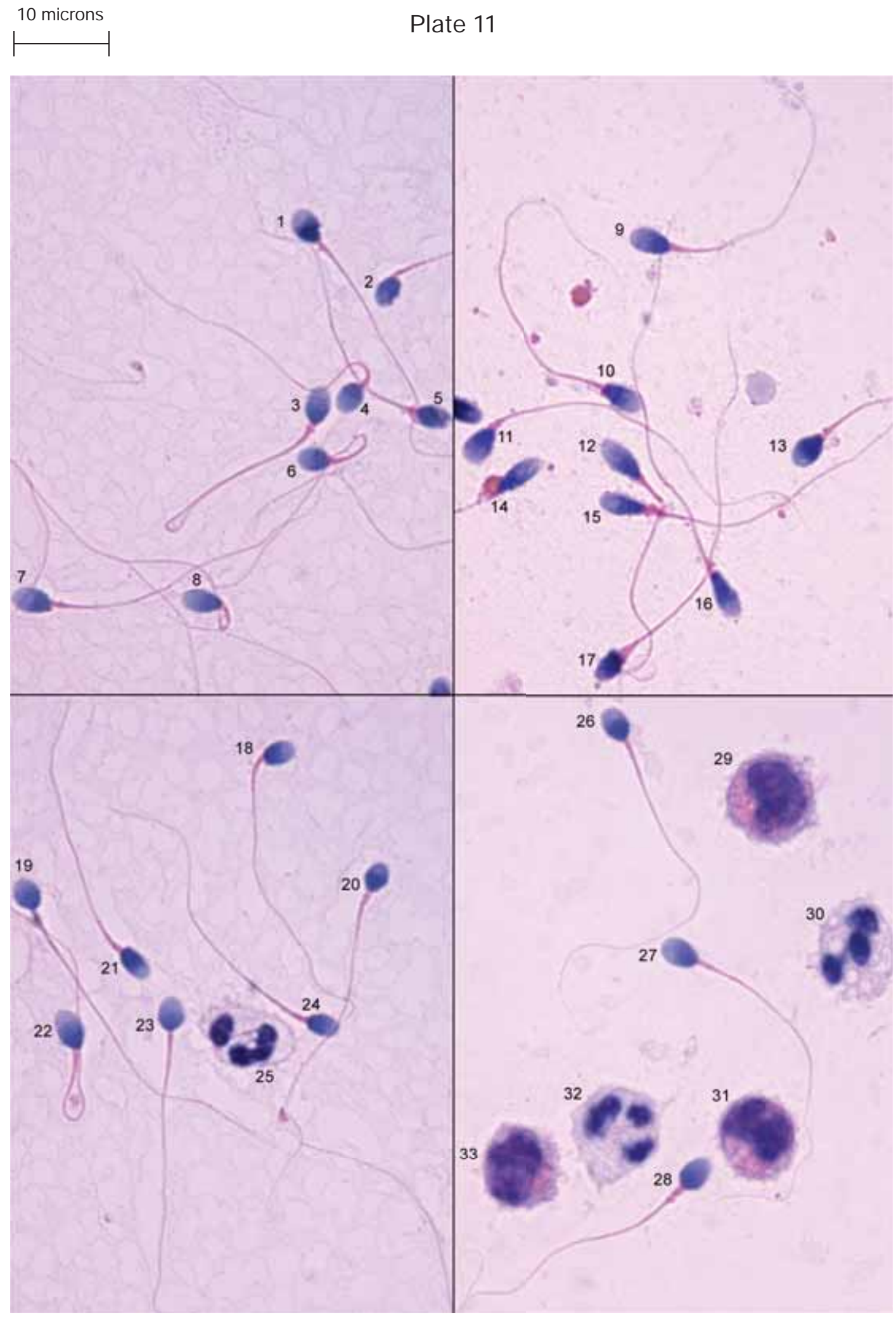
Morphology assessment of spermatozoa in Plate 11

\begin{tabular}{|c|c|c|c|c|c|c|}
\hline Sperm & $\begin{array}{l}\text { Head } \\
\text { shape }\end{array}$ & $\begin{array}{l}\text { Other head } \\
\text { comments }\end{array}$ & $\begin{array}{l}\text { Midpiece } \\
\text { comments }\end{array}$ & $\begin{array}{l}\text { Principal } \\
\text { piece } \\
\text { comments }\end{array}$ & $\begin{array}{l}\text { Overall sperm } \\
\text { classification }\end{array}$ & Comments \\
\hline 1 & abnormal & & insert & & abnormal & \\
\hline 2 & abnormal & & insert & & abnormal & \\
\hline 3 & normal & & thick & looped & abnormal & \\
\hline 4 & normal & & & & normal & \\
\hline 5 & abnormal & $\begin{array}{l}>2 \mathrm{vac} \\
<40 \% \text { acr }\end{array}$ & thick & & abnormal & \\
\hline 6 & normal & & & looped & abnormal & \\
\hline 7 & abnormal & & insert & & abnormal & \\
\hline 8 & normal & & & looped & abnormal & \\
\hline 9 & abnormal & $\begin{array}{l}>70 \% \text { acr } \\
\text { tapered }\end{array}$ & & & abnormal & \\
\hline 10 & abnormal & tapered & & & abnormal & \\
\hline 11 & normal & & thick & & abnormal & \\
\hline 12 & abnormal & tapered & & & abnormal & \\
\hline 13 & normal & $<40 \%$ acr & thick & & abnormal & \\
\hline 14 & abnormal & tapered & thick, ERC & & abnormal & >one third \\
\hline 15 & abnormal & tapered & thick & & abnormal & \\
\hline 16 & abnormal & tapered & & & abnormal & \\
\hline 17 & abnormal & amorphous & thick & & abnormal & \\
\hline 18 & normal & & & & normal & \\
\hline 19 & normal & & & & abnormal & \\
\hline 20 & abnormal & & & & abnormal & \\
\hline 21 & abnormal & & & & abnormal & \\
\hline 22 & normal & $>70 \%$ acr & & looped & abnormal & \\
\hline 23 & normal & & & & normal & \\
\hline 24 & normal & & & & normal & \\
\hline 25 & & & & & & polymorph \\
\hline 26 & normal & & & & normal & \\
\hline 27 & normal & & & & normal & \\
\hline 28 & normal & $>70 \%$ acr & & & abnormal & \\
\hline 29 & & & & & & monocyte \\
\hline 30 & & & & & & polymorph \\
\hline 31 & & & & & & monocyte \\
\hline 32 & & & & & & polymorph \\
\hline 33 & & & & & & monocyte \\
\hline
\end{tabular}




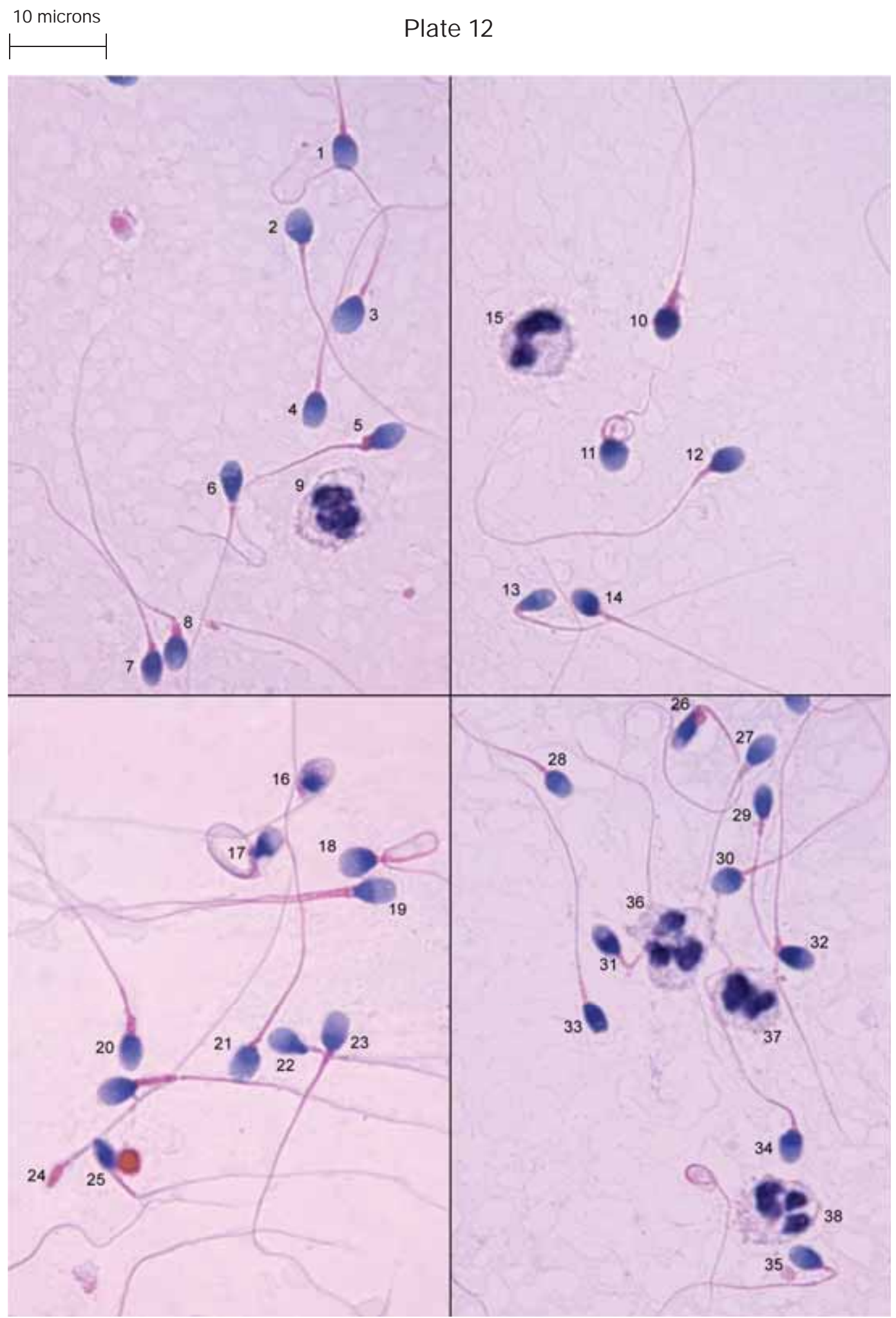

Micrographs courtesy of C Brazil. 
Morphology assessment of spermatozoa in Plate 12

\begin{tabular}{|c|c|c|c|c|c|c|}
\hline Sperm & $\begin{array}{l}\text { Head } \\
\text { shape }\end{array}$ & $\begin{array}{l}\text { Other head } \\
\text { comments }\end{array}$ & $\begin{array}{l}\text { Midpiece } \\
\text { comments }\end{array}$ & $\begin{array}{l}\text { Principal } \\
\text { piece } \\
\text { comments }\end{array}$ & $\begin{array}{l}\text { Overall sperm } \\
\text { classification }\end{array}$ & Comments \\
\hline 1 & normal & $>70 \%$ acr & & & abnormal & \\
\hline 2 & abnormal & & & & abnormal & \\
\hline 3 & abnormal & $>70 \%$ acr & & & abnormal & \\
\hline 4 & normal & & & & normal & if $P P O K$ \\
\hline 5 & abnormal & & thick & & abnormal & \\
\hline 6 & abnormal & tapered & & & abnormal & \\
\hline 7 & & not in focus & thick & & & not assessed \\
\hline 8 & abnormal & & thick, bent & & abnormal & \\
\hline 9 & & & & & & $\begin{array}{l}\text { degenerating } \\
\text { leukocyte }\end{array}$ \\
\hline 10 & abnormal & & thick & & abnormal & \\
\hline 11 & abnormal & round & & coiled & abnormal & \\
\hline 12 & normal & & & & normal & \\
\hline 13 & abnormal & tapered & bent & & abnormal & \\
\hline 14 & abnormal & & insert & & abnormal & \\
\hline 15 & & & & & & polymorph \\
\hline 16 & abnormal & amorphous & & & abnormal & \\
\hline 17 & abnormal & & & coiled & abnormal & \\
\hline 18 & abnormal & & thick & coiled & abnormal & \\
\hline 19 & normal & & & double & abnormal & \\
\hline 20 & abnormal & & thick & & abnormal & \\
\hline 21 & & overlapping & & & & not assessed \\
\hline 22 & abnormal & pyriform & & & abnormal & \\
\hline 23 & normal & & & & normal & \\
\hline 24 & abnormal & & & & abnormal & pinhead \\
\hline 25 & abnormal & amorphous & & bent & abnormal & \\
\hline 26 & abnormal & amorphous & thick, bent & & abnormal & \\
\hline 27 & normal & & thick & & abnormal & \\
\hline 28 & normal & & & & normal & if $\mathrm{PP} O K$ \\
\hline 29 & abnormal & tapered & & & abnormal & \\
\hline 30 & abnormal & round & & & abnormal & \\
\hline 31 & normal & & bent & overlap & & not assessed \\
\hline 32 & normal & & thick, bent & & abnormal & \\
\hline 33 & abnormal & & & & abnormal & \\
\hline 34 & abnormal & & & & abnormal & \\
\hline 35 & normal & & bent & & abnormal & \\
\hline 36 & & & & & & polymorph \\
\hline 37 & & & & & & polymorph \\
\hline 38 & & & & & & polymorph \\
\hline
\end{tabular}


15 microns

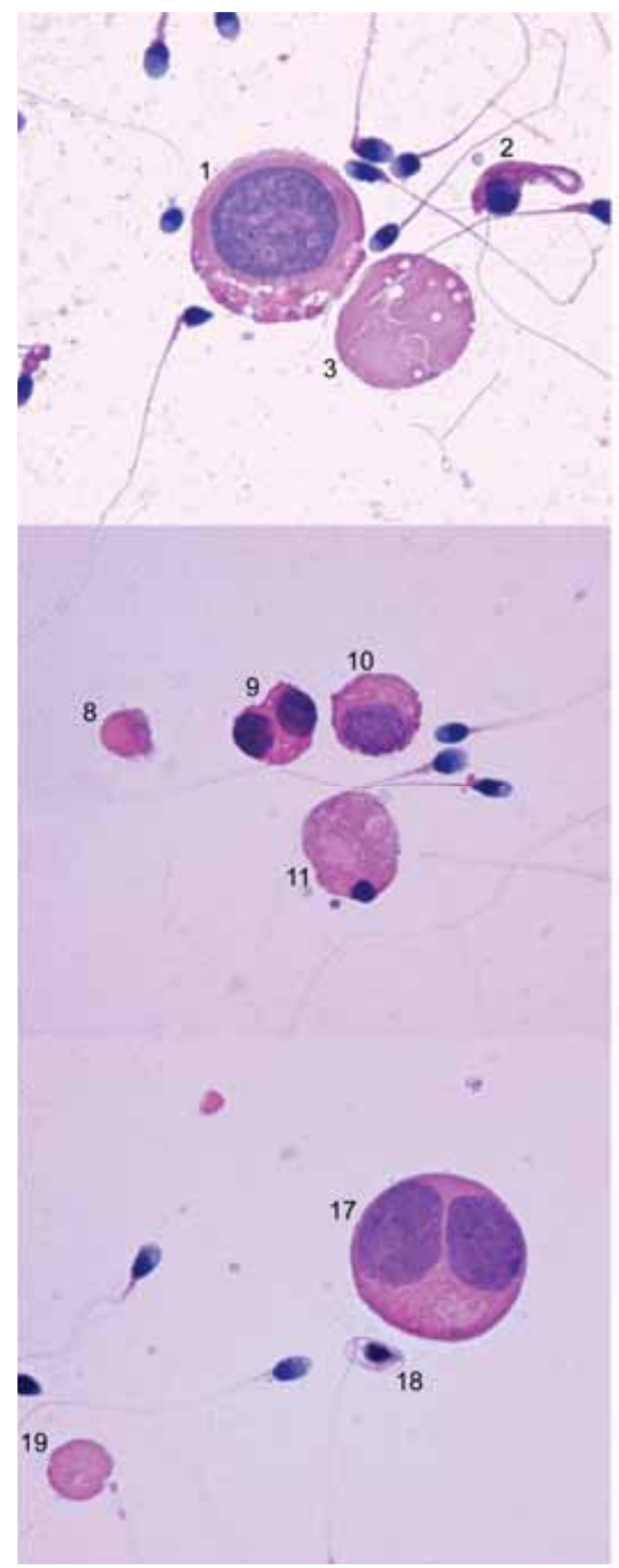

Micrographs courtesy of C Brazil.
Plate 13

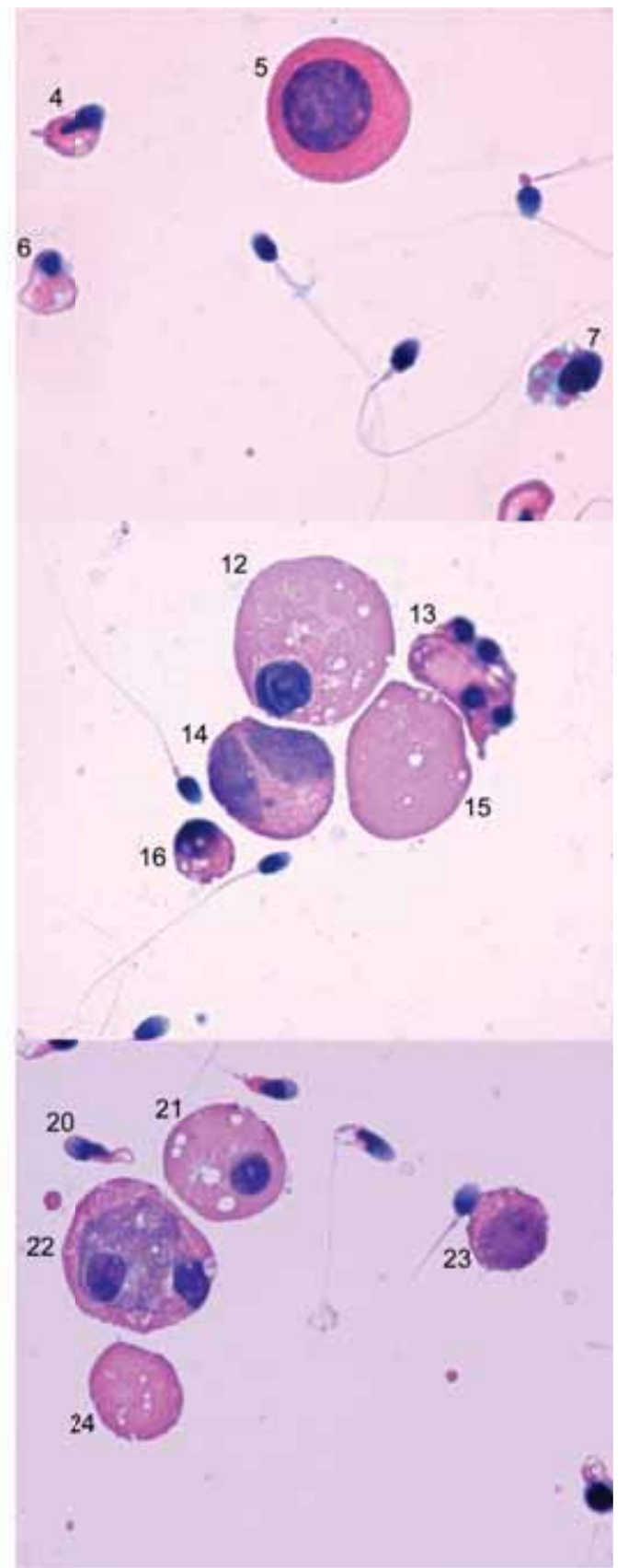


Assessment of cells in Plate 13

\begin{tabular}{|r|l|}
\hline Cell & Cell type \\
\hline 1 & macrophage \\
\hline 2 & abnormal spermatozoon \\
\hline 3 & cytoplasm \\
\hline 4 & abnormal spermatozoon \\
\hline 5 & spermatocyte \\
\hline 6 & abnormal spermatozoon \\
\hline 7 & abnormal spermatozoon? Loose head on cytoplasm? \\
\hline 8 & cytoplasm \\
\hline 9 & dividing spermatid \\
\hline 10 & spermatocyte \\
\hline 11 & degenerating spermatid \\
\hline 12 & spermatid \\
\hline 13 & degenerating spermatid \\
\hline 14 & dividing spermatocyte \\
\hline 15 & cytoplasm \\
\hline 16 & degenerating spermatid \\
\hline 17 & dividing spermatocyte \\
\hline 18 & abnormal spermatozoon \\
\hline 19 & cytoplasm \\
\hline 20 & abnormal spermatozoon \\
\hline 21 & spermatid \\
\hline 22 & phagocytosing macrophage \\
\hline 23 & spermatocyte \\
\hline 24 & cytoplasm \\
\hline
\end{tabular}


15 microns

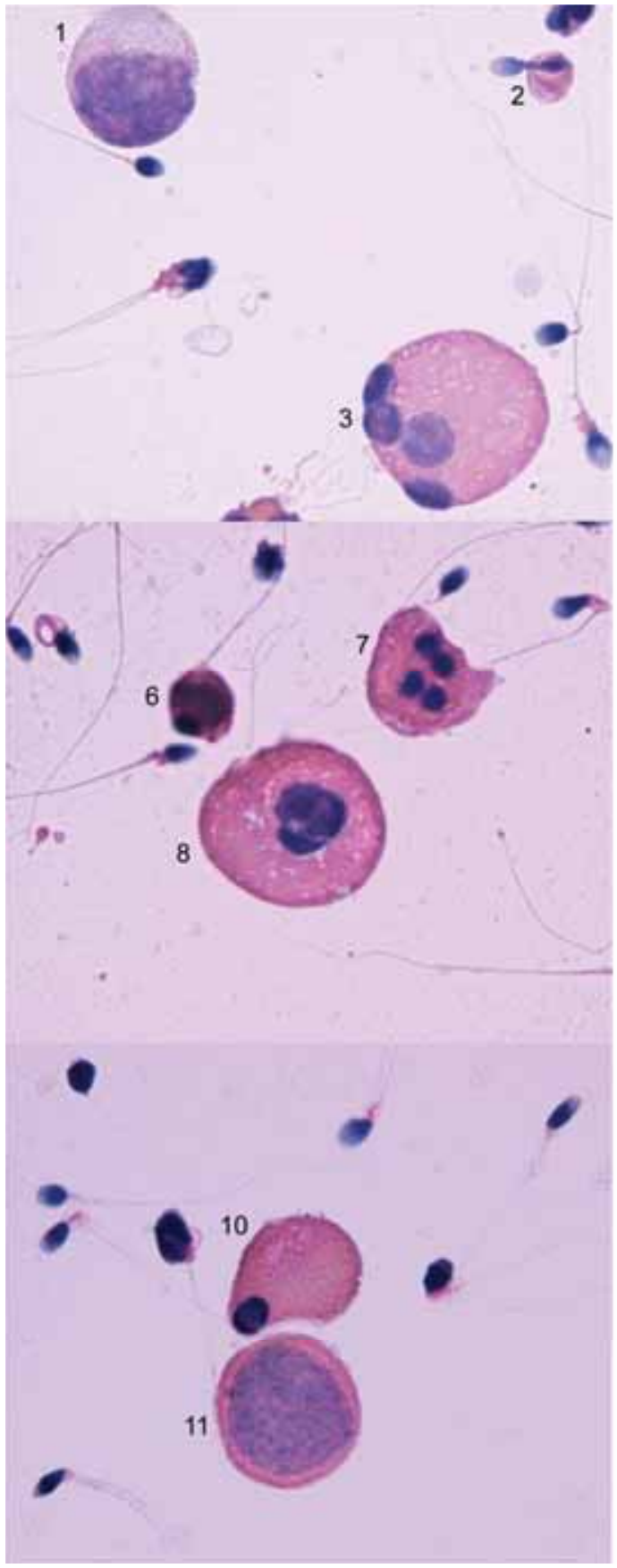

Plate 14

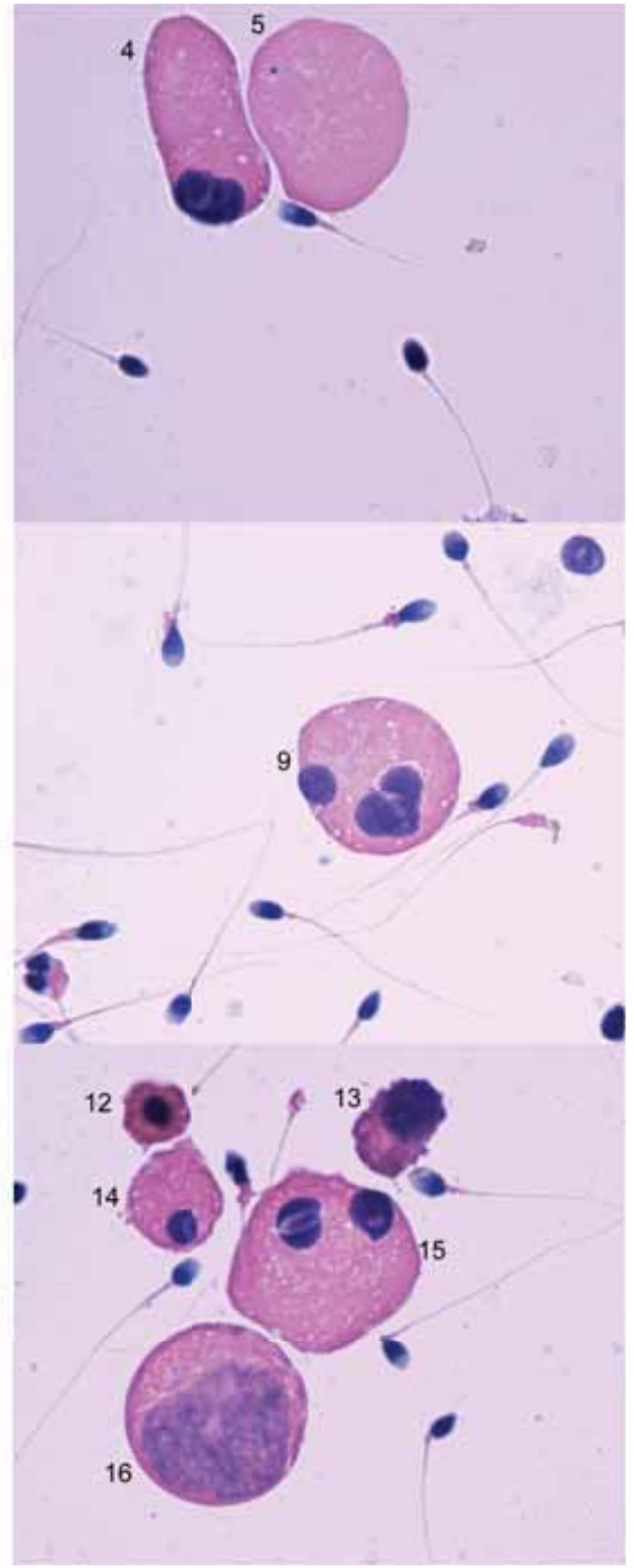

Micrographs courtesy of C Brazil. 
Assessment of cells in Plate 14

\begin{tabular}{|r|l|}
\hline \multicolumn{1}{|r|}{ Cell } & Cell type \\
\hline 1 & macrophage \\
\hline 2 & abnormal spermatozoon \\
\hline 3 & (dividing) spermatid \\
\hline 4 & (dividing) spermatid \\
\hline 5 & cytoplasm \\
\hline 6 & not classifiable \\
\hline 7 & degenerating spermatid \\
\hline 8 & degenerating spermatid? \\
\hline 9 & degenerating spermatid \\
\hline 10 & degenerating spermatid \\
\hline 11 & macrophage \\
\hline 12 & degenerating spermatid \\
\hline 13 & degenerating spermatid \\
\hline 14 & degenerating spermatid \\
\hline 15 & degenerating spermatid \\
\hline 16 & macrophage \\
\hline
\end{tabular}

\subsection{Analysing a sperm morphology smear}

\subsubsection{Assessment of normal sperm morphology}

It may be sufficient to determine the proportion of normal spermatozoa. With this morphology assessment paradigm, the functional regions of the spermatozoon are considered. It is unnecessary to distinguish all the variations in head size and shape or the various midpiece and principal piece defects.

Morphological evaluation should be performed on every assessable spermatozoon in several systematically selected areas of the slide, to prevent biased selection of particular spermatozoa.

- Examine the slide using brightfield optics at $\times 1000$ magnification with oil immersion.

- Assess all spermatozoa in each field, moving from one microscopic field to another.

- Evaluate at least 200 spermatozoa in each replicate, in order to achieve an acceptably low sampling error (see Box 2.5).

- Tally the number of normal and abnormal spermatozoa with the aid of a laboratory counter.

- Repeat the assessment of at least 200 spermatozoa, preferably on the replicate slide, but alternatively on the same slide. 
- Compare the percentages of normal morphological forms from the two independent assessments.

- Calculate the average and difference of the two percentages of normal forms from the replicate assessments.

- Determine the acceptability of the difference from Table 2.1 or Fig. A7.2, Appendix 7. (Each shows the maximum difference between two percentages that is expected to occur in $95 \%$ of samples because of sampling error alone.)

- If the difference between the percentages is acceptable, report the average percentage normal morphology. If the difference is too high, repeat the assessment on the same slides (see Box 2.6).

- Report the average percentage of normal forms to the nearest whole number.

Note 1: Assess only intact spermatozoa, defined as having a head and a tail (see Section 2.7.3), since only intact spermatozoa are counted for sperm concentration. Do not count immature germ (round) cells.

Note 2: Do not assess overlapping spermatozoa and those lying with the head on edge; these cannot be analysed adequately. They should not be present in a good smear (see Section 2.13.2.1), but may occur when debris and a large amount of particulate material are present (such as in viscous semen: see Section 2.13.2.3). These samples should be washed (see Section 2.13.2.4) and the slides examined before staining.

\subsubsection{Worked examples}

Example 1. The percentages of spermatozoa with normal morphology in replicate counts of 200 spermatozoa are 18 and 9 . The rounded average is $14 \%$ and the difference is $9 \%$. From Table 2.1 , it is seen that for an average of $14 \%$, a difference of up to $7 \%$ would be expected to occur by chance alone. As the observed difference exceeds this, the results are discarded and the slides reassessed in replicate.

Example 2. The percentages of spermatozoa with normal morphology in replicate counts of 200 spermatozoa are 10 and 14 . The rounded average is $12 \%$ and the difference is $4 \%$. From Table 2.1 , it is seen that for an average of $12 \%$, a difference of up to $7 \%$ would be expected to occur by chance alone. As the observed difference is less than this, the results are accepted and the mean value reported, namely $12 \%$ normal forms.

\subsubsection{Lower reference limit}

The lower reference limit for normal forms is $4 \%$ (5th centile, $95 \% \mathrm{CI} 3.0-4.0$ ).

Comment: The total number of morphologically normal spermatozoa in the ejaculate is of biological significance. This is obtained by multiplying the total number of spermatozoa in the ejaculate (see Section 2.8 .7 ) by the percentage of normal forms. 
2.17.4 Assessment of abnormal sperm morphology

Categorizing all abnormal forms of spermatozoa may be of diagnostic or research benefit. If desired, note the nature of the defects and calculate the percentage of spermatozoa with defects of the head $(\% \mathrm{H})$, midpiece $(\% \mathrm{M})$ or principal piece $(\% \mathrm{P})$, and those with excess residual cytoplasm (\% C).

A multi-key counter can be used, with one key for normal, one for abnormal, and one for each of the four abnormal categories $(H, M, P, C)$. Such a counter allows each spermatozoon to be counted only once, and each of its abnormalities to be scored separately.

- From the final assessment of 400 spermatozoa, it is possible to obtain the percentage of normal and abnormal spermatozoa (the two figures should add up to $100 \%$ ), as well as the percentage with each type of abnormality, i.e. $\% \mathrm{H}$, $\% \mathrm{M}, \% \mathrm{P}$ and $\% \mathrm{C}$ (these figures will not add up to $100 \%$ ).

- The percentage of spermatozoa in these abnormality classes is obtained by dividing the total number of abnormal spermatozoa with a specific defect by the total number of normal and abnormal spermatozoa scored $\times 100$. These numbers can also be used to calculate multiple anomalies indices (see Section 3.1).

\subsubsection{Worked example}

Example. Of 200 spermatozoa scored with a six-key counter for replicate 1, 42 spermatozoa are scored as normal and 158 as abnormal. Of the 158 abnormal spermatozoa, 140 have head defects, 102 have midpiece defects, 30 have principal piece defects, and 44 have excess residual cytoplasm. Results from replicate 2 are 36 normal and 164 abnormal spermatozoa, of which 122 have head defects, 108 midpiece defects, 22 principal piece defects, and 36 excess residual cytoplasm.

Only the normal category is compared for acceptability of replicates. Replicate 1 has $21 \%$ normal sperm and replic ate 2 has $18 \%$. The mean of these values is $19.5 \%$ (rounded up to $20 \%$ ), and the difference $3 \%$. From Table 2.1, it is seen that, for an average of $20 \%$, a difference of up to $8 \%$ would be expected to occur by chance alone. As the observed difference is less than this, the results are accepted and the mean values reported: normal forms $(42+36) / 400=20 \%$, abnormal heads $(140+122) / 400=66 \%$, abnormal midpieces $(102+108) / 400=53 \%$, abnormal principal pieces $(30+22) / 400=13 \%$, and percentage with excess residual cytyoplasm $(44+36) / 400=20 \%$.

Note: These categories do not add up to $100 \%$ since each abnormality is tallied separately and some spermatozoa have multiple defects.

Comment: A more detailed analysis of abnormal spermatozoa, with various indices combining the number of abnormalities in each region per abnormal spermatozoon, is given in Section 3.1.1. 


\subsubsection{Assessment of specific sperm defects}

Occasionally, many spermatozoa will have a specific structural defect. For example, the acrosome may fail to develop, giving rise to the "small round-head defect" or "globozoospermia". If the basal plate fails to attach to the nucleus at the opposite pole to the acrosome at spermiation, the heads are absorbed and only tails are found in semen (the pinhead defect).

Note 1: Pinheads (free tails) are not counted as head defects, since they possess no chromatin or head structure anterior to the basal plate.

Note 2: Because free tails (pinheads) and free heads are not counted as spermatozoa (defined as having a head and tail, see Section 2.7.3), they are not considered to be sperm abnormalities.

Men whose spermatozoa all display one of these defects are usually infertile. Such cases are rare, but it is critical that they are identified and correctly reported. Thus report the presence of specific sperm defects, e.g. free sperm heads, pinheads (free tails), heads lacking acrosomes.

If there are many such defects, their prevalence relative to spermatozoa can be determined. If $\mathrm{N}$ is the number of cells with defects counted in the same number of fields as 400 spermatozoa, and $S$ is the concentration of spermatozoa ( $10^{6}$ per $\mathrm{ml}$ ), then the concentration $(C)$ of the defects $\left(10^{6}\right.$ per $\left.\mathrm{ml}\right)$ can be calculated from the formula $\mathrm{C}=\mathrm{S} \times(\mathrm{N} / 400)$.

\subsection{Assessment of leukocytes in semen}

Leukocytes, predominantly polymorphonuclear leukocytes (PMN, neutrophils), are present in most human ejaculates (Tomlinson et al., 1993; J ohanisson et al., 2000). They can sometimes be differentiated from spermatids and spermatocytes in a semen smear stained with the Papanicolaou procedure (see Section 2.14.2). Differentiation is based on differences in staining coloration, and on nuclear size and shape (J ohanisson et al., 2000) (see Plates 6, 10, 11, 12, 13 and 14). Polymorphonuclear leukocytes can easily be confused morphologically with multinucleated spermatids, but stain a bluish colour, in contrast to the more pinkish colour of spermatids (J ohanisson et al., 2000). Nuclear size may also help identification: monocyte nuclei exhibit a wide variation in size, from approximately $7 \mu \mathrm{m}$ for lymphocytes to over $15 \mu \mathrm{m}$ for macrophages. These sizes are only guidelines, since degeneration and division affect the size of the nucleus.

There are several other techniques for quantifying the leukocyte population in semen. As peroxidase-positive granulocytes are the predominant form of leukocytes in semen, routine assay of peroxidase activity is useful as an initial screening technique (Wolff, 1995; J ohanisson et al., 2000) (see Section 2.18.1).

Leukocytes can be further differentiated with more time-consuming and expensive immunocytochemical assays against common leukocyte and sperm antigens (Homyk et al., 1990; Eggert-Kruse et al., 1992) (see Section 3.2). 
2.18.1 Staining cellular peroxidase using ortho-toluidine

This test is quick and inexpensive, and is a useful initial screening for granulocytes.

\subsubsection{Principle}

Traditionally, leukocytes in human semen are counted after a histochemical procedure that identifies the peroxidase enzyme, which is characteristic of granulocytes (Fig. 2.14). This technique has the advantage of being relatively easy to perform, but it does not detect:

- activated polymorphs which have released their granules;

- other types of leukocyte, such as lymphocytes, macrophages and monocytes, which do not contain peroxidase.

The test can be useful in distinguishing polymorphonuclear leukocytes from multinucleated spermatids, which are peroxidase-free (V ohanisson et al., 2000). The assay below is based on Nahoum \& Cardozo (1980). A kit for this is available commercially.

\subsubsection{Reagents}

1. Phosphate buffer, $67 \mathrm{mmol} / \mathrm{l}, \mathrm{pH} 6.0$ : dissolve $9.47 \mathrm{~g}$ of sodium hydrogen phosphate $\left(\mathrm{Na}_{2} \mathrm{HPO}_{4}\right)$ in $1000 \mathrm{ml}$ of purified water and $9.08 \mathrm{~g}$ of potassium dihydrogen phosphate $\left(\mathrm{KH}_{2} \mathrm{PO}_{4}\right)$ in $1000 \mathrm{ml}$ of purified water. Add one solution to the other (approximately $12 \mathrm{ml}$ of $\mathrm{Na}_{2} \mathrm{HPO}_{4}$ solution to $88 \mathrm{ml}$ of $\mathrm{KH}_{2} \mathrm{PO}_{4}$ solution) until the $\mathrm{pH}$ is 6.0 .

2. Saturated ammonium chloride $\left(\mathrm{NH}_{4} \mathrm{Cl}\right)$ solution: add $250 \mathrm{~g}$ of $\mathrm{NH}_{4} \mathrm{Cl}$ to $1000 \mathrm{ml}$ of purified water.

3. Disodium ethylenediamine tetra-acetic acid $\left(\mathrm{Na}_{2} \mathrm{EDTA}\right) 148 \mathrm{mmol} / \mathrm{l}$ : dissolve $50 \mathrm{~g} / \mathrm{l}$ in phosphate buffer $(\mathrm{pH} 6.0$ ) prepared in step 1.

4. Substrate: dissolve $2.5 \mathrm{mg}$ of o-toluidine in $10 \mathrm{ml}$ of $0.9 \%(9 \mathrm{~g} / \mathrm{l})$ saline.

5. Hydrogen peroxide $\left(\mathrm{H}_{2} \mathrm{O}_{2}\right) 30 \%(\mathrm{v} / \mathrm{v})$ : as purchased.

6. Working solution: to $9 \mathrm{ml}$ o-toluidine substrate, add $1 \mathrm{ml}$ of saturated $\mathrm{NH}_{4} \mathrm{Cl}$ solution, $1 \mathrm{ml}$ of $148 \mathrm{mmol} / / \mathrm{Na}_{2}$ EDTA, and $10 \mu \mathrm{l}$ of $30 \%(\mathrm{v} / \mathrm{v}) \mathrm{H}_{2} \mathrm{O}_{2}$ and mix well. This solution can be used up to 24 hours after preparation.

Note: The International Agency for Research on Cancer (IARC, 1982) has stated that ortho-toluidine should be regarded, for practical purposes, as if it presented a carcinogenic risk to humans. Take suitable precautions (see Appendix 2).

\subsubsection{Procedure}

1. Mix the semen sample well (see Box 2.3). 
2. Remove a $0.1-\mathrm{ml}$ aliquot of semen and mix with $0.9 \mathrm{ml}$ of working solution $(1+9$ (1:10) dilution).

3. Vortex the sperm suspension gently for 10 seconds and incubate at room temperature for 20-30 minutes. Alternatively, shake continuously with a tube-rocking system.

4. Remix the semen sample before removing a replicate aliquot and mixing with working solution as above.

2.18.1.4 Assessing peroxidase-positive cell number in the haemocytometer chambers

1. After 20-30 minutes, mix the sperm suspensions again and fill each side of a haemocytometer with one of the replicate preparations.

2. Store the haemocytometer horizontally for at least 4 minutes at room temperature in a humid chamber (e.g. on water-saturated filter paper in a covered Petri dish) to prevent drying out and to allow the cells to settle.

3. Examine the chamber with phase-contrast optics at $\times 200$ or $\times 400$ magnification.

4. Count at least 200 peroxidase-positive cells in each replic ate, in order to achieve an acceptably low sampling error (see Box 2.7 and Table 2.2). Peroxidase-positive cells are stained brown, while peroxidase-negative cells are unstained (see Fig. 2.14).

5. Examine one chamber, grid by grid, and continue counting until at least 200 peroxidase-positive cells have been observed and a complete grid has been examined. Counting must be done by complete grids; do not stop in the middle of a grid.

6. Make a note of the number of grids assessed to reach at least 200 peroxidasepositive cells. The same number of grids will be counted from the other chamber of the haemocytometer.

7. Tally the number of peroxidase-positive cells and grids with the aid of a laboratory counter.

8. Switch to the second chamber of the haemocytometer and perform the replicate count on the same number of grids as the first replicate, even if this yields fewer than 200 peroxidase-positive cells.

9. Calculate the sum and difference of the two numbers of peroxidase-positive cells.

10. Determine the acceptability of the difference from Table 2.5 or Fig. A7.1, Appendix 7. (Each shows the maximum difference between two counts that is expected to occur in $95 \%$ of samples because of sampling error alone.)

11. If the difference is acceptable, calculate the concentration (see Section 2.18.1.5). If the difference is too high, prepare two new dilutions and repeat the replicate count estimate (see Box 2.10). 
12. Report the average concentration of peroxidase-positive cells to two significant figures.

13. Calculate the total number of peroxidase-positive cells per ejaculate (see Comments after Section 2.18.1.8).

Fig. 2.14 Peroxidase-positive and -negative cells in human semen

A peroxidase-positive granulocyte (P) (brown colour) and a peroxidase-negative round cell (N). Scale bar $10 \mu \mathrm{m}$.

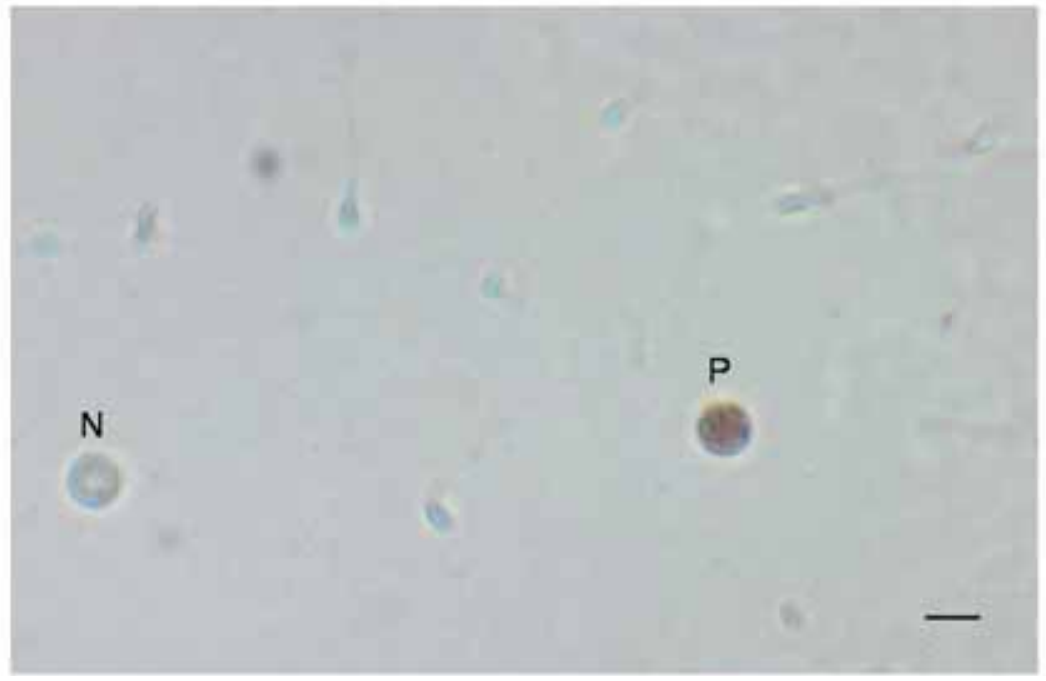

Micrograph courtesy of TG Cooper.

2.18.1.5 Calculation of the concentration of peroxidase-positive cells in semen

The concentration of peroxidase-positive cells in semen is their number ( $N$ ) divided by the volume of the total number $(n)$ of grids examined for the replicates (where the volume of a grid is $100 \mathrm{nl}$ ), multiplied by the dilution factor.

For a $1+9(1: 10)$ dilution, the concentration is $C=(N / n) \times(1 / 100) \times 10$ cells per $n l=$ $(\mathrm{N} / \mathrm{n}) \times(1 / 10)$ cells per $\mathrm{nl}$. Thus $(\mathrm{N} / \mathrm{n})$ is divided by 10 to obtain the concentration in peroxidase-positive cells per $\mathrm{nl}\left(10^{6}\right.$ cells per $\left.\mathrm{ml}\right)$.

When all nine grids in each chamber of the haemocytometer are assessed, the total number of peroxidase-positive cells can be divided by the total volume of both chambers $(1.8 \mu \mathrm{l})$, and multiplied by the dilution factor $(10)$, to obtain the concentration in cells per $\mu$ l (thousand cells per ml).

Note: This procedure can be used to calculate round cell concentration when the total number of round cells counted (peroxidase-positive and -negative) is used for $\mathrm{N}$ in the calculation. 


\subsubsection{Sensitivity of the method}

If there are fewer than 200 peroxidase-positive cells in the chamber, the sampling error will exceed $5 \%$. When fewer than 400 peroxidase-positive cells are found in all grids of both chambers, report the sampling error for the number of cells counted (see Table 2.2).

If fewer than 25 peroxidase-positive cells are counted in each chamber, the concentration will be $<277000$ cells per $\mathrm{ml}$; this is the lower limit of quantification for a sampling error of $20 \%$ when all nine grids of the improved Neubauer chamber are assessed and a $1+9$ (1:10) dilution is used (Cooper et al., 2006). Report the number of peroxidase-positive cells observed with the comment "Too few cells for accurate determination of concentration $(<277000 / \mathrm{ml})$ ".

Comment: The absence of peroxidase-positive cells from the aliquot examined does not necessarily mean that they are absent from the rest of the sample.

\subsubsection{Worked examples}

Example 1. With a $1+9(1: 10)$ dilution, replicate 1 is found to contain 60 peroxidase-positive cells in nine grids, while replicate 2 contains 90 peroxidase-positive cells in nine grids. The sum of the values $(60+90)$ is 150 in 18 grids and the difference $(90-60)$ is 30 . From Table 2.5 this is seen to exceed the difference expected by chance alone (24), so the results are discarded and new replicates are made.

Example 2. With a $1+9(1: 10)$ dilution, replicate 1 is found to contain 204 peroxidase-positive cells in five grids, while replicate 2 contains 198 peroxidase-positive cells in five grids. The sum of the values $(204+198)$ is 402 in 10 grids and the difference (204-198) is 6 . From Table 2.5 this is seen to be less than that found by chance alone (39), so the values are accepted.

The concentration of peroxidase-positive cells in the sample, for a $1+9$ (1:10) dilution, is $C=(N / n) \times(1 / 10)$ cells per $n l$ or $(402 / 10) / 10=4.02$ cells $/ \mathrm{nl}$, or $4.0 \times 10^{6}$ cells per $\mathrm{ml}$ (to two significant figures).

Example 3. With a $1+9(1: 10)$ dilution, replicate 1 is found to contain 144 peroxidase-positive cells in nine grids, while replicate 2 contains 162 peroxidase-positive cells in nine grids. The sum of the values $(144+162)$ is 306 in 18 grids and the difference (162-144) is 18 . From Table 2.5 this is seen to be less than that found by chance alone (34), so the values are accepted.

When all nine grids are assessed in each chamber, the concentration of the sample, for a $1+9(1: 10)$ dilution, is $C=(N / 1.8) \times 10$ cells per $\mu l=(306 / 1.8) \times 10=$ 1700 cells per $\mu$ or $1.7 \times 10^{6}$ cells per $\mathrm{ml}$ (to two significant figures). As fewer than 400 cells were counted, report the sampling error for 306 cells given in Table 2.2 (approximately 6\%).

Example 4. With a $1+9$ (1:10) dilution, no peroxidase-positive cells are found in either replicate. As fewer than 25 peroxidase-positive cells are found in all nine grids, the concentration is $<277000$ per ml; report that "No peroxidase-positive cells were seen in the samples. Too few cells for accurate determination of concentration (<277 000/ml)". 


\subsubsection{Reference value}

There is currently no reference range for peroxidase-positive cells in semen from fertile men. Pending additional evidence, this manual retains the consensus value of $1.0 \times 10^{6}$ peroxidase-positive cells per $\mathrm{ml}$ as a threshold value.

Comment 1: The total number of peroxidase-positive cells in the ejaculate may reflect the severity of an inflammatory condition (Wolff, 1995). This is obtained by multiplying the concentration of peroxidase-positive cells by the volume of the whole ejaculate.

Comment 2: Reports of cut-off values for peroxidase-positive cells in fertile men vary from $0.5 \times 10^{6}$ to $1.0 \times 10^{6} \mathrm{PMN}$ leukocytes per ml or from $1 \times 10^{6}$ to $2 \times 10^{6}$ total leukocytes per $\mathrm{ml}$ (Wolff, 1995). Previous editions of this manual have taken $1 \times 10^{6}$ leukocytes per $\mathrm{ml}$ as the threshold for leukocytospermia. Some have found this value too low (Wolff, 1995), while others consider it too high (Sharma et al., 2001; Punab et al., 2003), depending on the endpoint examined (semen quality, results of in-vitro fertilization, presence of bacteria, sperm response to reactive oxygen species).

Comment 3: Excessive numbers of leukocytes in the ejaculate (leukocytospermia, pyospermia) may be associated with infection and poor sperm quality.

Comment 4: Leukocyte-dependent damage to spermatozoa depends on the total leukocyte number in the ejaculate and the number of leukocytes relative to the number of spermatozoa.

Comment 5: Leukocytes can impair sperm motility and DNA integrity through an oxidative attack (see Section 4.1). However, whether the level of leukocytic infiltration observed is damaging depends on factors that are impossible to infer from a semen sample, such as the reason for, timing and anatomical location of the infiltration, as well as the nature of the leukocytes involved and whether they are in an activated state (Tomlinson et al., 1993; Aitken \& Baker, 1995; Rossi \& Aitken, 1997).

\subsection{Assessment of immature germ cells in semen}

Germ cells include round spermatids and spermatocytes, but rarely spermatogonia. They can be detected in stained semen smears, but may be difficult to distinguish from inflammatory cells when the cells are degenerating.

Spermatids and spermatocytes can usually be differentiated from leukocytes in a semen smear stained by the Papanicolaou procedure (J ohanisson et al., 2000) (see Section 2.14.2). Identification can be based on staining coloration, nuclear size and shape (see Plates 6, 10,11, 12, 13 and 14), absence of intracellular peroxidase (see Section 2.18), and lack of leukocyte-specific antigens (see Section 3.2). Multinucleated spermatids can easily be confused morphologically with polymorphonuclear leukocytes but stain a pinkish colour, in contrast to the more bluish PMN leukocytes (J ohanisson et al., 2000). Round spermatids may be identified with stains specific for the developing acrosome (Couture et al., 1976), lectins (see Section 4.4.1) or specific antibodies (Homyk et al., 1990; Ezeh et al., 1998). 
Nuclear size may also help in identification: spermatogonia (very rarely seen in semen) have a nucleus of approximately $8 \mu \mathrm{m}$, spermatocytes have a nucleus of approximately $10 \mu \mathrm{m}$, and spermatids have a nucleus of approximately $5 \mu \mathrm{m}$. These sizes are only guidelines, since degeneration and division affect the size of the nucleus.

\subsection{Testing for antibody coating of spermatozoa}

If spermatozoa demonstrate agglutination (i.e. motile spermatozoa stick to each other head-to-head, tail-to-tail or in a mixed way) (see Section 2.4.4), the presence of sperm antibodies may be the cause.

Comment 1: Sperm antibodies can be present without sperm agglutination; equally, agglutination can be caused by factors others than sperm antibodies.

Comment 2: The mere presence of sperm antibodies is insufficient for a diagnosis of sperm autoimmunity. It is necessary to demonstrate that the antibodies interfere severely with sperm function; this is usually done by a sperm-mucus penetration test (see Section 3.3). Antibodies can also interfere with zona binding and the acrosome reaction.

Anti-sperm antibodies (ASAs) in semen belong almost exclusively to two immunoglobulin classes: $\lg A$ and $\lg G$. IgM antibodies, because of their larger size, are rarely found in semen. $\lg A$ antibodies may have greater clinical importance than IgG antibodies (Kremer \& J ager, 1980). Both classes can be detected on sperm cells or in biological fluids in related screening tests.

- Tests for antibodies on spermatozoa ("direct tests"). Two direct tests are described here: the mixed antiglobulin reaction (MAR) test (for review see Bronson et al., 1984) and the immunobead (IB) test (Bronson et al., 1982; Clarke et al., 1982, 1985). The MAR test is performed on a fresh semen sample while the IB test uses washed spermatozoa. The results from the two tests do not always agree (MacM illan \& Baker, 1987; Scarselli et al., 1987; Meinertz \& Bronson, 1988; Hellstrom et al., 1989), but IB test results are well correlated with the results of the immobilization test that detects antibodies in serum. The experimental protocols for the IB and MAR tests vary, but for both the sperm/ bead preparation is examined with a microscope. The beads adhere to the motile and immotile spermatozoa that have surface-bound antibodies; the percentage of motile spermatozoa with bound beads is recorded.

- Tests for anti-sperm antibodies in sperm-free fluids, i.e. seminal plasma, blood serum and solubilized cervical mucus ("indirect" tests). In these tests, the diluted, heat-inactivated fluid suspected of containing ASAs is incubated with antibody-free donor spermatozoa that have been washed free of seminal fluid. Any ASAs in the suspect fluid will bind specifically to the donor spermatozoa, which are then assessed in a direct test, as above. For reliable results, it is important to allow sufficient time for the sperm-antibody interaction, since it may take up to 10 minutes for the mixed agglutination to become visible. However, it should be borne in mind that sperm motility declines with time, and the tests depend on the presence of motile spermatozoa. 
Note 1: The two ASA tests described here are commercially available. Both depend on the presence of motile spermatozoa. If there are insufficient motile spermatozoa, indirect tests on seminal plasma or blood serum can be used.

Note 2: Cytotoxic antibodies that kill all spermatozoa or inhibit sperm motility cannot be detected with these assays.

2.20.1 The mixed antiglobulin reaction test

The mixed antiglobulin reaction (MAR) test is an inexpensive, quick and sensitive screening test (Rajah et al., 1992), but it provides less information than the direct immunobead test (see Section 2.20.2).

In the MAR test, a "bridging" antibody (anti- $\lg G$ or anti- $\lg A$ ) is used to bring the antibody-coated beads into contact with unwashed spermatozoa in semen bearing surface $\lg G$ or $\operatorname{Ig} A$. The direct IgG and IgA MAR tests are performed by mixing fresh, untreated semen separately with latex particles (beads) or treated red blood cells coated with human IgG or IgA. To the suspensions is added a monospecific anti-human-IgG or anti-human- IgA. The formation of mixed agglutinates between particles and motile spermatozoa indicates the presence of IgG or IgA antibodies on the spermatozoa. (Agglutination between beads serves as a positive control for antibody-antigen recognition.)

\subsubsection{Procedure}

1. Mix the semen sample well (see Box 2.3).

2. Remove replicate aliquots of $3.5 \mu \mathrm{l}$ of semen and place on separate microscope slides.

3. Include one slide with $3.5 \mu$ of ASA-positive semen and one with $3.5 \mu$ of ASAnegative semen as controls in each direct test. This semen should be from men with and without anti-sperm antibodies, respectively, as shown in previous direct MAR tests. Alternatively, positive spermatozoa can be produced by incubation in serum known to contain antibodies (see Section 2.20.3).

4. Add $3.5 \mu \mathrm{l}$ of IgG-coated latex particles (beads) to each droplet of test and control semen, and mix by stirring with the pipette tip.

5. Add $3.5 \mu$ l of antiserum against human IgG to each semen-bead mixture, and mix by stirring with the pipette tip.

6. Cover the suspension with a coverslip $(22 \mathrm{~mm} \times 22 \mathrm{~mm})$ to provide a depth of approximately $20 \mu \mathrm{m}$ (see Box 2.4).

7. Store the slide horizontally for 3 minutes at room temperature in a humid chamber (e.g. on water-saturated filter paper in a covered Petri dish) to prevent drying out.

8. Examine the wet preparation with phase-contrast optics at $\times 200$ or $\times 400$ magnification after 3 minutes and again after 10 minutes. 
9. Repeat the procedure using $\lg A$ - instead of $\lg G$-coated beads and anti- $\lg A$ instead of anti-IgG antibodies.

\subsubsection{Scoring}

If spermatozoa have antibodies on their surface, the latex beads will adhere to them. The motile spermatozoa will initially be seen moving around with a few or even a group of particles attached. Eventually the agglutinates become so massive that the movement of the spermatozoa is severely restricted. Sperm that do not have coating antibodies will be seen swimming freely between the particles.

The goal of the assay is to determine the percentage of motile spermatozoa that have beads attached to them. A common problem occurs with NP spermatozoa that are close to beads, but are not attached. Whether the beads are bound can often be verified by lightly tapping the coverslip with a small pipette tip: the movement of beads in concert with active spermatozoa is indicative of positive binding.

1. Score only motile spermatozoa and determine the percentage of motile spermatozoa that have two or more latex particles attached. Ignore tail-tip binding.

2. Evaluate at least 200 motile spermatozoa in each replicate, in order to achieve an acceptably low sampling error (see Box 2.5).

3. Calculate the percentage of motile spermatozoa that has particles attached.

4. Record the class ( $\lg G$ or $\lg A)$ and the site of binding of the latex particles to the spermatozoa (head, midpiece, principal piece). Ignore tail-tip binding.

Note 1: If $100 \%$ of motile spermatozoa are bead-bound at 3 minutes, take this as the test result; do not read again at 10 minutes.

Note 2: If less than $100 \%$ of motile spermatozoa are bead-bound at 3 minutes, read the slide again at 10 minutes.

Note 3: If spermatozoa are immotile at 10 minutes, take the value at 3 minutes as the result.

\subsubsection{Reference value}

There are currently no reference values for antibody-bound spermatozoa in the MAR test of semen from fertile men. Pending additional evidence, this manual retains the consensus value of $50 \%$ motile spermatozoa with adherent particles as a threshold value.

Comment: Sperm penetration into the cervical mucus and in-vivo fertilization tend to be significantly impaired when $50 \%$ or more of the motile spermatozoa have antibody bound to them (Abshagen et al., 1998). Particle binding restricted to the tail tip is not associated with impaired fertility and can be present in fertile men (Chiu \& Chamley, 2004). 
2.20.2 The direct immunobead test

This assay is more time-consuming than the MAR test but provides information about antibodies on spermatozoa that have been removed from possible masking components in seminal plasma.

In the direct immunobead (IB) test, beads coated with covalently-bound rabbit anti-human immunoglobulins against IgG or IgA are mixed directly with washed spermatozoa. The binding of beads with anti-human IgG or IgA to motile spermatozoa indicates the presence of $\lg G$ or $\lg A$ antibodies on the surface of the spermatozoa.

\subsubsection{Reagents}

1. Dulbecco's glucose-phosphate-buffered saline (PBS)-bovine serum albumin (BSA) or Tyrode's-BSA solution: see Appendix 4, sections A4.2 and A4.9.

2. Buffer I: add $0.3 \mathrm{~g}$ of Cohn Fraction V BSA to $100 \mathrm{ml}$ of Dulbecco's PBS or Tyrode's medium.

3. Buffer II: add $5 \mathrm{~g}$ of Cohn Fraction V BSA to $100 \mathrm{ml}$ of Dulbecco's PBS or Tyrode's medium.

4. Filter all solutions through $0.45-\mu \mathrm{m}$ filters and warm to $25-35^{\circ} \mathrm{C}$ before use.

\subsubsection{Preparing the immunobeads}

1. For each immunobead type $(\lg G, \lg A)$, add $0.2 \mathrm{ml}$ of stock bead suspension to $10 \mathrm{ml}$ of buffer I in separate centrifuge tubes.

2. Centrifuge at $500 \mathrm{~g}$ or $600 \mathrm{~g}$ for 5-10 minutes.

3. Decant and discard the supernatant from the washed immunobeads.

4. Gently resuspend the beads in $0.2 \mathrm{ml}$ of buffer II.

\subsubsection{Preparing the spermatozoa}

The amount of semen required for these assays is determined from the sperm concentration and motility, as shown in Table 2.7.

Table 2.7 How much semen to use for an immunobead test

\begin{tabular}{|c|c|c|}
\hline $\begin{array}{c}\text { Sperm concentration } \\
\left(10^{6} / \mathrm{ml}\right)\end{array}$ & $\begin{array}{c}\text { Sperm motility } \\
(\mathrm{PR})(\%)\end{array}$ & $\begin{array}{c}\text { Volume of semen required } \\
(\mathrm{ml})\end{array}$ \\
\hline$>50$ & - & 0.2 \\
\hline $21-50$ & $>40$ & 0.4 \\
\hline $21-50$ & $<40>10$ & 0.8 \\
\hline $10-20$ & $>40$ & 1.0 \\
\hline $10-20$ & $<40>10$ & 2.0 \\
\hline$<10>5$ & $>10$ & $>2.0$ \\
\hline
\end{tabular}


1. Mix the semen sample well (see Box 2.3).

2. Transfer the required amount of semen to a centrifuge tube and make up to $10 \mathrm{ml}$ with buffer $\mathrm{I}$.

3. Centrifuge at $500 \mathrm{~g}$ for $5-10$ minutes.

4. Decant and discard the supernatant from the washed spermatozoa.

5. Gently resuspend the sperm pellet in $10 \mathrm{ml}$ of fresh buffer I.

6. Centrifuge again at $500 \mathrm{~g}$ for 5-10 minutes.

7. Decant and discard the supernatant.

8. Gently resuspend the sperm pellet in $0.2 \mathrm{ml}$ of buffer II.

Note 1: Aliquots of more than $1.0 \mathrm{ml}$ require three washings.

Note 2: Samples with low sperm motility (e.g. 10\% or less) may not provide clearcut results. In this case, consider the indirect immunobead test (see Section 2.20.3).

\subsubsection{Procedure}

ASA-positive spermatozoa and ASA-negative spermatozoa should be included as controls in each test. Semen should be from men with and without anti-sperm antibodies, respectively, as detected in previous direct immunobead tests.

1. Place $5 \mu \mathrm{l}$ of the washed sperm suspension being tested on a microscope slide.

2. Prepare separate slides with $5 \mu$ l of ASA-positive spermatozoa and $5 \mu$ l of ASAnegative spermatozoa.

3. Add $5 \mu$ l of anti-lgG immunobead suspension beside each sperm droplet.

4. Mix each anti-IgG immunobead and sperm droplet together by stirring with the pipette tip.

5. Place a $22 \mathrm{~mm} \times 22 \mathrm{~mm}$ coverslip over the mixed droplet to provide a depth of approximately $20 \mu \mathrm{m}$ (see Box 2.4).

6. Store the slides horizontally for 3-10 minutes at room temperature in a humid chamber (e.g. on water-saturated filter paper in a covered Petri dish). Do not wait longer than 10 minutes before assessing the slides, since immunobead binding decreases significantly during incubation (Gould et al., 1994).

7. Examine the slides with phase-contrast optics at $\times 200$ or $\times 400$ magnification.

8. Score only motile spermatozoa that have one or more beads bound, as described in Section 2.20.1.2. Ignore tail-tip binding.

9. Interpret the test as described in Section 2.20.1.3.

10. Repeat the procedure using the anti- IgA immunobead suspension. 
Note: In order to ensure that all binding is assessed within 10 minutes, it is best to stagger the preparation of the slides.

\subsubsection{Reference value}

There are currently no reference values for antibody-bound spermatozoa in the IB test of semen from fertile men. Pending additional evidence, this manual retains the consensus value of $50 \%$ motile spermatozoa with adherent particles as a threshold value.

Comment: The diagnosis of immunological infertility is made when $50 \%$ or more of the motile spermatozoa (progressive and non-progressive) have adherent particles (B arratt et al., 1992). Particle binding restricted to the tail tip is not associated with impaired fertility and can be present in fertile men (Chiu \& Chamley, 2004).

\subsubsection{The indirect immunobead test}

The indirect immunobead test is used to detect anti-sperm antibodies in heatinactivated, sperm-free fluids (serum, testicular fluid, seminal plasma or bromelain-solubilized cervical mucus). Antibody-free donor's spermatozoa take up anti-sperm antibodies present in the tested fluid and are then assessed as in the direct immunobead test.

\subsubsection{Reagents}

See Section 2.20.2.1 (reagents for the direct IB test).

If cervical mucus is to be tested, prepare $10 \mathrm{IU} / \mathrm{ml}$ bromelain, a broad-specificity proteolytic enzyme (EC 3.4.22.32) (see Box 2.2).

\subsubsection{Preparing the immunobeads}

See Section 2.20.2.2.

\subsubsection{Preparing the donor's spermatozoa}

See Section 2.20.2.3.

\subsubsection{Preparing the fluid to be tested}

1. If testing cervical mucus, dilute $1+1(1: 2)$ with $10 \mathrm{lU} / \mathrm{ml}$ bromelain, stir with a pipette tip and incubate at $37{ }^{\circ} \mathrm{C}$ for 10 minutes. When liquefaction is complete, centrifuge at $2000 \mathrm{~g}$ for 10 minutes. Use the supernatant immediately for testing, or freeze at $-70^{\circ} \mathrm{C}$.

2. Inactivate any complement in the solublized cervical mucus, serum, seminal plasma or testicular fluid by heating at $56{ }^{\circ} \mathrm{C}$ for $30-45$ minutes.

3. Dilute the heat-inactivated sample $1+4(1: 5)$ with buffer II (e.g. $10 \mu$ l of the body fluid to be tested with $40 \mu$ l of buffer II). 
4. Include known-positive and -negative samples, e.g. serum from men with and without anti-sperm antibodies, respectively, as detected in the indirect immunobead test, as controls in each indirect test. Men who have had a vasectomy can be a source of serum if positive ( $>50 \%$ motile spermatozoa with bead binding, excluding tail-tip binding).

2.20.3.5 Incubating the donor's spermatozoa with the fluid to be tested

1. Mix $50 \mu$ l of washed donor sperm suspension with $50 \mu$ of $1+4(1: 5)$ diluted fluid to be tested.

2. Incubate at $37^{\circ} \mathrm{C}$ for 1 hour.

3. Centrifuge at $500 \mathrm{~g}$ for 5-10 minutes.

4. Decant and discard the supernatant.

5. Gently resuspend the sperm pellet in $10 \mathrm{ml}$ of fresh buffer $\mathrm{l}$.

6. Centrifuge again at $500 \mathrm{~g}$ for $5-10$ minutes.

7. Decant and discard the supernatant.

8. Repeat the washing steps 5,6 and 7 above.

9. Gently resuspend the sperm pellet in $0.2 \mathrm{ml}$ of buffer II.

\subsubsection{Immunobead test}

1. Perform the IB test, as described in Section 2.20.2.4, with the fluid-incubated donor spermatozoa.

2. Score and interpret the test as described in Sections 2.20.1.2 and 2.20.1.3. 


\section{CHAPTER 3 Optional procedures}

The tests described in this chapter are not necessary for routine semen analysis, but may be useful in certain circumstances for diagnostic or research purposes.

\subsection{Indices of multiple sperm defects}

Morphologically abnormal spermatozoa often have multiple defects (of the head, midpiece or principal piece, or combinations of these defects). A detailed assessment of the incidence of morphological abnormalities may be more useful than a simple evaluation of the percentage of morphologically normal spermatozoa, especially in studies of the extent of damage to human spermatogenesis (J ouannet et al., 1988; Auger et al., 2001). Recording the morphologically normal spermatozoa, as well as those with abnormalities of the head, midpiece and principal piece, in a multiple-entry system gives the mean number of abnormalities per spermatozoon assessed.

Three indices can be derived from records of the detailed abnormalities of the head, midpiece and principal piece in a multiple-entry system:

- the multiple anomalies index (MAI) (J ouannet et al., 1988);

- the teratozoospermia index (TZI) (Menkveld \& Kruger, 1996; Menkveld et al., 2001);

- the sperm deformity index (SDI) (Aziz et al., 1996, 2004).

These indices have been correlated with fertility in vivo (MAI and TZI) ( ouannet et al., 1988; Menkveld et al., 2001; Slama et al., 2002) and in vitro (SDI) (Aziz et al., 1996), and may be useful in assessments of certain exposures or pathological conditions (Auger et al., 2001; Aziz et al., 2004).

\subsubsection{Calculation of indices of multiple morphological defects}

Each abnormal spermatozoon is scored for defects of the head, midpiece and principal piece, and for the presence of excess residual cytoplasm (volume more than one third of the sperm head size). Laboratory cell counters can be used, with the number of entry keys adapted to the type of index being assessed. If a counter is not available, a simple score sheet can be used.

- The MAl is the mean number of anomalies per abnormal spermatozoon. All the head, midpiece and principal piece anomalies are included in the calculation. The morphology criteria used for this analysis are from David et al. (1975), as modified by Auger \& Eustache (2000), and differ from those presented in this manual (Sections 2.15.1 and 2.15.2).

- The TZI is similar to the MAI, but a maximum of four defects per abnormal spermatozoon is counted: one each for head, midpiece, and principal piece and one for excess residual cytoplasm, whatever the real number of anomalies per abnormal spermatozoon. The morphological criteria given in this manual can be used. 
- The SDI is the number of defects divided by the total number of spermatozoa (not only the abnormal spermatozoa). It incorporates several categories of head anomaly but only one for each midpiece and principal piece defect. The morphological criteria given in this manual can be used.

Table 3.1 Calculation of indices of multiple sperm defects

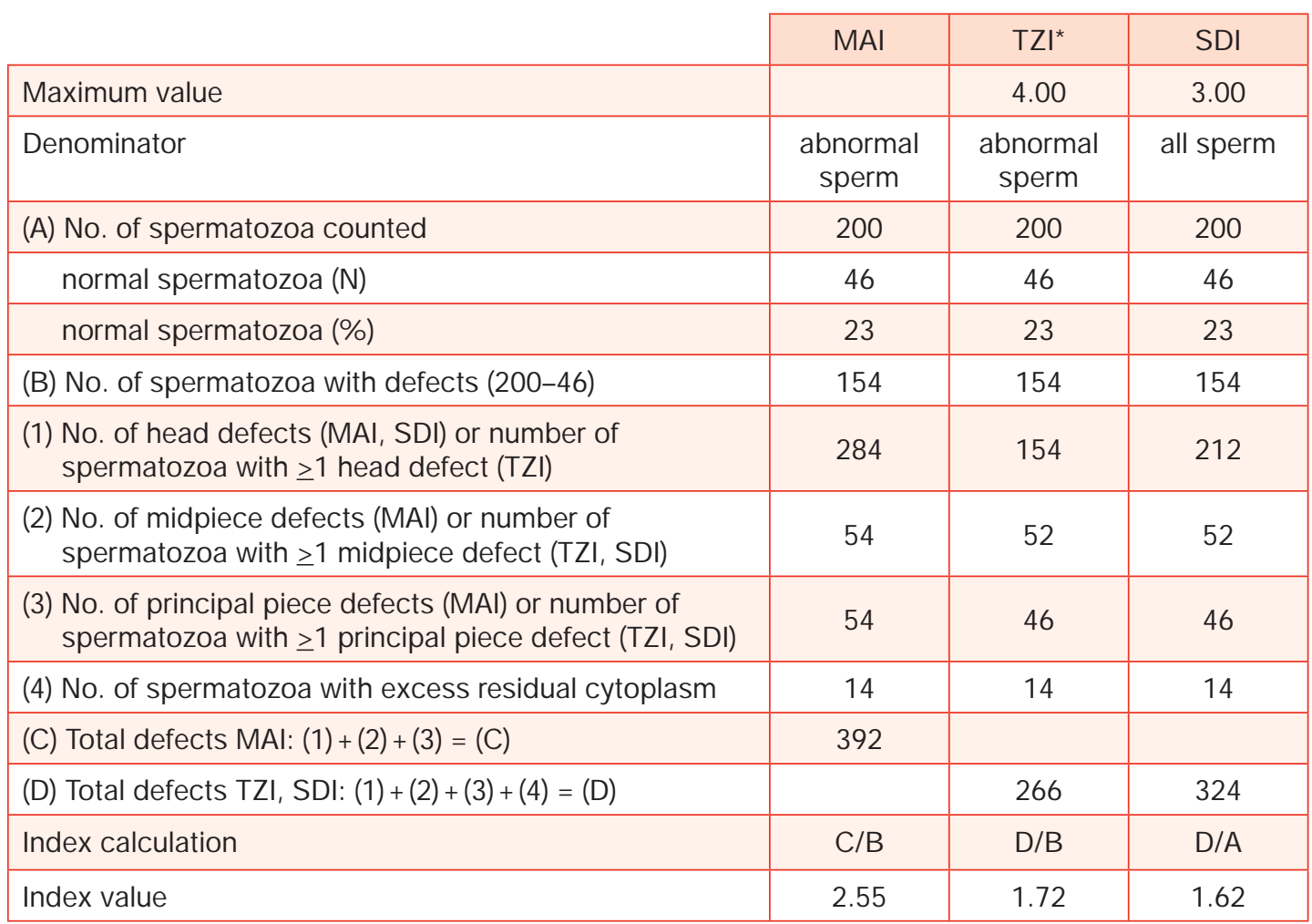

*This description of the TZI is in accordance with that in the original paper (Menkveld et al., 2001) and the manual of the European Society of Human Reproduction and Embryology (ESHRE) and the Nordic Association for Andrology (NAFA) (ESHRE/NAFA, 2002), which give values ranging from 1 to 4 . This is different from the description in the previous edition of this manual (WHO, 1999), in which excess residual cytoplasm was considered a midpiece defect, and which gave TZI values ranging from 1 to 3 .

\subsubsection{Worked example}

Example. Of 200 spermatozoa scored with a six-key counter for replicate 1, 42 were scored as normal and 158 as abnormal. Of the 158 abnormal spermatozoa, 140 had head defects, 102 midpiece defects, 30 principal piece defects, and 44 excess residual cytoplasm. Results from replicate 2 were: 36 normal and 164 abnormal, of which 122 had head defects, 108 midpiece defects, 22 principal piece defects, and 36 excess residual cytoplasm. To determine the TZI, divide the total number of defects determined $(140+102+30+44+122+108+22+36=604$ abnormalities) by the number of abnormal spermatozoa $(158+164=322)$, i.e. $\mathrm{TZI}=604 / 322=1.88$. 
Table 3.2 presents values for MAI and TZI for men attending infertility clinics and men who had fathered a child within the last 3 years.

Table 3.2 Sperm defect indices for men from fertile and infertile couples

\begin{tabular}{|c|c|c|c|c|}
\hline & \multicolumn{2}{|c|}{ Infertile couples } & \multicolumn{2}{|c|}{ Fertile couples } \\
\hline & $\mathrm{MAl}^{1}$ & $\mathrm{TZI}^{2}$ & $\mathrm{MAl}^{3}$ & $\mathrm{TZI}^{2}$ \\
\hline Mean & 1.94 & 1.81 & 1.58 & 1.51 \\
\hline SD & 0.37 & 0.3 & 0.2 & 0.2 \\
\hline Minimum & 1.12 & 1.26 & 1.04 & 1.17 \\
\hline Maximum & 3.9 & 2.64 & 2.38 & 2.07 \\
\hline \multicolumn{5}{|l|}{ Centiles } \\
\hline 5 & 1.44 & & 1.27 & \\
\hline 10 & 1.51 & 1.74 & 1.34 & 1.33 \\
\hline 25 & 1.67 & & 1.44 & \\
\hline 50 & 1.88 & 1.81 & 1.58 & 1.54 \\
\hline 75 & 2.14 & & 1.72 & \\
\hline 90 & 2.44 & & 1.86 & \\
\hline 95 & 2.65 & & 1.94 & \\
\hline $\mathrm{N}$ & 4930 & 103 & 994 & 107 \\
\hline
\end{tabular}

${ }^{1}$ Unpublished data from J Auger, Paris, using David morphological classification (David et al., 1975, modified by Auger \& Eustache, 2000).

2 Menkveld et al., 2001.

${ }^{3}$ J ørgensen et al., 2001, using David morphological classification (David et al., 1975; modified by Auger \& Eustache, 2000).

\subsection{Panleukocyte (CD45) immunocytochemical staining}

Polymorphonuclear leukocytes that have released their granules, and other species of leukocyte, such as lymphocytes, macrophages or monocytes, which do not contain peroxidase, cannot be detected by the o-toluidine test for cellular peroxidase (see Section 2.18.1), but can be detected by immunocytochemical means. Immunocytochemical staining is more expensive and time-consuming than assessing granulocyte peroxidase activity, but is useful for distinguishing between leukocytes and germ cells.

\subsubsection{Principle}

All classes of human leukocytes express a specific antigen (CD45) that can be detected with an appropriate monoclonal antibody. By changing the nature of the primary antibody, this general procedure can be adapted to allow detection of different types of leukocyte, such as macrophages, monocytes, neutrophils, B-cells or T-cells, should they be the focus of interest. 


\subsubsection{Reagents}

1. Dulbecco's phosphate-buffered saline (DPBS): see Appendix 4, section A4.2.

2. Tris-buffered saline (TBS), $\mathrm{pH}$ 8.2; see Appendix 4, section A4.8.

3. Tetramisole- $\mathrm{HCl}$ (levamisole) $1.0 \mathrm{~mol} / \mathrm{l}$ : dissolve $2.4 \mathrm{~g}$ levamisole in $10 \mathrm{ml}$ of purified water.

4. Substrate: to $9.7 \mathrm{ml}$ of TBS (pH 8.2) add $2 \mathrm{mg}$ of naphthol AS-MX phosphate, $0.2 \mathrm{ml}$ of dimethylformamide and $0.1 \mathrm{ml}$ of $1.0 \mathrm{~mol} / /$ levamisole. J ust before use, add $10 \mathrm{mg}$ of Fast Red TR salt and filter $(0.45-\mu \mathrm{m}$ pore size).

5. Fixative: acetone alone or acetone/methanol/formaldehyde: to $95 \mathrm{ml}$ of acetone add $95 \mathrm{ml}$ of absolute methanol and $10 \mathrm{ml}$ of $37 \%(\mathrm{v} / \mathrm{v})$ formaldehyde.

6. Primary antibody: a mouse monoclonal antibody against the common leukocyte antigen, encoded CD45.

7. Secondary antibody: anti-mouse rabbit immunoglobulins. The dilution used will depend on the antibody titre and source.

8. Alkaline phosphatase-anti-alkaline phosphatase complex (APAAP).

9. Harris's haematoxylin staining mixture (as counterstain): see Appendix 4, section A4.10.

\subsubsection{Procedure}

3.2.3.1 Preparing the semen

1. Mix the semen sample well (see Box 2.3).

2. Mix an aliquot of approximately $0.5 \mathrm{ml}$ with five volumes of DPBS.

3. Centrifuge at $500 \mathrm{~g}$ for 5 minutes, remove the supernatant and suspend the sperm pellet in five times its volume of DPBS.

4. Centrifuge at $500 \mathrm{~g}$ for 5 minutes.

5. Repeat this procedure once more and resuspend the pellet in DPBS to approximately $50 \times 10^{6}$ spermatozoa per $\mathrm{ml}$.

\subsubsection{Preparing the sperm smears}

1. Make replicate smears on clean glass slides (see Section 2.13.2) from 5- $\mu$ l aliquots of the suspension and allow them to air-dry.

2. Fix the air-dried cells in absolute acetone for 10 minutes or in acetone/ethanol/ formaldehyde for 90 seconds.

3. Wash twice with TBS and allow the slides to drain.

4. The slides can then be stained immediately or wrapped in aluminium foil and stored at $-70{ }^{\circ} \mathrm{C}$ for later analysis. 


\subsubsection{Incubating with antibodies}

1. On each slide, mark an area of fixed cells (a circle of about $1 \mathrm{~cm}$ diameter) with a grease pencil (delimiting pen) and cover the area with $10 \mu \mathrm{l}$ of primary monoclonal antibody.

2. Store the slide horizontally for 30 minutes at room temperature in a humid chamber (e.g. on water-saturated filter paper in a covered Petri dish) to prevent drying out.

3. Wash the slides twice with TBS and allow them to drain.

4. Cover the same area of the smear with $10 \mu \mathrm{l}$ of secondary antibody and incubate for 30 minutes in a humid chamber at room temperature.

5. Wash twice with TBS and allow the slides to drain.

6. Add $10 \mu$ l of APAAP to the same area.

7. Incubate for 1 hour in a humid chamber at room temperature.

8. Wash twice in TBS and allow the slides to drain.

9. Incubate with $10 \mu \mathrm{l}$ of naphthol phosphate substrate for 20 minutes in a humid chamber at room temperature.

Note: In order to intensify the reaction product, staining with the secondary antibody and APAAP can be repeated, with a 15-minute incubation period for each reagent.

\subsubsection{Counterstaining and mounting}

1. Once the slides have developed a reddish colour, wash with TBS.

2. Counterstain for a few seconds with haematoxylin; wash in tap water and mount in an aqueous mounting medium (see Sections 2.14.2.4 and 2.14.2.5).

\subsubsection{Assessing CD45-positive cell numbers}

1. Examine the entire stained area of the slide with brightfield optics at $\times 200$ or x400 magnification. CD45-positive cells (leukocytes) are stained red (see Fig. 3.1).

2. Score separately CD45-positive cells and spermatozoa until at least 200 spermatozoa have been observed in each replicate, in order to achieve an acceptably low sampling error (see Box 2.7 and Table 2.2).

3. Tally the number of CD45-positive cells and spermatozoa with the aid of a laboratory counter.

4. Assess the second smear in the same way (until 200 spermatozoa have been counted). 
5. Calculate the sum and difference of the two counts of CD45-positive cells.

6. Determine the acceptability of the difference from Table 2.5 or Fig. A7.1; Appendix 7. (Each shows the maximum difference between two counts that is expected to occur in $95 \%$ of samples because of sampling error alone.)

7. If the difference is acceptable, calculate the concentration (see Section 3.2.3.6). If the difference is too high, reassess the slides in replicate (see Box 2.10).

8. Report the average concentration of CD45-positive cells to two significant figures.

9. Calculate the total number of CD45-positive cells per ejaculate (see Comment after Section 3.2.3.9).

\subsubsection{Calculation of the concentration of CD45-positive cells in semen}

The concentration of CD45-positive cells is calculated relative to that of spermatozoa on the slide. If $\mathrm{N}$ is the number of CD45-positive cells counted in the same number of fields as 400 spermatozoa, and $S$ is the concentration of spermatozoa $\left(10^{6}\right.$ per $\left.\mathrm{ml}\right)$, then the concentration $(\mathrm{C})$ of $\mathrm{CD} 45$-positive cells $\left(10^{6}\right.$ per $\left.\mathrm{ml}\right)$ can be calculated from the formula $\mathrm{C}=\mathrm{S} \times(\mathrm{N} / 400)$.

\section{Fig. 3.1 Leukocytes in semen}

CD45-bearing cells (leukocytes) are stained red.

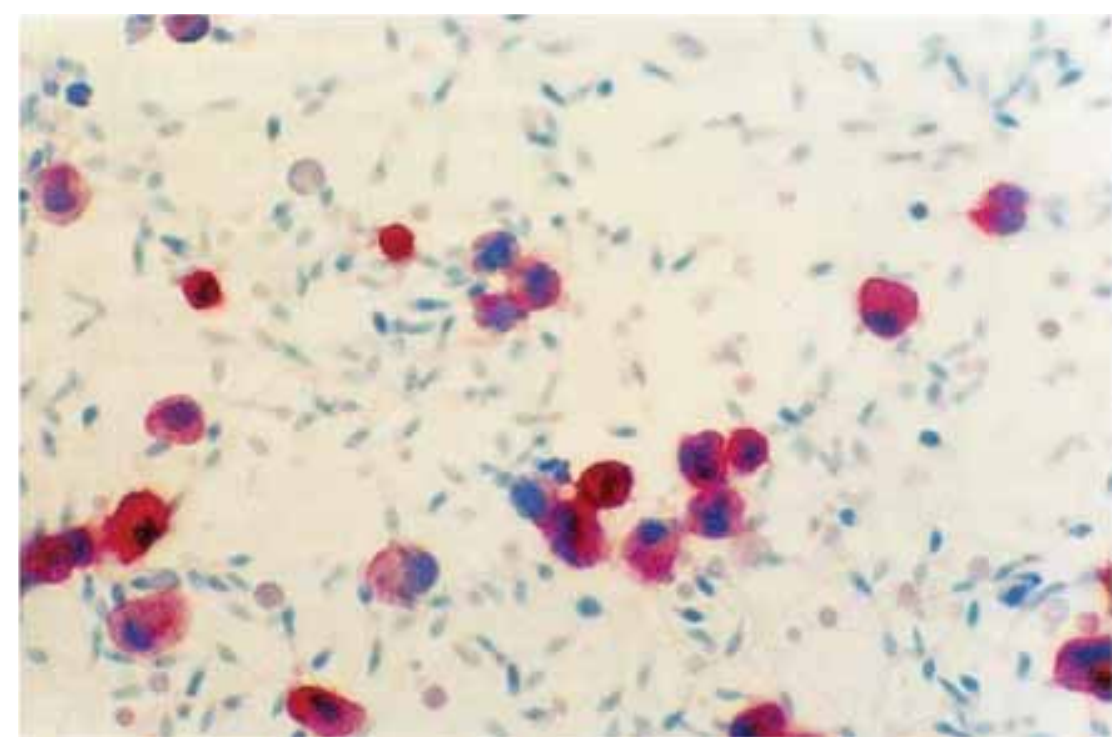

Micrograph courtesy of RJ Aitken. 


\subsubsection{Sensitivity of the method}

If there are fewer CD45-positive cells than spermatozoa in the sample (i.e. $<400$ ), the sampling error will exceed $5 \%$. In this case, report the sampling error for the number of cells counted (see Table 2.2).

If fewer than 25 CD45-positive cells are counted, report the number of CD45positive cells observed with the comment "Too few for accurate determination of concentration".

\subsubsection{Worked examples}

Example 1. In replicate 1 there are 20 CD45-positive cells per 200 spermatozoa, while in replicate 2 there are 40 CD 45 -positive cells per 200 spermatozoa. The sum of the values $(20+40)$ is 60 and the difference $(40-20)$ is 20 . From Table 2.5 this is seen to exceed the difference expected by chance alone (15), so the results are discarded and new assessments are made.

Example 2. In replicate 1 there are 25 CD 45-positive cells per 200 spermatozoa and in replicate 2 there are 35 CD 45-positive cells per 200 spermatozoa. The sum of the values $(25+35)$ is 60 and the difference $(35-25)$ is 10 . From Table 2.5 this is seen to be less than that found by chance alone (15), so the values are accepted.

For 60 CD45-positive cells per 400 spermatozoa and a sperm concentration of $70 \times 10^{6}$ cells per $\mathrm{ml}$, the $\mathrm{CD} 45$ - positive cell concentration is $\mathrm{C}=\mathrm{S} \times(\mathrm{N} / 400)$ cells per $\mathrm{ml}=70 \times 10^{6} \times(60 / 400)=10.5 \times 10^{6}$ cells per $\mathrm{ml}$, or $10 \times 10^{6}$ cells per $\mathrm{ml}$ (to two significant figures). As fewer than 400 cells were counted, report the sampling error for 60 cells given in Table 2.2 (approximately 13\%).

\subsubsection{Reference value}

There are currently no reference values for CD45-positive cells in semen from fertile men. The consensus threshold value of $1.0 \times 10^{6}$ cells per $\mathrm{ml}$ for peroxidasepositive cells (see Section 2.18.1.8) implies a higher concentration of total leukocytes, since not all leukocytes are peroxidase-positive granulocytes.

Comment: The total leukocyte number (total number of leukocytes in the ejaculate) may reflect the severity of an inflammatory condition (Wolff, 1995). The total number of CD45-positive cells in the ejaculate is obtained by multiplying the CD45-positive cell concentration by the total volume of the ejaculate. 


\subsection{Interaction between spermatozoa and cervical mucus}

Cervical mucus is receptive to spermatozoa for a limited time during the menstrual cycle (at mid-cycle), when the estrogen-influenced mucus favours sperm penetration. The length of time during which spermatozoa can penetrate cervical mucus varies considerably among women, and may vary in the same individual from one cycle to another.

Note: See Appendix 5 for details of collection, storage and evaluation of the characteristics of cervical mucus.

Comment: When a man cannot provide a semen sample, the postcoital test (see Section 3.3.1) can provide some information about his spermatozoa.

\subsubsection{In-vivo (postcoital) test}

\subsubsection{Purpose}

The aims of a postcoital test are to determine the number of active spermatozoa in the cervical mucus and to evaluate sperm survival (Sobrero \& MacLeod, 1962) and sperm behaviour some hours after coitus (the reservo ir role of mucus) (Moghissi, 1976). This information may be used to assess the significance of a positive sperm antibody test in the male or female partner.

\subsubsection{Timing}

Postcoital tests should be performed as close as possible to, but before, the time of ovulation, as determined by clinical criteria, e.g. usual cycle length, basal body temperature, cervical mucus changes, vaginal cytology, serum or urinary luteinizing hormone or estrogen assays, and ovarian ultrasound examination. It is important that the mucus is evaluated in the laboratory at a standard time-between 9 and 14 hours after coitus.

\subsubsection{Instructions for couples}

In preparation for the postcoital test, the couples should be told the most suitable day for the test, and be instructed:

1. to abstain from intercourse, and the man from masturbation, for 2 days before the test;

2. to have vaginal intercourse the night before the test date;

3. to not use any vaginal lubricants during intercourse and the woman should not douche after intercourse (taking a shower, but not a full bath, is permitted);

4. that the woman should report to the clinic for the test the following morning. 


\subsubsection{Procedure}

1. Insert a non-lubricated speculum into the vagina.

2. With a tuberculin syringe (without needle), pipette or polyethylene tube, aspirate as much as possible of the seminal fluid pool in the posterior vaginal fornix.

3. With a different syringe or catheter, aspirate as much mucus as possible from the endocervical canal.

4. Place the mucus sample on a slide and flatten it by applying a coverslip $(22 \mathrm{~mm} \times 22 \mathrm{~mm})$. The depth of this preparation can be standardized by supporting the coverslip with silicone grease or a wax-petroleum jelly mixture (see Box 3.1) containing glass beads of $100 \mu \mathrm{m}$ diameter (Drobnis et al., 1988).

5. Examine the preparation with phase-contrast optics at $\times 400$ magnification.

Note: For reliable results it is crucial that the mucus sample is of good quality and free of blood contaminants.

\section{B ox 3.1 Preparation of a wax-petroleum jelly mixture}

Prepare a wax-petroleum jelly mixture ahead of time. It can be stored at room temperature until ready for use. Melt wax $\left(48-66^{\circ} \mathrm{C}\right.$ melting point) in a beaker and mix in petroleum jelly (approximately one part wax to two parts jelly) with a glass rod. Once the mixture is homogeneous, let it cool down slightly. While it is still warm, draw it into a 3-ml or 5-ml syringe (without a needle). Once the mixture has solidified, load the syringe with an 18-gauge, blunt-end needle.

\subsubsection{The vaginal pool semen sample}

Spermatozoa are usually killed in the vagina within 2 hours. Examine a wet preparation of the vaginal pool sample (see Section 2.4.2) to ensure that semen has been deposited in the vagina.

\subsubsection{The cervical mucus sample}

The number of spermatozoa in the lower part of the cervical canal depends on the length of time since intercourse. Some 2-3 hours after coitus there is a large accumulation of spermatozoa in the lower part of the cervical canal.

The estimate of the number of spermatozoa in the cervical mucus is traditionally based on the number counted per high-power microscope field (see Box 3.2). The concentration of spermatozoa within the mucus should be expressed as the number of spermatozoa per $\mu$ l. 


\section{Box 3.2 Volume observed per high-power field in a 100- $\mu \mathrm{m}$-deep mucus preparation}

The volume of mucus in each microscope field depends on the area of the field $\left(\pi r^{2}\right.$, where $\pi$ is approximately 3.142 and $r$ is the radius of the microscope field) and the depth of the chamber (here $100 \mu \mathrm{m}$ ). The diameter of the microscope field can be measured with a stage micrometer or can be estimated by dividing the diameter of the aperture of the ocular lens by the magnification of the objective lens.

With a $\times 40$ objective and a $\times 10$ ocular of aperture $20 \mathrm{~mm}$, the microscope field has a diameter of approximately $500 \mu \mathrm{m}(20 \mathrm{~mm} / 40)$. In this case $r=250 \mu \mathrm{m}, \mathrm{r}^{2}=$ $62500 \mu \mathrm{m}^{2}, \pi \mathrm{r}^{2}=196375 \mu \mathrm{m}^{2}$ and the volume is $19637500 \mu \mathrm{m}^{3}$ or about $20 \mathrm{nl}$.

Thus, a count of 10 spermatozoa per $\times 400 \mathrm{HPF}$ in a $100-\mu \mathrm{m}$-deep preparation is equivalent to approximately 10 spermatozoa per $20 \mathrm{nl}$ of mucus or 500 spermatozoa per $\mu$ l. However, as the total number of cells counted is low, the sampling error is high. Report the sampling error for 10 cells given in Table 2.2 (approximately $32 \%$ ).

Sperm motility in cervical mucus is graded as follows:

- $P R=$ progressive motility;

- $\mathrm{NP}=$ non-progressive motility;

- $\mathrm{IM}=$ immotile spermatozoa.

The most important indicator of normal cervical function is the presence of any spermatozoa with progressive motility.

\subsubsection{Interpretation}

- The test is negative if no spermatozoa are found in the mucus.

- The presence of any spermatozoa with progressive motility in endocervical mucus 9-14 hours after intercourse argues against significant cervical factors, and sperm autoimmunity in the male or female, as possible causes of infertility (O ei et al., 1995).

- When NP spermatozoa exhibiting a shaking phenomenon are seen, there may be sperm antibodies either in the mucus or on the spermatozoa.

Note: If the initial result is negative or abnormal, the postcoital test should be repeated.

Comment 1: If no spermatozoa are found in the vaginal pool sample, the couple should be asked to confirm that intravaginal ejaculation occurred.

Comment 2: A negative test may be due to incorrect timing. A test performed too early or too late in the menstrual cycle may be negative in a fertile woman. In some women, the test may be positive for only 1 or 2 days during the entire menstrual cycle. When ovulation cannot be predicted with a reasonable degree of accuracy, it may be necessary to repeat the postcoital test several times during a cycle or to perform repeated tests in vitro. 
Comment 3: Repeated negative postcoital tests in cycles with optimal timing are required to establish cervical factors as a possible cause of infertility.

\subsubsection{In-vitro tests}

A detailed assessment of sperm-cervical mucus interaction may be undertaken using in-vitro penetration tests. These tests are usually performed after a negative postcoital test, and are most informative when carried out with cross-over testing using donor semen and donor cervical mucus as controls. They may also be used to assess the significance of a positive sperm antibody test in the male or female partner.

- When the purpose of the sperm-cervical mucus interaction test is to compare the quality of various cervical mucus specimens, a single sample of normozoospermic semen should be used.

- When the purpose is to evaluate the quality of several semen specimens, the same sample of good quality, mid-cycle cervical mucus should be used.

Note: See Appendix 5 for details of collection, storage and evaluation of the characteristics of cervical mucus.

Comment 1: Donor cervical mucus can be obtained at mid-cycle from women who are scheduled for artificial insemination or oocyte retrieval for assisted reproduction. The cervical mucus should be collected prior to insemination, in natural cycles or in cycles in which ovulation has been induced by treatment with gonadotrophins.

Comment 2: Women can be given ethinyl estradiol for 7-10 days to produce estrogenized mucus for testing (see Appendix 5, section A5.2.1).

Comment 3: Women who are receiving clomifene for induction of ovulation should not be used as cervical mucus donors, because of the possible effects of this antiestrogen on the cervix.

- Mid-cycle human cervical mucus should be used.

- In-vitro tests should be done within 1 hour of semen collection, to prevent dehydration or changes in temperature affecting semen quality.

- The $\mathrm{pH}$ of cervical mucus from the endocervical canal should be measured with $\mathrm{pH}$ paper, range 6.0-10.0, in situ or immediately following collection. If the $\mathrm{pH}$ is measured in situ, care should be taken to measure it correctly, since the $\mathrm{pH}$ of exocervical mucus is always lower than that of mucus in the endocervical canal. Care should also be taken to avoid contamination with secretions of the vagina, which have a low $\mathrm{pH}$.

- Spermatozoa are susceptible to changes in $\mathrm{pH}$ of the cervical mucus. Acidic mucus immobilizes spermatozoa, whereas alkaline mucus may enhance motility. Excessive alkalinity of the cervical mucus ( $\mathrm{pH}>8.5)$ may adversely affect the viability of spermatozoa. The optimum $\mathrm{pH}$ value for sperm migration and survival in the cervical mucus is between 7.0 and 8.5 , the $\mathrm{pH}$ range of normal, 
mid-cycle cervical mucus. While a pH value between 6.0 and 7.0 may be compatible with sperm penetration, motility is often impaired below $\mathrm{pH} 6.5$ and sperm-cervical mucus tests are often not performed if the $\mathrm{pH}$ of mucus is less than 7.0.

Note: Surrogate gels, such as bovine cervical mucus or synthetic gels, cannot be regarded as equivalent to human cervical mucus for in-vitro testing of sperm-cervical mucus interaction. However, the use of these materials does provide information on sperm motility within viscous media (Neuwinger et al., 1991; Ivic et al., 2002).

\subsubsection{In-vitro simplified slide test}

\subsubsection{Procedure}

1. Place a drop of cervical mucus on a slide and flatten it by applying a coverslip $(22 \mathrm{~mm} \times 22 \mathrm{~mm})$. The depth of this preparation can be standardized by supporting the coverslip with silicone grease or a wax-petroleum jelly mixture (see Box 3.1) containing glass beads of $100-\mu \mathrm{m}$ diameter (Drobnis et al., 1988).

2. Deposit a drop of semen at each side of the coverslip and in contact with its edge, so that the semen moves under the coverslip by capillary forces. In this way, clear interfaces are obtained between the cervical mucus and the semen.

3. Store the slide horizontally for 30 minutes at $37^{\circ} \mathrm{C}$ in a humid chamber (e.g. on water-saturated filter paper in a covered Petri dish) to prevent drying out.

4. Examine the interface with phase-contrast optics at $\times 400$ magnification.

\subsubsection{Observations}

Observe whether the following features are present:

1. Within a few minutes, finger-like projections (phalanges) of seminal fluid develop and penetrate into the mucus. This is a physical property of the fluids, and can occur even in azoospermic samples (Perloff \& Steinberger, 1963; Moghissi et al., 1964).

2. Most spermatozoa penetrate the phalangeal canal before entering the mucus. In many instances, a single spermatozoon appears to lead a column of spermatozoa into the mucus.

3. Once in the cervical mucus, the spermatozoa fan out and appear to move at random. Some return to the seminal plasma, but most migrate deep into the cervical mucus until they meet resistance from cellular debris or leukocytes.

4. Spermatozoa progress into the mucus for $500 \mu \mathrm{m}$ (i.e. about 10 sperm lengths) from the semen-mucus interface or more.

5. Spermatozoa are motile (note the approximate percentage of motile spermatozoa and whether they are progressively motile). 


\subsubsection{Interpretation}

Interpretation of the simplified slide test is subjective, because it is impossible to standardize the size and shape of the semen-mucus interface in a plain slide preparation. Consequently, it gives only a qualitative assessment of sperm-mucus interaction. Nevertheless, a number of useful observations can be made.

1. Normal result: spermatozoa penetrate into the mucus phase and more than $90 \%$ are motile with definite progression. This suggests that there is no problem with sperm-cervical mucus interaction.

2. Poor result: spermatozoa penetrate into the mucus phase, but most do not progress further than $500 \mu \mathrm{m}$ (i.e. about 10 sperm lengths) from the semenmucus interface. This suggests that there is a problem with sperm-cervical mucus interaction.

3. Abnormal result: either: (1) spermatozoa penetrate into the mucus phase, but rapidly become either immotile or show a "shaking" movement, or (2) spermatozoa do not penetrate the semen-mucus interface. Phalanges may or may not be formed, but the spermatozoa congregate along the semen side of the interface. This suggests the presence of anti-sperm antibodies in the mucus or on the surface of the spermatozoa.

Comment: When an abnormal result is obtained using samples of the couple's semen and mucus, cross-over testing using donor semen and donor cervical mucus can identify whether the semen or the cervical mucus is responsible for the abnormal result.

\subsubsection{Capillary tube test}

The capillary tube test was originally designed by Kremer (1965), and various modifications have since been proposed. The test measures the ability of spermatozoa to penetrate a column of cervical mucus in a capillary tube. The procedure recommended here is based on the original test.

\subsubsection{Equipment}

Various types of capillary tube have been used but flat capillary tubes, $5 \mathrm{~cm}$ long and with a $0.3-\mathrm{mm}$ internal diameter viewing path, are recommended.

A K remer sperm penetration meter (Fig. 3.2) can be constructed in the laboratory as follows.

1. Glue onto a glass slide three reservoirs cut from small, plastic test tubes (radius about $3.5 \mathrm{~mm}$ ).

2. Glue a second glass slide onto the first. The second slide should be $1.5 \mathrm{~cm}$ shorter and positioned at a distance of $5 \mathrm{~mm}$ from the reservoirs. This construction prevents creeping of seminal fluid between the capillary tube and the glass slide.

3. Attach a centimetre scale to the slides. 
Fig. 3.2 The Kremer sperm penetration meter

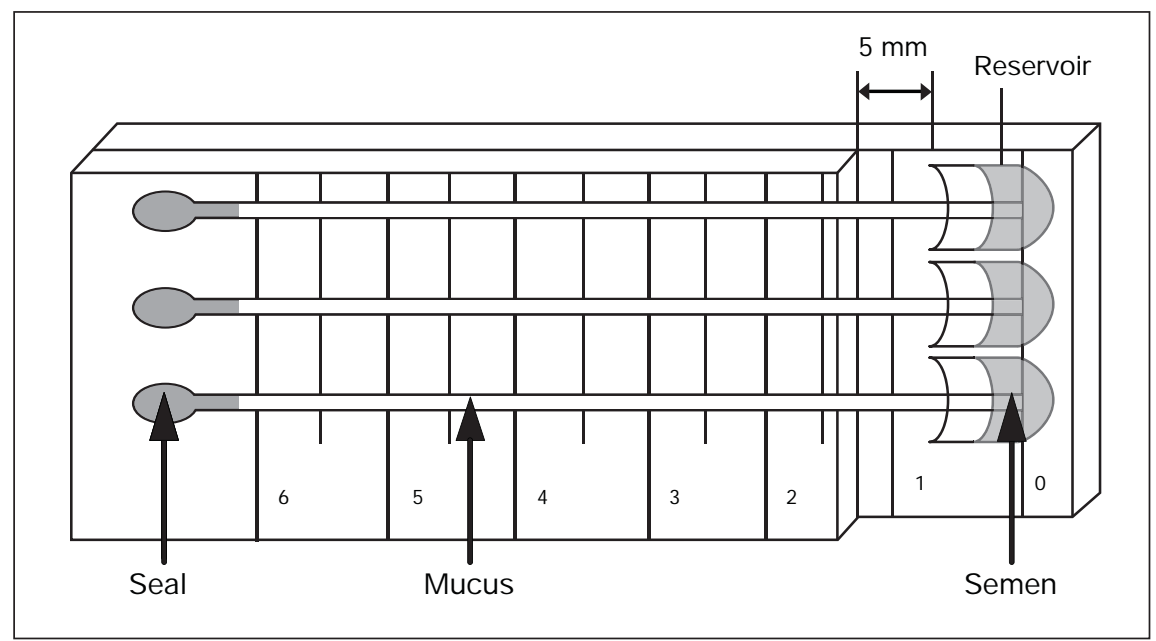

\subsubsection{Procedure}

1. Introduce approximately $100 \mu$ l of liquefied semen, obtained not later than 1 hour after ejaculation, into each of the semen reservoirs.

2. Aspirate cervical mucus into each capillary tube, making sure that no air bubbles are introduced.

3. Seal one end of each tube with a capillary tube sealant, modelling clay or similar material. Enough sealant should be applied so that the mucus column protrudes slightly out of the open end of the tube.

4. Place the open end of the capillary tube on the slide so that it projects about $0.5 \mathrm{~cm}$ into the reservoir containing the semen sample.

5. Store the device horizontally for 2 hours at $37{ }^{\circ} \mathrm{C}$ in a humid chamber (e.g. on water-saturated filter paper in a covered Petri dish) to prevent drying out of the semen and mucus.

6. Examine the capillary tube with phase-contrast optics at $\times 100$ magnification, as outlined in Section 3.3.4.3.

7. Return the device to the $37^{\circ} \mathrm{C}$ incubator and inspect the capillary tubes again after 24 hours for the presence of progressing spermatozoa.

\subsubsection{Observations}

After 2 hours, assess migration distance, penetration density, migration reduction and presence of spermatozoa with forward motility.

1. Migration distance: record the distance from the end of the capillary tube in the semen reservoir to the furthest spermatozoon in the tube.

2. Penetration density: measure this at 1 and $4.5 \mathrm{~cm}$ from the end of the capillary tube in the semen reservoir. At each point, record the mean number of spermatozoa per low-power field (x100 LPF). 
The mean number is obtained from estimates on five adjacent low-power fields, and is expressed as a penetration density rank, as given in Table 3.3. For the classification of the test, the highest sperm penetration density rank is recorded, whether at 1 or $4.5 \mathrm{~cm}$.

Table 3.3 Rank order of sperm penetration density

\begin{tabular}{|c|c|}
\hline Mean number of sperm per LPF & Rank order \\
\hline 0 & 1 \\
\hline $0-5$ & 2 \\
\hline $6-10$ & 3 \\
\hline $11-20$ & 4 \\
\hline $21-50$ & 5 \\
\hline $51-100$ & 6 \\
\hline$>100$ & 7 \\
\hline
\end{tabular}

3. Migration reduction: this is calculated as the decrease in penetration density at $4.5 \mathrm{~cm}$ compared with that at $1 \mathrm{~cm}$. It is expressed as the difference in rank order.

Example 1. Penetration density at $1 \mathrm{~cm}$ is $51-100$ per LPF and at $4.5 \mathrm{~cm}$ is $6-10$. The migration reduction value is 3 (rank order 6 to rank order 3 ) (Table 3.3).

Example 2. Penetration density at $1 \mathrm{~cm}$ is $21-50$ per LPF and at $4.5 \mathrm{~cm}$ is 51-100. The migration reduction value is zero because the penetration density has, in fact, increased (from rank order 5 to rank order 6) (Table 3.3).

4. Spermatozoa with forward motility: determine the presence in the cervical mucus of spermatozoa with forward motility at 2 and 24 hours

\subsubsection{Interpretation}

The results are classified as negative, poor or good according to Table 3.4.

\subsection{Biochemical assays for accessory sex organ function}

Table 3.4 Classification of the capillary tube test results

\begin{tabular}{|c|c|c|c|c|c|c|c|}
\hline $\begin{array}{l}\text { Migration } \\
\text { distance } \\
\text { (cm) }\end{array}$ & & $\begin{array}{l}\text { Highest penetration } \\
\text { density (number of } \\
\text { spermatozoa per } \\
\text { LPF at } 1 \text { or } 4.5 \mathrm{~cm} \text { ) }\end{array}$ & & $\begin{array}{l}\text { Migration reduction } \\
\text { from } 1 \text { to } 4.5 \mathrm{~cm} \\
\text { (decrease in rank } \\
\text { order number) }\end{array}$ & & $\begin{array}{l}\text { Duration of } \\
\text { progressive } \\
\text { movements in } \\
\text { mucus (hours) }\end{array}$ & Classification \\
\hline 1 & & 0 & & - & & - & Negative \\
\hline$<3$ & or & $<10$ & or & $>3$ & or & 2 & Poor \\
\hline 4.5 & and & $>50$ & and & $<3$ & and & $>24$ & Good \\
\hline \multicolumn{7}{|c|}{ All other combinations of test results } & Fair \\
\hline
\end{tabular}


Poor-quality semen may result from testicular production of abnormal spermatozoa, or from post-testicular damage to spermatozoa in the epididymis or the ejaculate from abnormal accessory gland secretions. Secretions from accessory glands can be measured to assess gland function, e.g. citric acid, zinc, $\gamma$-glutamyl transpeptidase and acid phosphatase for the prostate; fructose and prostaglandins for the seminal vesicles; free L-carnitine, glycerophosphocholine (GPC) and neutral $\alpha$-glucosidase for the epididymis.

An infection can sometimes cause a decrease in the secretion of these markers, but the total amount of markers present may still be within the normal range. An infection can also cause irreversible damage to the secretory epithelium, so that even after treatment secretion may remain low (Cooper et al., 1990a; von der Kammer et al., 1991).

- Secretory capacity of the prostate. The amount of zinc, citric acid (Möllering \& Gruber, 1966) or acid phosphatase (Heite \& Wetterauer, 1979) in semen gives a reliable measure of prostate gland secretion, and there are good correlations between these markers. A spectrophotometric assay for zinc is described in Section 3.4.1.

- Secretory capacity of the seminal vesicles. Fructose in semen reflects the secretory function of the seminal vesicles. A spectrophotometric method for its estimation is described in Section 3.4.2.

- Secretory capacity of the epididymis. L-Carnitine, GPC and neutral $\alpha$-glucosidase are epididymal markers used clinically. Neutral $\alpha$-glucosidase has been shown to be more specific and sensitive for epididymal disorders than L-carnitine and GPC (Cooper et al., 1990a). There are two isoforms of $\alpha$-glucosidase in the seminal plasma: the major, neutral form originates solely from the epididymis, and the minor, acidic form, mainly from the prostate. A simple spectrophotometric assay for neutral $\alpha$-glucosidase is described in Section 3.4.3.

Comment: The total content of any accessory gland secretion in the ejaculate reflects the overall secretory function of that gland (Eliasson, 1975). This is obtained by multiplying the accessory gland marker concentration by the volume of the whole ejaculate.

\subsubsection{Measurement of zinc in seminal plasma}

\subsubsection{Background}

A kit for measurement of serum zinc by spectrophotometric assay is commercially available and can be adapted for semen. The method described below is based on that of J ohnsen \& Eliasson (1987), modified for the use of a 96 -well plate reader with sensitivity $4 \mu \mathrm{mol} / \mathrm{l}$ (Cooper et al., 1991). The volumes of semen and reagents can be proportionally adjusted for spectrophotometers using 3-ml or 1-ml cuvettes. The appropriate corrections must be made in calculating the results. 


\subsubsection{Principle}

The compound 2- (5-bromo-2-pyridylazo)-5- (N-propyl- N-sulfopropylamino)-phenol (5-Br-PAPS) binds with zinc, producing a change in colour.

5-Br-PAPS $+\mathrm{Zn}^{2+} \rightarrow$ 5-Br-PAPS-Zn complex, which absorbs light of wavelength $560 \mathrm{~nm}$.

\subsubsection{Reagents}

1. A kit for the estimation of zinc in serum is commercially available. Use only colour reagent $A(2 \times 60 \mathrm{ml}$ bottles) and colour reagent $B(1 \times 30 \mathrm{ml}$ bottle).

2. Zinc standard $(100 \mu \mathrm{mol} / \mathrm{l})$ : dissolve $0.144 \mathrm{~g}$ of zinc sulfate $\mathrm{ZnSO}_{4} \cdot 7 \mathrm{H}_{2} \mathrm{O}$ in $50 \mathrm{ml}$ of purified water and dilute this 100 times by adding $1 \mathrm{ml}$ to $99 \mathrm{ml}$ of purified water. Store frozen at $-20^{\circ} \mathrm{C}$.

3. Standard curve: dilute the $100 \mu \mathrm{mol} / /$ zinc standard, prepared in step 2, with purified water to yield five additional standards of $80,60,40,20$ and $10 \mu \mathrm{mol} / \mathrm{l}$.

4. Colour reagent: mix 4 parts of colour reagent $A$ with 1 part of colour reagent $B$ (about $25 \mathrm{ml}$ is needed for one 96 -well plate). This chromogen solution is stable for 2 days at room temperature or 1 week at $4{ }^{\circ} \mathrm{C}$.

5. Frozen internal quality-control pools of seminal plasma (see Section 3.4.1.4, step 1).

\subsubsection{Procedure}

1. Centrifuge the semen sample remaining after semen analysis for 10 minutes at $1000 \mathrm{~g}$. Decant and store the sperm-free seminal plasma at $-20^{\circ} \mathrm{C}$ until analysis. Sperm-free seminal plasma can be pooled with other samples to provide a standard for internal quality control in future assays.

2. Thaw the sperm-free seminal plasma and mix well on a vortex mixer. Also thaw and mix an aliquot of pooled seminal plasma for internal quality control.

3. Prepare dilutions of each sample of seminal plasma in replicate: to $300 \mu \mathrm{l}$ of purified water in each of two 1.5 - $\mathrm{ml}$ tubes, add $5 \mu$ l of seminal plasma (with a positive displacement pipette) and mix by vortexing for 5 seconds.

4. Add replicate 40- $\mu$ l aliquots of the diluted seminal plasma samples from step 3 to a 96 -well plate. Include replicate blanks ( $40 \mu$ l of purified water) and $40-\mu$ l replicates of each of the standards.

5. Add $200 \mu$ l of colour reagent to each well and mix for 5 minutes on a 96 -well plate shaker.

6. Read the plate at $560 \mathrm{~nm}$ wavelength, using the water blank to set the zero.

\subsubsection{Calculation}

1. Read the concentration of zinc in the sample from the standard curve ( $\mathrm{mmol} / \mathrm{l})$ by comparing the absorbance values. 
2. Reject results that are above the top standard, and re-assay these samples at greater dilution (use purified water to dilute).

3. Multiply the results by the dilution factor of 61 ( $5 \mu$ l of seminal plasma diluted with $300 \mu$ l of water) to obtain the concentration of zinc $(\mathrm{mmol} / \mathrm{l})$ in undiluted seminal plasma.

4. Replicates should agree within $10 \%$, i.e. (difference between estimates/mean of estimates) $\times 100 \leq 10 \%$. If they do not, repeat the assay on two new aliquots of seminal plasma.

5. Multiply the zinc concentration by the whole volume of semen $(\mathrm{ml})$ to obtain the total zinc content $(\mu \mathrm{mol})$ of the ejaculate.

\subsubsection{Lower reference limit}

The lower reference limit for zinc is $2.4 \mu \mathrm{mol}$ per ejaculate (Cooper et al., 1991 and unpublished data from TG Cooper).

\subsubsection{Measurement of fructose in seminal plasma}

\subsubsection{Background}

The method described below is based on that of Karvonen \& Malm (1955), modified for use with a 96-well plate reader with sensitivity $74 \mu \mathrm{mol} / \mathrm{l}$ (Cooper et al., 1990a). The volumes of semen and reagents can be proportionally adjusted for spectrophotometers using 3- $\mathrm{ml}$ or 1 - $\mathrm{ml}$ cuvettes. The appropriate corrections must be made in calculating the results.

\subsubsection{Principle}

Under the influence of heat and low $\mathrm{pH}$, fructose forms a coloured complex with indole.

Fructose + indole $\stackrel{\text { heat }+ \text { acid }}{\longrightarrow}$ complex, which absorbs light of wavelength $470 \mathrm{~nm}$.

\subsubsection{Reagents}

A kit for the estimation of fructose in seminal plasma is commercially available. Alternatively, prepare the following reagents.

1. Deproteinizing agent $1\left(63 \mu \mathrm{mol} / \mathrm{ZnSO}_{4}\right)$ : dissolve $1.8 \mathrm{~g}$ of $\mathrm{ZnSO}_{4} \cdot 7 \mathrm{H}_{2} \mathrm{O}$ in $100 \mathrm{ml}$ of purified water.

2. Deproteinizing agent $2(1 \mathrm{~mol} / \mathrm{l} \mathrm{NaOH})$ : dissolve $0.4 \mathrm{~g}$ of $\mathrm{NaOH}$ in $100 \mathrm{ml}$ of purified water.

3. Colour reagent (indole $2 \mu \mathrm{mol} / \mathrm{l}$ in benzoate preservative $16 \mu \mathrm{mol} / \mathrm{l}$ ): dissolve $200 \mathrm{mg}$ of benzoic acid in $90 \mathrm{ml}$ of purified water by shaking it in a water bath at $60{ }^{\circ} \mathrm{C}$. Dissolve $25 \mathrm{mg}$ of indole in this and make up to $100 \mathrm{ml}$ with purified water. Filter $\left(0.45-\mu \mathrm{m}\right.$ pore size) and store at $4{ }^{\circ} \mathrm{C}$.

4. Fructose standard $(2.24 \mathrm{mmol} / \mathrm{l})$ : dissolve $40 \mathrm{mg}$ of $\mathrm{D}$-fructose in $100 \mathrm{ml}$ of purified water. Store at $4{ }^{\circ} \mathrm{C}$ or freeze in aliquots. 
5. Standard curve: dilute the $2.24 \mathrm{mmol} / \mathrm{l}$ standard with purified water to yield four additional standards of $1.12,0.56,0.28$ and $0.14 \mathrm{mmol} / \mathrm{l}$.

6. Frozen internal quality-control pools of seminal plasma (see Section 3.4.2.4, step 1).

\subsubsection{Procedure}

1. Centrifuge the semen sample remaining after semen analysis for 10 minutes at $1000 \mathrm{~g}$. Decant and store the sperm-free seminal plasma at $-20^{\circ} \mathrm{C}$ until analysis. Sperm-free seminal plasma can be pooled with other samples to provide a standard for internal quality control in future assays.

2. Thaw the sperm-free seminal plasma and mix well on a vortex mixer. Also thaw and mix an aliquot of pooled seminal plasma for internal quality control.

3. Prepare dilutions of each seminal plasma sample in replicate: to $50 \mu \mathrm{l}$ of purified water in each of two $1.5-\mathrm{ml}$ tubes, add $5 \mu$ l of seminal plasma (with a positive displacement pipette) and mix.

4. Deproteinize: to the $55 \mu \mathrm{l}$ of diluted sample add $12.5 \mu \mathrm{l}$ of $63 \mu \mathrm{mol} / \mathrm{I} \mathrm{ZnSO}_{4}$ and $12.5 \mu \mathrm{l}$ of $0.1 \mathrm{~mol} / \mathrm{l} \mathrm{NaOH}$ and mix. Allow to stand for 15 minutes at room temperature, then centrifuge at $8000 \mathrm{~g}$ for 5 minutes.

5. Transfer $50 \mu$ l of supernatant from each sample to a test tube. Include replicate blanks ( $50 \mu$ l of water) and $50-\mu$ l replicates of each standard.

6. Add $50 \mu$ l of indole reagent to each tube and mix.

7. Add $0.5 \mathrm{ml}$ of concentrated $(32 \% \mathrm{v} / \mathrm{v})$ hydrochloric acid $(\mathrm{HCl})$ to each sample, cover with self-sealing, mouldable laboratory film and mix carefully in a fume cupboard.

8. Heat for 20 minutes at $50{ }^{\circ} \mathrm{C}$ in a water bath. Mix and cool in ice-water for 15 minutes.

9. Carefully transfer $250 \mu$ l with a positive-displacement pipette to a 96 -well plate in a fume cupboard.

10. Seal the 96-well plate with transparent adhesive laboratory film to protect the spectrophotometer from the acid.

11. Read the plate at $470 \mathrm{~nm}$ wavelength, using the water blank to set the zero.

\subsubsection{Calculation}

1. Read the concentration of fructose in the sample from the standard curve ( $\mathrm{mmol} / \mathrm{l}$ ) by comparing absorbance values.

2. Reject results that are above the top standard, and re-assay these samples at greater dilution (use purified water to dilute).

3. Multiply the results for each sample by the dilution factor of 16 ( $5 \mu$ l of seminal plasma diluted with $75 \mu$ l of water and deproteinizing agents) to obtain the concentration of fructose $(\mathrm{mmol} / \mathrm{l})$ in undiluted seminal plasma. 
4. Replicates should agree within $10 \%$, i.e. (difference between estimates/mean of estimates) $\times 100 \leq 10 \%$. If they do not, repeat the assay on two new aliquots of semen.

5. Multiply the fructose concentration by the whole volume of semen $(\mathrm{ml})$ to obtain the total fructose content ( $\mu \mathrm{mol})$ of the ejaculate.

\subsubsection{Lower reference limit}

The lower reference limit for fructose is $13 \mu \mathrm{mol}$ per ejaculate (Cooper et al., 1991 and unpublished data from TG Cooper).

Comment: Low fructose in semen is characteristic of ejaculatory duct obstruction, bilateral congenital absence of the vas deferens (de la Taille et al., 1998; Daudin et al., 2000; von Eckardstein et al., 2000), partial retrograde ejaculation and androgen deficiency.

\subsubsection{Measurement of neutral $\alpha$-glucosidase in seminal plasma}

\subsubsection{Background}

Seminal plasma contains both a neutral $\alpha$-glucosidase isoenzyme, which originates in the epididymis, and an acid isoenzyme contributed by the prostate. The latter can be selectively inhibited by sodium dodecyl sulfate (SDS) (Paquin et al., $1984)$ to permit measurement of the neutral $\alpha$-glucosidase, which reflects epididymal function. Accounting for non-glucosidase-related substrate breakdown, by using the inhibitor castanospermine, makes the assay more sensitive. The method described below is for use with a 96 -well plate reader with sensitivity $1.9 \mathrm{mU} / \mathrm{ml}$ (Cooper et al., 1990b). The volumes of semen and reagents can be proportionally adjusted for spectrophotometers with 3- $\mathrm{ml}$ or 1-ml cuvettes. The appropriate corrections must be made in calculating the results.

\subsubsection{P rinciple}

Glucosidase converts the synthetic glucopyranoside substrate to p-nitrophenol, which turns yellow on addition of sodium carbonate.

$$
\begin{aligned}
& \text { p-nitrophenol- } \stackrel{\alpha \text {-glucosidase }}{\longrightarrow} \text { p-nitrophenol } \stackrel{\mathrm{Na}_{2} \mathrm{CO}_{3}}{\longrightarrow} \text { complex, which } \\
& \alpha \text {-glucopyranoside absorbs light of } \\
& \text { wavelength } 405 \mathrm{~nm}
\end{aligned}
$$

\subsubsection{Reagents}

A kit for the estimation of epididymal neutral $\alpha$-glucosidase in semen is commercially available. Only kits including SDS and castanospermine are recommended for measurement of this enzyme in semen. Alternatively, prepare the following reagents.

1. Buffer 1 (0.2 mol/l phosphate, $\mathrm{pH} 6.8)$ : dissolve $4.56 \mathrm{~g} \mathrm{~K}_{2} \mathrm{HPO}_{4} \cdot 3 \mathrm{H}_{2} \mathrm{O}$ in $100 \mathrm{ml}$ of purified water. Dissolve $2.72 \mathrm{~g}$ of $\mathrm{KH}_{2} \mathrm{PO}_{4}$ in a separate $100 \mathrm{ml}$ aliquot of purified water. Mix approximately equal volumes of each until the $\mathrm{pH}$ is 6.8 . 
2. Buffer 2: dissolve $1 \mathrm{~g}$ of SDS in $100 \mathrm{ml}$ of buffer 1 . SDS will precipitate on storage at $4{ }^{\circ} \mathrm{C}$, but redissolves on gentle warming.

3. Colour reagent 1 (for stopping the reaction, $0.1 \mathrm{~mol} / /$ sodium carbonate): dissolve $6.20 \mathrm{~g}$ of $\mathrm{Na}_{2} \mathrm{CO}_{3} \cdot \mathrm{H}_{2} \mathrm{O}$ in $500 \mathrm{ml}$ of water.

4. Colour reagent 2: dissolve $0.1 \mathrm{~g}$ of SDS in $100 \mathrm{ml}$ of colour reagent 1 .

5. Substrate $p$-nitrophenol glucopyranoside (PNPG) $(5 \mathrm{mg} / \mathrm{ml})$ : dissolve $0.1 \mathrm{~g}$ of PNPG in $20 \mathrm{ml}$ of buffer 2 and warm the solution on a hotplate at about $50{ }^{\circ} \mathrm{C}$ with stirring for about 10 minutes. A few crystals may remain undissolved. The solution should be kept at $37{ }^{\circ} \mathrm{C}$ during use. Make a fresh solution for each assay.

6. Glucosidase inhibitor for semen blanks (castanospermine, $10 \mathrm{mmol} / \mathrm{l}$ ): dissolve $18.9 \mathrm{mg}$ of castanospermine in $10 \mathrm{ml}$ of purified water. Dilute this 10 -fold in purified water to give a $1 \mathrm{mmol} / \mathrm{l}$ working solution. Freeze approximately $1-\mathrm{ml}$ aliquots at $-20^{\circ} \mathrm{C}$.

7. Standard curve of product $\mathrm{p}$-nitrophenol (PNP) $(5 \mathrm{mmol} / \mathrm{l})$ : dissolve $69.5 \mathrm{mg}$ of PNP in $100 \mathrm{ml}$ of purified water, warming the solution if necessary. Store at $4{ }^{\circ} \mathrm{C}$ in the dark in an aluminium foil-covered or brown glass bottle. Make up a fresh standard solution every 3 months.

8. Prepare a standard curve (within the last hour of incubation): place $400 \mu \mathrm{l}$ of $5 \mathrm{mmol} / \mathrm{l}$ stock PNP in a $10-\mathrm{ml}$ volumetric flask and make up to $10 \mathrm{ml}$ with colour reagent $2(200 \mu \mathrm{mol} / \mathrm{l})$. Dilute the $200 \mu \mathrm{mol} / \mathrm{l}$ standard with colour reagent 2 to yield four additional standards of 160, 120, 80 and $40 \mu \mathrm{mol} / \mathrm{I} \mathrm{PNP.}$

9. Frozen internal quality-control pools of seminal plasma (see Section 3.4.3.4, step 1).

\subsubsection{Procedure}

1. Centrifuge the semen sample remaining after analysis for 10 minutes at $1000 \mathrm{~g}$. Decant and store the sperm-free seminal plasma at $-20{ }^{\circ} \mathrm{C}$ until analysis. Sperm-free seminal plasma can be pooled with other samples to provide a quality control pool as an internal standard for future assays.

2. Thaw the sperm-free seminal plasma and mix well on a vortex mixer. Also thaw and mix an aliquot of pooled seminal plasma for internal quality control.

3. Place replicate samples of $15 \mu$ l of seminal plasma in each of two $1.5-\mathrm{ml}$ tubes using a positive displacement pipette. Include replicate blanks ( $15 \mu \mathrm{l}$ of water) and quadruplicate $15-\mu$ internal quality-control samples from semen pools.

4. To two of the internal quality-control samples add $8 \mu \mathrm{l}$ of $1 \mathrm{mmol} / \mathrm{l}$ castanospermine to provide the seminal plasma blank value.

5. Add $100 \mu$ l of PNPG substrate solution, at about $37^{\circ} \mathrm{C}$, to each tube.

6. Vortex each tube and incubate at $37{ }^{\circ} \mathrm{C}$ for 2 hours (exact temperature and time control are crucial). 
7. Stop incubation after 2 hours by adding $1 \mathrm{ml}$ of colour reagent 1 and mix.

8. Transfer $250 \mu$ l of samples and standards to the 96 -well plate.

9. Read the plate in a 96 -well plate reader at $405 \mathrm{~nm}$ wavelength within $60 \mathrm{~min}$ utes, using the water blank to set the zero.

\subsubsection{Calculation}

1. Read the concentration of PNP produced by the sample from the standard curve $(\mu \mathrm{mol} / \mathrm{l})$ by comparing absorbance values.

2. Reject samples that lie above the top standard and re-assay these samples after dilution (use buffer 1 to dilute).

3. Multiply by the correction factor (0.6194; see Note) to obtain the activity of neutral glucosidase in undiluted seminal plasma (IU/I).

4. Subtract the activity (IU/I) of the castanospermine seminal plasma blank from each sample to obtain the corrected (glucosidase-related) activity.

5. Replicates should agree within $10 \%$, i.e. (difference between estimates/mean of estimates) $\times 100 \leq 10 \%$. If they do not, repeat the assay on two new aliquots of seminal plasma.

6. Multiply the corrected glucosidase activity by the whole volume of semen (ml) to obtain the glucosidase activity $(\mathrm{mU})$ of the ejaculate.

Note: One international unit (IU) of glucosidase activity is defined as the production of $1 \mu \mathrm{mol}$ of product (PNP) per minute at $37^{\circ} \mathrm{C}$. In this assay the activity is derived from $15 \mu$ l of seminal plasma in a total volume of $1.115 \mu$ l over 120 minutes, so the correction factor is $(1115 / 15) / 120=0.6194$.

\subsubsection{Reference limit}

The lower reference limit for neutral $\alpha$-glucosidase is $20 \mathrm{mU}$ per ejaculate (Cooper et al., 1991 and unpublished data from TG Cooper).

\subsection{Computer-aided sperm analysis}

\subsubsection{Introduction}

Until recently, it was not feasible to measure sperm concentration by computeraided sperm analysis (CASA) because of difficulties in distinguishing spermatozoa from particulate debris (ESHRE, 1998). However, advances in technology, particularly in the use of fluorescent DNA stains and tail-detection algorithms, may now allow sperm concentration-and hence the concentration of progressively motile spermatozoa - to be determined (Zinaman et al., 1996; Garrett et al., 2003). Provided that adequate care is taken in preparing specimens and using the instrument, CASA can now be used for some routine diagnostic applications. Qualitycontrol procedures are necessary to establish and maintain a high standard of instrument operation (see Chapter 7). 
Several manufacturers produce CASA systems. These machines are capable of measuring sperm motility and kinematics, and some can also be used to estimate sperm concentration. A few have semi-automated morphology modules. CASA, including assessment of motility, concentration and morphology, has two advantages over manual methods: it has high precision and it provides quantitative data on the kinematic parameters of spermatozoa (forward progression and hyperactivated motility, characteristic of capacitated cells).

Some studies have suggested that CASA estimates of concentration and movement characteristics of progressively motile spermatozoa are significantly related to fertilization rates in vitro and in vivo, as well as to time to conception (Liu et al., 1991a; Barratt et al., 1993; Irvine et al., 1994; Krause, 1995; Donnelly et al., 1998; Larsen et al., 2000; Garrett et al., 2003; Shibahara et al., 2004). The use of CASA to measure sperm motility and concentration is described in Sections 3.5.2 and 3.5.3, respectively, while Section 3.5.4 contains a commentary on the status of computer-aided morphological analysis.

\subsubsection{Use of CASA to assess sperm motility}

CASA machines are best used for kinematic analysis of spermatozoa, as they can detect motile cells. Estimates of percentage motility may be unreliable, as they depend on determining the number of immotile spermatozoa, and debris may be confused with immotile spermatozoa.

Many factors affect the performance of CASA instruments, e.g. sample preparation, frame rate, sperm concentration and counting-chamber depth (Davis \& Katz, 1992; Mortimer, 1994a, b; Kraemer et al., 1998). Nevertheless, reliable and reproducible results can be obtained if appropriate procedures are followed (Davis \& Katz, 1992). Guidelines on the use of CASA (Mortimer et al., 1995; ESHRE, 1998) should be consulted.

In using CASA to obtain movement parameters, the tracks of at least 200 motile spermatozoa per specimen should be analysed. This implies that many more spermatozoa will need to be detected. If the spermatozoa are to be categorized by type of motion, or if other analyses of variability within a specimen are planned, the tracks of at least 200 , and if possible 400 , motile spermatozoa will be needed. The number of spermatozoa analysed in each specimen should be standardized.

The CASA instrument should be linked to computer software that permits data organization and statistical analysis. The distributions of many of the movement parameters are not Gaussian; the median, rather than the mean, is therefore more appropriate as a summary of the central tendency of each variable. The measurements on single spermatozoa may need to be mathematically transformed before certain statistical analyses are done.

\subsubsection{Procedure}

Each CASA instrument must be correctly set up for its anticipated use in order to ensure optimum performance. The manufacturers indicate suitable settings, but users should check that the instrument is performing to the required degree of repeatability and reliability. Use of appropriate quality control materials, e.g. video 
recordings, is essential (see Appendix 7, section A7.5). Several authors have discussed CASA settings in a general context (Davis \& Katz, 1992; Mortimer, 1994b; ESHRE, 1998).

\subsubsection{Preparing the samples}

Semen samples for CASA should be collected and prepared as outlined in Chapter 2 . The CASA system must maintain the specimen at $37^{\circ} \mathrm{C}$, because sperm motion is sensitive to temperature. Motility characteristics and sperm concentration can be assessed in undiluted semen. Sperm motility can be assessed on samples with sperm concentrations between $2 \times 10^{6}$ per $\mathrm{ml}$ and $50 \times 10^{6}$ per $\mathrm{ml}$ (Garrett et al., 2003).

In samples with high sperm concentrations (i.e. greater than $50 \times 10^{6}$ per $\mathrm{ml}$ ), collisions may occur with high frequency and are likely to induce errors. Such samples should be diluted, preferably with seminal plasma from the same man.

1. Centrifuge a portion of the sample at $16000 \mathrm{~g}$ for 6 minutes to produce spermfree seminal plasma.

2. Dilute the original semen sample with the sperm-free seminal plasma to bring the concentration below $50 \times 10^{6}$ per $\mathrm{ml}$.

Disposable counting chambers, $20 \mu \mathrm{m}$ deep, give reliable results. This is a dualchamber system; both chambers should be filled and assessed. Several representative fields should be examined: reading six fields per chamber (12 fields in total) usually gives reliable results. At least 200 spermatozoa should be assessed in each chamber. The same principles of quality control apply as for standard estimations of motility (see Section 2.5.2). Samples can be analysed either directly or from a video recording. Analysing video-recordings (from videotape, CD-ROM or DVD) allows better standardization and implementation of quality assurance procedures (see Appendix 7, section A7.5). The manufacturer will usually recommend the type of recording device to be used and the illumination setting needed for maximum contrast between sperm heads and background.

There is some disagreement regarding the time for which spermatozoa should be followed to achieve accurate results, but a minimum of 1 second should be sufficient for the basic CASA measurements (Mortimer, 1994b).

\subsubsection{CASA terminology}

Some standard terminology for variables measured by CASA systems is illustrated in Fig. 3.3.

1. VCL, curvilinear velocity $(\mu \mathrm{m} / \mathrm{s})$. Time-averaged velocity of a sperm head along its actual curvilinear path, as perceived in two dimensions in the microscope. A measure of cell vigour.

2. VSL, straight-line (rectilinear) velocity $(\mu \mathrm{m} / \mathrm{s})$. Time-averaged velocity of a sperm head along the straight line between its first detected position and its last. 
Fig. 3.3 Standard terminology for variables measured by CASA systems

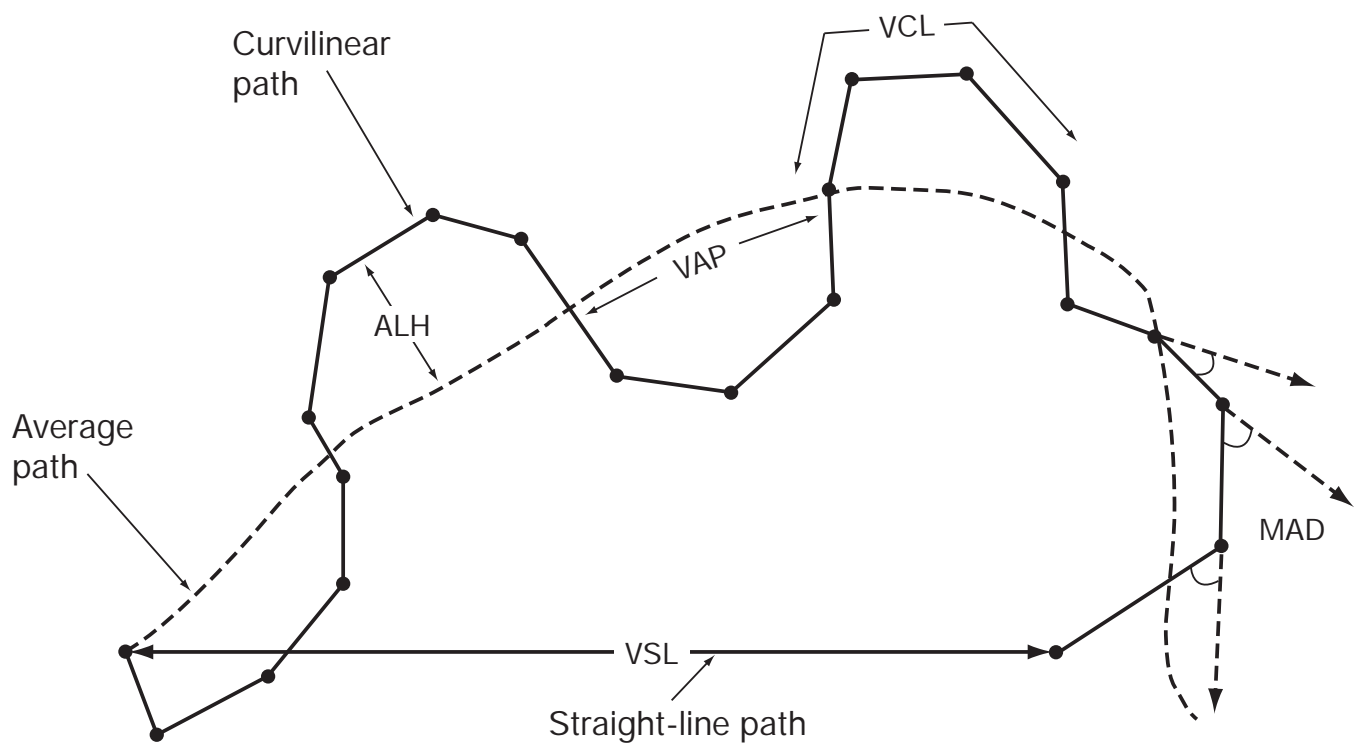

3. VAP, average path velocity $(\mu \mathrm{m} / \mathrm{s})$. Time-averaged velocity of a sperm head along its average path. This path is computed by smoothing the curvilinear trajectory according to algorithms in the CASA instrument; these algorithms vary between instruments, so values may not be comparable among systems.

4. ALH, amplitude of lateral head displacement $(\mu \mathrm{m})$. Magnitude of lateral displacement of a sperm head about its average path. It can be expressed as a maximum or an average of such displacements. Different CASA instruments compute ALH using different algorithms, so values may not be comparable among systems.

5. LIN, linearity. The linearity of a curvilinear path, VSL/VCL.

6. WOB, wobble. A measure of oscillation of the actual path about the average path, VAP/VCL.

7. STR, straightness. Linearity of the average path, VSL/VAP.

8. BCF, beat-cross frequency $(\mathrm{Hz})$. The average rate at which the curvilinear path crosses the average path.

9. MAD, mean angular displacement (degrees). The time-averaged absolute values of the instantaneous turning angle of the sperm head along its curvilinear trajectory.

Note: Different CASA instruments use different mathematical algorithms to compute many of these movement variables. The comparability of measurements across all instruments is not yet known. 


\subsubsection{Use of CASA to estimate sperm concentration}

The use of fluorescent DNA stains with CASA allows the concentration of motile sperm and percentage motility to be determined accurately, but scrupulous adherence to technique is required (Garrett et al., 2003). For example, if disposable chambers are used, it is important to assess the sample at several different distances from the site of loading the chamber as the distribution of spermatozoa throughout the chamber will be non-uniform (Douglas-Hamilton et al., 2005b). Validation against a haemocytometer is essential.

Sperm concentrations of between $2 \times 10^{6}$ per $\mathrm{ml}$ and $50 \times 10^{6}$ per $\mathrm{ml}$ can be measured (Garrett et al., 2003). Samples with a sperm concentration higher than $50 \times 10^{6}$ per $\mathrm{ml}$ will need to be diluted (see Section 3.5.2.2).

Comment: The CASA instrument detects and counts fluorescent sperm heads. Without microscopic evaluation, there is no way of knowing if the spermatozoa are intact (i.e. the head is attached to a tail).

\subsubsection{Computer-aided sperm morphometric assessment}

Image analysis has the potential to bring about major advances in quantification, objectivity and reproducibility in the assessment of sperm morphology. Commercial systems are available for quantifying the morphology of the sperm head and midpiece, and possibly the principal piece. However, tail defects affecting motility can be more directly assessed by using CASA to measure motility and motion. CASA systems generally classify the sperm head and midpiece as normal or abnormal and give the mean and standard deviation or median for head and midpiece dimensions, head ellipticity and regularity, and a stain-dependent measurement of the acrosome area.

Automated systems have the potential for greater objectivity, precision and reproducibility than manual systems (Menkveld et al., 1990). Precision and reproducibility can be less than 7\% (Garrett \& Baker, 1995), which is superior to manual evaluation by an experienced technician. The reproducibility and accuracy of the results of computer-aided sperm morphometric assessment (CASMA) can, however, be compromised by methodological inconsistencies, such as focus, illumination, sample preparation and staining (Lacquet et al., 1996; Menkveld et al., 1997) and by technical difficulties in correctly differentiating sperm heads from seminal debris, particularly when sperm concentration is low (Garrett \& Baker, 1995; Menkveld et al., 1997; Coetzee et al., 1999a, b). The nature of automated evaluation means that there is no way to compensate for preparation defects and artefacts. Thus small differences in background shading relative to cell staining can result in incorrect classification or an inability to identify the cell as a spermatozoon, with a consequent bias in the results.

As with manual morphology assessment, procedures and instruments must be standardized and quality control maintained to ensure comparable and reliable results. Semen may be treated as in Section 2.13.2.4 to reduce background for 
CASMA recordings. If the sperm concentration is low $\left(<2 \times 10^{6}\right.$ per $\left.\mathrm{ml}\right)$, samples will need to be concentrated by centrifugation, as described in Section 2.13.2.2.

Note: Centrifugation may affect sperm morphology and its use must be recorded.

Two studies have reported significant relationships between CASMA results and fertility endpoints. Coetzee et al. (1999c) found auto mated normal sperm morphology outcomes to be significant predictors of both fertilization rates in vitro and pregnancy. Garrett et al. (2003) found that the percentage of spermatozoa in semen that exhibited head morphology characteristic of those that are bound to the zona pellucida ("zona-preferred", \% Z) to gether with straight-line velocity (VSL) in semen were significantly and independently related to natural pregnancy rates in a large group of subfertile couples. The relationships of both $\% \mathrm{Z}$ and VSL with fertility appeared to be continuous, and no threshold value was identified above which there was no further increase in pregnancy rate. More studies of fertility outcomes in large populations are required to refine the application of CASA to measuring sperm morphology.

Automated systems may have a role in providing data for quality control systems, but more research is needed to demonstrate their benefit for clinics. 


\section{CHAPTER $4 \quad$ Research procedures}

When tests of sperm function are to be performed, it is critical that the spermatozoa are separated from the seminal plasma within 1 hour of ejaculation, to limit any damage to spermatozoa from products of non-sperm cells. As our knowledge of the molecular mechanisms regulating sperm function increases, so too will opportunities for the development of new diagnostic tests. For example, recent data emphasize the importance of nuclear DNA compaction and integrity in determining the functional competence of human spermatozoa. Emerging evidence suggests associations between DNA integrity and chromatin organization in spermatozoa and fertility (Sakkas et al., 1998; Aitken \& Krausz, 2001; Virro et al., 2004).

Similarly, advances in our understanding of the signal transduction pathways regulating sperm function will have implications for the development of diagnostic tests capable of generating detailed information on the precise nature of the processes that are defective in the spermatozoa of infertile men. In order to gain deeper insights into the biological basis of male infertility, a battery of functional tests has been developed aimed at assessing the competence of human spermatozoa to fulfil the fundamental processes essential to conception: binding to the zona pellucida, acrosomal exocytosis, and fusion with the vitelline membrane of the oocyte.

\subsection{Reactive oxygen species}

\subsubsection{Introduction}

The excessive generation of reactive oxygen species (ROS) and the presence of high activities of cytoplasmic enzymes, such as creatine phosphokinase, may reflect abnormal spermatozoa with excess residual cytoplasm in the midpiece (Rao et al., 1989; Gomez et al., 1996; Aitken et al., 2004).

Reactive oxygen species are metabolites of oxygen and include the superoxide anion, hydrogen peroxide, hydroxyl and hydroperoxyl radicals, and nitric oxide. When present in excess, they can initiate pathological changes by inducing oxidative damage to cellular lipids, proteins and DNA (Griveau \& Le Lannou, 1997; Aitken et al., 2003; Henkel et al., 2004). Most cells are equipped with either enzymatic antioxidant systems (superoxide dismutase, glutathione peroxidase and catalase) or non-enzymatic antioxidant systems (uric acid, ascorbic acid, $\alpha$-tocopherol), and when these defences are overwhelmed, sperm function is impaired (Agarwal et al., 2004).

In the human ejaculate, reactive oxygen species are produced by both spermatozoa (Aitken \& Clarkson, 1987; Alvarez et al., 1987; Iwasaki \& Gagnon, 1992) and leukocytes (Aitken $\&$ West, 1990). Seminal plasma possesses free radical antioxidant scavengers and antioxidant enzymes, which may be deficient in some men (J ones et al., 1979; S mith et al., 1996). Thus the removal of seminal plasma during the preparation of spermatozoa for assisted conception (see Chapter 5) may render these cells vulnerable to oxidative attack. High ROS production may cause peroxidative damage and loss of sperm function, as well as DNA damage in both the nuclear and mitochondrial genomes (Sawyer et al., 2003). Sperm sur- 
vival assays are frequently used to assess the quality of human spermatozoa. The results of such assays are highly correlated with the lipid peroxidation status of the spermatozoa (Gomez et al., 1998).

A chemiluminescent procedure, employing probes such as luminol or lucigenin, may be used to measure ROS production and the redox activity of human spermatozoa.

\subsubsection{Measurement of reactive oxygen species generated by sperm suspensions}

\subsubsection{Principle}

In this procedure, a sensitive luminometer is used to measure low amounts of light generated by human spermatozoa in the presence of a chemiluminescent probe, such as luminol. The methodology described employs a mixture of luminol and horseradish peroxidase to make sensitive measurements of hydrogen peroxide generation. Other probes (e.g. lucigenin) can also be used to monitor the production of ROS by washed human ejaculates (Aitken et al., 1992; McKinney et al., 1996).

Signals generated in response to the probe formyl-methionyl-leucyl-phenylalanine (FMLP) are specific for the leukocyte population, since there are no FMLP receptors on the surface of human spermatozoa (Krausz et al., 1992). Responses can be calibrated with suspensions containing known numbers of polymorphonuclear leukocytes (see Fig. 4.1).

Comment 1: The precise activity measured by these probes is still open to question (Aitken et al., 2004) but the data generated reflect the function of the spermatozoa (Zorn et al., 2003; Said et al., 2004).

Comment 2: A single leukocyte can generate at least 100 times more ROS than a spermatozoon. A low level of leukocyte contamination can therefore have a major influence on the chemiluminescent signals generated by a sperm suspension.

\subsubsection{Reagents}

1. Hanks' balanced salt solution (HBSS), without phenol red: see Appendix 4, section A4.5.

2. Krebs-Ringer medium (KRM), without phenol red: see Appendix 4, section A4.7.

3. Luminol, $25 \mathrm{mmol} / \mathrm{l}$ : dissolve $29 \mathrm{mg}$ of luminol (5-amino-2,3-dehydro-1, 4-phthalazinedione) in $10 \mathrm{ml}$ of dimethyl sulfoxide (DMSO).

4. Horseradish peroxidase (HRP) (type $\mathrm{VI}, 310 \mathrm{IU} / \mathrm{mg}$ protein): dissolve $5 \mathrm{mg}$ (1550 IU) in $1 \mathrm{ml}$ of KRM.

5. FMLP (leukocyte-specific probe, $10 \mathrm{mmol} / \mathrm{l}$ ): dissolve $44 \mathrm{mg}$ of $\mathrm{FMLP}$ in $10 \mathrm{ml}$ of DMSO. 
6. Phorbol 12-myristate 13 -acetate (PMA), $1 \mathrm{mmol} / \mathrm{l}$ stock solution: dissolve $6.2 \mathrm{mg}$ of PMA in $10 \mathrm{ml}$ of DMSO. Dilute $1 \mathrm{mmol} / \mathrm{IPMA} 100$-fold in DMSO to give a $10 \mu \mathrm{mol} / /$ working solution.

7. Zymosan.

8. Gelatin: $0.1 \%(1 \mathrm{~g} / \mathrm{l})$ in HBSS.

\subsubsection{Opsonization of zymosan}

1. Suspend $500 \mathrm{mg}$ of zymosan in $10 \mathrm{ml}$ of HBSS.

2. Vortex vigorously.

3. Boil for 20 minutes in a beaker, covered to prevent evaporation.

4. Centrifuge at $500 \mathrm{~g}$ for 5 minutes.

5. Wash the pellet with $10 \mathrm{ml}$ of HBSS.

6. Repeat the wash.

7. Resuspend the pellet in $5 \mathrm{ml}$ of fresh human serum by gentle pipetting.

8. Incubate for 20 minutes.

9. Centrifuge at $500 \mathrm{~g}$ for 5 minutes.

10. Wash the pellet with $10 \mathrm{ml}$ of HBSS.

11. Repeat the wash.

12. Resuspend the pellet to a concentration of $50 \mathrm{mg} / \mathrm{ml}$ in $10 \mathrm{ml}$ of HBSS $+0.1 \%$ $(1 \mathrm{~g} / \mathrm{l})$ gelatin by gentle pipetting.

13. Store at $-20 \stackrel{\circ}{ } \mathrm{C}$ until needed.

\subsubsection{Detecting spontaneous generation of ROS}

1. Mix the semen sample well (see Box 2.3) and remove a volume containing at least $10 \times 10^{6}$ spermatozoa for assessment for ROS.

2. Wash the spermatozoa (see Section 5.3) in KRM and adjust to $10 \times 10^{6}$ spermatozoa per ml.

3. Pipette $400 \mu$ l of the washed spermatozoa suspension suspended in KRM without phenol red into a disposable luminometer container. Take care not to create air bubbles.

4. Add $4 \mu$ l of $25 \mathrm{mmol} / /$ luminol.

5. Add $8 \mu \mathrm{l}$ of horseradish peroxidase $(1550 \mathrm{lU} / \mathrm{ml})$ solution.

6. Monitor the chemiluminescent signal in the luminometer at $37^{\circ} \mathrm{C}$ for about 5 minutes until it has stabilized. 
ROS generation by seminal leukocytes can be stimulated by the addition of FMLP, zymosan or PMA, but PMA also stimulates ROS production by spermatozoa.

\subsubsection{FMLP provocation of ROS generation by leukocytes}

Add $2 \mu \mathrm{l}$ of $10 \mathrm{mmol} / \mathrm{FMLP}$ to the above sample to stimulate a chemiluminescent signal from any leukocytes that are present in the sperm suspension (see Fig. 4.2).

\subsubsection{Zymosan provocation of ROS generation by leukocytes}

Add $20 \mu \mathrm{l}$ of the opsonized zymosan material to the above sample to stimulate a chemiluminescent signal from any leukocytes that are present in the sperm suspension. The size of the signal subsequently generated is directly proportional to the level of leukocyte contamination (see Fig. 4.1).

Fig. 4.1 Chemiluminescence generated in response to opsonized zymosan treatment

A log-linear relationship exists between the leukocyte concentration and the chemiluminescence signal.

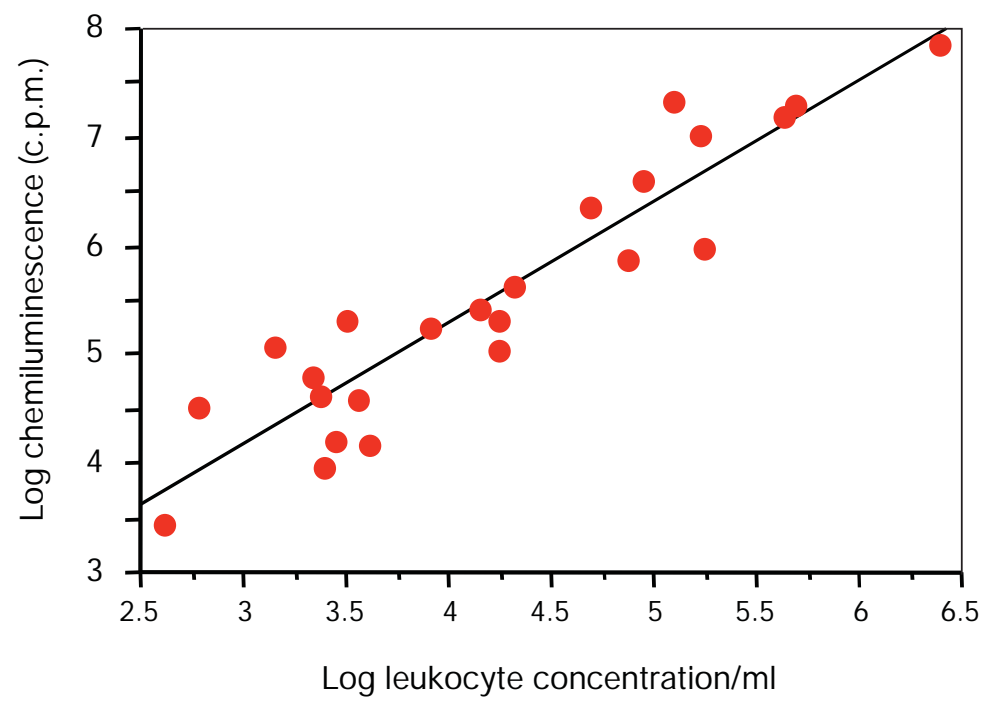

Data courtesy of RJ Aitken.

\subsubsection{PMA provocation of ROS generation by leukocytes and spermatozoa}

1. Dilute the stock PMA solution 100 -fold in DMSO to give a $10 \mu \mathrm{mol} / \mathrm{l}$ working stock solution.

2. Wait for the FMLP or opsonized zymosan signal to subside.

3. Add $4 \mu \mathrm{l}$ of $10 \mu \mathrm{mol} / \mathrm{P} \mathrm{PMA}$ to the same sperm suspension (final concentration $100 \mathrm{nmol} / \mathrm{l}$ ) to stimulate a chemiluminescent signal from spermatozoa (see Fig. 4.2). 
Fig. 4.2 Relative contributions made by leukocyte and sperm subpopulations to the reactive-oxygen-generating capacity of the cell suspension

(a) In the presence of leukocyte contamination, a burst of ROS generation is observed on addition of the leukocyte-specific probe FMLP. The subsequent addition of PMA generates a sustained, intense chemiluminescent signal from both the spermatozoa and leukocyte populations.

(b) In the absence of leukocyte contamination, the FMLP response is lost, while PMA elicits a pronounced chemiluminescent signal from the spermatozoa (see also Krausz et al., 1992).
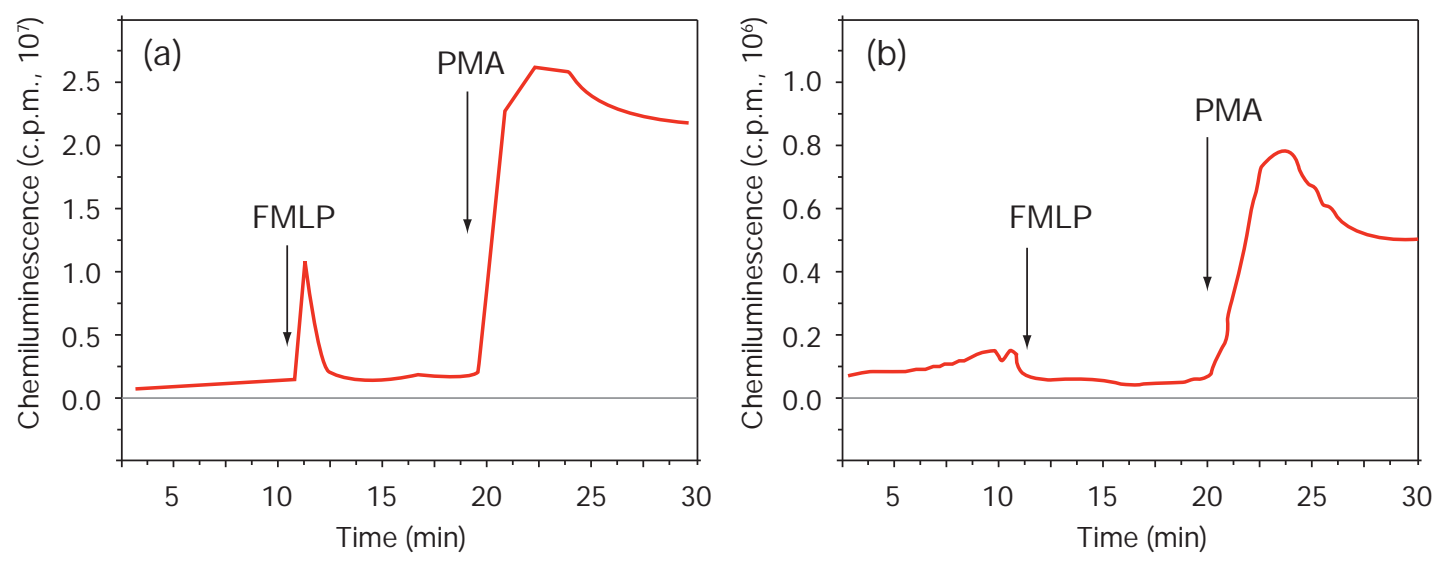

Data courtesy of RJ Aitken.

\subsubsection{Results}

Examine the graphical output after stimulation for evidence of leukocyte contamination.

\subsection{Human sperm-0ocyte interaction tests}

The binding of spermatozoa to the zona pellucida initiates the acrosome reaction, releases free and exposes bound lytic acrosomal components, and allows the spermatozoa to penetrate through the zona matrix, driven by the increased flagellar thrusting of hyperactivated motility. To evaluate the binding events, non-viable, non-fertilizable human oocytes from autopsy, surgic ally removed ovaries or failed in-vitro fertilization may be used. These tests can be performed using oocytes that have been stored in salt, but are usually limited by the lack of availability of human oocytes (Yanagimachi et al., 1979; Kruger et al., 1991; Liu \& Baker, 1992b; Liu et al., 2004).

\subsection{Human zona pellucida binding tests}

One zona pellucida binding assay, the hemizona assay (Burkman et al., 1988), involves microdissection of the zona pellucida into equal halves and the exposure of each matching half to the same concentration of test or control spermatozoa. Another sperm-zona binding assay (Liu et al., 1988, 1989) involves labelling the 
test sample spermatozoa with a fluorescent dye (e.g. fluorescein) and a control sperm sample with another dye (e.g. rhodamine). The number of spermatozoa from the test and control samples bound to the same intact zona are counted and reported as a ratio. Results from both zona binding tests have been shown to be correlated with fertilization rates in vitro (Liu \& Baker, 2003).

It may be clinically useful to evaluate the number of bound spermatozoa in cases of low or failed in-vitro fertilization, idiopathic infertility and teratozoospermia (Franken et al., 1989; Liu \& Baker, 1992a, 2004). The binding of few or no spermatozoa to the zona pellucida usually indicates a sperm defect.

\subsection{Assessment of the acrosome reaction}

The physiological acrosome reaction occurs at the zona pellucida after sperm binding. The zona pellucida-induced acrosome reaction can be assessed on spermatozoa removed from the surface of the zona pellucida or exposed to disaggregated human zona pellucida proteins (Liu \& Baker, 1994, 1996; Franken et al., 2000). In cases of teratozoospermia and oligozoospermia, some men may have otherwise normal semen analyses, but spermatozoa that display disordered zona pellucida-induced acrosome reactions. Others may have spermatozoa that exhibit normal zona pellucida binding but have a poor zona pellucida-induced acrosome reaction (Liu et al., 2004). These tests are limited by the restricted availability of human zonae pellucidae. Zonae from other primates cannot be used as surrogates because of their restricted binding specificity (Bedford, 1977; Liu et al., 1991b; Oehninger et al., 1993). Other stimuli, such as calcium ionophores, will induce the acrosome reaction but the results are not related to those obtained from the zona pellucida-induced acrosome reaction (Liu \& Baker, 1996). Acrosomal status after induction of the acrosome reaction can be assessed by microscopy or flow cytometry (Fenichel et al., 1989; Henley et al., 1994; Cooper \& Yeung, 1998) with fluorescently labelled lectins, such as $\mathrm{P}$ isum sativum (pea agglutinin) (see Section 4.4.1) or A rachis hypogaea (peanut lectin), or monoclonal antibodies against the acrosome antigen CD46 (Cross, 1995).

\subsubsection{Procedure for the fluorescence assessment of acrosomal status}

The method was originally developed by Cross et al. (1986) and subsequently modified by Liu \& Baker (1988). The modified procedure is simpler, reproducible and produces very clear images (Fig. 4.3). It is preferable to use a highly motile sperm preparation free from contaminants such as leukocytes, germ cells and dead spermatozoa. Thus either the sample should be washed (see Section 5.3), or swim-up (see Section 5.4) or density-gradient preparations (see Section 5.5) should be made, depending on the quality of the sample.

\subsubsection{Reagents}

1. Pisum sativum agglutinin (PSA) labelled with fluorescein isothiocyanate (FITC) (PSA-FITC).

2. Phosphate-buffered saline (PBS), $\mathrm{pH} 7.4$. 
3. $\mathrm{NaCl}, 0.9 \%(9 \mathrm{~g} / \mathrm{l})$ : dissolve $0.9 \mathrm{~g}$ of $\mathrm{NaCl}$ in $100 \mathrm{ml}$ of purified water.

4. Ethanol $95 \%(\mathrm{v} / \mathrm{v})$.

5. PSA stock solution: dilute $2 \mathrm{mg}$ of PSA-FITC in $4 \mathrm{ml}$ of PBS. Store $0.5-\mathrm{ml}$ aliquots at $-20{ }^{\circ} \mathrm{C}$.

6. PSA working solution: dilute $0.5 \mathrm{ml}$ of PSA stock solution in $10 \mathrm{ml}$ of PBS and store at $4{ }^{\circ} \mathrm{C}$. This solution is stable for up to 4 weeks.

\subsubsection{Simple washing of spermatozoa}

1. Mix the semen sample well (see Box 2.3) and remove an aliquot of about $0.2 \mathrm{ml}$.

2. Dilute to $10 \mathrm{ml}$ with $0.9 \%(9 \mathrm{~g} / \mathrm{l})$ saline.

3. Centrifuge at $800 \mathrm{~g}$ for 10 minutes.

4. Tip off and discard all but $20-40 \mu$ l of the supernatant.

5. Resuspend the sperm pellet in the remaining supernatant by gentle pipetting.

6. Repeat the washing procedure.

\subsubsection{Treating purified sperm preparations}

1. Dilute swim-up (see Section 5.4) or once-washed density-gradient preparations (see Section 5.5) to $10 \mathrm{ml}$ with saline.

2. Centrifuge at $800 \mathrm{~g}$ for 10 minutes.

3. Tip off and discard all but $20-40 \mu$ of the supernatant.

4. Resuspend the sperm pellet in the remaining supernatant by gentle pipetting.

\subsubsection{Preparing a smear}

1. Prepare replicate sperm smears about $1 \mathrm{~cm}$ long from about $5 \mu$ l of suspension.

2. Inspect the wet smears by phase-contrast microscopy $(\times 400)$.

3. Ensure that the spermatozoa are evenly distributed on the slides without clumping.

4. Allow the slides to air dry.

5. Fix in $95 \%(v / v)$ ethanol for 30 minutes.

6. Allow to air dry.

\subsubsection{Staining with PSA-FITC}

1. Pour $10 \mathrm{ml}$ of PSA-FITC working solution into a vertical staining jar.

2. Immerse the fixed and air-dried slides in the PSA-FITC stain.

3. Allow to stain for more than 1 hour at $4{ }^{\circ} \mathrm{C}$. 
4. Wash each slide with purified water and mount in ethanol-soluble medium (see Sections 2.14.2.4 and 2.14.2.5).

Note: Longer staining times (up to 18 hours) will not affect PSA results. Shorter times (less than 1 hour) will make it difficult to score the slide.

\subsubsection{Scoring}

View the slide with fluorescence optics at $\times 400$ magnification with oil immersion at 450-490 nm excitation. Categorize the spermatozoa as follows.

1. Acrosome-intact (AI): spermatozoa in which more than half the head is brightly and uniformly fluorescing (see Fig 4.3).

2. Acrosome-reacted (AR): spermatozoa with only a fluorescing band at the equatorial segment or no fluorescing stain at all in the acrosome region (see Fig. 4.3).

3. Abnormal acrosomes: all other spermatozoa.

\subsubsection{Counting acrosome-reacted spermatozoa}

1. Tally the number in each acrosomal category (AI and AR) with the aid of a laboratory counter.

2. Evaluate 200 spermatozoa in each replicate, in order to achieve an acceptably low sampling error (see Box 2.5).

3. Calculate the average and difference of the two percentages of acrosomereacted spermatozoa from the replic ate slides.

4. Determine the acceptability of the difference from Table 2.1 or Fig. A7.2, Appendix 7. (Each shows the maximum difference between two percentages that is expected to occur in $95 \%$ of samples because of sampling error alone.)

5. If the difference between the percentages is acceptable, report the average percentage of acrosome-reacted spermatozoa. If the difference is too high, reassess the two slides (see Box 2.6).

6. Report the percentage of acrosome-reacted spermatozoa to the nearest whole number. 
Fig. 4.3 Fluorescent $P$ isum sativum agglutinin (PSA) staining of human spermatozoa

Al spermatozoa, with stained proximal heads (acrosome), and AR spermatozoa, with stained equatorial bands or post-acrosomal regions, are shown.

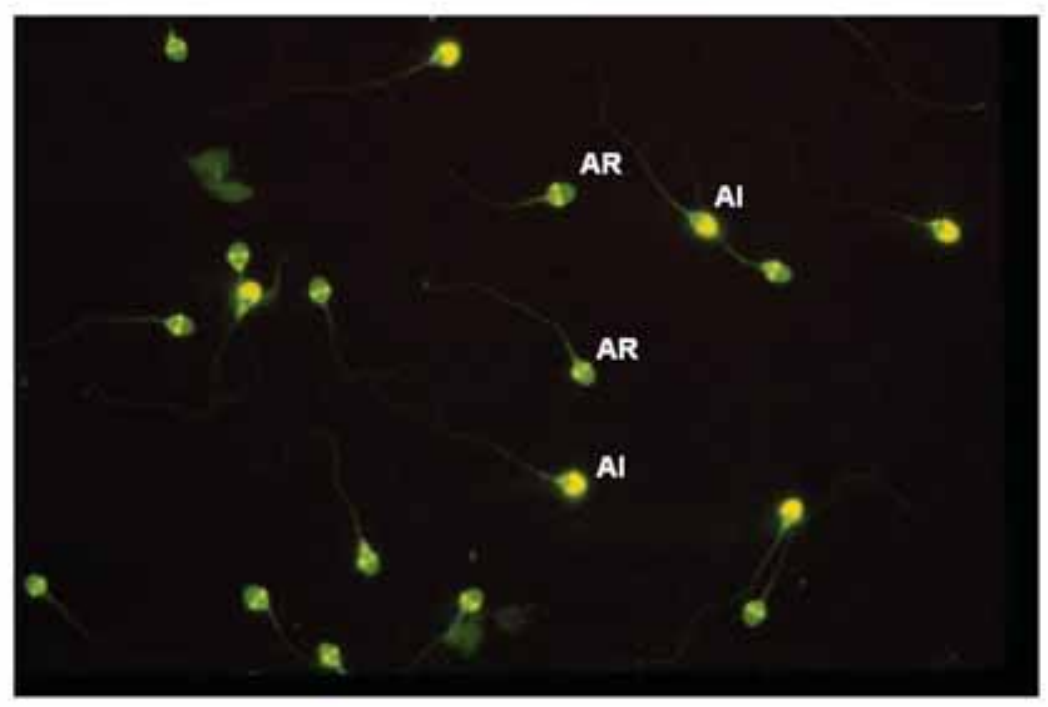

Micrograph courtesy of HWG Baker.

\subsubsection{Induced acrosome reaction assay}

The acrosome reaction is an exocytotic process that occurs after spermatozoa bind to the zona pellucida and must take place before the spermatozoon can penetrate the oocyte vestments and fuse with the oocyte. Calcium influx is believed to be an initiating event in the normal acrosome reaction. Inducing calcium influx by using a calcium ionophore is one way of testing the competence of capacitated spermatozoa to undergo the acrosome reaction (Aitken et al., 1993). This is the basis of this assay, also called the acrosome reaction after ionophore challenge (ARIC) test. However, further evaluation is needed before testing of acrosome status can be considered a routine clinical assay.

\subsubsection{Reagents}

1. Ham's F-10 medium (see Appendix 4, section A4.4) containing 3.5\% (35 g/l) human serum albumin (HSA).

2. Biggers, Whitten and Whittingham (BWW) stock solution: see Appendix 4, section A4.1.

3. Dimethyl sulfoxide (DMSO).

4. Ionophore $A 23187,1 \mathrm{mmol} / \mathrm{l}$ stock solution: dissolve $5.23 \mathrm{mg}$ of $\mathrm{A} 23187$ in $10 \mathrm{ml}$ of DMSO.

5. Glutaraldehyde $3 \%(\mathrm{v} / \mathrm{v})$, or ethanol $70 \%(\mathrm{v} / \mathrm{v})$. 


\subsubsection{Procedure}

1. Allow 30-60 minutes for complete liquefaction of the fresh semen.

2. Prepare the Ham's F-10-HSA capacitation-inducing medium fresh for each assay.

3. Warm the medium to $37{ }^{\circ} \mathrm{C}$ before use, preferably in a $5 \%(v / v) \mathrm{CO}_{2}$-in-air incubator.

4. Prepare a highly motile sperm population, free from contaminants such as leukocytes, germ cells and dead spermatozoa, by density-gradient centrifugation (see Section 5.5) using fresh Ham's F-10 HSA medium.

5. Prepare control and replic ate experimental tubes, each containing approximately $1 \mathrm{ml}$ of suspension with $1 \times 10^{6}$ motile spermatozoa.

6. Incubate the sperm suspensions for 3 hours at $37{ }^{\circ} \mathrm{C}$ in an atmosphere of $5 \%$ (v/v) $\mathrm{CO}_{2}$ in air to induce capacitation (loosen the cap of the tube to allow gas exchange). If a $\mathrm{CO}_{2}$ incubator is not available, use a Hepes-buffered medium (see Appendix 4, section A4.1, Note 1), cap the tubes tightly and incubate at $37{ }^{\circ} \mathrm{C}$.

7. Add $10 \mu \mathrm{l}$ of $\mathrm{A} 23187$ stock solution $(1 \mathrm{mmol} / \mathrm{l})$ to the replic ate experimental tubes to yield a final concentration of $10 \mu \mathrm{mol} / \mathrm{l}$.

8. Add $10 \mu$ l of DMSO to the control tube.

9. Incubate all the tubes at $37^{\circ} \mathrm{C}$ for 15 minutes.

10. Remove a small aliquot from each tube for motility determination.

11. Stop the reaction by adding $100 \mu$ of $3 \%(\mathrm{v} / \mathrm{v})$ glutaraldehyde or $70 \%(\mathrm{v} / \mathrm{v})$ ethanol.

12. Transfer the fixed spermatozoa to precleaned microscope slides and dry in air.

13. Stain the spermatozoa using fluorescent labels (see Section 4.4.1.5).

14. Evaluate by fluorescence microscopy at $\times 400$ magnification with oil immersion at $450-490 \mathrm{~nm}$ excitation.

15. Assess the percentage of acrosome-reacted spermatozoa in the experimental samples (test \% AR) and the control samples (control \% AR).

\subsubsection{Scoring}

1. The acrosome reaction after ionophore challenge (ARIC) is the test \%AR minus the control \% AR.

2. The normal difference is approximately $15 \%$ AR.

3. Values under $10 \%$ AR are considered abnormal.

4. Values between $10 \%$ AR and $15 \%$ AR suggest that sperm function may be abnormal.

5. Control values above $15 \%$ indicate a spontaneous and premature AR. 


\subsubsection{Quality control}

1. A positive control sample (semen from a man whose spermatozoa have previously responded well to ionophore ( $>15 \%$ AR $)$ ) should be run each time the test is performed.

2. Each time a new batch of stain is prepared, perform a cross-over test with the old stain, using positive-control spermatozoa with a known response, to ensure that the stain has been made properly.

\subsection{Zona-free hamster oocyte penetration test}

The fusion of human spermatozoa to the hamster oocyte is functionally the same as that with the human vitelline membrane, since it is initiated by the plasma membrane overlying the equatorial segment of acrosome-reacted human spermatozoa. The hamster oocyte penetration (HOP) test, or sperm penetration assay, differs from the physiological situation in that the zona pellucida is absent. A standard protocol for this test is given below.

Comment: The conventional hamster oocyte test depends on the occurrence of spontaneous acrosome reactions in populations of spermatozoa incubated for prolonged periods in vitro. Since this procedure is less efficient than the biological process and may involve different mechanisms, false-negative results (men whose spermatozoa fail in the hamster oocyte test but successfully fertilize human oocytes in vitro or in vivo) have frequently been recorded (WHO, 1986). Despite this potentially confounding limitation, the test provides information on the fusinogenic nature of capacitated sperm head membranes.

Two of the key intracellular signals that initiate the acrosome reaction following sperm-zona pellucida interaction are an influx of calcium and cytoplasmic alkalinization. As both can be generated artificially with a divalent cation ionophore (Aitken et al., 1993), an alternative method using ionophore-stimulated spermatozoa is also described.

\subsubsection{Protocol}

\subsubsection{Reagents}

1. BWW stock solution: see Appendix 4, section A4.1.

2. Hyaluronidase (300-500 IU/mg).

3. Trypsin type I (10 000 BAEE U/mg).

4. Wax (melting point $48-66^{\circ} \mathrm{C}$ ).

5. Petroleum jelly.

6. Mineral oil.

7. Zona-free hamster oocytes: these can be purchased commercially or obtained by superovulation of hamsters (see Box 4.1). 
8. Dimethyl sulfoxide (DMSO).

9. Ionophore (for alternative protocol) $1 \mathrm{mmol} / \mathrm{l}$ stock solution: dissolve $5.23 \mathrm{mg}$ of the divalent cation ionophore A23187 in $10 \mathrm{ml}$ DMSO.

4.5.1.2 Standard protocol not incorporating ionophore challenge

1. Mix the semen sample well (see Box 2.3).

2. Prepare semen samples by density-gradient centrifugation (see Section 5.5) or swim-up (see Section 5.4).

3. Remove most of the supernatant from the pellet.

4. Dislodge the pellet by gentle pipetting and establish the concentration of spermatozoa in the pellet (see Sections 2.7 and 2.8).

5. Dilute the pellet to approximately $10 \times 10^{6}$ spermatozoa per $\mathrm{ml}$ in approximately $0.5 \mathrm{ml}$ of medium.

6. Incline the tube at an angle of $45^{\circ}$ to the horizontal to increase the surface area.

7. Incubate the sperm suspensions for $18-24$ hours at $37{ }^{\circ} \mathrm{C}$ in an atmosphere of $5 \%(\mathrm{v} / \mathrm{v}) \mathrm{CO}_{2}$ in air to induce capacitation (loosen the cap of the tube to allow gas exchange). If a $\mathrm{CO}_{2}$ incubator is not available, use a Hepes-buffered medium (see Appendix 4, section A4.1, Note 1), cap the tubes tightly and incubate at $37^{\circ} \mathrm{C}$.

8. Return the tubes to the vertical position for 20 minutes to allow settling of any immotile cells after capacitation.

9. Aspirate motile spermatozoa from the top third of the supernatant, being careful not to disturb the dead spermatozoa at the interface, and transfer them to a new tube.

10. Adjust the concentration to $3.5 \times 10^{6}$ motile spermatozoa per ml of medium.

11. With a positive-displacement pipette, aspirate known volumes $(50-150 \mu \mathrm{l})$ of sperm suspension and slowly dispense them into a small Petri dish. With a plastic disposable pipette, cover the droplet with prewarmed mineral oil equilibrated in $\mathrm{CO}_{2}$, being careful not to disturb the sperm suspension. Add enough oil to surround and just cover each droplet of spermatozoa.

\subsubsection{Alternative protocol incorporating a calcium $\left(\mathrm{Ca}^{2+}\right)$ ionophore}

1. Prepare a highly motile sperm population by density-gradient centrifugation, as described in Section 5.5.

2. Aspirate the pellet at the bottom of the $80 \%$ density-gradient medium fraction and transfer it into $8 \mathrm{ml}$ of BWW.

3. Centrifuge at $500 \mathrm{~g}$ for 5 minutes.

4. Decant most of the supernatant from the pellet and dislodge the pellet by gentle pipetting. 
5. Establish the concentration of spermatozoa in the pellet (see Sections 2.7 and 2.8 ) and dilute to approximately $5 \times 10^{6}$ motile spermatozoa per $\mathrm{ml}$ of fresh BWW.

6. Add 1.25 and $2.5 \mu \mathrm{l}$ of $\mathrm{A} 23187$ stock solution $(1 \mathrm{mmol} / \mathrm{l})$ to separate $1-\mathrm{ml}$ aliquots of sperm suspension, to achieve two final concentrations of 1.25 and $2.5 \mu \mathrm{mol} / \mathrm{l}$, respectively.

7. Incubate the spermatozoa with the ionophore for 3 hours at $37^{\circ} \mathrm{C}$.

8. Centrifuge the cells at $500 \mathrm{~g}$ for 5 minutes.

9. Decant most of the supernatant from the pellet and dislodge the pellet by gentle pipetting.

10. Assess the percentage of motile spermatozoa.

11. Dilute to approximately $3.5 \times 10^{6}$ motile spermatozoa per $\mathrm{ml}$ of fresh BWW. Valid results can still be obtained using concentrations as low as $1 \times 10^{6}$ motile spermatozoa per ml (Aitken \& Elton, 1986).

12. Disperse spermatozoa under mineral oil, as described in 4.5.1.2, step 11.

Note: The dose-response curve for ionophore treatment varies between individuals, so it is preferable to test both ionophore concentrations.

\section{Box 4.1 Induction of ovulation in hamsters}

Ensure that all legal requirements for injecting living animals are satisfied. Prepare solutions of the appropriate dose of pregnant mare's serum gonadotrophin (PMSG) and human chorionic gonadotrophin (hCG). Dispense into small vials. Store at $-20{ }^{\circ} \mathrm{C}$ until use. Inject immature hamsters, or mature hamsters on day 1 of the estrous cycle, intraperitoneally (i.p.) with 30 IU of PMSG. After 48-72 hours, inject them with $40 \mathrm{IU}$ of hCG i.p. Grasp the animal's back and pull the abdominal skin taut over its belly with one hand; with the other deliver the hormone into the abdominal cavity (just above the hip joints) from a 1-ml syringe through a 21-gauge needle. Change needles between animals to ensure easy penetration of the skin and minimal discomfort to the animals.

\subsubsection{Collecting the ovaries}

1. Recover the oocytes within 18 hours after the injection of hCG by sacrificing the animals according to methods approved by the relevant animal care and use committee.

2. Place the hamsters on their back and dampen the abdominal fur with $95 \%(\mathrm{v} / \mathrm{v})$ ethanol.

3. Grasp the skin with toothed forceps and cut through the skin and muscle with scissors to expose the uterus and ovaries.

4. Wipe the forceps and scissors free of fur with $95 \%(v / v)$ ethanol.

5. Push the intestines out of the abdominal cavity to expose the uterine horns. 
6. Grasp one uterine horn with the forceps and lift it out of the abdominal cavity to expose the oviduct, ovary and ovarian ligament.

7. Hold the most distal portion of the uterine horn with the forceps and cut through the tip of the uterus just beneath the forceps. Cut off the ovary and place it in warm $\left(37^{\circ} \mathrm{C}\right) \mathrm{BWW}$ in a small Petri dish.

8. Collect the second ovary in the same way.

\subsubsection{Collecting the cumulus masses}

1. Examine the ovaries by transillumination in a dissecting microscope to locate the cumulus cells containing the oocytes in the swollen portion of the oviduct.

2. Hold the oviduct with forceps and puncture the swollen area with a 21-gauge needle. The cumulus mass will pour out of the puncture hole.

3. Tease out the cumulus mass with the needle. Squeeze the oviduct with the forceps to remove all the cumulus mass.

\subsubsection{Recovering and treating the oocytes}

1. Gather the cumulus cells with needle and forceps and place the cells in a watchglass dish, spot plate or other shallow container containing $0.1 \%(1 \mathrm{~g} / \mathrm{l})$ hyaluronidase $(300-500 \mathrm{IU} / \mathrm{ml})$ in warm, $\mathrm{CO}_{2}$-equilibrated $\mathrm{BWW}$.

2. Incubate the container, covered with aluminium foil to protect the cells from light, for 10 minutes at room temperature. Observe the separation of the cumulus cells in a dissecting microscope.

3. Use a flame-drawn glass pipette (see Box 4.2) to transfer freed oocytes from the hyaluronidase to the warm equilibrated BWW.

4. Rinse the recovered oocytes twice in BWW by transferring them into fresh drops of warm, equilibrated BWW. This can be done in a glass multi-well dish or spot plate. Rinse the pipette with BWW between each oocyte transfer.

5. Treat the oocytes with $0.1 \%(1 \mathrm{~g} / \mathrm{l})$ trypsin $(10000 \mathrm{lU} / \mathrm{ml})$ for approximately 1 minute at room temperature to remove the zonae pellucidae. Observe the digestion of the zona in a dissecting microscope and remove the oocytes as soon as the zona has dissolved.

6. Wash the oocytes three times more with BWW.

7. Warm the isolated oocytes to $37^{\circ} \mathrm{C}$ and introduce them into the sperm suspensions. Alternatively, they may be stored at $4{ }^{\circ} \mathrm{C}$ for up to 24 hours before use.

\section{B ox 4.2 Preparation of glass pipettes}

Rotate a glass capillary tube or Pasteur pipette just above a Bunsen burner flame, holding the ends of the glass tube with both hands and rolling it back and forth over the flame to ensure even heating of the glass. J ust as the glass starts to melt, pull your hands apart quickly to stretch it. Snap off the thread-like strand of glass to the desired width (approximately $1 \mathrm{~mm}$ ) of the pipette opening. Attach the non-drawnout end of the pipette to a 1-ml syringe with tubing. 


\subsubsection{Co-incubation of gametes}

1. Dispense the zona-free hamster oocytes into several droplets, with about five oocytes per drop (i.e. for 20 oocytes per semen sample prepare four aliquots of five oocytes per drop).

2. Load groups of about five oocytes into the glass pipette with little medium so as not to dilute the sperm suspensions too much.

3. Insert the pipette tip directly into the centre of one droplet of sperm suspension and slowly dispense the oocytes. Maintain positive pressure to prevent the mineral oil from entering the pipette and take care not to introduce air bubbles into the sperm suspension.

4. Wipe any excess oil from the pipette tip after removal from the sperm suspension.

5. Repeat step 3 until all oocytes have been transferred to the sperm suspensions.

6. Rinse the pipette thoroughly in BWW after each egg transfer to prevent crosscontamination of spermatozoa.

7. Incubate the gametes for 3 hours at $37{ }^{\circ} \mathrm{C}$ in an atmosphere of $5 \%(v / v) \mathrm{CO}_{2}$ in air.

8. Recover the oocytes from the oil droplets. Take care to wipe any oil from the tip of the pipette before transferring the oocytes to BWW.

9. Wash the oocytes free of loosely adherent spermatozoa with the flame-drawn Pasteur pipette, by rinsing in BWW.

\subsubsection{Analysing the oocytes}

1. Place four pillars of wax-petroleum jelly mixture (see Box 3.1) in a rectangular pattern to support the coverslip $(22 \mathrm{~mm} \times 22 \mathrm{~mm}$, thickness number 1.5 , $0.17 \mathrm{~mm}$ ) at its corners.

2. Place a small droplet of oocyte-containing BWW in the centre of the four pillars.

3. Lower the coverslip over the wax pillars and gently press it down, to begin to flatten the oocytes. A well-flattened oocyte is required for optimal observation of decondensed sperm heads.

4. If necessary, add more BWW to flood the slide to prevent squashing of the oocytes.

5. Examine the preparation by phase-contrast microscopy at $\times 200$ magnification.

6. Count the number of decondensed sperm heads with an attached or closely associated tail (see Fig. 4.4).

7. Record the percentage of eggs penetrated by at least one spermatozoon and the number of spermatozoa per penetrated egg. 
8. Record the presence of any spermatozoa that remain bound to the surface of the oocyte after the initial washing procedure, since this may give some indication of the proportion of the sperm population that has undergone the acrosome reaction.

Fig. 4.4 Phase-contrast micrograph of a zona-free hamster oocyte containing human spermatozoa

The wide arrows indicate the presence of decondensed sperm heads within the ooplasm; the narrow arrows point to non-penetrated spermatozoa on the egg surface.

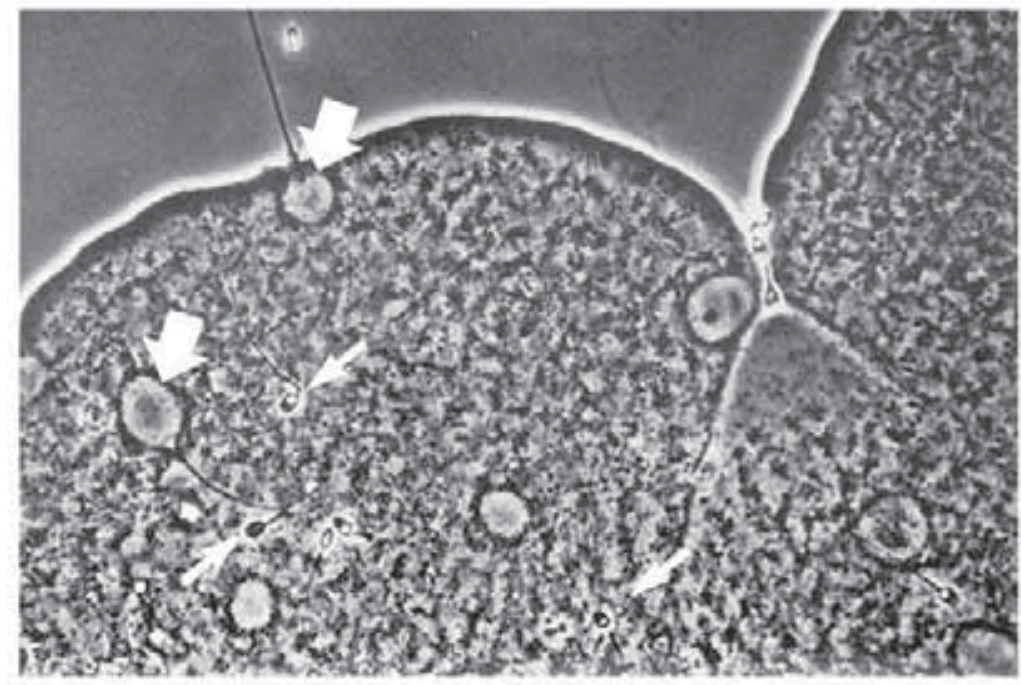

Reproduced from Aitken et al. (1983) with kind permission of Springer Science + Business Media.

\subsubsection{Quality control}

The assays must be performed with a positive control semen sample exhibiting $>50 \%$ penetration.

\subsection{Assessment of sperm chromatin}

Several methods have been used to test the normality of sperm chromatin and DNA. They all use dyes that bind to histone (aniline blue) or nucleic acid (acridine orange, chromomycin) and are assessed histologically or by flow cytometry. Newer methods include those based on assessment of DNA strand breaks, such as terminal deoxynucleotidyl transferase (TdT)-mediated deoxyuridine triphosphate (dUTP)-nick-end labelling (or TUNEL for short (in situ end-labelling, ISEL)), comet assays or sperm chromatin dispersion (SCD). The results of these tests are correlated with each other (Chohan et al., 2006) and with sperm morphology, motility and viability. They may give additional information about fertilization rates with standard IVF and, possibly, spontaneous pregnancy rates. The sperm chromatin structure assay (SCSA) can be predictive of fertilization failure in vivo and in vitro (Evenson \& Wixon, 2006). Whether there is any relationship between the results of these tests and miscarriage or other outcomes of pregnancy is not yet clear. 
PART II.

Sperm preparation 



\section{CHAPTER 5 Sperm preparation techniques}

\subsection{Introduction}

Spermatozoa may need to be separated from seminal plasma for a variety of purposes, such as diagnostic tests of function and therapeutic recovery for insemination and assisted reproductive technologies (ART). If tests of sperm function are to be performed, it is critical that the spermatozoa are separated from the seminal plasma within 1 hour of ejaculation, to limit any damage from products of nonsperm cells.

Comment 1: Counting too few spermatozoa will produce an uncertain result (see Appendix 7, section A7.1.1) which may have consequences for diagnosis and therapy (see Appendix 7, section A7.2). This may be unavoidable when spermatozoa are required for therapeutic purposes and few are available.

Comment 2: When smaller semen volumes are taken and fewer spermatozoa are counted than recommended, the precision of the values obtained will be significantly reduced. When fewer than 400 spermatozoa are counted, report the sampling error for the number of cells counted (see Table 2.2).

5.1.1 When spermatozoa may need to be separated from seminal plasma

Although seminal plasma helps spermatozoa penetrate cervical mucus (Overstreet et al., 1980), some of its components (e.g. prostaglandins, zinc) are obstacles to the achievement of pregnancy when natural barriers are bypassed in ART, such as intrauterine insemination (IUI) or in-vitro fertilization (IVF). The separation of human spermatozoa from seminal plasma to yield a final preparation containing a high percentage of morphologically normal and motile cells, free from debris, non-germ cells and dead spermatozoa, is important for clinical practice. Diluting semen with culture media and centrifuging is still used for preparing normozoospermic specimens for IUI (Boomsma et al., 2004). However, density-gradient centrifugation and direct swim-up are generally preferred for specimens with one or more abnormalities in semen parameters (see e.g. Morshedi et al., 2003). Glass-wool columns are reported to be as effective as density gradients for the separation of spermatozoa from semen with suboptimal characteristics (R hemrev et al., 1989; J ohnson et al., 1996).

\subsubsection{Choice of method}

The choice of sperm preparation technique is dictated by the nature of the semen sample (see Canale et al., 1994). For example, the direct swim-up technique is often used when the semen samples are considered to be largely normal, whereas in cases of severe oligozoospermia, teratozoospermia or asthenozoospermia, density gradients are usually preferred because of the greater total number of motile spermatozoa recovered. Density gradients can also be altered to optimize handling of specific properties of individual samples: the total volume of gradient material can be reduced, limiting the distance that the spermatozoa migrate 
and maximizing total motile sperm recovery, or the centrifugation time can be increased for specimens with high viscosity.

Each laboratory should determine the centrifugal force and centrifugation time necessary to form a manageable sperm pellet. When sperm numbers are extremely low, it may be necessary to modify the centrifugal force or the time, in order to increase the chances of recovering the maximum number of spermatozoa. Modifications to recommended times and centrifugal forces should be rigorously tested prior to clinical implementation. The most suitable method of preparation can be identified from the functional capacity of the prepared spermatozoa, as determined, for example, by the zona-free hamster oocyte penetration test (see Section 4.5).

\subsubsection{Efficiency of sperm separation from seminal plasma and infectious organisms}

The efficiency of a sperm selection technique is usually expressed as the absolute sperm number, the total number of motile spermatozoa, or the recovery of morphologically normal motile spermatozoa. Swim-up generally produces a lower recovery of motile spermatozoa $(<20 \%)$ than does density-gradient centrifugation (>20\% ) (but see $\mathrm{Ng}$ et al., 1992). Swim-up and density-gradient centrifugation also produce different levels of contamination with seminal components in the final sperm preparation. Using the prostatic secretion zinc as a marker of soluble seminal components, Björndahl et al. (2005) demonstrated time-dependent diffusion of zinc from semen into the overlaying swim-up medium. The final zinc concentration in swim-up preparations was greater than that after density-gradient preparation.

Semen samples may contain harmful infectious agents, and technicians should handle them as a biohazard with extreme care. Sperm preparation techniques cannot be considered $100 \%$ effective in removing infectious agents from semen (see Section 5.6). Safety guidelines, as outlined in Appendix 2, should be strictly observed. Good laboratory practice is fundamental to laboratory safety (WHO, 2004).

\subsection{General principles}

Three simple sperm preparation techniques are described in the following sections. For all of them, the culture medium suggested is a balanced salt solution supplemented with protein and containing a buffer appropriate for the environmental conditions in which the spermatozoa will be processed. For assisted reproduction procedures, such as intracytoplasmic sperm injection (ICSI), in-vitro fertilization (IVF), artificial insemination (AI) or gamete intrafallopian transfer (GIFT), it is imperative that the human serum albumin is highly purified and free from viral, bacterial and prion contamination. Albumins specifically designed for such procedures are commercially available. If the incubator contains only atmospheric air and the temperature is $37^{\circ} \mathrm{C}$, the medium should be buffered with Hepes or a similar buffer, and the caps of the tubes should be tightly closed. If the incubator atmosphere is $5 \%(\mathrm{v} / \mathrm{v}) \mathrm{CO}_{2}$ in air and the temperature is $37{ }^{\circ} \mathrm{C}$, then the medium is best buffered with sodium bicarbonate or a similar buffer, and the caps of the test-tubes should be loose to allow gas exchange. Adherence to this will ensure that the culture $\mathrm{pH}$ is compatible with sperm survival. The final disposition of the 
processed spermatozoa will determine which buffered medium is appropriate. For example, sperm function assays in general will require a medium that supports sperm capacitation, and typically contains sodium bicarbonate $(25 \mathrm{mmol} / \mathrm{l})$.

Semen should be collected in a sterile manner (see Section 2.2.3). Sterile techniques and materials are essential when applying a sperm preparation technique for therapeutic applications.

\subsection{Simple washing}

This simple washing procedure provides the highest yield of spermatozoa and is adequate if semen samples are of good quality. It is often used for preparing spermatozoa for IUI.

\subsubsection{Reagents}

1. BWW, Earle's, Ham's F-10 or human tubal fluid (HTF) (commercially available or see Appendix 4, sections A4.1, A4.3, A4.4 and A4.6) supplemented preferably with human serum albumin (HSA), or serum, as described below.

2. HSA, highly purified and free from viral, bacterial and prion contamination and endotoxins.

3. HSA supplement: to $50 \mathrm{ml}$ of medium add $300 \mathrm{mg}$ of HSA, $1.5 \mathrm{mg}$ of sodium pyruvate, $0.18 \mathrm{ml}$ of sodium lactate $(60 \%(\mathrm{v} / \mathrm{v})$ syrup) and $100 \mathrm{mg}$ of sodium bicarbonate.

4. Serum supplement: to $46 \mathrm{ml}$ of medium add $4 \mathrm{ml}$ of heat-inactivated $\left(56{ }^{\circ} \mathrm{C}\right.$ for 20 minutes) client's serum, $1.5 \mathrm{mg}$ of sodium pyruvate, $0.18 \mathrm{ml}$ of sodium lactate $(60 \%(\mathrm{v} / \mathrm{v})$ syrup) and $100 \mathrm{mg}$ of sodium bicarbonate.

\subsubsection{Procedure}

1. Mix the semen sample well (see Box 2.3).

2. Dilute the entire semen sample $1+1(1: 2)$ with supplemented medium to promote removal of seminal plasma.

3. Transfer the diluted suspension into multiple centrifuge tubes, with preferably not more than $3 \mathrm{ml}$ per tube.

4. Centrifuge at 300-500g for 5-10 minutes.

5. Carefully aspirate and discard the supernatants.

6. Resuspend the combined sperm pellets in $1 \mathrm{ml}$ of supplemented medium by gentle pipetting.

7. Centrifuge again at 300-500g for 3-5 minutes.

8. Carefully aspirate and discard the supernatant.

9. Resuspend the sperm pellet, by gentle pipetting, in a volume of supplemented medium appropriate for final disposition, e.g. insemination, so that concentration and motility can be determined (see Sections 2.5 and 2.7). 
Note: The number of washings to remove seminal plasma can be reduced by using fewer tubes and increasing the volume in each tube. If this is done, the centrifugal force and duration of centrifugation should be increased, to ensure complete pelleting of spermatozoa, e.g. 500-600g for 8-10 minutes.

\subsection{Direct swim-up}

Spermatozoa may be selected by their ability to swim out of seminal plasma and into culture medium. This is known as the "swim-up" technique. The semen should preferably not be diluted and centrifuged prior to swim-up, because this can result in peroxidative damage to the sperm membranes (Aitken $\&$ Clarkson, 1988). Thus, a direct swim- up of spermatozoa from semen is the preferred method for separating out motile spermatozoa (see e.g. Mortimer, 1994a, b). The direct swim-up technique can be performed either by layering culture medium over the liquefied semen or by layering liquefied semen under the culture medium. Motile spermatozoa then swim into the culture medium. This procedure gives a lower yield of spermatozoa than washing, but selects them for their motility and is useful where the percentage of motile spermatozoa in semen is low, e.g. for IVF and ICSI.

\subsubsection{Reagents}

1. BWW, Earle's, Ham's F-10 or HTF (Appendix 4, sections A4.1, A4.3, A4.4 and A4.6) supplemented preferably with HSA, or serum, as described below.

2. HSA, highly purified and free from viral, bacterial and prion contamination and endotoxins.

3. HSA supplement: to $50 \mathrm{ml}$ of medium add $300 \mathrm{mg}$ of HSA, $1.5 \mathrm{mg}$ of sodium pyruvate, $0.18 \mathrm{ml}$ of sodium lactate $(60 \%(\mathrm{v} / \mathrm{v})$ syrup) and $100 \mathrm{mg}$ of sodium bicarbonate.

4. Serum supplement: to $46 \mathrm{ml}$ of medium add $4 \mathrm{ml}$ of heat-inactivated $\left(56^{\circ} \mathrm{C}\right.$ for 20 minutes) client's serum, $1.5 \mathrm{mg}$ of sodium pyruvate, $0.18 \mathrm{ml}$ of sodium lactate $(60 \%$ (v/v) syrup) and $100 \mathrm{mg}$ of sodium bicarbonate.

\subsubsection{Procedure}

1. Mix the semen sample well (see Box 2.3).

2. Place $1 \mathrm{ml}$ of semen in a sterile $15-\mathrm{ml}$ conical centrifuge tube, and gently layer $1.2 \mathrm{ml}$ of supplemented medium over it. Alternatively, pipette the semen carefully under the supplemented culture medium.

3. Incline the tube at an angle of about $45^{\circ}$, to increase the surface area of the semen-culture medium interface, and incubate for 1 hour at $37^{\circ} \mathrm{C}$.

4. Gently return the tube to the upright position and remove the uppermost $1 \mathrm{ml}$ of medium. This will contain highly motile sperm cells.

5. Dilute this with $1.5-2.0 \mathrm{ml}$ of supplemented medium. 
6. Centrifuge at $300-500 \mathrm{~g}$ for 5 minutes and discard the supernatant.

7. Resuspend the sperm pellet in $0.5 \mathrm{ml}$ of supplemented medium for assessment of sperm concentration, total motility and progressive motility (see Sections 2.5 and 2.7).

8. The specimen may be used directly for therapeutic or research purposes.

\subsection{Discontinuous density gradients}

Discontinuous density gradients can provide the best selection of good-quality spermatozoa, giving good separation from other cell types and debris. It is easier to standardize than the swim-up technique, and thus results are more consistent. This technique is used to recover and prepare spermatozoa for use in IVF and ICSI.

This method uses centrifugation of seminal plasma over density gradients consisting of colloidal silica coated with silane, which separates cells by their density. In addition, motile spermatozoa swim actively through the gradient material to form a soft pellet at the bottom of the tube. A simple two-step discontinuous density-gradient preparation method is most widely applied, typically with a $40 \%$ (v/v) density top layer and an $80 \%$ (v/v) density lower layer. Sperm preparation using densitygradient centrifugation usually results in a fraction of highly motile spermatozoa, free from debris, contaminating leukocytes, non-germ cells and degenerating germ cells.

A number of commercial products are available for making density gradients suitable for semen processing. These products should be used according to the manufacturers' recommendations. Any departure from procedural recommendations should be evidence-based. Most density-gradient media contain high relative molecular mass components that have inherently low osmolality, so they are usually prepared in medium that is iso-osmotic with female reproductive tract fluids.

\subsubsection{Reagents}

1. BWW, Earle's, Ham's F-10 or HTF (see Appendix 4, sections A4.1, A4.3, A4.4 and A4.6), supplemented preferably with HSA, or serum, as described below.

2. HSA, highly purified and free from viral, bacterial and prion contamination and endotoxins.

3. HSA supplement: to $50 \mathrm{ml}$ of medium add $300 \mathrm{mg}$ of HSA, $1.5 \mathrm{mg}$ of sodium pyruvate, $0.18 \mathrm{ml}$ of sodium lactate $(60 \%(\mathrm{v} / \mathrm{v})$ syrup) and $100 \mathrm{mg}$ of sodium bicarbonate.

4. Serum supplement: to $46 \mathrm{ml}$ of medium add $4 \mathrm{ml}$ of heat-inactivated $\left(56{ }^{\circ} \mathrm{C}\right.$ for $30-45$ minutes) patient's serum, $1.5 \mathrm{mg}$ of sodium pyruvate, $0.18 \mathrm{ml}$ of sodium lactate $(60 \%$ (v/v) syrup) and $100 \mathrm{mg}$ of sodium bic arbonate.

5. Isotonic density-gradient medium: to $10 \mathrm{ml}$ of $10 \times$ concentrated culture medium (commercially available or see Appendix 4, sections A4.1, A4.3, A4.4 and A4.6), add $90 \mathrm{ml}$ of density-gradient medium, $300 \mathrm{mg}$ of HSA, $3 \mathrm{mg}$ of sodium pyruvate, $0.37 \mathrm{ml}$ of sodium lactate $(60 \%(\mathrm{v} / \mathrm{v})$ syrup) and $200 \mathrm{mg}$ of sodium bicarbonate. 
6. Gradient $80 \%(\mathrm{v} / \mathrm{v})$ : to $40 \mathrm{ml}$ of isotonic gradient medium add $10 \mathrm{ml}$ of supplemented medium.

7. Gradient $40 \%(\mathrm{v} / \mathrm{v})$ : to $20 \mathrm{ml}$ of isotonic gradient medium add $30 \mathrm{ml}$ of supplemented medium.

Note: Although these isotonic density-gradient media are often referred to as $100 \%, 80 \%$ and $40 \%(\mathrm{v} / \mathrm{v})$, they are really $90 \%, 72 \%$ and $36 \%(\mathrm{v} / \mathrm{v})$.

\subsubsection{Procedure}

1. Prepare the density-gradient medium in a test-tube by layering $1 \mathrm{ml}$ of $40 \%$ $(\mathrm{v} / \mathrm{v})$ density-gradient medium over $1 \mathrm{ml}$ of $80 \%(\mathrm{v} / \mathrm{v})$ density-gradient medium.

2. Mix the semen sample well (see Box 2.3).

3. Place $1 \mathrm{ml}$ of semen above the density-gradient media and centrifuge at 300-400g for 15-30 minutes. More than one tube per semen sample may be used, if necessary.

4. Remove most of the supernatant from the sperm pellet.

5. Resuspend the sperm pellet in $5 \mathrm{ml}$ of supplemented medium by gentle pipetting (to aid removal of contaminating density-gradient medium) and centrifuge at $200 \mathrm{~g}$ for $4-10$ minutes.

6. Repeat the washing procedure (steps 4 and 5 above).

7. Resuspend the final pellet in supplemented medium by gentle pipetting so that concentration and motility can be determined (see Sections 2.5 and 2.7).

\subsection{Preparing HIV-infected semen samples}

If the human immunodeficiency virus (HIV) is present in semen, viral RNA and proviral DNA can be found free in seminal plasma and in non-sperm cells. As HIV receptors (CD4, CCR5, CXCR4) are expressed only by non-sperm cells, a combination of density-gradient centrifugation followed by swim-up has been proposed as a way of preventing infection of uninfected female partners (Gilling-S mith et al., 2006; Savasi et al., 2007). These procedures were developed to separate virusinfected non-sperm cells and seminal plasma (in the density-gradient supernatant) from HIV-free, motile spermatozoa in the swim-up (from the density-gradient pellet). Prepared samples should be tested by reverse transcription polymerase chain reaction (RT-PCR) before use, and only HIV-free samples used for ART. While results so far are encouraging, there is as yet insufficient evidence of the elimination of risk of HIV infection through sperm preparation.

Note: This technique should be used only in secure facilities to minimize the risk of cross-contamination of HIV-free samples (Gilling-Smith et al., 2005). 


\subsection{Preparing testicular and epididymal spermatozoa}

Spermatozoa recovered from testicular tissue and the epididymis require special preparation.

The typical indication for epididymal aspiration is obstructive azoospermia rather than testicular dysfunction. Consequently, relatively large numbers of spermatozoa can be harvested for therapeutic purposes. Epididymal aspirates can often be obtained with minimal contamination from red blood cells and non-germ cells, making the isolation and selection of motile epididymal spermatozoa relatively straightforward. If large numbers of epididymal spermatozoa are obtained, density-gradient centrifugation is an effective method of preparing them for subsequent use (see Section 5.5). If sperm numbers are low, a simple wash can be performed (see Section 5.3).

Testicular spermatozoa can be retrieved by open biopsy (with or without microdissection) or by percutaneous needle biopsy. Testicular specimens are invariably contaminated with non-germ cells and large numbers of red blood cells, so additional steps are needed to isolate a clean preparation of spermatozoa. In order to free the seminiferous tubule-bound elongated spermatids ("testicular spermatozoa"), enzymatic or mechanical methods are needed. Testicular spermatozoa are prepared for ICSI, since sperm numbers are low and their motility is poor.

5.7.1 Enzymatic method

1. Incubate the testicular tissue with collagenase (e.g. $0.8 \mathrm{mg}$ of Clostridium histolyticum, type $1 \mathrm{~A}$ per $\mathrm{ml}$ of medium) for $1.5-2$ hours at $37^{\circ} \mathrm{C}$, vortexing every 30 minutes.

2. Centrifuge at $100 \mathrm{~g}$ for 10 minutes and examine the pellet.

5.7.2 Mechanical method

1. Macerate the testicular tissue in culture medium with glass coverslips until a fine slurry of dissociated tissue is produced.

2. Alternatively, strip the cells from the seminiferous tubules using fine needles (attached to disposable tuberculin syringes) bent parallel to the base of the culture dish.

5.7.3 Processing sperm suspensions for intracytoplasmic sperm injection

1. Wash the specimens obtained by adding $1.5 \mathrm{ml}$ of culture medium.

2. Centrifuge at $300 \mathrm{~g}$ for $8-10$ minutes.

3. Remove the supernatant and resuspend the pellet in $0.5 \mathrm{ml}$ of fresh culture medium.

4. Estimate the motility and number of spermatozoa in the pellet. (Some specimens with a low number of spermatozoa may need to be resuspended in a lower volume of medium.) 
5. Place a $5-10 \mu l$ droplet of culture medium in a culture dish.

6. Cover it with mineral oil (pre-equilibrated with $\mathrm{CO}_{2}$ ).

7. Introduce $5-10 \mu l$ of the sperm suspension into the culture medium.

8. Carefully aspirate the motile spermatozoa found at the interface between the culture medium and oil with an ICSI pipette.

9. Transfer them to a droplet of viscous solution, e.g. polyvinylpyrrolidone (7-10\% $(100 \mathrm{~g} / \mathrm{l})$ in medium).

\subsection{Preparing retrograde ejaculation samples}

In some men, semen passes into the bladder at ejaculation, resulting in aspermia, or no apparent ejaculate. Confirmation of this situation is obtained by examining a sample of post-ejaculatory urine for the presence of spermatozoa. If pharmacological treatment is not possible or not successful, spermatozoa may be retrieved from the urine. Alkalinization of the urine by ingestion of sodium bicarbonate, for example, will increase the chance that any spermatozoa passing into the urine will retain their motility characteristics (Mahadevan et al., 1981).

At the laboratory, the man should be asked to:

- urinate without completely emptying the bladder;

- produce an ejaculate by masturbation into a specimen container;

- urinate again into a second specimen vessel containing culture medium (to alkalinize the urine further).

Both the ejaculate, if any, and urine samples should be analysed. Because a large volume of urine may be produced, it is often necessary to concentrate the specimen by centrifugation ( $500 \mathrm{~g}$ for 8 minutes) The retrograde specimen, once concentrated, and the antegrade specimen, if produced, can be most effectively processed using the density-gradient preparation method (see Section 5.5).

\subsection{Preparing assisted ejaculation samples}

Semen from men with disturbed ejaculation, or who cannot ejaculate, may be collected by direct vibratory stimulation of the penis or rectal electrical stimulation of the accessory organs. Ejaculates from men with spinal cord injury will frequently have high sperm concentrations, decreased sperm motility and red and white blood cell contamination. Specimens obtained by electro-ejaculation can be processed most effectively by density-gradient centrifugation (see Section 5.5). Regardless of the method of preparation, these types of ejaculates will often contain a high percentage of immotile sperm cells. 


\section{CHAPTER 6 Cryopreservation of spermatozoa}

\subsection{Introduction}

Cryopreservation of spermatozoa is an important part of the work of many semen analysis laboratories, particularly those associated with infertility clinics.

The history of human sperm cryobiology dates from the late 1940s. The discovery that glycerol protected spermatozoa against damage from freezing led to the use of human spermatozoa stored on dry ice at $-79{ }^{\circ} \mathrm{C}$ (Polge et al., 1949; Bunge \& Sherman, 1953; Bunge et al., 1954). Subsequently, liquid nitrogen was used and semen cryopreservation developed rapidly in many countries with the establishment of commercial sperm banks or coordinated national services (Perloff et al., 1964; David et al., 1980; Clarke et al., 1997; Leibo et al., 2002).

A variety of cryopreservation protocols are now used with different cryoprotectants and freezing procedures. Cell survival after freezing and thawing depends largely on minimization of intracellular ice crystal formation. This is done by using appropriate cryoprotectants and applying rates of cooling and warming that minimize the amount of intracellular water subject to ice formation (Sherman, 1990; Keel \& Webster, 1993; Watson, 1995). If the spermatozoa spend significant periods of time above $-130{ }^{\circ} \mathrm{C}$ (the glassy transition temperature), particularly during the thawing process, recrystallization can occur, with growth of potentially damaging intracellular ice crystals.

Human spermatozoa tolerate a range of cooling and warming rates. They are not very sensitive to damage caused by rapid initial cooling (cold shock), possibly because of high membrane fluidity from the unsaturated fatty acids in the lipid bilayer (Clarke et al., 2003). They may also be more resistant than other cells to cryopreservation damage because of their low water content (about 50\%). However, cryopreservation does have an adverse effect on human sperm function, particularly motility. On average, only about $50 \%$ of the motile spermatozoa survive freezing and thawing (Keel \& Webster, 1993). Optimizing the cryopreservation process will minimize this damage and may increase pregnancy rates (Woods et al., 2004).

Pregnancy rates after artificial insemination with cryopreserved donor semen are often related to sperm quality after thawing, timing of insemination and, particularly, recipient factors such as age, previous pregnancy with donor insemination, and ovulatory and uterine tubal disorders (Le Lannou \& Lansac, 1993). If semen is stored under appropriate conditions, there is no obvious deterioration in sperm quality with time; children have been born following fertilization using semen stored for over 28 years (Feldschuh et al., 2005; Clarke et al., 2006).

Spermatozoa may be stored for a variety of reasons (see Box 6.1). In some cases, the cryopreservation procedure may need to be modified (see Section 6.2.2). 


\section{Box 6.1 Reasons for cryopreservation of spermatozoa}

\section{Donor semen}

Semen from healthy donors known or presumed to be fertile may be stored for future use. These donors may be recruited by a clinic or sperm bank and their spermatozoa used anonymously. Alternatively, the recipients may know the donors. Donor spermatozoa can be used for AI, IUI, IVF or ICSI:

- for the partner of an infertile man with no live spermatozoa or elongated spermatids suitable for ICSI, or where treatment has failed or is too costly;

- to prevent transmission of an inherited disorder;

- to prevent fetal haemolytic anaemia from blood group incompatibility;

- after recurrent miscarriage, where donor insemination may result in a successful pregnancy;

- for women who wish to conceive, but do not have a male partner.

Local and national legislation regarding genetic and infection screening should always be complied with.

\section{Fertility preservation}

Semen may be obtained and stored before a man undergoes a procedure or exposure that might prevent or impair his fertility, such as:

- vasectomy (in case of a future change in marital situation or desire for more children);

- treatment with cytotoxic agents or radiotherapy, which is likely to impair spermatogenesis permanently (Meseguer et al., 2006; Schmidt et al., 2004);

- active duty in a dangerous occupation, e.g. in military forces, in countries where posthumous procreation is acceptable.

\section{Infertility treatment}

Spermatozoa may be stored for treatment of the man's partner by artificial insemination by husband's semen (AIH), IUI, IVF or ICSI, in cases of:

- severe oligozoospermia or intermittent presence of motile spermatozoa in the semen (as backup for ICSI) (Bourne et al., 1995);

- treatment of infertility that may not persist, such as surgery for genital tract obstruction or gonadotrophin treatment for hypothalamo-pituitary hypogonadism;

- the need for special collection, such as assisted ejaculation for patients with spinal cord injury, spermatozoa from retrograde ejaculation in urine, or surgical collection from the genital tract;

- men who are unable to provide fresh semen on the day of an ART procedure.

\section{Minimizing infectious disease transmission}

For men with HIV controlled by antiretroviral therapy, samples with an undetectable viral load may be stored for IUI, IVF or ICSI, to attempt conception while reducing the risk of transmission of HIV to the female partner.

Note 1: For fertility preservation or infertility treatment, enough normal specimens should be stored for 10 or more inseminations, to ensure a good chance of pregnancy. With abnormal semen, pooling of multiple samples for AlH has not been proven to be useful. 
Note 2: As only a single spermatozoon is needed for ICSI of each oocyte, cryopreservation of any live spermatozoa is worthwhile.

Note 3: Storage of semen collected before a potentially sterilizing procedure often has significant psychological value, because it gives the hope of future paternity. For men about to undergo therapy with alkylating agents or radiotherapy, the semen must be collected before the therapy starts, because of the risk of mutagenesis in the spermatozoa. All males requiring chemo- or radiotherapy, including adolescents (Kamischke et al., 2004), should be offered the possibility of storage of spermatozoa.

The cryopreservation and subsequent storage of human spermatozoa is a highly complex process that places a special responsibility and potential liability on the laboratory staff. A comprehensive risk assessment is recommended (see Box 6.2).

\section{B ox 6.2 Risk assessment of cryopreservation and storage of human semen}

In assessing the risks associated with cryopreservation and storage of semen, the following issues should be considered.

\section{Resources}

- Physical security of the vessels, specimens and storage room, to reduce risk of loss by theft or fire, or failure of cryopreservation straws, ampoules and vessels, or liquid nitrogen supply.

- Suitability of equipment for proposed use.

- System of containment and removal of nitrogen.

\section{Staff safety and protection}

- Personal protective equipment.

- Alarm systems for detection of low liquid nitrogen and low atmospheric oxygen levels.

\section{Risk of cross-contamination}

To reduce the risk of cross-contamination with infectious agents between samples in storage (e.g. transmission of HIV, or hepatitis B or C via a cryopreservation vessel), consider:

- type of storage container: vials or straws and method of sealing straws (heat or polymer);

- $\quad$ nature of storage: liquid nitrogen or vapour phase;

- protocol and method of storage of high-risk samples (samples known or suspected to contain viruses).

\section{Security of frozen samples}

- $\quad$ Split samples and store at different sites to reduce risk of total loss.

- Double-check identity of samples at each step.

- Use robust labelling and identifying codes.

- Have procedures for regular audit of use of material and samples remaining in storage.

Sources: Tedder, 1995; Mortimer, 2004; Gilling-Smith et al., 2005; Tomlinson, 2005. 
Note 1: Storage in the vapour phase rather than in liquid nitrogen itself may reduce the chances of cross-contamination. However, large temperature gradients can exist in vapour storage vessels, depending on the shape, sample load and type of sample containers. In extreme cases, a temperature of less than $-100{ }^{\circ} \mathrm{C}$ cannot be achieved (Tomlinson, 2005). If vapour phase storage is used, care is needed to ensure that the temperature of the samples does not go above $-130^{\circ} \mathrm{C}$ (the glassy transformation temperature) as this may result in damage to the spermatozoa (see Clarke, 1999).

Note 2: Secure straws made from heat-sealable ionomeric resin are available for storage in liquid nitrogen. These are leak-proof, bacteria- and virus-proof, and mechanically resistant at $-196{ }^{\circ} \mathrm{C}$ (M ortimer, 2004; Gilling-Smith et al., 2005; Tomlinson, 2005).

\subsection{Semen cryopreservation protocols}

Several freezing and sperm bank management protocols are available (Mortimer, 2004; Wolf, 1995). Several cryoprotectants are available commercially. Details of a commonly used cryoprotectant, glycerol-egg-yolk-citrate (GEYC), and machinecontrolled or vapour freezing are given below.

\subsubsection{Standard procedure}

\subsubsection{Preparing the GEYC cryoprotectant}

1. To $65 \mathrm{ml}$ of sterile purified water add $1.5 \mathrm{~g}$ of glucose and $1.3 \mathrm{~g}$ of sodium citrate tribasic dihydrate.

2. Add $15 \mathrm{ml}$ of glycerol and mix thoroughly.

3. Add $1.3 \mathrm{~g}$ of glycine. When dissolved, filter the solution through a $0.45-\mu \mathrm{m}$ pore filter.

4. Add $20 \mathrm{ml}$ of fresh egg yolk (preferably obtained from specific pathogen-free eggs): wash the egg and remove the shell. Pierce the membrane surrounding the yolk and take up into a syringe (approximately $10 \mathrm{ml}$ of yolk will be obtained per egg).

5. Place the whole suspension in a water-bath at $56{ }^{\circ} \mathrm{C}$ for 40 minutes with occasional swirling.

6. Check the pH of the solution. If it is outside the range 6.8-7.2, discard the solution and prepare a new one, in case incorrect ingredients or amounts were added.

7. Bacterial culture for sterility testing can be performed at this stage.

8. Testing for sperm toxicity can be performed at this stage.

9. Dispense the solution in 2- $\mathrm{ml}$ aliquots in a sterile work cabinet and store at $-70{ }^{\circ} \mathrm{C}$. 
10. Use within 3 months.

Cryoprotectants similar to GEYC are commercially available.

\subsubsection{Adding cryoprotectant to semen}

1. Thaw the cryoprotectant, warm to room temperature and mix. Initial warming to $37^{\circ} \mathrm{C}$ may be beneficial.

2. High concentrations of glycerol are detrimental to spermatozoa. It is thus vital to take special care when adding and mixing the cryoprotectant with the semen.

3. Add one volume of GEYC to two volumes of semen, either drop by drop with swirling, or by gentle pipetting up and down, or gradually in five additions with gentle mixing over approximately 10 minutes at room temperature.

4. After the GEYC has been added, incubate the mixture at $30-35{ }^{\circ} \mathrm{C}$ for 5 minutes.

\subsubsection{Filling semen straws}

1. Plastic $0.5-\mathrm{ml}$ straws are popular because of their heat transfer properties and ease of storage. Plastic vials may be used for storing larger volumes.

2. Aspirate the semen-GEYC mixture into $0.5 \mathrm{ml}$ plastic semen straws or place in cryovials. Straws can be filled with a manifold on a vacuum device or an adaptor that fits over the end of the straw.

\subsubsection{Sealing semen straws}

Straws with an upper plug of dry polyvinyl alcohol powder held between two sections of cotton wool automatically seal when the semen makes contact with and polymerizes the powder.

1. Leave a 1-cm air space at the lower end by tapping the straw on the side of the container.

2. Close this end by dipping in sterile polyvinyl alcohol sealing powder and placing the straws in water to a depth of $1 \mathrm{~cm}$.

3. Heat-sealing the straws may be preferable, as the powder seals may be permeable to infectious agents.

4. Alternatively, the samples may be stored in plastic vials or ampoules. They should be filled to not more than $90 \%$ of their capacity.

5. Wipe the outside of the container dry and then sterilize with $70 \%(\mathrm{v} / \mathrm{v})$ alcohol or other mic robial decontaminant.

\subsubsection{Cooling and freezing the semen in programmable freezers}

Programmable freezers are available that control the injection of liquid nitrogen vapour into the freezing chamber. 
1. Place the straws or cryovials in a programmable freezer and follow the manufacturer's instructions to activate the programme.

2. A common programme is to cool the straws at $1.5^{\circ} \mathrm{C}$ per minute from $20^{\circ} \mathrm{C}$ to $-6{ }^{\circ} \mathrm{C}$ and then at $6{ }^{\circ} \mathrm{C}$ per minute to $-100^{\circ} \mathrm{C}$. This takes about 40 minutes. The machine will then hold the chamber at $-100{ }^{\circ} \mathrm{C}$ for 30 minutes to allow for delays before the straws are transferred to liquid nitrogen.

3. Other, more complic ated, procedures may be used, depending on experience in individual laboratories (Pérez-Sánchez et al., 1994).

\subsubsection{Cooling and freezing the semen manually}

Manual methods are less controllable than programmable freezers but can give adequate results. There are many alternatives to this procedure.

1. Place the straws in a refrigerator freezer $\left(-20^{\circ} \mathrm{C}\right)$ for 30 minutes, then on dry ice $\left(-79^{\circ} \mathrm{C}\right)$ for 30 minutes before placing in liquid nitrogen $\left(-196^{\circ} \mathrm{C}\right)$.

2. The straws may be moved from the $-20^{\circ} \mathrm{C}$ freezer into another freezer at $-70{ }^{\circ} \mathrm{C}$, or into a basket or goblet in a mixture of liquid nitrogen vapour and air in the neck of a small liquid nitrogen container at $-80{ }^{\circ} \mathrm{C}$ to $-100{ }^{\circ} \mathrm{C}$ for $10-15$ minutes, before being placed in liquid nitrogen. They can also be placed on a rack $10-20 \mathrm{~cm}$ above liquid nitrogen in a large container, and left for 1 hour to develop a temperature gradient above the liquid nitrogen.

\subsubsection{Storage of frozen semen}

1. Place the frozen straws in plastic storage tubes (mini-goblets) and insert these in larger storage goblets.

2. Place cryovials in clips on metal canes or in storage boxes that fit into the storage tanks, preferably in the vapour phase, because cryovial lids do not provide a complete seal.

3. Store the goblets with the straws or wands in liquid nitrogen vacuum (Dewar) flasks or tanks.

\subsubsection{Transport of frozen semen}

Frozen spermatozoa can be transported in commercially available dry shipper tanks cooled with liquid nitrogen. Depending on the size of the shipper, suitably low temperatures can be maintained for several days to several weeks, as the liquid nitrogen evaporates.

Note: Ensure that local, national and international regulations on shipping liquid nitrogen and human biological samples are complied with.

\subsubsection{Thawing of frozen semen}

1. Before use, remove as many straws as required from the liquid nitrogen or vapour tank and place them on tissue paper or in a rack to allow them to reach 
room temperature (this takes about 6 minutes). Cryovials take longer to thaw (10-20 minutes).

2. Within 10 minutes, cut off the end of the straw with sterile scissors and load the insemination device (for therapeutic use) or expel the contents to determine post-thaw motility (for checking the freezing process).

3. More rapid thawing may be better if the freezing process is rapid (Verheyen et al., 1993).

4. Removing cryoprotectant by sequential dilution in small-volume steps avoids undue osmotic stresses (Gao et al., 1995) and may improve pregnancy results.

6.2.2 Modified freezing protocols for oligozoospermic samples and surgically retrieved spermatozoa

- Semen that contains only a few motile spermatozoa, and sperm suspensions obtained from the genital tract, can be stored for subsequent ICSI.

- If necessary, centrifuge the semen at $1500 \mathrm{~g}$ for 10 minutes to concentrate the spermatozoa into a minimum volume of about $0.4 \mathrm{ml}$. Add GEYC and process as described above.

- Epididymal fluid, testicular extracts or other sperm suspensions processed in the laboratory by swim-up or centrifugation on density gradients (see Sections 5.4 and 5.5) and resuspended in a sperm preparation medium with Hepes buffer and human serum albumin $4 \mathrm{mg} / \mathrm{ml}$ can be cryopreserved with Tyrode's glucose glycerol (TGG) cryoprotectant, or a commercial cryoprotectant containing human albumin.

\subsubsection{Modified cryoprotectant (TGG)}

1. To $40 \mathrm{ml}$ of sterile Tyrode's solution (see Appendix 4, section A4.9) add $5 \mathrm{ml}$ of sterile human albumin stock $(100 \mathrm{mg} / \mathrm{ml}), 0.9 \mathrm{~g}$ of glucose and $5 \mathrm{ml}$ of glycerol. Filter the solution through a $0.45-\mu \mathrm{m}$ pore filter.

2. Store in $2-\mathrm{ml}$ aliquots at $-70^{\circ} \mathrm{C}$.

\subsubsection{Procedure}

1. If the sample volume is greater than $2.0 \mathrm{ml}$, and if few motile spermatozoa are present, centrifuge at $1500 \mathrm{~g}$ for 5 minutes at room temperature.

2. Aspirate the supernatant to leave about $1.0 \mathrm{ml}$ and resuspend the spermatozoa in it. Determine the percentage of motile spermatozoa (PR +NP); if very few motile spermatozoa are present, estimate the number of motile cells under each coverslip.

3. Thaw a 2-ml aliquot of TGG.

4. Add one volume of TGG to one volume of final sperm preparation, gradually, with mixing. 
5. Package in straws or cryovials and freeze as above. If any straws are not full, cap the mini-goblet to prevent the straws from floating when frozen.

\subsubsection{Labelling of straws and records}

A robust coding system for labelling straws or vials is essential. Use the code in all laboratory data sheets and computer databases to maintain the anonymity of donors. Keep the key to the code with the identity of the donor separately and securely. There are many potential coding systems; the important requirement is to have a unique code for each donor or storage client. The following coding system works satisfactorily.

- Each new anonymous donor is allocated a two letter code (AA, AB, AC ... BA ... etc., ending with $Z Z$, after which a new method is needed).

- A three-letter code system is used for patients and known donors: $A A A, A A B$, etc.

- Each specimen from a particular donor is indicated by a number following his personal code. For example, the eighth donation given by donor BT is labelled BT-8.

- The letter code and specimen number should be written on each straw or vial using a black indelible marker. Alternatively, use a printed label designed for use in liquid nitrogen.

- The mini-goblet in which the straws are stored should also contain a marker stick with the code and specimen number.

- Colour-coding of goblets, mini-goblets, straws and sealing powder is also useful for rapid identification.

- As the stored spermatozoa are used the tally of straws or vials is adjusted in the database.

Note: All procedures involving the identity of donor or patient samples, including receipt of samples, preparation and labelling of straws, placement in tanks and thawing of straws for use or discarding, should be double-checked by two people and evidence of this checking witnessed in the laboratory records. Ideally a technician should process only one semen sample at any given time. 
PART III.

Quality assurance 



\section{CHAPTER 7 Quality assurance and quality control}

\subsection{Controlling for quality in the andrology laboratory}

Andrology laboratories need to produce reliable results for appropriate diagnostic and health care decisions. Since semen analysis is highly complex and procedurally difficult to standardize, quality control (QC) is essential to detect and correct systematic errors and high variability of results. The large discrepancies between assessments of sperm concentration and morphology in different laboratories (Neuwinger et al., 1990; Matson, 1995; Cooper et al., 1999, 2002) underline the need for improved QC and standardization.

Whatever its size, each laboratory should implement a quality assurance (QA) programme, based on standardized methods and procedures, to ensure that results are both accurate and precise (De J onge, 2000; Mortimer \& Mortimer, 2005). In some countries, QA programmes are required by law, in others, by accreditation bodies or health insurance systems. In certain settings, the available resources may not permit full implementation of the procedures described here. Nevertheless, the fundamental parameters of sperm concentration, morphology and motility should always be monitored by internal quality control and, where possible, by external quality control.

There are several books describing quality control (e.g. Wheeler $\&$ Chambers, 1992; Wheeler, 1993) and some specializing in laboratory quality control that provide a more in-depth description of the QC process (e.g. Cembrowski \& Carey, 1989; Carey \& Lloyd, 1995; Westgard, 2002). QC activities performed within one laboratory are referred to as internal quality control (IQC) (see Section 7.6). External quality control (EQC) is the evaluation of results from several laboratories for the same samples (see Section 7.11).

\subsection{The nature of errors in semen analysis}

The management of $\mathrm{QC}$ procedures requires an understanding of the source and magnitude of measurement errors. Any measurement has a degree of error, the magnitude of which is described by a confidence interval with an upper and a lower limit. A precise measurement is one in which the limits lie close together; a result is accurate when it is close to the true value. There are two classes of error: random and systematic. Random errors, which affect precision, arise from chance differences in readings or sampling, and can be assessed from repeated measurements by the same observer and equipment. Systematic errors (sometimes referred to as bias) are more insidious, since they arise from factors that alter the result in one direction only, and thus cannot be detected from repeated measurements.

Even when the sample is well mixed, the random distribution of spermatozoa in semen, or in fixative or medium, accounts for much of the lack of precision of the results of semen analysis. The assessment of sperm concentration, motility, vitality and morphology involves counting a limited number of spermatozoa, which are presumed to be representative of the whole sample. The sampling variation created by selecting either a fixed volume (for estimating concentration) or a fixed number of spermatozoa (for classifying motility, morphology or vitality) is a random 
error commonly referred to as the statistical or sampling error. Some terminology in this area is given in Box 7.1. Further errors may be introduced when the sample is mixed or aliquots are removed; these can be minimized by improving technique (see Section 7.13).

The aim of quality control in routine semen analysis is to monitor the extent of both random and systematic errors and reduce it where possible. All these errors need to be minimized for the results to be believable and of use to clinicians and researchers.

\subsection{Minimizing statistical sampling error}

While sampling error can be reduced by assessing greater numbers of spermatozoa (see Table 2.2 and Boxes 2.5 and 2.7), a balance must be struck between the gain in statistical precision, the actual time required to gain it, and the possible loss of accuracy in the technician's work due to fatigue. Using 95\% confidence intervals for assessing the acceptability of replicates means that, for about $5 \%$ of samples, differences greater than 1.96 ×standard error will occur as a result of chance variation alone, and the measurement will have been repeated unnecessarily. This additional work may be acceptable; alternatively, wider limits ( $2.6 \times$ or $3 \times$ standard error) could be chosen to reduce the frequency of this event (to approximately $1 \%$ and $0.2 \%$, respectively).

\section{Box 7.1 Terminology in quality assurance and quality control}

accuracy

assigned value

bias

binomial distribution

Bland-Altman plot

common cause variation

95\% confidence interval

consensus value conventional true value control chart

control limits

drift
Closeness of the agreement of a test result with the true value. Estimate of true value, often derived from the mean of results from a number of laboratories (target value, consensus value, conventional true value).

The deviation of a test result from the assigned value. Reproducible inaccuracies that are consistently in the same direction (systematic error).

A theoretical distribution used to model events falling into two categories, e.g. motile/immotile, viable/non-viable.

A plot of the difference between a series of paired observations against their mean value.

A source of natural variation that affects all individual values of the process being studied.

An interval calculated from observed data that includes the true value in $95 \%$ of replicates (mean $\pm 1.96 \times \mathrm{SE}$ or $\mathrm{N} \pm 1.96 \times \sqrt{ } \mathrm{N}$ for counts).

see assigned value.

see assigned value.

A time-sequence chart showing a series of individual measurements, together with a central line and control limits.

The maximum allowable variation of a process due to common causes alone. Variation beyond a control limit is evidence that special causes may be affecting the process.

Successive small changes in values leading to a change in accuracy with time. 


external quality control
good laboratory practice (GLP
in control
internal quality control
ISO
manufactured QC samples
out of control
PDCA
Poisson distribution
precision

precision error random error $S$ chart sampling error

Shewhart cycle
special cause variation

standard operating procedures statistical sampling error systematic error target value variation $X_{\text {bar }}$ chart Youden plot
Quality tests performed by an external body that makes comparisons between different laboratories for several procedures. Useful for detecting systematic variation and assessing accuracy.

A set of principles that provides a framework within which laboratory studies are planned, performed, monitored, recorded, reported and archived.

A process is in control when all values are within expected control limits.

Quality tests measuring the variability in a procedure that exists within a laboratory. Such tests evaluate the precision of day-to-day operations. Useful for detecting random variation (assessing precision).

International Organization for Standardization. A body that sets international standards, including for laboratory quality.

Commercially available samples, manufactured and analysed (assayed) according to manufacturing guidelines.

A process is out of control when a measured value exceeds expected control limits, or is within control limits but shows a significant trend in values. A process that is out of control must be evaluated. Plan, do, check, act (Shewhart cycle).

A theoretical distribution used to model counts.

Closeness of agreement between replicate measurements. Commonly expressed as imprecision (drift; within-, between-, inter-/run, batch, assay, or laboratory variation). Measurements of precision are not affected by bias (see also sampling error).

see sampling error.

see sampling error.

A control chart of standard deviations of measured values against time. It is used to monitor process uniformity and measurement precision.

The error involved in counting a limited number of spermatozoa; it is inversely proportional to the square root of the number counted. The sampling error $(\% \mathrm{SE})$ is the standard error of a count $(\sqrt{ } \mathrm{N})$ expressed as a percentage of the count $(100 \times(\sqrt{ } \mathrm{N} / \mathrm{N}))$. (random error, precision error, statistical sampling error).

see PDCA.

A source of variation that is large, intermittent or unpredictable, affecting only some of the individual values of the process being studied (random variation).

Set of instructions for how processes and methods should be carried out.

see sampling error.

see bias.

see assigned value.

The difference between individual results of a process. The cause of variation (error) can be common or special.

A control chart showing means of measured values against time. It is used to monitor process variability and detect changes from the target values (assessing accuracy).

A graph of values from one sample plotted against another. 


\subsection{The quality assurance programme}

The best way to achieve acceptable results is to develop and implement a continuous QA programme. A Q A programme monitors and evaluates, on a regular basis, the quality and appropriateness of the data and services that the laboratory provides. Management, administration, statistical analysis, and preventive and corrective action form the core of the QA plan. Continuous monitoring not only detects and corrects problems, but also helps prevent them.

The QA programme should be described in a quality manual (QM) containing standard operating procedures (SOPs) and a detailed set of instructions for the different processes and methods used in the laboratory. Linked to these instructions are a number of forms and documents, such as referral notes, laboratory worksheet report forms, and information leaflets to clients and referring clinicians. The QM describes the organizational structure of the laboratory, listing the required skills (training) needed in different positions (job descriptions), as well as schedules for meetings between testing personnel and supervisors, and plans for continuous education, development and training of staff.

\subsection{Laboratory procedures manual}

The written SOPs should be strictly followed by all laboratory technicians. They are also useful for training and are an important reference for non-routine procedures and for troubleshooting processes that are not producing acceptable results.

These protocols include referral notes, patient information procedures, schedules of patient appointments, performance of assays, reporting of analytical results, training of new laboratory staff members, testing and monitoring of equipment, use of control charts and procedures to follow when values on these charts indicate a problem (out-of-control assays). SOPs should cover procedures for checking that all equipment is in proper operating condition, including routine checking of operation, a schedule and log of calibration, and documentation on the maintenance of scientific equipment, such as microscopes, centrifuges, pipettes, balances, freezers, refrigerators and emergency equipment (e.g. eye washes and showers). The basic method is to keep a log book for each piece of equipment, in which all adjustments and calibrations are recorded. These records are useful if a laboratory procedure starts producing out-of-control results.

\subsection{Internal quality control}

Internal quality control (IQC) monitors precision and indicates, through results outside the control limits, when the assay may be faulty. The QC procedure used depends on the assessment to be controlled, since different assessments are sensitive to different types of errors. Assessments that involve dilution, pipetting and reuse of chambers require regular testing, whereas an assessment of a fixed slide or videotape may be tested less often, as there are fewer steps where errors can occur. 
A practical way to implement IQC is to include IQC materials in the laboratory's regular workload and to monitor the results for these materials using quality control charts. In this way, IQC becomes part of the laboratory routine and is conducted according to local or regional standards. It is important that QC samples are analysed as part of routine laboratory work and not treated in a special way, which could provide a more precise and accurate result than that for routine samples. The types of IQC material used to monitor within- and between-technician variation can be purchased or made in the laboratory; there are advantages and disadvantages of each approach.

\subsubsection{Purchased QC samples}

Commercially available IQC samples are provided with a mean and known extent of variation established for that product. The advantage of these is that both accuracy and precision can be evaluated. The variation in semen analysis results in the laboratory can be compared with the variation associated with samples from the approved source. With such samples, the laboratory should establish its own control chart for assessing precision and should use the manufacturer's recommended range for evaluating accuracy (Westgard, 2002). The disadvantages of purchased IQC samples are their cost and the fact that they are not universally available. A note should be made of how the target values given by the manufacturer were obtained (multiple assessments, computer-aided sperm analysis, consensus values, trimmed means, etc.).

\subsubsection{Laboratory-made QC samples}

The advantages of laboratory-produced IQC samples are the reduced costs and the fact that the samples can be generated specifically for the laboratory's particular needs. Many samples, covering a broad range of results, can be prepared and stored for long periods. Their disadvantage is that the target values are unknown. It is recommended, and sometimes required, that there be control samples for evaluating an average range of values (e.g. sperm concentration $50 \times 10^{6}$ per ml) as well as a critical range of values (e.g. sperm concentrations $<15 \times 10^{6}$ per $\mathrm{ml}$ ).

\subsubsection{Stored samples (purchased or laboratory-made)}

Stored semen samples can be used in IQC programmes for assessing sperm concentration, sperm motility, sperm morphology and sperm vitality. These have the advantage that the target value is known (for purchased samples), or provided (by EQC programmes) or estimated from multiple assessments (for laboratoryproduced material), so that systematic errors can be detected from repeated measurements.

\subsubsection{Sperm concentration}

Semen samples of varying sperm concentrations can be diluted and stored. Several specimens may be pooled to achieve a certain concentration or a larger volume of diluted suspension, but sperm agglutination may occur.

See Appendix 7, section A7.6, for instructions on preparing and storing non-agglutinated sperm suspensions for IQC of measurement of sperm concentration. 


\subsubsection{Sperm morphology and vitality}

For morphology, slides of air-dried, fixed semen smears (see Section 2.13.2.1) or fixed and stained semen smears (see Section 2.14), and for vitality, eosin-nigrosin smears (see Section 2.6.1) can be used. Smears should be chosen from the laboratory's routine samples, with identifying codes masked. Samples should be prepared from semen of good, medium and poor quality. The slides can be reused; once they begin to deteriorate, new ones should be prepared. It is best to use a range of slides to eliminate the possibility of technicians becoming familiar with certain slides, which may result in biased analyses.

See Appendix 7, section A7.7, on how to prepare slides for QC of morphology assessment. If slides are prepared and stored properly, they remain stable for many months or even years. Different slide sets can be alternated or overlapped with each other during transition from one QC set to another.

\subsubsection{Sperm motility}

Video-recorded specimens on tape, CD or DVD, from the clinic, from EQC distributions, or specifically made, can be used for QC. Video-recordings should be of a magnification similar to that observed in the microscope when actual specimens are analysed. The use of a television camera and screen for all daily routine assessments, at the same magnification and contrast as the video-recordings, increases the validity of video-recordings for $\mathrm{QC}$.

See Appendix 7, section A7.5, on how to make video recordings for QC of motility measurements.

\subsubsection{Fresh QC samples (laboratory-made)}

A simple method of IQC is for one or more technicians to make replicate measurements on separate aliquots of a semen sample. The replicate assessments should be performed in the same way as routine semen analyses. This form of QC can be applied to assessments of sperm concentration, sperm motility, sperm morphology and sperm vitality. The subjective nature of assessments of agglutination and aggregation, and the variability of the mixed antiglobulin reaction test (Bohring \& Krause, 1999), together with the need for live gametes and positive controls, make QC for these assays difficult.

The IQC of measurement of sperm motility in fresh samples presents special problems, since motility may decline over time, and thus needs to be assessed first-and at about the same time-by all the technicians. Slide and coverslip preparations for motility are stable for only a few minutes, so fixed-depth chambers, which are stable for 30 minutes, can also be used. Use of a bridge microscope, or a microscope with a video camera linked to a screen, allows several technicians to assess the same field from the same preparation at the same time. An acetate grid can be placed over the monitor to mimic the ocular grid used during live motility analysis (see Appendix 7, section A7.5). 
Laboratories using CASA systems should follow the manufacturers' procedures for conducting quality control. This often involves replaying stored images of moving spermatozoa that are marked as swimming at certain velocities.

\subsection{Statistical procedures for analysing and reporting within- and among-technician systematic errors}

The creation and interpretation of control charts are an integral part of quality assurance in the laboratory. Which QC systems are used depends on the nature of the problem and of the material available.

7.7.1 The $X_{\text {bar }}$ chart

The $X_{b a r}$ chart is designed primarily to detect results that are very different from the target value, or an overall increase in variation. Systematic errors can be detected by sequential measurement of the same samples. Repeated measurements are made on a sample and the mean values plotted against time. Stored samples need to be used as the procedure depends on knowing the true or target value, which may be provided by the manufacturer (purchased samples), or an EQC programme, or estimated (from multiple assessments of the material).

Comment: The $\mathrm{X}_{\mathrm{bar}}$ chart is less sensitive than the $\mathrm{S}$ chart in detecting whether technicians are producing highly variable results (see Section 7.7.2). To check variability, the range of values for each $\mathrm{QC}$ sample can be monitored on an $\mathrm{S}$ chart in a similar way to the $X_{\text {bar }}$ chart, with warning and action limits set accordingly.

7.7.1.1 Calc ulating the control limits of the $X_{\text {bar }}$ chart

A series of QC samples from the same IQC preparation is measured sequentially. After the first 10 samples have been analysed, the control limits are calculated for each technician. These depict the range for repeated measurements on a sample, for a specific procedure performed by the same analysts. The estimates of the mean and the standard deviation are recomputed after every 10 samples and the control limits updated using the new values for $X_{b a r}$ and $S_{b a r}$, provided there have been no problems with $\mathrm{QC}$. Before the $\mathrm{QC}$ samples run out, a new pool should be prepared and the first 10 samples of the new batch analysed together with the remaining samples of the old batch to establish the new control limits. The factors used to compute the control limits are given in Table 7.1 and worked examples are shown in Boxes 7.2 and 7.3. 
Table 7.1 Factors for determining control limits for $\mathrm{X}_{\mathrm{bar}}$ charts and $\mathrm{S}$ charts based on the average standard deviation $\left(\mathrm{S}_{\text {bar }}\right)$

\begin{tabular}{|c|c|c|c|c|c|c|c|}
\cline { 3 - 8 } \multicolumn{2}{c|}{} & \multicolumn{2}{c|}{$\mathrm{X}_{\text {bar }}$ control limits } & \multicolumn{4}{c|}{$\mathrm{S}_{\text {bar }}$ control limits } \\
\hline $\begin{array}{c}\text { No. of } \\
\text { technicians } \\
(\mathrm{n})\end{array}$ & $\begin{array}{c}\mathrm{SD} \\
\text { estimate } \\
\left(\mathrm{C}_{\mathrm{n}}\right)\end{array}$ & $\begin{array}{c}\text { Warning } \\
\text { limit } \\
\left(\mathrm{A}_{2}\right)\end{array}$ & $\begin{array}{c}\text { Action } \\
\text { limit } \\
\left(\mathrm{A}_{3}\right)\end{array}$ & $\begin{array}{c}\text { Lower } \\
\text { action } \\
\text { limit }\left(\mathrm{S}_{0.999}\right)\end{array}$ & $\begin{array}{c}\text { Lower } \\
\text { warning } \\
\text { limit }\left(\mathrm{S}_{0.975}\right)\end{array}$ & $\begin{array}{c}\text { Upper } \\
\text { warning } \\
\text { limit }\left(\mathrm{S}_{0.025}\right)\end{array}$ & $\begin{array}{c}\text { Upper } \\
\text { action limit } \\
\left(\mathrm{S}_{0.01}\right)\end{array}$ \\
\hline 2 & 1.253 & 1.772 & 2.659 & 0.002 & 0.039 & 2.809 & 4.124 \\
\hline 3 & 1.128 & 1.303 & 1.954 & 0.036 & 0.180 & 2.167 & 2.966 \\
\hline 4 & 1.085 & 1.085 & 1.628 & 0.098 & 0.291 & 1.916 & 2.527 \\
\hline 5 & 1.064 & 0.952 & 1.427 & 0.160 & 0.370 & 1.776 & 2.286 \\
\hline 6 & 1.051 & 0.858 & 1.287 & 0.215 & 0.428 & 1.684 & 2.129 \\
\hline 7 & 1.042 & 0.788 & 1.182 & 0.263 & 0.473 & 1.618 & 2.017 \\
\hline 8 & 1.036 & 0.733 & 1.099 & 0.303 & 0.509 & 1.567 & 1.932 \\
\hline 9 & 1.032 & 0.688 & 1.032 & 0.338 & 0.539 & 1.527 & 1.864 \\
\hline 10 & 1.028 & 0.650 & 0.975 & 0.368 & 0.563 & 1.495 & 1.809 \\
\hline
\end{tabular}

Box 7.2 Determining the values for the warning and action control limits of an $X_{b a r}$ chart

The table below shows the sperm concentrations measured by four technicians on 10 QC samples from the same IQC preparation, together with the calculated mean and standard deviation for each sample.

$\begin{array}{lllllllllll}\text { Sample: } & 1 & 2 & 3 & 4 & 5 & 6 & 7 & 8 & 9 & 10\end{array}$ Sperm concentration $\left(10^{6}\right.$ per $\left.\mathrm{ml}\right)$

$\begin{array}{lllllllllll}\text { Technician A: } & 38 & 35 & 40 & 34 & 38 & 36 & 44 & 43 & 39 & 43\end{array}$

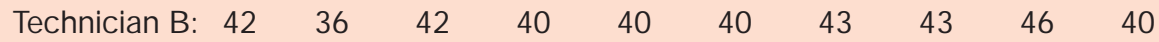

Technician C: $\begin{array}{llllllllll}38 & 43 & 40 & 51 & 38 & 33 & 39 & 45 & 35 & 39\end{array}$

Technician D: $\begin{array}{llllllllll}34 & 36 & 36 & 37 & 36 & 39 & 42 & 43 & 46 & 34\end{array}$

$\begin{array}{lllllllllll}\text { Mean } & 38.0 & 37.5 & 39.5 & 40.5 & 38.0 & 37.0 & 42.0 & 43.5 & 41.5 & 39.0\end{array}$

$\begin{array}{lllllllllll}\text { SD } & 3.27 & 3.70 & 2.52 & 7.42 & 1.63 & 3.16 & 2.16 & 1.00 & 5.45 & 3.74\end{array}$

For the $10 \mathrm{QC}$ samples, the average of the means $\left(\mathrm{X}_{\mathrm{bar}}\right)$ is:

$(38.0+37.5+\ldots+39.0) / 10=39.7$, and the average of the SDs $\left(S_{\text {bar }}\right)$ is:

$(3.27+3.70+\ldots+3.74) / 10=3.40$.

The values of the coefficients $A_{2, n}$ and $A_{3, n}$ (see Table 7.1) for $n=4$ are 1.085 and 1.628 , respectively. Thus the warning control limits (two standard errors from the mean) are given by:

$X_{\text {bar }} \pm A_{2, n} \times S_{\text {bar }}=39.7 \pm(1.085 \times 3.40)=39.7 \pm 3.7$, or 36.0 and $43.3 \times 10^{6}$ per $\mathrm{ml}$.

Similarly, the action control limits (three standard errors from the mean) are given by: $X_{\text {bar }} \pm A_{3, n} \times S_{\text {bar }}=39.7 \pm(1.628 \times 3.40)=39.7 \pm 5.5$, or 34.2 and $45.2 \times 10^{6}$ per ml. 
B ox 7.3 Alternative method for calculating the $X_{b a r}$ control limits from the pooled standard deviation

The estimate of between-technician standard deviation can also be obtained by multiplying $S_{b a r}$ by $C_{n}(=1.085$ for sample size 4 (Table 7.1)) to give 3.69. This is close to the directly computed value of 3.84 of the pooled standard deviation, $\mathrm{s}=\sqrt{ }\left(\left(\mathrm{s}_{1}{ }^{2}+\mathrm{s}_{2}{ }^{2}+\ldots+\mathrm{s}_{10}{ }^{2}\right) / 10\right)$, where $\mathrm{s}_{\mathrm{i}}$ is the standard deviation of the ith QC sample. This result can be used to compute the warning and action control limits directly, at 2 and 3 standard errors $\left(s / V_{n}\right)$ either side of the mean. In this example, these warning limits are 35.8 and $43.5 \times 10^{6}$ per $\mathrm{ml}$, and the action limits are 33.9 and $45.5 \times 10^{6}$ per $\mathrm{ml}$, respectively - very close to those obtained using $S_{\text {bar }} A_{2, n}$ and $A_{3, n}$.

\subsubsection{Plotting the $X_{b a r}$ chart}

Each technician should analyse the IQC samples and contribute to the IQ C control chart. Once an assay procedure is in place with acceptable variation, IQC samples should be analysed routinely and results compared with the established values. This is done by plotting the mean values measured for the IQC samples in each assay on the control chart and observing if they lie outside the variability (error) determined for the method in the laboratory. See Fig. 7.1 for an example.

$X_{b a r}$ charts can be constructed, and warning and action limits set, for the assessment of sperm motility, morphology and vitality, following the steps outlined for sperm concentration, with the difference that percentages are assessed (see Section 7.8).

Fig. 7.1 An $\mathrm{X}_{\text {bar }}$ chart for sperm concentration

The mean values for sequential measurements $(\diamond)$ are plotted on a graph showing the previously measured target value $\left(\mathrm{X}_{\mathrm{bar}}\right)$ and the warning and action limits.

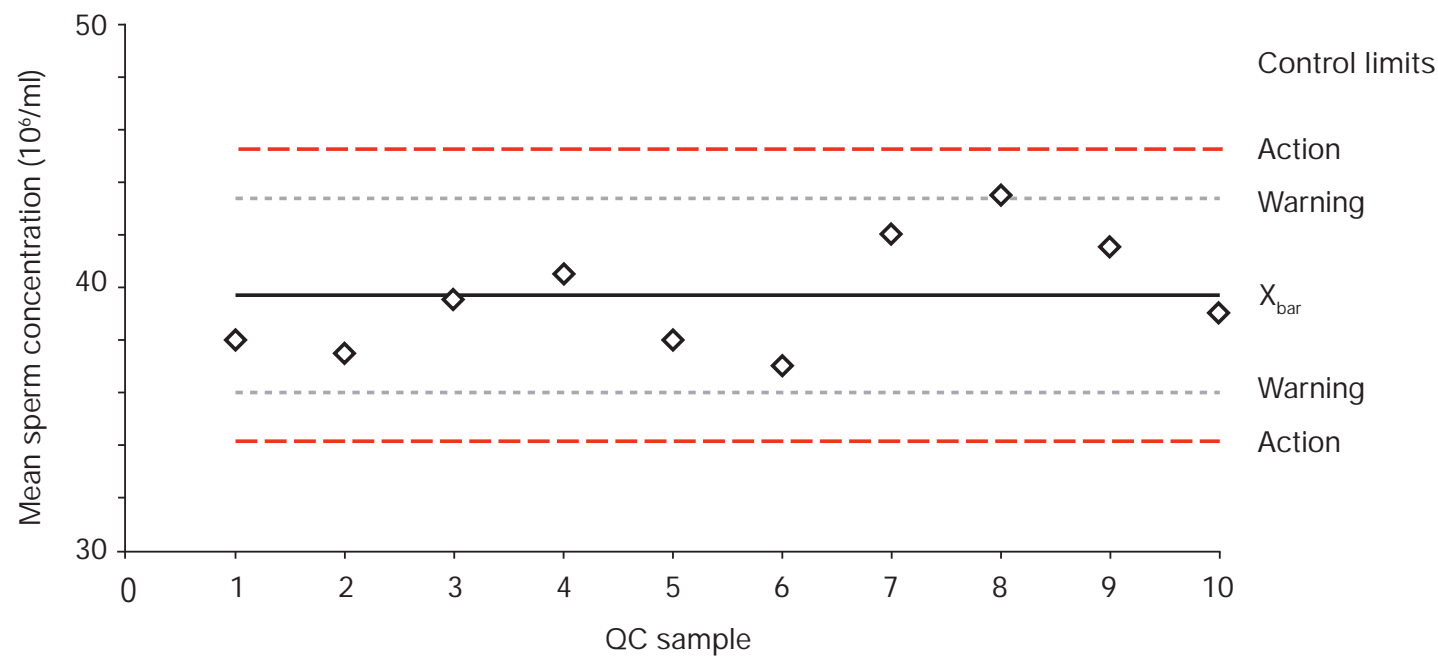




\subsubsection{The S chart}

This chart detects whether technicians are producing highly variable results. Repeated measurements are done and the standard deviations plotted against time. Since the QC samples are all from the same stored pool, no differences between samples are expected, so any significant differences between technicians would suggest systematic bias in the assessment by one or more technicians.

\subsubsection{Calculating the $\mathrm{S}$ chart control limits}

Control limits are added to the $S$ chart in the same way as for $X_{b a r}$ charts. However, since the distribution of the standard deviation is not symmetrical, the warning and action limits are chosen in such a way that the probability that a new observation falls outside the control limits is the same as for the $X_{b a r}$ chart if there are no changes in accuracy or precision. Thus, the warning and action limits will be crossed in $5 \%$ and $0.2 \%$, respectively, of future samples as a result of random variation alone. These limits are determined from the $\chi^{2}$ distribution, and the factors $S_{\alpha, n}$ used to multiply the average standard deviation $S_{\text {bar }}$ are given in Table 7.1. A worked example is shown in Box 7.4. Results that fall below the lower limits on the $S$ chart suggest unexpectedly small variation, which may indicate a genuine improvement in the level of agreement between technicians, or possible collusion.

Box 7.4 Determining the vaues for the warning and action control limits of an S chart

Using the results from Box 7.2, the average sample standard deviation $\mathrm{S}_{\mathrm{bar}}$ is $3.40 \times 10^{6}$ per $\mathrm{ml}$.

The values for $s_{\alpha, n}$ for $n=4$ are read from Table 7.1 to give:

$$
\begin{array}{ll}
\text { the lower action limit } & \mathrm{S}_{\text {bar }} \times \mathrm{S}_{0.999,4}=3.40 \times 0.098=0.33 \times 10^{6} \text { per ml, } \\
\text { the lower warning limit } & \mathrm{S}_{\text {bar }} \times \mathrm{S}_{0.975,4}=3.40 \times 0.291=0.99 \times 10^{6} \text { per ml, } \\
\text { the upper warning limit } & \mathrm{S}_{\text {bar }} \times \mathrm{S}_{0.025,4}=3.40 \times 1.916=6.51 \times 10^{6} \text { per ml, and } \\
\text { the upper action limit } & \mathrm{S}_{\text {bar }} \times \mathrm{S}_{0.001,4}=3.40 \times 2.527=8.59 \times 10^{6} \text { per ml. }
\end{array}
$$

\subsubsection{Plotting the S chart}

Subsequent values for standard deviation are plotted on the control chart to determine whether they lie outside the variability (error) determined for the method in the laboratory. See Fig. 7.2 for an example.

$S$ charts can be constructed, and warning and action limits set, for the assessment of sperm motility, morphology and vitality, following the steps outlined for sperm concentration, with the difference that percentages are assessed (see Section 7.8). 
Fig. 7.2 An S chart for sperm concentration

The standard deviations for sequential measurements $(\diamond)$ are plotted on a graph showing the previously measured mean value $\left(\mathrm{S}_{\mathrm{bar}}\right)$ and the warning and action limits.

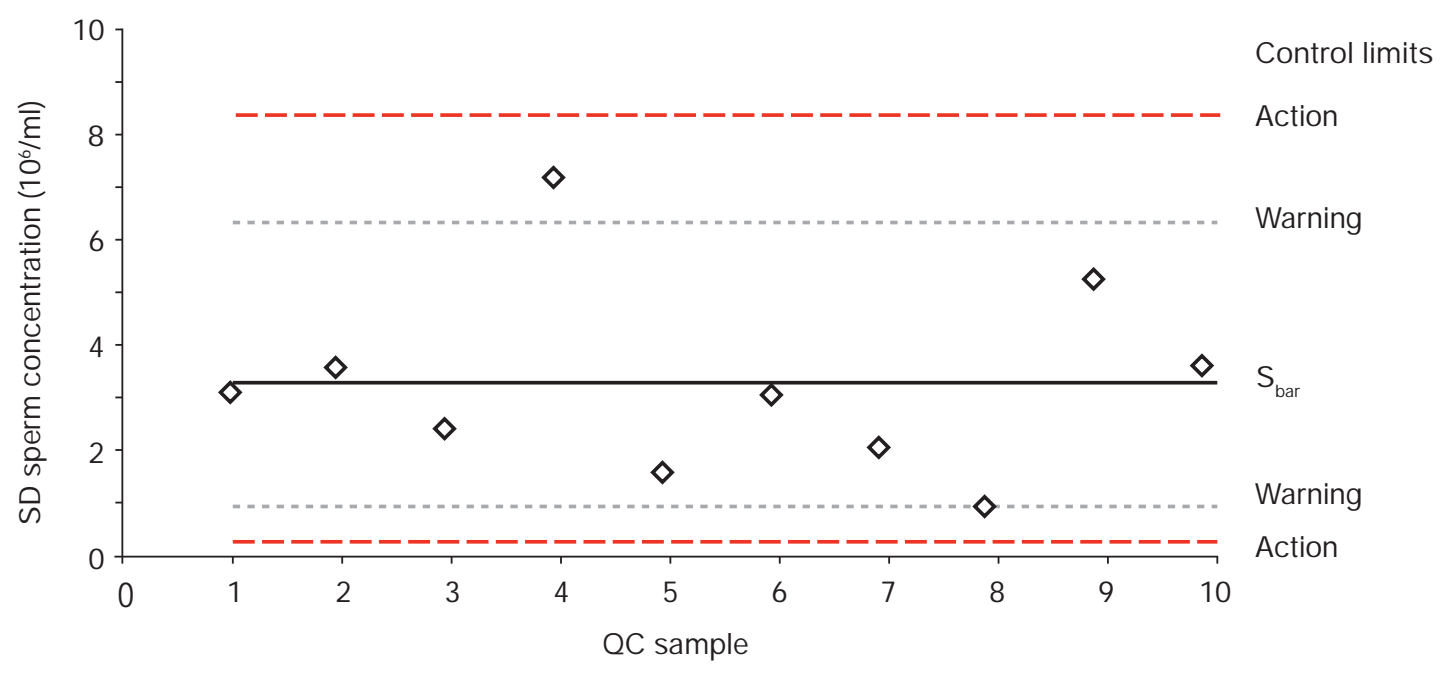

\subsection{QC for percentages}

When spermatozoa are classified into two or more classes (such as normal or abnormal morphology, progressive or non-progressive motility, alive or dead), the standard error of the estimated percentage within a class depends on the true, but unknown, percentage as well as the number of spermatozoa counted $(\mathrm{N})$. The common approximate estimation of standard error of a proportion, $\mathrm{p}$, is $\sqrt{ }(p(100-p) / N)$ for values in the range $20 \%$ to $80 \%$. Outside this range, a more appropriate method to use is the angular transformation (arc sin square root), $z=\sin ^{-1} \sqrt{ }(p / 100)$, for which the standard deviation is $1 /(2 \sqrt{N})$ radians, i.e. dependent only on the number of spermatozoa counted and not the true percentage (see Kuster et al., 2004).

While the standard deviation of individual readings should be close to these values, the average standard deviation $\left(\mathrm{S}_{\text {bar }}\right)$ will exceed $2.5 \%$, because of the additional variation between technicians. In this case the goal will be to reduce $S_{\text {bar. }}$.

\subsection{Assessing $X_{b a r}$ and S charts}

The technicians and laboratory supervisor should review the control charts together. If the control values are not acceptable, a systematic evaluation of the entire procedure should be conducted to determine the possible sources of variation.

7.9.1 How to recognize out-of-control values

There are basic guidelines for monitoring quality control of procedures. The QC charts should be examined in the light of these guidelines, and action taken when indicated. There are various rules for declaring a method to be out of control, including the following: 
- A single point lies outside the 3 SD control limits. This is the simplest rule, and appears to be universally adopted. It may indicate a sudden large shift in the process.

- Two out of three consecutive points lie outside the action control limits.

- Four out of five consecutive points lie outside the warning control limits.

- Two consecutive results lie above the upper, or below the lower, warning control limits.

- Two consecutive results lie one above the upper, and one below the lower, warning control limit.

- Eight consecutive points are on the same side of the centre line. This rule is attractive because it is simple to apply and is sensitive to gradual shifts or trends that the first rule might miss.

In practice, use of the first and last of these rules is generally accepted. If the QC sample is "rejected", the sensitivity of the alarm to the different types of error (random or systematic) should direct the investigation into possible causes (see Box 7.5). The laboratory supervisor should review the $Q C$ results regularly.

\section{Box 7.5 Basic control rules for QC charts}

\section{Control rule}

One result outside action limits

Two out of three points outside the action control limits Four out of five points outside the warning control limits Two consecutive results, both above or both below the upper/lower warning limits

Two consecutive results, one above and one below the upper/lower warning limit

Eight consecutive results, all above or all below the mean
Error indicated

Random

Systematic

Systematic

Systematic

Random

Systematic

\subsubsection{Causes of out-of-control values}

Signals from the QC procedure must be carefully assessed and any procedural errors identified. Possible errors include:

- inadequate mixing of sample (common with viscous and agglutinated samples);

- technician stress (e.g. erratic sampling or recording error);

- poor technique (e.g. careless pipetting or handling during slide or chamber preparation) (see Section 7.13);

- inadequate training (e.g. systematic differences in the identification of spermatozoa for counting, the classification of normal morphology, the assessment of pink and white sperm heads or coiled sperm tails for sperm vitality, and the detection of motile spermatozoa; biases from consistent calculation errors) (see Section 7.13); 
- instrument variation (e.g. worn or uncalibrated automatic pipettes, which may reduce reproducibility during sampling and dilution; misaligned microscopes, which may reduce optical clarity and prevent proper scoring of vitality or morphology; inaccurate balances or measuring cylinders) (see Appendix 7, section $\mathrm{A} 7.8$ );

- deterioration of the QC samples;

- change in equipment, particularly pipettes and counting chambers;

- change in procedures or laboratory environment.

\subsubsection{Responses to out-of-control values}

When results are outside control limits, the probable cause and the corrective action taken should be recorded. If the problem is not obvious, reanalyse the QC samples to check if the first result was unusual. If the $\mathrm{QC}$ result remains outside control limits, the cause must be found and corrected before further assays are performed.

To do this:

- Create a flowchart of the entire process, step by step. The SOP and Tables 7.2-7.5 can aid this process.

- From the flow chart, identify areas of potential variation, deduce possible causes and develop a plan to reduce the variation.

- Collect more data, make new control charts and review them to determine if the variability is acceptable for the procedure. This sequence of identifying a problem, developing and testing a hypothesis, and re-evaluating the process is known as the Shewhart or PDCA (plan, do, check, act) cycle.

\subsection{Statistical procedures for analysing and reporting among- tec hnician variability}

QC procedures based on the assessment of fresh semen samples are similar to those for stored samples and allow the variability within and among technicians to be assessed. However, as the true value is not known, the $X_{b a r}$ chart cannot be used, and systematic error (technician bias) cannot be estimated. Here, the primary QC procedures are the $\mathrm{S}$ chart for assessing variability among technicians, and two-way analysis of variance (ANOVA) for assessing systematic differences among technicians after every five or 10 QC samples.

7.10.1 Comparing results from two or more technicians

Results from two or more technicians can be compared in several ways.

- Plotting the difference between two estimates against their mean (Bland \& Altman, 1986). A comparison of estimates by two technicians of sperm concentration from the same sample should produce a pattern similar to that in Fig. 7.3, where estimates of sperm motility by a technician and a computer are compared. 
Fig. 7.3 A Bland-Altman plot of manual and CASA estimates of percentage progressive sperm motility

The graph plots the difference between results with the two methods (manual - CASA) against the mean ((manual+CASA)/2).

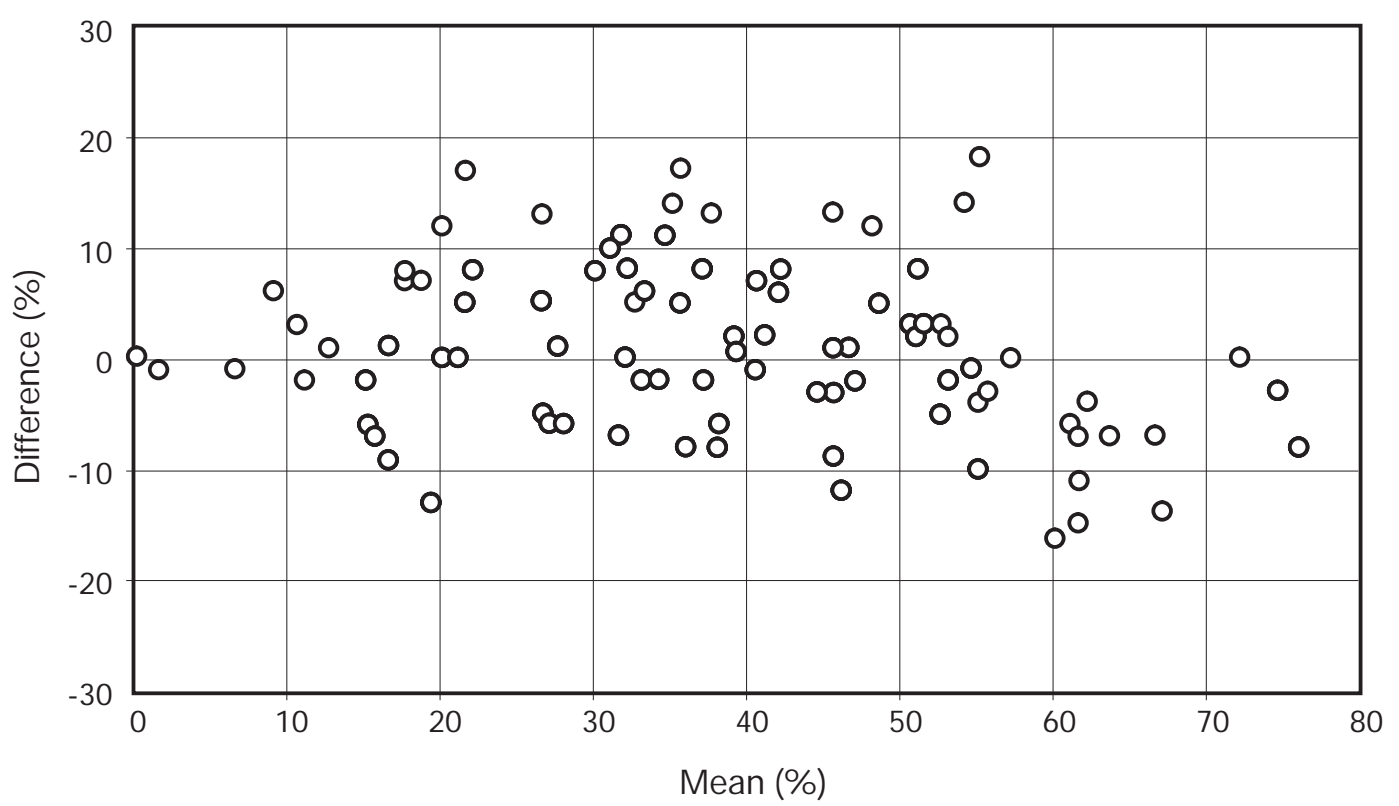

Data courtesy of HWG Baker.

- Calculating the mean and SD of the differences (paired comparisons). As the same sample is analysed by both technicians, the difference between means should be zero. Any significant difference from zero, as assessed by a paired t-test, reveals bias (systematic difference) between the two technicians.

- Plotting results from two samples against each other (Youden plots). A comparison of estimates of concentration by several technicians, each examining two separate specimens, should produce a pattern similar to that in Fig. 7.4. For each technician (for IQC) or each centre (for EQC), the values for the two specimens are plotted against each other. The dotted horizontal and vertical lines indicate the $95 \%$ confidence limits of results from experienced technicians (IQC) or reference laboratories (EQC). The area defined by the intersection of these lines is the target window into which the values should fall. This plot reveals random errors when the value for one sample is in the correct range but the value for the other sample is not (marked 1 ) and systematic errors when both sample estimates are too high (top right panel, marked 2) or too low (lower left panel, marked 2). Random errors most likely contribute to one sample being too low and the other too high (marked 3). 
Fig. 7.4 A Youden plot of estimates of the concentration of spermatozoa

Results from analyses of two samples (A, B ) by several technicians, plotted against each other. The results for each technician (or laboratory in EQC) can be shown by different symbols and colours. Results in panels marked 2 are likely to be due to systematic errors, while those in panels marked 1 and 3 are likely to be due to random errors.

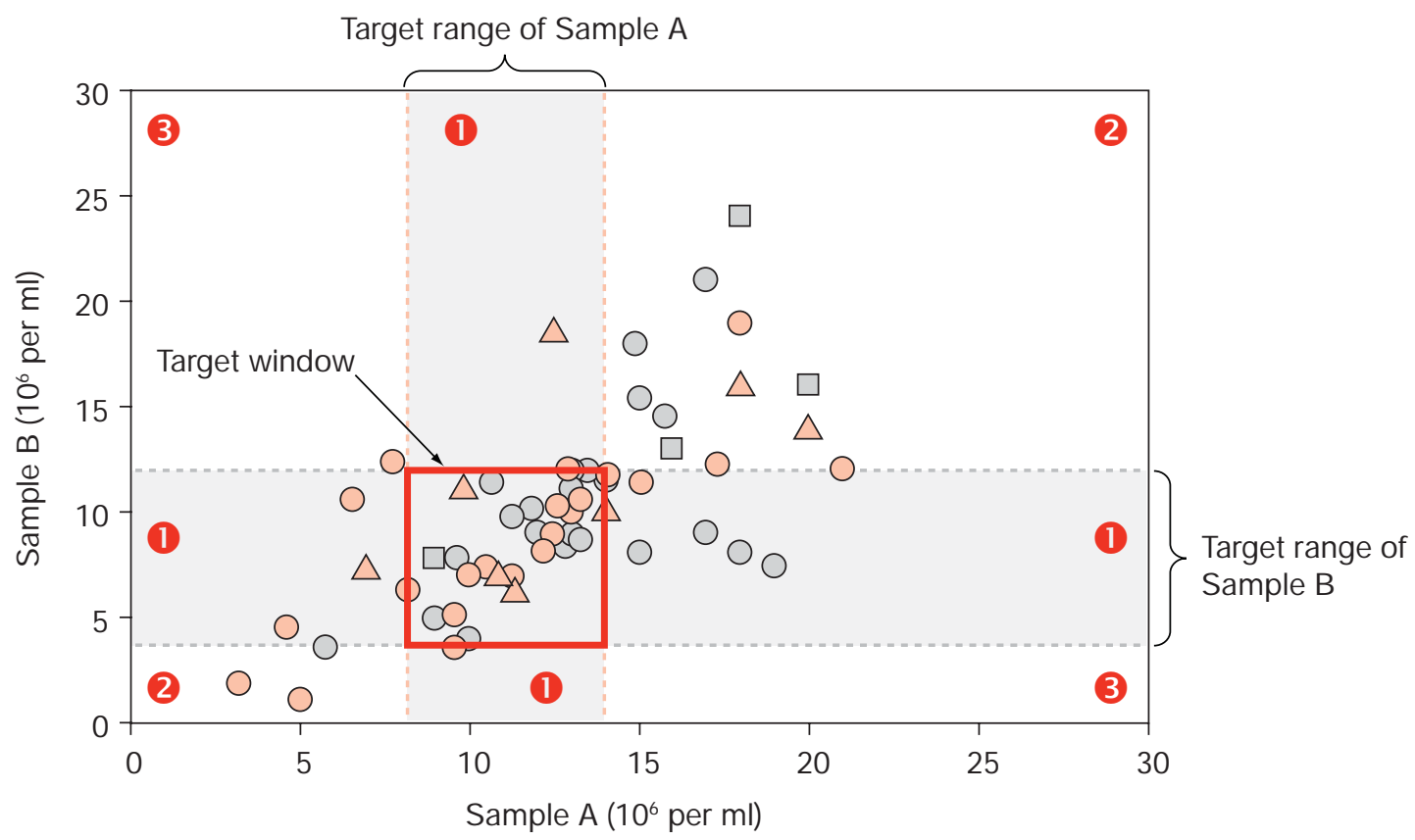

- Two-way analysis of variance. This technique is described in many statistical textbooks (e.g. Armitage et al., 2002) and is available in computer programs, together with statistical tests for the significance of differences between technicians. As with the paired comparison above, differences between all technicians' estimates should be zero. Thus, the differences from the average value are computed for every sample for each technician, and the mean and standard deviation of these differences are computed for each technician. Bias is indicated for technicians for whom the absolute value of the difference is more than 3 standard errors from the mean difference.

A formal statistical test for differences between technicians is based on the F-test from the two-way analysis of variance table, which can be obtained directly from most statistics computer programs. The error root mean square $(\hat{\sigma})$ is the square root of the residual, or error, mean square from the analysis of variance table.

Mean differences greater than about 2.5 standard errors are unlikely to result from chance variation alone $(<1.2 \%)$. Whether the differences between technicians are significant or not, it is necessary to review the technicians' means or mean differences to identify which are greater than expected. Not all computer packages pro- 
vide the standard error of the differences between technicians, which may have to be computed separately. Substantial differences between technicians should prompt a review of all procedures to identify how consistency can be improved.

The worked example in Box 7.6 illustrates how to compute the standard error of the differences among technicians in sperm concentration directly, and assess whether these are greater than would be expected from chance variation alone. When performing computations directly from the observations, a sufficient number of decimal places must be kept to avoid rounding errors.

7.10.2 Monitoring monthly means

While the primary IQC procedures are based on assessment of differences among and within technicians, additional information may be obtained by monitoring trends in results of semen analysis.

The mean values of each variable for all the patients examined over a certain period (e.g. monthly) can be plotted on an $X_{b a r}$ chart, with warning and action limits 2 and 3 standard errors either side of the mean. The standard error can be estimated from the standard deviation of the original observations divided by the square root of the number of semen analyses in each interval, or directly from the observed distribution of the mean. The control limits should be determined using at least 6 months' observations, and should be revised regularly. There should be at least 20 results for each mean; a small laboratory may have to pool results from more than 1 month. Refinements to the method include monitoring monthly means of patients with normal values and the use of cumulative sum (CUSUM) charts for the rapid detection of any systematic departures from the mean (Barnett, 1979).

Deviations from the expected values may reflect different client characteristics (time-dependent changes in the men being analysed; a change in the number of repeat tests on the same men; changes in the pattern of referral of men with different types of infertility) or technical factors (changes in technicians, laboratory supplies, seasonal temperature variations, etc.).

\subsection{External quality control and quality assurance}

External quality control (EQC) is an integral part of the complete $\mathrm{QC}$ process (Cekan et al., 1995) that monitors assay results, while external quality assurance (EQA) monitors all laboratory procedures relating to collecting and reporting data to ensure that laboratory processes are under control. EQC allows a laboratory to compare its results with those of others. It permits different methods to be evaluated and compared on a scale not possible in a single laboratory.

EQC and IQC are complementary processes. EQC may reveal problems with accuracy that may not be apparent from IQC if control samples are not adequately masked or selected. EQC has the advantage that it allows a laboratory to monitor the accuracy and stability of its methods (Plaut \& Westgard, 2002). However, as EQC samples are clearly of external origin, they are liable to be handled in a special way; this should be guarded against so that they are processed as far as possible in the same way as routine samples. 
Box 7.6 Assessing systematic differences among technicians

The table below shows sperm concentrations estimated by three technicians on five QC samples. Sperm concentration $\left(10^{6}\right.$ per $\left.\mathrm{ml}\right)$

\begin{tabular}{|l|c|c|c|c|c|}
\hline Sample & 1 & 2 & 3 & 4 & 5 \\
\hline Technician A & 108 & 45 & 100 & 50 & 92 \\
\hline Technician B & 103 & 47 & 102 & 50 & 96 \\
\hline Technician C & 104 & 46 & 89 & 41 & 88 \\
\hline Sample mean & 105 & 46 & 97 & 47 & 92 \\
\hline
\end{tabular}

The differences from the sample mean $\left(\mathrm{d}_{\mathrm{ij}}\right)$ are computed by subtracting the semen sample mean from each observation:

\begin{tabular}{|l|c|c|c|c|c|}
\hline Sample & 1 & 2 & 3 & 4 & 5 \\
\hline Technician A & 3.0 & -1.0 & 3.0 & 3.0 & 0.0 \\
\hline Technician B & -2.0 & 1.0 & 5.0 & 3.0 & 4.0 \\
\hline Technician C & -1.0 & 0.0 & -8.0 & -6.0 & -4.0 \\
\hline
\end{tabular}

The mean, $m_{j}=\Sigma_{i} d_{i j} / n$, and standard deviation, $s_{j}=\sqrt{ }\left(\Sigma_{i} d_{i j}{ }^{2} /(n-1)\right)$, of these differences are computed for each technician, where $n$ is the number of semen samples.

\begin{tabular}{|l|c|c|c|}
\hline & Mean $\left(\mathrm{m}_{\mathrm{j}}\right)$ & $\mathrm{SD}\left(\mathrm{s}_{\mathrm{j}}\right)$ & Mean/standard error $\left(\mathrm{m}_{\mathrm{j}} / \mathrm{se}\left(\mathrm{m}_{\mathrm{j}}\right)\right)$ \\
\hline Technician A & 1.600 & 1.949 & 1.836 \\
\hline Technician B & 2.200 & 2.775 & 1.773 \\
\hline Technician C & -3.800 & 3.347 & -2.539 \\
\hline
\end{tabular}

For technician $C$, the mean difference from the sample mean is $-3.8 \times 10^{6}$ per $\mathrm{ml}$, or $5.7(-3.8-(1.6+2.2) / 2) \times 10^{6}$ per $\mathrm{ml}$ less than the average of the other two technicians. To assess whether the degree of underestimation is compatible with chance variation, the error root mean square, $\hat{\sigma}=\sqrt{ }\left(\Sigma_{\mathrm{j}} \mathrm{s}_{\mathrm{j}}^{2} /(\mathrm{t}-1)\right)$, where $\mathrm{t}$ is the number of technicians, is computed from the standard deviations of the technicians' differences. In this example, $\hat{\sigma}=3.369 \times 10^{6} \mathrm{per} \mathrm{ml}$. The standard error of each technician's mean difference is given by se $\left(m_{j}\right)=\hat{\sigma} \sqrt{ }((1-1 / t) / n)$, or $1.230 \times 10^{6}$ per ml. The absolute value of technician C's mean difference $\left(3.8 \times 10^{6}\right.$ per $\left.\mathrm{ml}\right)$ is greater than 3 standard errors, and is therefore significantly different from the expected value of zero (assuming no systematic differences between the technicians).

A formal statistical test of differences between technicians is based on the F-test from the twoway analysis of variance for technicians and QC samples. The analysis of variance table, using the above sperm concentrations, is given below.

\begin{tabular}{|l|r|c|r|r|r|}
\hline Source & $\begin{array}{c}\text { Sum of } \\
\text { squares }\end{array}$ & $\begin{array}{c}\text { Degrees of } \\
\text { freedom }\end{array}$ & $\begin{array}{c}\text { Mean } \\
\text { square }\end{array}$ & F-ratio & P-value \\
\hline QC samples & 9807.6 & 4 & 2451.90 & 216.03 & $<0.001$ \\
\hline Technicians & 109.2 & 2 & 54.60 & 4.81 & 0.042 \\
\hline Error & 90.8 & 8 & 11.35 & & \\
\hline Total & 10007.6 & 14 & & & \\
\hline
\end{tabular}

The error root mean square is $\sqrt{ } 11.35=3.369 \times 10^{6}$ per $\mathrm{ml}$, the same as that obtained above. As expected, the differences between $Q C$ samples are very large $(P<0.001)$ since they are taken from different fresh semen samples. The $F$-test for differences between technicians ( $F=4.81$ with 2 and 8 degrees of freedom, $P=0.042$ ) is significant at the 0.05 level and suggests that these differences are greater than would be expected from random variation alone. 


\section{Box 7.7 Main features of IQC procedures}

\begin{tabular}{llll} 
Procedure & Errors detected & QC material & No. of technicians \\
\hline $\mathrm{X}_{\text {bar }}$ chart & $\begin{array}{l}\text { bias, overall variability, } \\
\text { accuracy }\end{array}$ & stored & individual, several \\
S chart & bias/precision & stored/fresh & several \\
Two-way ANOVA & bias/precision & stored/fresh & several \\
Bland-Altman & bias/precision & stored/fresh & two \\
Paired tests & bias/precision & stored/fresh & two \\
Youden plots & bias/precision & stored/fresh & several
\end{tabular}

EQC encompasses peer comparison and proficiency testing programmes in which specimens presumed to be identical are sent to all participating laboratories for analysis (Cembrowski \& Carey, 1989). Laboratories submit their results to a central facility where the data are examined for outliers, and means and standard deviations are calculated to characterize the performance of the participating laboratories. A list of national EQC programmes for semen analysis is given in Appendix 8 .

7.11.1 Assessment of EQC results

EQC schemes provide laboratories with information on both their results and those from other participating laboratories. It should be ascertained whether specified target values were obtained from accurate measurement, from multiple haemocytometer counts of sperm concentration, from computer-aided analysis of sperm motility, and if results obtained from a group of well-controlled reference laboratories or are trimmed means of all participating centres. Results are often presented graphically, such as in a bar chart. If the same EQC sample is used on several occasions, the bias and variability for the laboratory's results on this sample will also be reported.

When two samples are provided for analysis, a Youden plot is often constructed in which the values for each sample are plotted on the $x$ and $y$ axes (see Fig. 7.4). The extent to which centres differ in their assessment is clearly seen from the scatter and distribution of the plotted values. Additional data can be visualized, for example, by using different symbols or colours to indicate the use of different methods (counting chambers, stains or assessment criteria) or different centres.

When more than two samples are distributed, various aspects of bias (the difference from the designated value) may be given. These include:

- the bias index score (BIS): bias divided by a chosen coefficient of variation $\times 100$, which can be positive or negative;

- the variance index score (VIS): this is similar to the BIS but is always positive;

- the mean running BIS or VIS scores (MRBIS, MRVIS), which help ascertain trends. 
A low MRBIS and low MRVIS indicate that results are close to designated values; a low MRBIS but high MRVIS could indicate random error; and a high MRBIS and high MRVIS indicate systematic errors. Results reported as successful/unsuccessful or as ranks are useful for laboratory inspection and certification.

A simple way to monitor performance is to plot the laboratory's results (on the $y$-axis) against the target value (on the $x$-axis) for each parameter. This shows clearly how close to the line of identity the laboratory's values fall. Alternatively, differences from the target values can be shown on a Bland-Altman plot (see Fig. 7.3).

\subsubsection{Responses to out-of-control results}

The essential information derived from EQC programmes relates to the bias or accuracy of laboratories and laboratory methods. The desired outcome is for laboratories to maintain or improve the accuracy of their methods (Plaut \& Westgard, 2002). Laboratories with results that are persistently higher or lower than the assigned value or mean of the EQC scheme need to reappraise their methods. A wide variation in $E Q C$ results is usually associated with wide variation in IQC results and indicates inconsistencies in the assessment procedures from sample to sample. Technical procedures should be carefully reassessed to ensure that they conform to the recommendations in this manual.

Appropriate actions include those discussed for IQC (see Section 7.9.3) with retraining and retesting. Tables 7.2-7.5 also indicate potential sources of variation in sperm analysis and proposed solutions. Exchange of scientific staff between laboratories is often helpful and the training of technicians in laboratories with good EQC results can be beneficial. A consultant from a laboratory with good $E Q C$ results will often be able to see where methods could be changed to improve reproducibility.

\subsection{Frequency and priority of quality control}

The QC samples should be analysed routinely. The frequency of analysis may be determined by national or local recommendations or mandated by laboratory licensing laws or accreditation agencies. Some regulations require that QC samples are analysed each day that patient sperm concentrations are assessed; otherwise between $1 \%$ and $5 \%$ of samples should be for IQC.

QC samples should be used:

- to monitor newly employed and existing staff;

- whenever new laboratory equipment, supplies, procedures, or batches of IQC samples are introduced.

Box 7.8 contains a general guide to scheduling of QC; in practice, the schedule will depend on the workload in the laboratory. Box 7.9 indicates the priority of the different QC protocols; some procedures may not be feasible for laboratories with limited funding. 


\section{Box 7.8 Time schedule for quality control}

$\begin{array}{ll}\text { At all times } & \text { surveillance and correlation of results within samples } \\ \text { Weekly/monthly } & \text { analysis of replicate measurements by different technicians } \\ \text { Monthly/quarterly } & \text { analysis of mean results } \\ \text { Quarterly/6-monthly } & \text { participation in EQC } \\ \text { 6-monthly/yearly } & \text { calibration of pipettes, counting chambers, other equipment }\end{array}$

\section{Box 7.9 Summary of QC tests}

$\begin{array}{clclll}\text { Parameter } & \text { Material } & \begin{array}{c}\text { Target } \\ \text { value }\end{array} & \begin{array}{c}\text { Accuracy, } \\ \text { bias }\end{array} & \text { Precision } & \begin{array}{c}\text { Priority } \\ (1>2>3)\end{array} \\ \text { Concentration } & \text { IQC fresh } & \text { No } & & \text { S chart, 2-way ANOVA } & 1 \\ & \text { IQC stored } & \text { Yes } & X_{\text {bar }} \text { chart } & \text { S chart } & 3 \\ \text { Morphology } & \text { EQC } & \text { Yes } & X_{\text {bar }} \text { chart } & \text { S chart } & 2 \\ & \text { IQC fresh } & \text { No } & & \text { S chart, 2-way ANOVA } & 1 \\ \text { Motility } & \text { IQC stored } & \text { Yes } & X_{\text {bar }} \text { chart } & \text { S chart } & 3 \\ & \text { EQC } & \text { Yes } & X_{\text {bar }} \text { chart } & \text { S chart } & 2 \\ \text { Vitality } & \text { IQC fresh } & \text { No } & & \text { S chart, 2-way ANOVA } & 1 \\ & \text { IQC stored } & \text { Yes } & X_{\text {bar }} \text { chart } & \text { S chart } & 3 \\ & \text { EQC } & \text { Yes } & X_{\text {bar }} \text { chart } & \text { S chart } & 2 \\ & \text { IQC fresh } & \text { No } & & \text { S chart, 2-way ANOVA } & 1 \\ & \text { IQC stored } & \text { Yes } & X_{\text {bar }} \text { chart } & \text { S chart } & 3 \\ & \text { EQC } & \text { Yes } & X_{\text {bar }} \text { chart } & \text { S chart } & 2\end{array}$

\subsection{Training}

A similar approach to QC can be used when technicians are being trained, new assays introduced, or modifications to existing methods assessed. Technician training should include awareness of the approaches outlined below.

7.13.1 Practical hints when experiencing difficulty assessing sperm concentration

- Review the mixing and dilution procedures, chamber grids and calculations.

- Read the samples within 10-15 minutes of loading the chamber, after which evaporation has noticeable effects on the position of spermatozoa within the chamber.

- Two technicians should work together, using a bridge microscope or microscope equipped with a video camera and a TV screen, comparing dilution, loading and counting procedures. They should count the same loaded chamber, comparing values for rows or grids, to find the source of discrepancies.

- Use a bridge microscope in a counting and training session, or examine spermatozoa in the grid ocular, to decide whether individual spermatozoa are considered on a line and should be eligible for inclusion in the count.

- Review Table 7.2. 
Table 7.2 Sources of variation (error) in assessing sperm concentration and proposed solutions

\begin{tabular}{|c|c|c|}
\hline Procedure & Prevention & Control \\
\hline $\begin{array}{l}\text { Incomplete mixing of semen samples before making } \\
\text { dilution }\end{array}$ & Training, SOP & Replicate dilutions \\
\hline $\begin{array}{l}\text { Dilution errors (e.g. assuming a 1:20 dilution is } 1+20 \text {, } \\
\text { when it is in fact } 1+19 \text { ) }\end{array}$ & Training, SOP & IQC \\
\hline $\begin{array}{l}\text { Pipetting device out of calibration (e.g. pipette is set to } \\
100 \mu \text { l but actually delivers } 95 \mu \text { or } 110 \mu l \text { ) }\end{array}$ & $\begin{array}{l}\text { Equipment } \\
\text { maintenance, SOP }\end{array}$ & $\begin{array}{l}\text { Replicate dilutions, } \\
\text { IQC, EQC }\end{array}$ \\
\hline $\begin{array}{l}\text { Using an inappropriate pipette (e.g. an air- rather than a } \\
\text { positive-displacement pipette) }\end{array}$ & Training, SOP & $\begin{array}{l}\text { Replicate dilutions, } \\
\text { IQC, EQC }\end{array}$ \\
\hline $\begin{array}{l}\text { Using a low volume for dilution, which carries a high } \\
\text { risk of unrepresentative sampling }\end{array}$ & Training, SOP & $\begin{array}{l}\text { Replicate dilutions, } \\
\text { IQC, EQC }\end{array}$ \\
\hline $\begin{array}{l}\text { Failure to wipe the residual semen from the outside of } \\
\text { the pipette tip before dispensing it into the diluent }\end{array}$ & Training, SOP & IQC \\
\hline Chamber not clean and dry & Training, SOP & $\begin{array}{l}\text { Replicate } \\
\text { assessments }\end{array}$ \\
\hline $\begin{array}{l}\text { Chamber incorrectly assembled or loaded (e.g. dirt } \\
\text { particles on the pillars may alter chamber height) }\end{array}$ & Training, SOP & $\begin{array}{l}\text { Replicate } \\
\text { assessments }\end{array}$ \\
\hline $\begin{array}{l}\text { Excessive time lag between mixing semen and remov- } \\
\text { ing aliquot for dilution (spermatozoa in semen start to } \\
\text { settle immediately) }\end{array}$ & Training, SOP & $\begin{array}{l}\text { Replicate dilutions } \\
\text { and assessments }\end{array}$ \\
\hline $\begin{array}{l}\text { Excessive time lag between vortexing the dilution and } \\
\text { loading chamber (diluted spermatozoa start to settle } \\
\text { immediately) }\end{array}$ & Training, SOP & $\begin{array}{l}\text { Replicate dilutions } \\
\text { and assessments }\end{array}$ \\
\hline $\begin{array}{l}\text { Microscope not properly cleaned or aligned. Incorrect } \\
\text { magnification }\end{array}$ & $\begin{array}{l}\text { Training, SOP, } \\
\text { equipment } \\
\text { maintenance }\end{array}$ & IQC and EQC \\
\hline $\begin{array}{l}\text { Not waiting long enough after loading chamber before } \\
\text { analysis (insufficient time for sedimentation) }\end{array}$ & Training, SOP & $\begin{array}{l}\text { Replicate assess- } \\
\text { ments, IQC, EQC }\end{array}$ \\
\hline $\begin{array}{l}\text { Haemocytometer chamber not horizontal during sperm } \\
\text { settling, or chamber not kept in a humidified environ- } \\
\text { ment during settling }\end{array}$ & Training, SOP & $\begin{array}{l}\text { Replicate assess- } \\
\text { ments, IQC, EQC }\end{array}$ \\
\hline $\begin{array}{l}\text { Misidentification of spermatozoa (e.g. counting debris } \\
\text { as spermatozoa or missing hard-to-recognize sperma- } \\
\text { tozoa) }\end{array}$ & Training, SOP & IQC, EQC \\
\hline $\begin{array}{l}\text { Assessing too few or too many rows on grid (i.e. incor- } \\
\text { rect calculations); stopping in the middle of a row }\end{array}$ & Training, SOP & IQC, EQC \\
\hline $\begin{array}{l}\text { Counting too few spermatozoa, leading to high sam- } \\
\text { pling error }\end{array}$ & Training, SOP & IQC, EQC \\
\hline $\begin{array}{l}\text { Inconsistently scoring spermatozoa on the counting } \\
\text { box lines (e.g. overestimating concentration if sperma- } \\
\text { tozoa are scored on top, bottom, left and right borders) }\end{array}$ & Training, SOP & IQC, EQC \\
\hline Malfunction of multikey counter & $\begin{array}{l}\text { Equipment } \\
\text { maintenance }\end{array}$ & IQC. EQC \\
\hline $\begin{array}{l}\text { Mathematical error in calculating, or correcting for } \\
\text { dilution }\end{array}$ & Training, SOP & IQC, EQC \\
\hline $\begin{array}{l}\text { Use of capillary-filled chamber (unequal distribution of } \\
\text { spermatozoa during filling) }\end{array}$ & Training, SOP & IQC, EQC \\
\hline
\end{tabular}


7.13.2 Practical hints when experiencing difficulty assessing sperm morphology

- Adhere to the guidelines in this manual: study the micrographs and the relevant commentary for each spermatozoon.

- Pay particular attention to spermatozoa with borderline morphology; these should be classified as abnormal.

- Conduct a scoring and training session using a bridge microscope or microscope equipped with a video camera and a TV screen.

- Review Table 7.3.

Table 7.3 Sources of variation (error) in assessing sperm morphology and proposed solutions

\begin{tabular}{|l|l|l|}
\hline Procedure & Prevention & Control \\
\hline $\begin{array}{l}\text { Microscope not properly cleaned or aligned. Incorrect } \\
\text { magnification }\end{array}$ & $\begin{array}{l}\text { Training, SOP, } \\
\text { equipment } \\
\text { maintenance }\end{array}$ & IQC, EQC \\
\hline Inadequate training before performing analysis & Training & IQC, EQC \\
\hline Subjective techniques without clear guidelines & Training, SOP & IQC, EQC \\
\hline $\begin{array}{l}\text { Subtle influences of peers on classification systems } \\
\text { (may cause changes during analysis) }\end{array}$ & Training & IQC (control charts) \\
\hline Semen inadequately mixed when smear was prepared & Training, SOP & IQC \\
\hline Poor smear preparation (i.e. too thick or too thin) & Training, SOP & IQC \\
\hline $\begin{array}{l}\text { Poor staining technique (i.e. light, dark, or too much } \\
\text { background staining) }\end{array}$ & Training, SOP & IQC \\
\hline Assessing spermatozoa on edge of slide & Training, SOP & IQC \\
\hline $\begin{array}{l}\text { Attempting to score spermatozoa that are not flat, or } \\
\text { are overlapping other spermatozoa }\end{array}$ & Training, SOP & IQC \\
\hline $\begin{array}{l}\text { Not scoring all spermatozoa in area but selecting sper- } \\
\text { matozoa for assessment }\end{array}$ & Training, SOP & IQC \\
\hline Fading of stain over time (for stored IQC samples) & Training, SOP & IQC (control chart) \\
\hline $\begin{array}{l}\text { Errors in calculating percentages if not counted in } \\
\text { multiples of 100 }\end{array}$ & Training, SOP & IQC, EQC \\
\hline $\begin{array}{l}\text { Malfunction of multikey counter } \\
\text { Eaten }\end{array}$ & $\begin{array}{l}\text { Equipment } \\
\text { maintenance }\end{array}$ & IQC, EQC \\
\hline
\end{tabular}

7.13.3 Practical hints when experiencing difficulty assessing sperm motility

- Make the preparation immediately before assessing. Read only after any drifting has stopped to reduce bias in overall motility.

- Select the field randomly and do not deliberately select fields with high or low numbers of motile spermatozoa. One way to do this is to avoid looking through the oculars until a field has been selected. 
- Do not wait for motile spermatozoa to enter the field before starting to count.

- Analyse quickly; analyse only a small portion of the grid at one time, depending on sperm concentration.

- Spend less time examining one area of the grid, to avoid counting spermatozoa that swim into the area during analysis.

- Count progressive, non-progressive and immotile spermatozoa in two stages. If there are problems with the technique, reverse the order of analysis.

- Review Table 7.4.

Table 7.4 Sources of variation (error) in assessing sperm motility and proposed solutions

\begin{tabular}{|c|c|c|}
\hline Procedure & Prevention & Control \\
\hline Improper mixing of specimen before aliquot is removed & Training, SOP & $\begin{array}{l}\text { Replicate sampling } \\
\text { and assessment, } \\
\text { IQC }\end{array}$ \\
\hline $\begin{array}{l}\text { Waiting too long after slide is prepared before analysis } \\
\text { (spermatozoa quickly lose vigour) }\end{array}$ & Training, SOP & $\begin{array}{l}\text { Replicate sampling } \\
\text { and assessment, } \\
\text { IQC }\end{array}$ \\
\hline $\begin{array}{l}\text { Improper temperature of stage warmer (e.g. too high } \\
\text { temperature will kill spermatozoa) }\end{array}$ & $\begin{array}{l}\text { Training, SOP, } \\
\text { equipment } \\
\text { maintenance }\end{array}$ & IQC \\
\hline $\begin{array}{l}\text { Microscope not properly cleaned or aligned. Improper } \\
\text { magnification }\end{array}$ & $\begin{array}{l}\text { Training, SOP, } \\
\text { equipment } \\
\text { maintenance }\end{array}$ & IQC, EQC \\
\hline Lack of eyepiece grid for guidance & Equipment & IQC (control chart) \\
\hline $\begin{array}{l}\text { Analysing around the edges of the coverslip (the } \\
\text { spermatozoa die or become sluggish around the outer } \\
5 \mathrm{~mm} \text { of the coverslip) }\end{array}$ & Training, SOP & $\begin{array}{l}\text { Replicate } \\
\text { assessment, IQC }\end{array}$ \\
\hline $\begin{array}{l}\text { Making the assessment too slowly (other spermato- } \\
\text { zoa swim into the defined area during the assessment } \\
\text { period) }\end{array}$ & Training, SOP & IQC \\
\hline Malfunction of multikey counter & $\begin{array}{l}\text { Equipment } \\
\text { maintenance }\end{array}$ & IQC, EQC \\
\hline $\begin{array}{l}\text { Errors in calculating percentages if not counted in } \\
\text { multiples of } 100\end{array}$ & Training, SOP & IQC, EQC \\
\hline $\begin{array}{l}\text { Subjective bias (i.e. consistently too high } \% \text { motile or } \\
\text { too low } \% \text { motile) }\end{array}$ & Training, SOP & IQC, EQC \\
\hline $\begin{array}{l}\text { P reparative procedures that reduce motility (e.g. tem- } \\
\text { perature change, vigorous mixing, contamination with } \\
\text { toxins) }\end{array}$ & SOP & IQC \\
\hline $\begin{array}{l}\text { Non-random selection of fields for analysis. Delay in } \\
\text { analysis (e.g. waiting until motile spermatozoa swim } \\
\text { into the field or grid to begin analysis) }\end{array}$ & Training, SOP & IQC, EQC \\
\hline
\end{tabular}


7.13.4 Practical hints when experiencing difficulty assessing sperm vitality

- Pay particular attention to distinguishing between red (dead) and pink (alive) sperm heads (spermatozoa with faint pink head staining are assessed as alive). If the stain is limited to a part of the neck region, and the rest of the head area is unstained, this is considered a "leaky neck membrane", but not a sign of cell death and total membrane disintegration.

- Consider using the eosin-nigrosin method (see Section 2.6.1).

- Review Table 7.5.

Table 7.5 Sources of variation (error) in assessing sperm vitality and proposed solutions

\begin{tabular}{|l|l|l|}
\hline Procedure & Prevention & Control \\
\hline $\begin{array}{l}\text { Microscope not properly cleaned or aligned. Improper } \\
\text { magnification }\end{array}$ & $\begin{array}{l}\text { Training, SOP, } \\
\text { equipment } \\
\text { maintenance }\end{array}$ & IQC, EQC \\
\hline $\begin{array}{l}\text { Improper staining: some recipes give hypo-osmotic } \\
\text { conditions that kill spermatozoa }\end{array}$ & Training, SOP & $\begin{array}{l}\text { Comparison with } \\
\text { motility }\end{array}$ \\
\hline Waiting too long to stain & Training, SOP & $\begin{array}{l}\text { Comparison with } \\
\text { motility }\end{array}$ \\
\hline $\begin{array}{l}\text { Rehydration of dried smear, if not mounted directly, will } \\
\text { allow stain to leak into all spermatozoa }\end{array}$ & Training, SOP & $\begin{array}{l}\text { Comparison with } \\
\text { motility }\end{array}$ \\
\hline $\begin{array}{l}\text { Overestimation of dead spermatozoa (e.g. perceiving as } \\
\text { dead sperm heads with slight pink stain) }\end{array}$ & Training, SOP, & IQC, EQC \\
\hline $\begin{array}{l}\text { Assessing spermatozoa with pink staining restricted to } \\
\text { the neck as dead }\end{array}$ & Training, SOP & IQC, EQC \\
\hline
\end{tabular}


References 



\section{REFERENCES}

Abraham-Peskir JV et al. (2002). Response of midpiece vesicles on human sperm to osmotic stress. Human Reproduction, 17:375-382.

Abshagen K et al. (1998). Influence of sperm surface antibodies on spontaneous pregnancy rates. Fertility and Sterility, 70:355-356.

Agarwal A et al. (2004). Role of antioxidants in treatment of male infertility: an overview of the literature. Reproductive Biomedicine Online, 8:616-627.

Aitken RJ , Baker HW (1995). Seminal leukocytes: passengers, terrorists or good Samaritans? Human Reproduction, 10:1736-1739.

Aitken RJ , Clarkson J S (1987). Cellular basis of defective sperm function and its association with the genesis of reactive oxygen species by human spermatozoa. J ournal of Reproduction and Fertility, 81:459-469.

Aitken RJ , Clarkson J S (1988). Significance of reactive oxygen species and antioxidants in defining the efficacy of sperm preparation techniques. J ournal of Andrology, 9:367-376.

Aitken RJ , Elton RA (1986). Application of a Poisson-gamma model to study the influence of gamete concentration on sperm-oocyte fusion in the zona-free hamster egg penetration test. J ournal of Reproduction and Fertility, 78:733-739.

Aitken RJ , Krausz CG (2001). Oxidative stress, DNA damage and the Y chromosome. Reproduction, 122:497-506.

Aitken RJ , West KM (1990). Analysis of the relationship between reactive oxygen species production and leucocyte infiltration in fractions of human semen separated on Percoll gradients. International J ournal of Andrology, 13:433-451.

Aitken RJ et al. (1983). Methods for assessing the functional capacity of human spermatozoa; their role in the selection of patients for in-vitro fertilization. In: Beier H, Lindner H, eds, Fertilization of the human egg in vitro: Biological basis and clinical application. Berlin, Springer: 147-168.

Aitken RJ et al. (1992). Reactive oxygen species and human spermatozoa: analysis of the cellular mechanisms involved in luminol- and lucigenin-dependent chemiluminescence. J ournal of Cellular Physiology, 151:466- 477.

Aitken RJ et al. (1993). Analysis of the response of human spermatozoa to A23187 employing a novel technique for assessing the acrosome reaction. J ournal of Andrology, 14:132-141.

Aitken RJ et al. (2003). Oxidative stress in the male germ line and its role in the aetiology of male infertility and genetic disease. Reproductive Biomedicine Online, 7:65-70.

Aitken RJ et al. (2004). Shedding light on chemiluminescence: the application of chemiluminescence in diagnostic andrology. J ournal of Andrology, 25:455-465.

Alvarez C et al. (2003). Biological variation of seminal parameters in healthy subjects. Human Reproduction, 18:2082-2088.

Alvarez J G et al. (1987). Spontaneous lipid peroxidation and production of hydrogen peroxide and superoxide in human spermatozoa. Superoxide dismutase as major enzyme protectant against oxygen toxicity. J ournal of Andrology, 8:338-348.

Andersen AG et al. (2000). High frequency of sub-optimal semen quality in an unselected population of young men. Human Reproduction, 15:366-372.

Armitage P et al. (2002). Statistical methods in medical research. Oxford, Blackwell Science. 
Auger J , Eustache F (2000). Standardisation de la classification morphologique des spermatozoides humains selon la méthode de David modifieé. Andrologia, 10:358-373.

Auger J et al. (1995). Decline in semen quality among fertile men in Paris during the past 20 years. New England J ournal of Medicine, 332:281-285.

Auger J et al. (2001). Sperm morphological defects related to environment, lifestyle and medical history of 1001 male partners of pregnant women from four European cities. Human Reproduction, 16:2710-2717.

Aziz N et al. (1996). The sperm deformity index: a reliable predictor of the outcome of oocyte fertilization in vitro. Fertility and Sterility, 66:1000-1008.

Aziz N et al. (2004). Novel association between sperm reactive oxygen species production, sperm morphological defects, and the sperm deformity index. Fertility and Sterility, 81:349-354.

Baker HW, Kovacs GT (1985). Spontaneous improvement in semen quality: regression towards the mean. International J ournal of Andrology, 8:421-426.

Barnett RN (1979). Clinical laboratory statistics, 2nd ed. Boston, Little, Brown.

B arratt CLR et al. (1992). The poor prognostic value of low to moderate levels of sperm surface-bound antibodies. Human Reproduction, 7:95-98.

Barratt CLR et al. (1993). Prognostic significance of computerized motility analysis for in-vivo fertility. Fertility and Sterility, 60:520-525.

Bedford J M (1977). Sperm/egg interaction: the specificity of human spermatozoa. Anatomical Record, 188:477-487.

Behre HM et al. (2000). Diagnosis of male infertility and hypogonadism. In: Nieschlag $E, B$ ehre $H M$, eds. Andrology, male reproductive health and dysfunction. Berlin, Springer: 92.

Berman NG et al. (1996). Methodological issues in the analysis of human sperm concentration data. J ournal of Andrology, 17:68-73.

Biggers J D et al. (1971). The culture of mouse embryos in vitro. In: Daniel J C, ed. Methods in mammalian embryology. San Francicso, WH Freeman: 86-116.

Björndahl L, Barratt CL (2005). Semen analysis: setting standards for the measurement of sperm numbers. J ournal of Andrology, 26:11.

Björndahl L, Kvist U (2003). Sequence of ejaculation affects the spermatozoon as a carrier and its message. Reproductive Biomedicine Online, 7:440-448.

Björndahl L et al. (2003). Evaluation of the one-step eosin-nigrosin staining technique for human sperm vitality assessment. Human Reproduction, 18:813-816.

Björndahl L et al. (2004). Why the WHO recommendations for eosin-nigrosin staining techniques for human sperm vitality assessment must change. J ournal of Andrology, 25:671-678.

Björndahl L et al. (2005). Contamination by seminal plasma factors during sperm selection. J ournal of Andrology, 26:170-173.

Bland J M, Altman DG (1986). Statistical methods for assessing agreement between two methods of clinical measurement. Lancet, 1:307-310.

Bohring C, Krause W (1999). The intra- and inter-assay variation of the indirect mixed antiglobulin reaction test: is a quality control suitable? Human Reproduction, 14:1802-1805. 
Bonde J P et al. (1998). Relation between semen quality and fertility: a population-based study of 430 first-pregnancy planners. Lancet, 352:1172-1177.

Boomsma CM et al. (2004). Semen preparation techniques for intrauterine insemination. Cochrane Database of Systematic Reviews, CD004507.

Bourne $\mathrm{H}$ et al. (1995). Sperm preparation for intracytoplasmic injection: methods and relationship to fertilization results. Reproduction, Fertility, Development, 7:177-183.

Brazil C et al. (2004a). Standardized methods for semen evaluation in a multicenter research study. J ournal of Andrology, 25:635-644.

B razil C et al. (2004b). Quality control of laboratory methods for semen evaluation in a multicenter research study. J ournal of Andrology, 25:645-656.

Bronson RA et al. (1982). Detection of sperm specific antibodies on the spermatozoa surface by immunobead binding. Archives of Andrology, 9:61.

Bronson RA et al. (1984). Sperm antibodies: their role in infertility. Fertility and Sterility, $42: 171-183$.

Bunge RGT, Sherman J K (1953). Fertilizing capacity of frozen human spermatozoa. Nature, 172:767-768.

Bunge RG et al. (1954). Clinical use of frozen semen: report of four cases. Fertility and Sterility, 5:520-529.

Burkman LJ et al. (1988). The hemizona assay (HZA): development of a diagnostic test for the binding of human spermatozoa to human hemizona pellucida to predict fertilization potential. Fertility and Sterility, 49:688-697.

Canale D et al. (1994). Inter- and intra-individual variability of sperm morphology after selection with three different techniques: layering, swimup from pellet and Percoll. J ournal of Endocrinological Investigation, 17:729-732.

Carey RG, Lloyd RC (1995). Measuring quality improvement in healthcare: a guide to statistical process control applications. New York, Quality Resources.

Carlsen E et al. (2004). Effects of ejaculatory frequency and season on variations in semen quality. Fertility and Sterility, 82:358-366.

Carreras A et al. (1992). Sperm plasma membrane integrity measurement: a combined method. Andrologia, 24:335-340.

Castilla J A et al. (2006). Influence of analytical and biological variation on the clinical interpretation of seminal parameters. Human Reproduction, 21:847-851.

Cekan SZ et al. (1995). Principles of external quality assessment: a laboratory manual. Geneva, World Health Organization.

Cembrowski GS, Carey RN (1989). Laboratory quality management. Chicago, ASCP Press.

Chantler E, Abraham-Peskir J V (2004). Significance of midpiece vesicles and functional integrity of the membranes of human spermatozoa after osmotic stress. Andrologia, 36:87-93.

Chemes HE, Rawe YV (2003). Sperm pathology: a step beyond descriptive morphology. Origin, characterization and fertility potential of abnormal sperm phenotypes in infertile men. Human Reproduction Update, 9:405-428.

Chiu WW, Chamley LW (2004). Clinical associations and mechanisms of action of antisperm antibodies. Fertility and Sterility, 82:529-535. 
Chohan KR et al. (2006). Comparison of chromatin assays for DNA fragmentation evaluation in human sperm. J ournal of Andrology, 27:53-59.

Christensen $\mathrm{P}$ et al. (2005). Discrepancies in the determination of sperm concentration using Bürker-Türk, Thoma and Makler counting chambers. Theriogenology, 63:992-1003.

Clarke GN (1999). Sperm cryopreservation: is there a significant risk of crosscontamination? Human Reproduction 14:2941-2943.

Clarke GN et al. (1982). Immunoglobulin class of sperm-bound antibodies in semen. In: Bratanov K, ed. Immunology of reproduction. Sofia, Bulgarian Academy of Sciences Press: $482-485$.

Clarke GN et al. (1985). Detection of sperm antibodies in semen using the Immunobead test: a survey of 813 consecutive patients. American J ournal of Reproductive Immunology and Microbiology, 7:118-123.

Clarke GN et al. (1997). Artificial insemination and in-vitro fertilization using donor spermatozoa: a report on 15 years of experience. Human Reproduction, 12:722-726.

Clarke GN et al. (2003). Improved sperm cryopreservation using cold cryoprotectant. Reproduction, Fertility, Development, 15:377-381.

Clarke GN et al. (2006). Recovery of human sperm motility and ability to interact with the human zona pellucida after more than 28 years of storage in liquid nitrogen. Fertility and Sterility, 86:721-722.

Coetzee K et al. (1998). Predictive value of normal sperm morphology: a structured literature review. Human Reproduction Update, 4:73-82.

Coetzee K et al. (1999a). Repeatability and variance analysis on multiple computer-assisted (IVOS ) sperm morphology readings. Andrologia, 31:163-168.

Coetzee K et al. (1999b). Assessment of interlaboratory and intralaboratory sperm morphology readings with the use of a Hamilton Thorne Research integrated visual optical system semen analyzer. Fertility and Sterility, 71:80-84.

Coetzee K et al. (1999c). Clinical value of using an automated sperm morphology analyzer (IVOS ). Fertility and Sterility, 71:222-225.

Cooper TG (2005). Cytoplasmic droplets: the good, the bad or just confusing? Human Reproduction, 20:9-11.

Cooper TG, Yeung CH (1998). A flow cytometric technique using peanut agglutinin for evaluating acrosomal loss from human spermatozoa. J ournal of Andrology, 19:542-550.

Cooper TG, Yeung CH (2006). Computer-aided evaluation of assessment of "grade a" spermatozoa by experienced technicians. Fertility and Sterility, 85:220-224.

Cooper TG et al. (1990a). The influence of inflammation of the human male genital tract on secretion of the seminal markers $\alpha$-glucosidase, glycerophosphocholine, carnitine, fructose and citric acid. International J ournal of Andrology, 13:329-335.

Cooper TG et al. (1990b). Improvement in the assessment of human epididymal function by the use of inhibitors in the assay of $\alpha$-glucosidase in seminal plasma. International J ournal of Andrology, 13:297-305.

Cooper TG et al. (1991). Variations in semen parameters from fathers. Human Reproduction, 6:859-866. 
Cooper TG et al. (1993). Effects of multiple ejaculations after extended periods of sexual abstinence on total, motile and normal sperm numbers, as well as accessory gland secretions, from healthy normal and oligozoospermic men. Human Reproduction, 8:1251-1258.

Cooper TG et al. (1999). Experience with external quality control in spermatology. Human Reproduction, 14:765-769.

Cooper TG et al. (2002). Semen analysis and external quality control schemes for semen analysis need global standardization. International J ournal of Andrology, 25:306-311.

Cooper TG et al. (2004). Cytoplasmic droplets are normal structures of human spermatozoa but are not well preserved by routine procedures for assessing sperm morphology. Human Reproduction, 19:2283-2288.

Cooper TG et al. (2005). Changes in osmolality during liquefaction of human semen. International J ournal of Andrology, 28:58-60.

Cooper TG et al. (2006). Azoospermia: virtual reality or possible to quantify? J ournal of Andrology, 27:483-490.

Cooper TG et al. (2007). Ejaculate volume is seriously underestimated when semen is pipetted or decanted into cylinders from the collection vessel. J ournal of Andrology, 28:1-4.

Cooper TG et al. (2010). World Health Organization reference values for human semen characteristics. Human Reproduction Update, 16:231-245.

Corea M et al. (2005). The diagnosis of azoospermia depends on the force of centrifugation. Fertility and Sterility, 83:920-922.

Correa-Perez J R et al. (2004). Clinical management of men producing ejaculates characterized by high levels of dead sperm and altered seminal plasma factors cons istent with epididymal necrospermia. Fertility and Sterility, 81:1148-1150.

Couture M et al. (1976). Improved staining method for differentiating immature germ cells from white blood cells in human seminal fluid. Andrologia, 8:61-66.

Cross NL (1995). Methods for evaluating the acrosomal status of human sperm. In: Fenichel P, Parinaud J, eds. Human sperm acrosome reaction. Paris, J ohn Libbey Eurotext (Colloques INSERM ): 277-285.

Cross NL et al. (1986). Two simple methods for detecting acrosome-reacted human sperm. Gamete Research, 15:213-226.

Dadoune J P et al. (1988). Correlation between defects in chromatin condensation of human spermatozoa stained by aniline blue and semen characteristics. Andrologia, 20:211-217.

Daudin M et al. (2000). Congenital bilateral absence of the vas deferens: clinical characteristics, biological parameters, cystic fibrosis transmembrane conductance regulator gene mutations, and implications for genetic counseling. Fertility and Sterility, 74:1164-1174.

David G et al. (1975). Anomalies morphologiques du spermatozoide humain. I. Propositions pour un système de classification. J ournal de Gynécologie, Obstétrique et Biologie de la Réproduction, 4(Suppl. 1):17-36.

David G et al. (1980). The success of A.I.D. and semen characteristics: study of 1489 cycles and 192 ejaculates. International J ournal of Andrology, 3:613-619. 
Davis RO, Katz DF (1992). Standardization and comparability of CASA instruments. J ournal of Andrology, 13:81-86.

De J onge C (2000). Commentary: forging a partnership between total quality management and the andrology laboratory. J ournal of Andrology, 21:203-205.

De J onge $C$ et al. (2004). Influence of the abstinence period on human sperm quality. Fertility and Sterility, 82:57-65.

de la Taille A et al. (1998). Correlation of genitourinary abnormalities, spermiogram and CFTR genotype in patients with bilateral agenesis of the vas deferens. Progress in Urology, 8:370-376.

Devillard F et al. (2002). Polyploidy in large-headed sperm: FISH study of three cases. Human Reproduction, 17:1292-1298.

Donnelly ET et al. (1998). In-vitro fertilization and pregnancy rates: the influence of sperm motility and morphology on IVF outcome. Fertility and Sterility, 70:305-314.

Douglas-Hamilton DH et al. (2005a). Particle distribution in low-volume capillary-loaded chambers. J ournal of Andrology, 26:107-114.

Douglas-Hamilton DH et al. (2005b). Capillary-loaded particle fluid dynamics: effect on estimation of sperm concentration. J ournal of Andrology, 26:115-122.

Drobnis EZ et al. (1988). Hamster sperm penetration of the zona pellucida: kinematic analysis and mechanical implications. Developmental Biology, 130:311-323.

Eggert-Kruse W et al. (1989). Prognostic value of in-vitro sperm penetration into hormonally standardized human cervical mucus. Fertility and Sterility, 51:317-323.

Eggert-Kruse W et al. (1992). Differentiation of round cells in semen by means of monoclonal antibodies and relationship with male fertility. Fertility and Sterility, 58:1046-1055.

Eggert-Kruse W et al. (1993). The $\mathrm{pH}$ as an important determinant of sperm-mucus interaction. Fertility and Sterility, 59:617-628.

Eggert-Kruse W et al. (1996). Sperm morphology assessment using strict criteria and male fertility under in-vivo conditions of conception. Human Reproduction, 11:139-146.

Eliasson R (1971). Standards for investigation of human semen. Andrologia, 3:49-64.

Eliasson R (1975). Analys is of semen. In: Behrman SJ, Kistner RW, eds. Progress in Infertility, 2nd ed. New York, Little, B rown: 691-713.

Eliasson R (1981). Analysis of semen. In: Burger H, de Kretser D, eds. The testis. New York, Raven Press: 381-399.

Eliasson R (2003). Basic semen analysis. In: Matson P, ed. Current topics in andrology. Perth, Ladybrook Publishing: 35-89.

Eliasson R et al. (1970). Empfehlungen zur Nomenklatur in der Andrologie. Andrologia, $2: 1257$.

ESHRE (1998). Guidelines on the application of CASA technology in the analysis of spermatozoa. Human Reproduction, 13:142-145.

ESHRE/NAFA (2002). Manual on basic semen analysis (ESHRE Monographs \#2). Oxford, Oxford University Press.

Evenson DP, Wixon R (2006). Clinical aspects of sperm DNA fragmentation detection and male infertility. Theriogenology, 65:979-991. 
Ezeh UI, Moore HM (2001). Redefining azoospermia and its implications. Fertility and Sterility, 75:213-214.

Ezeh UI et al. (1998). Correlation of testicular pathology and sperm extraction in azoospermic men with ejaculated spermatids detected by immunofluorescent localization. Human Reproduction, 13:3061-3065.

Feldschuh J et al. (2005). Successful sperm storage for 28 years. Fertility and Sterility, 84:1017.

Fenichel $P$ et al. (1989). Evaluation of the human sperm acrosome reaction using a monoclonal antibody, GB24, and fluorescence-activated cell sorter. J ournal of Reproduction and Fertility, 87:699-706.

Fetic $S$ et al. (2006). Relationship of cytoplasmic droplets to motility, migration in mucus, and volume regulation of human spermatozoa. J ournal of Andrology, 27:294-301.

Franken DR et al. (1989). The hemizona assay (HZA): a predictor of human sperm fertilizing potential in in-vitro fertilization (IVF) treatment. J ournal of In Vitro Fertilization and Embryo Transfer, 6:44-50.

Franken DR et al. (2000). Physiological induction of the acrosome reaction in human sperm: validation of a microassay using minimal volumes of solubilized, homologous zona pellucida. J ournal of Assisted Reproduction and Genetics, 17:374-378.

Fredricsson B, Björk G (1977). Morphology of postcoital spermatozoa in the cervical secretion and its clinical significance. Fertility and Sterility, 28:841-845.

Gandini L et al. (2000). Study of apoptotic DNA fragmentation in human spermatozoa. Human Reproduction, 15:830-839.

Gao DY et al. (1995). Prevention of osmotic injury to human spermatozoa during addition and removal of glycerol. Human Reproduction, 10:1109-1122.

Garrett C, Baker HWG (1995). A new fully automated system for the morphometric analysis of human sperm heads. Fertility and Sterility, 63:1306-1317.

Garrett C et al. (1997). Selectivity of the human sperm-zona pellucida binding process to sperm head morphometry. Fertility and Sterility, 67:362-371.

Garrett C et al. (2003). Auto mated semen analysis: "zona pellucida preferred" sperm morphometry and straight-line velocity are related to pregnancy rate in subfertile couples. Human Reproduction, 18:1643-1649.

Gilling-Smith C et al. (2005). Laboratory safety during assisted reproduction in patients with blood-borne viruses. Human Reproduction, 20:1433-1438.

Gilling-Smith C et al. (2006). HIV and reproductive care-a review of current practice. B ritish J ournal of Gynaecology, 113:869-878.

Gomez E et al. (1996). Development of an image analysis system to monitor the retention of residual cytoplasm by human spermatozoa: correlation with biochemical markers of the cytoplasmic space, oxidative stress, and sperm function. J ournal of Andrology, 17:276-287.

Gomez E et al. (1998). Evaluation of a spectrophotometric assay for the measurement of malondialdehyde and 4-hydroxyalkenals in human spermatozoa: relationships with semen quality and sperm function. International J ournal of Andrology, 21:81-94.

Gould J E et al. (1994). Sperm-immunobead binding decreases with in-vitro incubation. Fertility and Sterility, 62:167-171. 
Grimes DA, Lopez LM (2007). "Oligozoospermia," "azoospermia," and other semen-analysis terminology: the need for better science. Fertility and Sterility, 88:1491-1494.

Griveau J F, Le Lannou D (1997). Reactive oxygen species and human spermatozoa: physiology and pathology. International J ournal of Andrology, 20:61-69.

Handelsman DJ et al. (1984). Testicular function in potential sperm donors: normal ranges and the effects of smoking and varicocele. International J ournal of Andrology, 7:369-382.

Haugen TB, Grotmol T (1998). pH of human semen. International J ournal of Andrology, 21:105-108.

Heite H-J , Wetterauer W (1979). Acid phosphatase in seminal fluid: method of estimation and diagnostic significance. Andrologia, 11:113-122.

Hellstrom WJ G et al. (1989). A comparison of the usefulness of SpermMar and Immunobead tests for the detection of antisperm antibodies. Fertility and Sterility, 52:1027-1031.

Henkel R et al. (2004). Influence of deoxyribonucleic acid damage on fertilization and pregnancy. Fertility and Sterility, 81:965-972.

Henley $\mathrm{N}$ et al. (1994). Flow cytometric evaluation of the acrosome reaction of human spermatozoa: a new method using a photoactivated supravital stain. International J ournal of Andrology, 17:78-84.

Holstein AF et al. (2003). Understanding spermatogenesis is a prerequisite for treatment. Reproductive Biology and Endocrinology, 1:107.

Homyk M et al. (1990). Differential diagnosis of immature germ cells in semen utilizing monoclonal antibody MHS-10 to the intra-acrosomal antigen SP-10. Fertility and Sterility, 53:323-330.

Hossain AM et al. (1998). Time course of hypo-osmotic swellings of human spermatozoa: evidence of ordered transition between swelling subtypes. Human Reproduction, 13:1578-1583.

Hotchkiss RS (1945). Fertility in men. London, William Heineman Medical Books.

Huggins C et al. (1942). Chemical composition of human semen and of the secretions of the prostate and seminal vesicles. American J ournal of Physiology, 136:467-473.

IARC (1982). Chemicals, industrial processes and industries associated with cancer in humans. Lyon, International Agency for Research on Cancer (IARC Monographs on the Evaluation of Carcinogenic Risks to Humans, Suppl. 4): 169-170.

Insler V et al. (1972). The cervical score. A simple semiquantitative method for monitoring of the menstrual cycle. International J ournal of Gynaecology and Obstetrics, $10: 223-228$.

Irvine DS et al. (1994). A prospective clinical study of the relationship between the computer-assisted assessment of human semen quality and the achievement of pregnancy in vivo. Human Reproduction, 9:2324-2334.

Ivic A et al. (2002). Critical evaluation of methylcellulose as an alternative medium in sperm migration tests. Human Reproduction, 17:143-149.

Iwamoto T et al. (2006). Semen quality of 324 fertile J apanese men. Human Reproduction, 21:760-765.

Iwasaki A, Gagnon C (1992). Formation of reactive oxygen species in spermatozoa of infertile patients. Fertility and Sterility, 57:409-416. 
J affe TM et al. (1998). Sperm pellet analysis: a technique to detect the presence of sperm in men considered to have azoospermia by routine semen analysis. J ournal of Urology, 159:1548-1550.

J eyendran RS et al. (1984). Development of an assay to assess the functional integrity of the human sperm membrane and its relationship to the other semen characteristics. J ournal of Reproduction and Fertility, 70:219-228.

J ohanisson E et al. (2000). Evaluation of "round cells" in semen analysis: a comparative study. Human Reproduction Update, 6:404-412.

J ohnsen O, Eliasson R (1987). Evaluation of a commercially available kit for the colorimetric determination of zinc in human seminal plasma. International J ournal of Andrology, 10:435-440.

J ohnson DE et al. (1996). Glass wool column filtration versus mini-Percoll gradient for processing poor quality semen samples. Fertility and Sterility, 66:459-462.

J ones DM et al. (1986). Immobilization of sperm by condoms and their components. Clinical Reproduction and Fertility, 4:367-372.

J ones R et al. (1979). Peroxidative breakdown of phospholipids by human spermatozoa, spermicidal properties of fatty acid peroxides and protective action of seminal plasma. Fertility and Sterility, 31:531-537.

J ørgensen $\mathrm{N}$ et al. (2001). Regional differences in semen quality in Europe. Human Reproduction, 16:1012-1019.

J ouannet $P$ et al. (1988). Male factors and the likelihood of pregnancy in infertile couples. I. Study of sperm characteristics. International J ournal of Andrology, 11:379-394.

Kamischke A et al. (2004). Cryopreservation of sperm from adolescents and adults with malignancies. J ournal of Andrology, 25:586-592.

Karvonen MJ , Malm M (1955). Colorimetric determination of fructose with indol. Scandinavian J ournal of Clinical Laboratory Investigation, 7:305-307.

Katz DF et al. (1986). Morphometric analysis of spermatozoa in the assessment of human male fertility. J ournal of Andrology, 7:203-210.

Keel BA (2006). Within- and between-subject variation in semen parameters in infertile men and normal semen donors. Fertility and Sterility, 85:128-134.

Keel BA, Webster BW (1993). Semen cryopreservation methodology and results. In: Barratt CLR, Cooke ID, eds. Donor insemination. Cambridge, Cambridge University Press: 71-96.

Kraemer M et al. (1998). Factors influencing human sperm kinematic measurements by the Celltrak computer-assisted sperm analysis system. Human Reproduction, 13:611-619.

Krause W (1995). Computer-assisted sperm analysis system: comparison with routine evaluation and prognostic value in male fertility and assisted reproduction. Human Reproduction, 10 (Suppl. 1): 60-66.

Krausz C et al. (1992). Development of a technique for monitoring the contamination of human semen samples with leucocytes. Fertility and Sterility, 57:1317-1325.

Kremer J (1965). A simple sperm penetration test. International J ournal of Fertility, 10:209-215.

Kremer J , J ager S (1980). Characteristics of anti-spermatozoal antibodies responsible for the shaking phenomenon, with special regard to immunoglobulin class and antigenreactive sites. International J ournal of Andrology, 3:143-152. 
Kruger TF et al. (1986). Sperm morphologic features as a prognostic factor in in-vitro fertilization. Fertility and Sterility, 46:1118-1123.

Kruger TF et al. (1987). A quick, reliable staining technique for human sperm morphology. Archives of Andrology, 18:275-277.

Kruger TF et al. (1991). Hemizona assay: use of fresh versus salt-stored human oocytes to evaluate sperm binding potential to the zona pellucida. J ournal of In Vitro Fertilization and Embryo Transfer, 8:154-156.

Kruger TF et al. (1993). The self teaching programme for strict sperm morphology. B ellville, South Africa, MQ Medical.

Kuster CE et al. (2004). Determining sample size for the morphological assessment of sperm. Theriogenology, 61:691-703.

Lacquet FA et al. (1996). Slide preparation and staining procedures for reliable results using computerized morphology. Archives of Andrology, 36:133-138.

Larsen $L$ et al. (2000). Computer-assisted semen analysis parameters as predictors for fertility of men from the general population. The Danish First Pregnancy Planner Study Team. Human Reproduction, 15:1562-1567.

Le Lannou D, Lansac J (1993). Artificial procreation with frozen donor semen: the French experience of CECOS. In: Barratt CLR, Cooke ID, eds. Donor insemination. Cambridge, Cambridge University P ress: 152-169.

Le Lannou D et al. (1992). Effects of chamber depth on the motion pattern of human spermatozoa in semen or in capacitating medium. Human Reproduction, 7:1417-1421.

Lee J D et al. (1996). Analysis of chromosome constitution of human spermatozoa with normal and aberrant head morphologies after injection into mouse oocytes. Human Reproduction, 11:1942-1946.

Leibo SP et al. (2002). Cryopreservation of human spermatozoa. In: Vayena E et al., eds. Current practices and controversies in assisted reproduction. Geneva, World Health Organization: 152-165.

Lentner C (1981). Geigy scientific tables. Vol. 1:Units of measurement, body fluids, composition of the body, nutrition. Basle, Ciba-Geigy: 50.

Lindsay KS et al. (1995). Classification of azoospermic samples. Lancet, 345:1642.

Liu DY, Baker HWG (1988). The proportion of human sperm with poor morphology but normal intact acrosomes detected with Pisum sativum agglutinin correlates with fertilization in vitro. Fertility and Sterility, 50:288-293.

Liu DY, Baker HWG (1992a). Morphology of spermatozoa bound to the zona pellucida of human oocytes that failed to fertilize in vitro. J ournal of Reproduction and Fertility, 94:71-84.

Liu DY, Baker HW (1992b). Tests of human sperm function and fertilization in vitro. Fertility and Sterility, 58:465-483.

Liu DY, Baker HWG (1994). Disordered acrosome reaction of spermatozoa bound to the zona pellucida: a newly discovered sperm defect causing infertility with reduced sperm-zona pellucida penetration and reduced fertilization in vitro. Human Reproduction, 9:1694-1700.

Liu DY, Baker HWG (1996). Relationship between the zonae pellucida (ZP) and ionophore A23187-induced acrosome reaction with the ability of sperm to penetrate the ZP in men with normal sperm-ZP binding. Fertility and Sterility, 66:312-315. 
Liu DY, Baker HWG (2003). Disordered zona pellucida induced acrosome reaction and failure of in-vitro fertilization in patients with unexplained infertility. Fertility and Sterility, 79:74-80.

Liu DY, Baker HW (2004). High frequency of defective sperm-zona pellucida interaction in oligozoospermic infertile men. Human Reproduction, 19:228-233.

Liu DY et al. (1988). A human sperm-zona pellucida binding test using oocytes that failed to fertilize in vitro. Fertility and Sterility, 50:782-788.

Liu DY et al. (1989). A sperm-zona pellucida binding test and in vitro fertilization. Fertility and Sterility, 52:281-287.

Liu DY et al. (1990). Use of oocytes that failed to be fertilized in vitro to study human sperm-oocyte interactions: comparison of sperm-oolemma and sperm-zona pellucida binding, and relationship with results of IVF. Reproduction, Fertility, Development, 2:641-650.

Liu DY et al. (1991a). Relationship between sperm motility assessed with the Hamilton Thorn motility analyzer and fertilization rates in vitro. J ournal of Andrology, $12: 231-239$.

Liu DY et al. (1991b). Horse and marmoset sperm bind to the zona pellucida of salt stored human oocytes. Fertility and Sterility, 56:764-767.

Liu DY et al. (2003). Low proportions of sperm can bind to the zona pellucida of human oocytes. Human Reproduction, 18:2382-2389.

Liu DY et al. (2004). Clinical application of sperm-oocyte interaction tests in in-vitro fertilization-embryo transfer and intracytoplasmic sperm injection programs. Fertility and Sterility, 82:1251-1263.

MacLeod J , Wang Y (1979). Male fertility potential in terms of semen quality: a review of the past, a study of the present. Fertility and Sterility, 31:103-116.

MacMillan RA, Baker HW (1987). Comparison of latex and polyacrylamide beads for detecting sperm antibodies. Clinical Reproduction and Fertility, 5:203-209.

Mahadevan M et al. (1981). Noninvasive method of semen collection for successful artificial insemination in a case of retrograde ejaculation. Fertility and Sterility, 36:243-247.

Mahmoud AM et al. (1997). The performance of 10 different methods for the estimation of sperm concentration. Fertility and Sterility, 68:340-345.

Martin RH et al. (2003). A comparison of the frequency of sperm chromosome abnormalities in men with mild, moderate, and severe oligozoospermia. Biology of Reproduction, 69:535-539.

Matson PL (1995). External quality assessment for semen analysis and sperm antibody detection: results of a pilot scheme. Human Reproduction, 10:620-625.

McKinney KA et al. (1996). Reactive oxygen species generation in human sperm: luminol and lucigenin chemiluminescence probe. Archives of Andrology, 36:119-125.

Meinertz H, Bronson R (1988). Detection of antisperm antibodies on the surface of motile spermatozoa. Comparison of the immunobead binding technique (IBT) and the mixed antiglobulin reaction (MAR). A meric an J ournal of Reproductive Immunology and Microbiology, 18:120-123.

Menkveld R, Kruger TF (1990). Basic semen analysis. In: Acosta AA et al., eds. Human spermatozoa in assisted reproduction. Baltimore, Williams \& Wilkins: 68-84. 
Menkveld R, Kruger TF (1996). Evaluation of sperm morphology by light microscopy. In: Acosta AA, Kruger TF, eds. Human spermatozoa in assisted reproduction, 2nd ed. London, Parthenon Publishing: 89-107.

Menkveld R et al. (1990). The evaluation of morphological characteristics of human spermatozoa according to stricter criteria. Human Reproduction, 5:586-592.

Menkveld R et al. (1991). Sperm selection capacity of the human zona pellucida. Molecular Reproduction and Development, 30:346-352.

Menkveld R et al. (1997). Effects of different staining and washing procedures on the results of human sperm morphology by manual and computerized methods. Andrologia, 29:1-7.

Menkveld R et al. (2001). Semen parameters, including WHO and strict criteria morphology, in a fertile and subfertile population: an effort towards standardization of in-vivo thresholds. Human Reproduction, 16:1165-1171.

Meschede D et al. (1993). Influence of three different preparation techniques on the results of human sperm morphology analysis. International J ournal of Andrology, 16:362-369.

Meseguer M et al. (2006). S perm cryopreservation in oncological patients: a 14-year followup study. Fertility and Sterility, 85:640-645.

Moghissi KS (1976). Post-coital test: physiological basis, technique and interpretation. Fertility and Sterility, 27:117-129.

Moghissi KS et al. (1964). Mechanism of sperm migration. Fertility and Sterility, 15:15-23.

Möllering H, Gruber W (1966). Determination of citrate with citrate lyase. Analytical Biochemistry, 17:369-376.

Morshedi M et al. (2003). Efficacy and pregnancy outcome of two methods of semen preparation for intrauterine insemination: a prospective randomized study. Fertility and Sterility, 79 (Suppl. 3): 1625-1632.

Mortimer D (1994a). Practical laboratory andrology. Oxford, Oxford University Press.

Mortimer D (1994b). Laboratory standards in routine clinical andrology. Reproductive Medicine Review, 3:97-111.

Mortimer D (2004). Current and future concepts and practices in human sperm cryobanking. Reproductive Biomedicine Online, 9:134-151.

Mortimer D, Menkveld R (2001). Sperm morphology assessment-historical perspectives and current opinions. J ournal of Andrology, 22:192-205.

Mortimer D, Mortimer S (2005). Quality and risk management in the IVF laboratory. Cambridge, Cambridge University Press.

Mortimer D et al. (1995). Workshop report: clinical CASA-the quest for consensus. Reproduction, Fertility and Development, 7:951-959.

Nahoum CRD, Cardozo D (1980). Staining for volumetric count of leukocytes in semen and prostate-vesicular fluid. Fertility and Sterility, 34:68-69.

Neuwinger J et al. (1990). External quality control in the andrology laboratory: an experimental multicenter trial. Fertility and Sterility, 54:308-314.

Neuwinger J et al. (1991). Hyaluronic acid as a medium for human sperm migration tests. Human Reproduction, 6:396-400. 
$\mathrm{Ng}$ FL et al. (1992). Comparison of Percoll, mini-Percoll and swim-up methods for sperm preparation from abnormal semen samples. Human Reproduction, 7:261-266.

$\mathrm{Ng}$ KK et al. (2004). Sperm output of older men. Human Reproduction, 19:1811-1815.

Oehninger S et al. (1993). The specificity of human spermatozoa/zona pellucida interaction under hemi-zona assay conditions. Molecular Reproduction and Development, 35:57-61.

Oei SG et al. (1995). When is the post-coital test normal? A critical appraisal. Human Reproduction, 10:1711-1714.

Overstreet J W et al. (1980). In-vitro capacitation of human spermatozoa after passage through a column of cervical mucus. Fertility and Sterility, 34:604-606.

Paquin R et al. (1984). Similar biochemical properties of human seminal plasma and epididymal $\alpha 1,4-$ glucosidase. J ournal of Andrology, 5:277-282.

Pelfrey RJ et al., (1982). Abnormalities of sperm morphology in cases of persistent infertility after vasectomy reversal. Fertility and Sterility, 38:112-114.

Pérez-Sánchez F et al. (1994). Improvement in quality of cryopreserved human spermatozoa by swim-up before freezing. International J ournal of Andrology, 17:115-120.

Perloff WH, Steinberger E (1963). In-vitro penetration of cervical mucus by spermatozoa. Fertility and Sterility, 14:231-236.

Perloff WH et al. (1964). Conception with human spermatozoa frozen by nitrogen vapor technique. Fertility and Sterility, 15:501-504.

Plaut DA, Westgard J OW (2002). QC external quality assessment. In: Westgard J O, ed. B asic QC practices: training in statistical quality control for healthcare laboratories. Madison, WI, QC Publishing: 125-163.

Poland ML et al. (1985). Variation of semen measures within normal men. Fertility and Sterility, 44:396-400.

Polge $C$ et al. (1949). Revival of spermatozoa after vitrification and dehydration at low temperatures. Nature, 164:626-627.

Pound $\mathrm{N}$ et al. (2002). Duration of sexual arousal predicts semen parameters for masturbatory ejaculates. Physiology and Behavior, 76:685-689.

Punab M et al. (2003). The limit of leucocytospermia from the microbiological viewpoint. Andrologia, 35:271-278.

Quinn P et al. (1985). Improved pregnancy rate in human in-vitro fertilization with the use of a medium based on the composition of human tubal fluid. Fertility and Sterility, 44:493-498.

Rajah SV et al. (1992). Comparison of mixed antiglobulin reaction and direct immunobead test for detection of sperm-bound antibodies in subfertile males. Fertility and Sterility, 57:1300-1303.

Rao B et al. (1989). Lipid peroxidation in human spermatozoa as related to midpiece abnormalities and motility. Gamete Research, 24:127-134.

Rhemrev J et al. (1989). Human sperm selection by glass wool filtration and two-layer discontinuous Percoll gradient centrifugation. Fertility and Sterility, 51:685-690.

Rose NR et al. (1976). Techniques for detection of iso- and auto-antibodies to human spermatozoa. Clinical and Experimental Immunology, 23:175-199. 
Rossi AG, Aitken RJ (1997). Interactions between leukocytes and the male reproductive system. The unanswered questions. Advances in Experimental Medicine and Biology, 424:245-252.

Said TM et al. (2004). Human sperm superoxide anion generation and correlation with semen quality in patients with male infertility. Fertility and Sterility, 82:871-877.

Sakkas D et al. (1998). Sperm nuclear DNA damage and altered chromatin structure: effect on fertilization and embryo development. Human Reproduction, 13(Suppl. 4):11-19.

Savasi V et al. (2007). Safety of sperm washing and ART outc ome in 741 HIV-1serodiscordant couples. Human Reproduction, 22:772-777.

Sawyer DE et al. (2003). Quantitative analysis of gene-specific DNA damage in human spermatozoa. Mutation Research, 529:21-34.

Scarselli G et al. (1987). Approach to immunological male infertility: a comparison between MAR test and direct immunobead test. Acta Europea Fertilitatis, 18:55-57.

Schmidt KL et al. (2004). Assisted reproduction in male cancer survivors: fertility treatment and outcome in 67 couples. Human Reproduction, 19:2806-2810.

Seaman EK et al. (1996). Accuracy of semen counting chambers as determined by the use of latex beads. Fertility and Sterility, 66:662-665.

Shah VP et al. (2000). Bioanalytical method validation-a revisit with a decade of progress. Pharmacological Research, 17:1551-1557.

Sharif K (2000). Reclassification of azoospermia: the time has come? Human Reproduction, $15: 237-238$

Sharma RK et al. (2001). Relationship between seminal white blood cell counts and oxidative stress in men treated at an infertility clinic. J ournal of Andrology, 22:575-583.

Sherman J K (1990). Cryopreservation of human semen. In: Keel BA, Webster BW, eds. CRC handbook of the laboratory diagnosis and treatment of infertility. Boca Raton, CRC Press: 229-259.

Shibahara $\mathrm{H}$ et al. (2004). Prediction of pregnancy by intrauterine insemination using CASA estimates and strict criteria in patients with male factor infertility. International J ournal of Andrology, 27:63-68.

Slama R et al. (2002). Time to pregnancy and semen parameters: a cross-sectional study among fertile couples from four European cities. Human Reproduction, 17:503-515.

S mith R et al. (1996). Total antioxidant capacity of human seminal plasma. Human Reproduction, 11:1655-1660.

Sobrero AJ , MacLeod J (1962). The immediate postcoital test. Fertility and Sterility, 13:184-189.

Soler $C$ et al. (2000). Objective evaluation of the morphology of human epididymal sperm heads. International J ournal of Andrology, 23:77-84.

Tedder RS (1995). Hepatitis B transmission from contaminated cryopreservation tank. Lancet, 346:137-140.

Tomlinson M (2005). Managing risk associated with cryopreservation. Human Reproduction, 20:1751-1756. 
Tomlinson MJ et al. (1993). Prospective study of leukocytes and leukocyte subpopulations in semen suggests they are not a cause of male infertility. Fertility and Sterility, 60:1069-1075.

Toner J P et al. (1995). Value of sperm morphology assessed by strict criteria for prediction of the outcome of artificial (intrauterine) insemination. Andrologia, 27:143-148.

Tyler J P et al. (1982a). Studies of human seminal parameters with frequent ejaculation. I. Clinical characteristics. Clinical Reproduction and Fertility, 1:273-285.

Tyler J P et al. (1982b). Studies of human seminal parameters with frequent ejaculation II. Spermatozoal vitality and storage. Clinical Reproduction and Fertility, 1:287-293.

Van der Merwe FH et al. (2005). The use of semen parameters to identify the subfertile male in the general population. Gynecologic and Obstetric Investigation, 59:86-91.

Van Waart J et al. (2001). Predictive value of normal sperm morphology in intrauterine insemination (IUI): a structured literature review. Human Reproduction Update, 7:495-500.

Verheyen $\mathrm{G}$ et al. (1993). Effect of freezing method, thawing temperature and post-thaw dilution/washing on motility (CASA) and morphology characteristics of high-quality human sperm. Human Reproduction, 8:1678-1684.

Virro MR et al. (2004). Sperm chromatin structure assay (SCSA) parameters are related to fertilization, blastocyst development, and ongoing pregnancy in in-vitro fertilization and intracytoplasmic sperm injection cycles. Fertility and Sterility, 81:1289-1295.

von der Kammer H et al. (1991). The evaluation of markers of prostatic function. Urological Research, 19:343-347.

von Eckardstein S et al. (2000). Seminal plasma characteristics as indicators of cystic fibrosis transmembrane conductance regulator (CFTR) gene mutations in men with obstructive azoospermia. Fertility and Sterility, 73:1226-1231.

Watson PF (1995). Recent developments and concepts in the cryopreservation of spermatozoa and the assessment of their post-thawing function. Reproduction Fertility and Development, 7:871-891.

Weiske WH (1994). Minimal invasive Vasektomie mittels Fulgurationstechnik. Erfahrungen bei 1000 Patienten in $12 \mathrm{~J}$ ahren. Urologe, B 34:448-452.

Weiske WH et al. (2000). Clinical findings in congenital absence of the vasa deferentia. Andrologia, 32:13-18.

Westgard J O (2002). Foreword to the second edition. In: Westgard J O, ed. Basic QC practic es: training in statistical quality control for healthcare laboratories. Madison, WI, QC Publishing.

Wheeler DJ (1993). Understanding variation: the key to managing chaos. Knoxville, TN, SPC Press.

Wheeler DJ , Chambers DS (1992). Understanding statistical process control, 2nd ed. Knoxville, TN, SPC Press.

WHO (1986). Consultation on the zona-free hamster oocyte penetration test and the diagnosis of male fertility. International J ournal of Andrology, (Suppl. 6).

WHO (1987). (prepared by Comhaire F et al.) Towards more objectivity in diagnos is and management of male infertility. International J ournal of Andrology, (Suppl. 7): 22-24. 
WHO (1996). Task Force for the Regulation of Male Fertility. Contraceptive efficacy of testosterone-induced azoospermia and oligozoospermia in normal men. Fertility and Sterility, 65:821-829.

WHO (1999). Laboratory manual for the examination of human semen and sperm-cervical mucus interaction, 4th ed. Cambridge, Cambridge University Press.

WHO (2004). Laboratory biosafety manual, 3rd ed. Geneva, World Health Organization (http://whqlibdoc.who.int/publications/2004/9241546506.pdf, last accessed 25 February 2010).

Wilton LJ et al. (1988). Human male infertility caused by degeneration and death of sperms in the epididymis. Fertility and Sterility, 49:1051-1058.

Wolf DP (1995). Semen cryopreservation. In: Keye WR et al., eds. Infertility evaluation and treatment. Philadelphia, WB Saunders: 686-695.

Wolff $\mathrm{H}$ (1995). The biologic signific ance of white blood cells in semen. Fertility and Sterility, 63:1143-1157.

Woods EJ et al. (2004). Fundamental cryobiology of reproductive cells and tissues. Cryobiology, 48:146-156.

Yanagimachi R et al. (1979). Retention of biologic characteristics of zona pellucida in highly concentrated salt solution: the use of salt stored eggs for assessing the fertilizing capacity of spermatozoa. Fertility and Sterility, 31:562-574.

Zavos PM, Goodpasture J C (1989). Clinical improvements of specific seminal deficiencies via intercourse with a seminal collection device versus masturbation. Fertility and Sterility, 51:190-193.

Zinaman MJ et al. (1996). Evaluation of computer-assisted semen analysis (CASA) with IDENT stain to determine sperm concentration. J ournal of Andrology, 17:288-292.

Zinaman MJ et al. (2000). Semen quality and human fertility: a prospective study with healthy couples. J ournal of Andrology, 21:145-153.

Zorn B et al. (2003). Seminal reactive oxygen species as predictors of fertilization, embryo quality and pregnancy rates after conventional in-vitro fertilization and intracytoplasmic sperm injection. International J ournal of Andrology, 26:279-285. 
Appendices 



\section{APPENDIX $1 \quad$ Reference values and semen nomenclature}

\section{A1.1 Reference values}

Measurements made on semen samples need to be compared with reference values to allow decisions to be made about patient management and thresholds for clinical trials or investigations. The reference values given here have been generated from the results of several prospective, cross-sectional studies of semen quality and fertility. They were obtained by direct, retrospective selection of fertile men, defined as men whose partner conceived within 12 months after stopping use of contraception (Cooper et al., 2010).

- Only complete semen samples-one per man (the first where several were given), obtained following 2-7 days of abstinence-were included in this analysis.

- Semen volume was measured using methods recommended by WHO at the time, namely, weighing or transferring to pipettes or graduated vessels. Total sperm number was calculated from concentrations measured by haemocytometer on fixed, diluted samples. Total motility ( $R R+N P)$, progressive motility $(P R)$, non-progressive motility (NP) and immotile sperm (IM) were measured at room temperature or at $37^{\circ} \mathrm{C}$. Data on normal sperm morphology were taken only from laboratories that provided values not exceeding the anticipated maximum level for the strict categorization (Tygerberg) method (approximately 35\% normal forms). Vitality was determined by exclusion of vital dye (eosin) from sperm head membranes.

- Statistical tradition is to take the 2.5th centile from a two-sided reference interval as the threshold below which values may be considered to come from a different population. However, a one-sided reference interval was considered to be more appropriate for semen parameters, since high values are unlikely to be detrimental to fertility. The 5 th centile lower reference limits are given in Table A1.1, and the complete frequency distributions are given in Table A1.2.

Comment 1: The reference distributions in Table A1.2 provide a description of the semen characteristics of recent fathers, whose partner became pregnant within 12 months of stopping use of contraception.

Comment 2: Fathers constitute a select group of individuals and their semen parameters may be different from those of the general population of healthy men.

Comment 3: Semen characteristics are highly variable, both within and among men, and are not the sole determinants of a couple's fertility; the ranges therefore provide only a guide to a man's fertility status.

Comment 4: Semen parameters that lie within the $95 \%$ reference interval do not guarantee fertility. 
Comment 5: Men whose semen characteristics fall below the lower limits given here are not necessarily infertile; their semen characteristics are below the reference range for recent fathers - as are, by definition, those of $5 \%$ of the fertile men who provided data used in the calculation of the reference range.

Comment 6: A man's semen characteristics need to be interpreted in conjunction with clinical information.

Comment 7: There may be regional differences in semen quality, or differences between laboratories; laboratories should consider preparing their own reference ranges, using the techniques described in this manual.

Comment 8: Time to pregnancy is also affected by the female partner's fertility status.

Table A1.1 Lower reference limits (5th centiles and their 95\% confidence intervals) for semen characteristics

\begin{tabular}{|l|l|}
\hline Parameter & Lower reference limit \\
\hline Semen volume $(\mathrm{ml})$ & $1.5(1.4-1.7)$ \\
\hline Total sperm number $\left(10^{6}\right.$ per ejaculate) & $39(33-46)$ \\
\hline Sperm concentration $\left(10^{6}\right.$ per $\left.\mathrm{ml}\right)$ & $15(12-16)$ \\
\hline Total motility (PR +NP, \%) & $40(38-42)$ \\
\hline Progressive motility (PR, \%) & $32(31-34)$ \\
\hline Vitality (live spermatozoa, \%) & $58(55-63)$ \\
\hline Sperm morphology (normal forms, \%) & $4(3.0-4.0)$ \\
\hline Other consensus threshold values & \\
\hline pH & $\geq 7.2$ \\
\hline Peroxidase-positive leukocytes (106 per ml) & $<1.0$ \\
\hline MAR test (motile spermatozoa with bound particles, \%) & $<50$ \\
\hline Immunobead test (motile spermatozoa with bound beads, \%) & $<50$ \\
\hline Seminal zinc ( $\mu$ mol/ejaculate) & $\geq 2.4$ \\
\hline Seminal fructose ( $\mu$ mol/ejaculate) & $\geq 13$ \\
\hline Seminal neutral glucosidase (mU/ejaculate) & $\geq 20$ \\
\hline
\end{tabular}


Table A1.2 Distribution of values for semen parameters from men whose partners became pregnant within 12 months of discontinuing contraceptive use

\begin{tabular}{|c|c|c|c|c|c|c|c|c|c|c|}
\hline \multirow{2}{*}{ Parameter (units) } & \multirow{2}{*}{$\mathbf{N}$} & \multicolumn{9}{|c|}{ Centile } \\
\hline & & 2.5 & 5 & 10 & 25 & 50 & 75 & 90 & 95 & 97.5 \\
\hline Semen volume (ml) & 1941 & 1.2 & 1.5 & 2.0 & 2.7 & 3.7 & 4.8 & 6.0 & 6.8 & 7.6 \\
\hline $\begin{array}{l}\text { Total sperm number } \\
\text { (106 per ejaculate) }\end{array}$ & 1859 & 23 & 39 & 69 & 142 & 255 & 422 & 647 & 802 & 928 \\
\hline $\begin{array}{l}\text { Sperm concentration } \\
\left(10^{6} \text { per } \mathrm{ml}\right)\end{array}$ & 1859 & 9 & 15 & 22 & 41 & 73 & 116 & 169 & 213 & 259 \\
\hline Total motility (PR +NP, \% ) & 1781 & 34 & 40 & 45 & 53 & 61 & 69 & 75 & 78 & 81 \\
\hline Progressive motility $(P R, \%)$ & 1780 & 28 & 32 & 39 & 47 & 55 & 62 & 69 & 72 & 75 \\
\hline $\begin{array}{l}\text { Non-progressive motility } \\
(\mathrm{NP}, \%)\end{array}$ & 1778 & 1 & 1 & 2 & 3 & 5 & 9 & 15 & 18 & 22 \\
\hline Immotile spermatozoa (IM, \%) & 1863 & 19 & 22 & 25 & 31 & 39 & 46 & 54 & 59 & 65 \\
\hline Vitality (\%) & 428 & 53 & 58 & 64 & 72 & 79 & 84 & 88 & 91 & 92 \\
\hline Normal forms (\%) & 1851 & 3 & 4 & 5.5 & 9 & 15 & 24.5 & 36 & 44 & 48 \\
\hline
\end{tabular}

Source: Cooper et al., 2010.

\section{A1.2 Nomenclature}

This manual retains the nomenclature introduced to describe deviations from reference semen values, using words rather than numbers (see Table A1.3), although some have argued for the abandonment of such terminology (Grimes \& Lopez, 2007). The nomenclature simply classifies the quality of the semen and does not suggest any biological cause (Eliasson et al., 1970). These terms are used to describe samples with values lying outside the reference range, and therefore possibly originating from a different population. Much of the semen nomenclature relates to a single parameter. However, normozoospermia refers to three sperm parameters-number, motility and morphology. Thus deviations from the reference range for each parameter can be described individually.

\section{References}

Cooper TG et al. (2010). World Health Organization reference values for human semen characteristics. Human Reproduction Update, 16:231-245.

Grimes DA, Lopez LM (2007). “Oligozoospermia”, “azoospermia”, and other semen-analysis terminology: the need for better science. Fertility and Sterility, 88:1491-1494.

Eliasson R et al. (1970). Empfehlungen zur Nomenklatur in der Andrologie. Andrologia, 2:1257. 
Table A1.3 Nomenclature related to semen quality

\begin{tabular}{|c|c|}
\hline aspermia & no semen (no or retrograde ejaculation) \\
\hline asthenozoospermia & $\begin{array}{l}\text { percentage of progressively motile (PR) spermatozoa below the lower } \\
\text { reference limit }\end{array}$ \\
\hline asthenoteratozoospermia & $\begin{array}{l}\text { percentages of both progressively motile }(P R) \text { and morphologically } \\
\text { normal spermatozoa below the lower reference limits }\end{array}$ \\
\hline azoospermia & $\begin{array}{l}\text { no spermatozoa in the ejaculate (given as the limit of quantification for } \\
\text { the assessment method employed) }\end{array}$ \\
\hline cryptozoospermia & $\begin{array}{l}\text { spermatozoa absent from fresh preparations but observed in a centri- } \\
\text { fuged pellet }\end{array}$ \\
\hline $\begin{array}{l}\text { haemospermia } \\
\text { (haematospermia) }\end{array}$ & presence of erythrocytes in the ejaculate \\
\hline $\begin{array}{l}\text { leukospermia (leukocyto- } \\
\text { spermia, pyospermia) }\end{array}$ & presence of leukocytes in the ejaculate above the threshold value \\
\hline necrozoospermia & $\begin{array}{l}\text { low percentage of live, and high percentage of immotile, spermatozoa } \\
\text { in the ejaculate }\end{array}$ \\
\hline normozoospermia & $\begin{array}{l}\text { total number (or concentration, depending on outcome reported)* of } \\
\text { spermatozoa, and percentages of progressively motile }(P R) \text { and mor- } \\
\text { phologically normal spermatozoa, equal to or above the lower reference } \\
\text { limits }\end{array}$ \\
\hline oligoasthenozoospermia & $\begin{array}{l}\text { total number (or concentration, depending on outcome reported)* of } \\
\text { spermatozoa, and percentage of progressively motile (PR) spermato- } \\
\text { zoa, below the lower reference limits }\end{array}$ \\
\hline $\begin{array}{l}\text { oligoasthenoterato- } \\
\text { zoospermia }\end{array}$ & $\begin{array}{l}\text { total number (or concentration, depending on outcome reported)* of } \\
\text { spermatozoa, and percentages of both progressively motile (PR) and } \\
\text { morphologically normal spermatozoa, below the lower reference limits }\end{array}$ \\
\hline oligoteratozoospermia & $\begin{array}{l}\text { total number (or concentration, depending on outcome reported)* of } \\
\text { spermatozoa, and percentage of morphologically normal spermatozoa, } \\
\text { below the lower reference limits }\end{array}$ \\
\hline oligozoospermia & $\begin{array}{l}\text { total number (or concentration, depending on outcome reported)* of } \\
\text { spermatozoa below the lower reference limit }\end{array}$ \\
\hline teratozoospermia & $\begin{array}{l}\text { percentage of morphologically normal spermatozoa below the lower } \\
\text { reference limit }\end{array}$ \\
\hline
\end{tabular}

*P reference should always be given to total number, as this parameter takes precedence over concentration.

Note: The suffix "spermia" refers to the ejaculate and "zoospermia" to the spermatozoa. Thus, the following terms should not be used: asthenospermia, asthenoteratospermia, cryptospermia, oligoasthenospermia, oligoteratospermia, oligospermia, teratospermia. 


\section{APPENDIX 2 Equipment and safety}

\section{A2.1 Basic supplies needed in an andrology laboratory}

Below is a list of the supplies and equipment needed in an andrology laboratory to perform the basic tests described in this manual.

Consult the published scientific literature referenced in this manual or elsewhere if you require assistance in finding a source of any of the following supplies.

A2.1.1 The laboratory should have the following general equipment and supplies:

- balance;

- benches with impermeable work surfaces;

- containers:

- for disposal of sharp objects;

- for hazardous waste;

- copy of Laboratory biosafety manual (WHO, 2004);

- deep freezer;

- disinfectant or sodium hypochlorite, $0.1 \%(\mathrm{v} / \mathrm{v})$ and $1 \%(\mathrm{v} / \mathrm{v})$ in purified water;

- disinfectant soap or antiseptic skin cleanser;

- disposable gloves;

- eye-wash solution or eye-rinse;

- first-aid kit;

- fume cupboard for storage of, and for working with, toxic reagents, chemicals or dyes;

- refrigerator;

- shower.

A2.1.2 The following supplies and equipment are needed for semen analysis:

- capillary tubes and sealant (for mucus penetration assay);

- CASA machine (optional);

- centrifuges:

- bench centrifuge capable of achieving 300-500g (for routine sperm handling and for urine), $1000 \mathrm{~g}$ (for semen markers) and $2000 \mathrm{~g}$ (for viscous samples);

- higher-speed centrifuge reaching $3000 \mathrm{~g}$ (for preparing suspected azoospermic samples) or microcentrifuge reaching $16000 \mathrm{~g}$ (for obtaining sperm-free seminal plasma) (see Box A2.1);

- cryoconservation equipment (optional); 
- condoms: spermicide-free, non-toxic (optional);

- dilution vials;

- dissecting microscope (optional; for collecting hamster oocytes);

- filter paper, $90 \mathrm{~g} / \mathrm{m}^{2}$ (for filtering stains);

- fluorescence microscope and objectives (optional; for high-sensitivity sperm concentration measurements and acrosome reaction tests);

- haemocytometers: improved Neubauer or alternative, $100 \mu \mathrm{m}$ deep, with thick coverslip (thickness number 4, $0.44 \mathrm{~mm}$ );

- incubator $\left(37^{\circ} \mathrm{C}\right)$, preferably with $5 \%$ (v/v) $\mathrm{CO}_{2}$ (optional);

- laboratory film: self-sealing, mouldable;

- laboratory multi-key counter(six or nine keys);

- large-volume counting chamber (optional; for assessing low sperm concentrations);

- luminometer (optional; for ROS assay);

- microscope slides:

- with ground glass or textured writing surface and coverslips (thickness number 1.5, 0.16-0.19 $\mathrm{mm}$ );

- plain slides for pulling a drop of semen over another slide to make semen smears;

- pen/pencils:

- for writing on frosted glass slides; a pencil with lead of softness HB (American rating number 2 ) is adequate;

- a wax/grease pencil (delimiting pen-optional; for limiting the area of antibody solution on a slide);

- permanent marker pen;

- $p H$ (ISFET) electrode (optional; for viscous semen samples);

- pH paper (range 6-10);

- phase-contrast microscope (for estimation of sperm concentration, motility, morphology) with at least a 50-watt light source and the following accessories (see Appendix 3):

- $\times 10, \times 20$ (or $\times 25$ ), $\times 40$ (or $\times 63$ ) positive-phase objectives, $\times 100$ oil-immersion objective;

- $\quad$ x40 negative- phase objective (optional; for eosin vitality test);

- wide-field X10 (or X12.5) eyepiece (ocular);

- eyepiece reticle (for judging area of field scanned for motility);

- stage micrometer (for sperm morphology measurement); 
- England finder (glass slide with grid-optional; for QC assessment);

- heated stage (optional; for measurement of sperm velocity);

- pipettes and pipette tips:

- Pasteur pipettes with latex droppers, or plastic disposable transfer pipettes, or automatic pipettes for mixing semen;

- air-displacement pipettes;

- positive-displacement pipettes to measure 10-100 $\mu$;

- record forms for results of semen and mucus analysis (see Appendix 6);

- sample mixers:

- two-dimensional shaker or rotating wheel for mixing semen (optional);

- vortex mixer for diluted semen;

- sealing tape for 96-well plates (optional; for fructose assay);

- semen collection container:

- disposable wide-mouth containers with lids;

- autoclavable glass collection cylinders;

- slide chambers, disposable (optional; for QC motility sample preparation);

- spectrophotometer (optional; for semen biochemistry assays);

- spot plate, porcelain or borosilicate glass (for eosin-nigrosin test);

- time generator (optional; for QC sample preparation);

- tissue paper: lint-free;

- warming plate: bench-top (optional; for prewarming slides for motility assessment).

A2.1.3 The following chemicals may be required:

- antibodies (CD45 for leukocytes);

- antifoaming agent (optional; for QC sample preparation);

- cellular peroxidase kit (optional);

- cryoprotective media (optional);

- density-gradient media (for sperm preparation);

- fructose assay kit (optional);

- glutaraldehyde (optional; for the HOP test);

- mineral oil (optional; for the HOP test);

- neutral $\alpha$-glucosidase assay kit (optional);

- Papanicolaou stain; 
- petroleum jelly (optional; for HOP test);

- rapid staining kit (optional; for sperm morphology);

- wax (melting point $48-66^{\circ} \mathrm{C}$ ) (optional; for HOP test);

- zinc assay kit (optional).

\section{Box A2.1 Calculating centrifugal forces}

The force to which spermatozoa are subjected during centrifugation (relative centrifugal force, RCF) depends on the speed of rotation ( $N$, revolutions per minute, r.p.m.) and the distance from the centre of the rotor to the point at which the force is to be measured (usually the bottom of the centrifuge tube) (radius, R, cm). RCF is calculated from the formula: $1.118 \times 10^{-5} \times \mathrm{R} \times \mathrm{N}^{2}$. For example, with a rotor radius of $8.6 \mathrm{~cm}$, centrifugation at 5000 r.p.m. will produce a force of $2404 \mathrm{~g}$; with a rotor radius of $13.5 \mathrm{~cm}$, centrifugation at 3900 r.p.m. will produce $2296 \mathrm{~g}$. Fig. A2.1 is a nomogram for determining RCF from the rotor radius and the speed of rotation.

\section{A2.2 Potential biohazards in an andrology laboratory}

Human body fluids, such as semen, are potentially infectious and should be handled and disposed of with special care. For the andrology laboratory, the most important infectious microorganisms that may be found in semen are HIV and hepatitis B and C viruses (HBV and HCV). Laboratory personnel should treat all biological samples as potentially infectious and should use appropriate caution in handling them.

\section{A2.3 Safety procedures for laboratory personnel}

- All laboratory personnel who work with human samples should be immunized against hepatitis $B$.

- No-one should eat, drink, smoke, apply cosmetics or store food in the andrology laboratory.

- Pipetting by mouth should not be permitted. Mechanical pipetting devices should always be used for the manipulation of liquids.

- All laboratory staff should wear a laboratory coat or disposable gown in the laboratory and remove it upon leaving. Laboratory personnel should wear disposable gloves (rubber, latex or vinyl, with or without powder), especially when handling fresh or frozen semen or seminal plasma or other biological samples and any containers that have come into contact with them. Gloves must be removed and discarded when staff leave the laboratory or use the telephone or computer. They must not be reused.

- Personnel should wash their hands regularly, especially before leaving the laboratory, after handling specimens and after removing gowns and gloves.

- Staff should take precautions to prevent accidental wounds from sharp instruments that may be contaminated with semen, and avoid contact of semen with open skin, cuts, abrasions or lesions. 
Fig. A2.1 Nomogram for determining relative centrifugal force (RCF) from rotor radius and rotation speed A straight line joining the rotor radius (cm, left axis) and rotation speed (r.p.m., right axis) intersects the middle axis at the RCF. In the example, a radius of $8 \mathrm{~cm}$ with a rotation speed of 2500 r.p.m. gives an RCF of approximately $550 \mathrm{~g}$. (The calculated value is $559 \mathrm{~g}$ (see Box A2.1)).
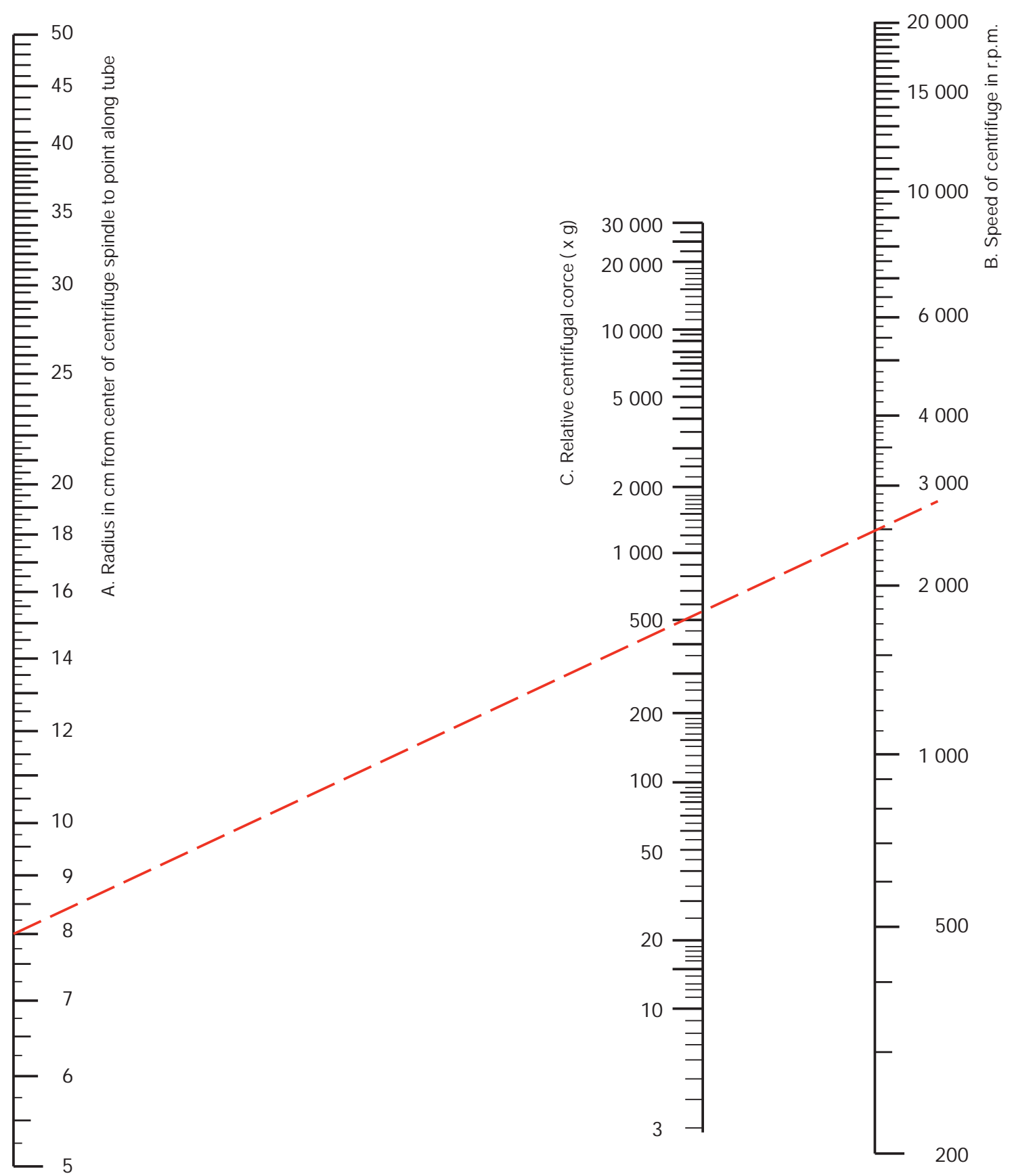
- Measures should be taken to prevent, and where necessary contain, spillages of semen or blood or urine samples.

- All sharp objects (needles, blades, etc.) should be placed in a marked container after use. This container should be sealed before it becomes full and disposed of in the same way as other dangerous laboratory items.

- All potentially hazardous items (gloves, semen containers) should be collected and disposed of appropriately.

- Face masks or surgical masks should be worn by all staff performing procedures that could potentially create aerosols or droplets, e.g. vortexing and centrifuging of open containers. The last drops of semen specimens should not be forcibly expelled from pipettes, because this can cause droplets or aerosols to form.

- Staff should wear protective safety goggles, insulated gloves and closed shoes when necessary, e.g. when using liquid nitrogen (see section A2.5).

\section{A2.4 Safety procedures for laboratory equipment}

Work surfaces and non-disposable vessels that have come into contact with semen or other biological samples should be sterilized or disinfected. The following procedures must be performed:

Daily, on completing the analyses:

- Wash the work space with disinfectant, e.g. sodium hypochlorite $0.1 \%$ ( $1 \mathrm{~g} / \mathrm{l})$ or similar disinfectant, wait at least 1 hour (or overnight), then rinse off disinfectant with water.

- Soak the counting chambers and coverslips in sodium hypochlorite $0.1 \%(1 \mathrm{~g} / \mathrm{l})$ or similar disinfectant overnight. Rinse off disinfectant with water.

After a spill:

- If the outside of a specimen container becomes contaminated, wash with disinfectant, e.g. sodium hypochlorite $0.1 \%(1 \mathrm{~g} / \mathrm{l})$ or similar disinfectant, then rinse with water.

- Immediately after any spills occur, wash the bench top with disinfectant e.g. sodium hypochlorite $1.0 \%$ ( $10 \mathrm{~g} / \mathrm{l})$ or similar disinfectant, wait at least 4 hours, then rinse off disinfectant with water.

When necessary, heat inactivation of HIV in semen collection vessels can be achieved by:

- Dry heat sterilization for at least 2 hours at $170{ }^{\circ} \mathrm{C}\left(340^{\circ} \mathrm{F}\right)$. Cover with foil before heating and allow to cool before handling.

- Steam sterilization (autoclaving) for at least 20 minutes at $121^{\circ} \mathrm{C}\left(250{ }^{\circ} \mathrm{F}\right)$ at $101 \mathrm{kPa}$ (15 psi or 1 atmosphere) above atmospheric pressure.

- Continuous boiling for 20-30 minutes. 


\section{A2.5 Safety precautions when handling liquid nitrogen}

- Liquid nitrogen is dangerous. Always handle it carefully, use only approved tanks and do not attempt to seal containers. Use tongs to withdraw objects immersed in liquid nitrogen.

- Protect eyes with a face shield or safety goggles. Protect hands with loosefitting dry leather or insulated gloves. Protect feet with closed shoes.

- When liquid nitrogen is spilled on a surface it tends to cover it completely, and therefore cools a large area. Objects that are soft and pliable at room temperature usually become hard and brittle at the temperature of liquid nitrogen.

- The extremely low temperature can cause serious injury. A spill on the skin can produce an effect similar to a burn. The gas issuing from the liquid is extremely cold. Delicate tissues, such as those of the eyes, can be damaged by even a brief exposure to the gas, which may not affect the skin of the face or hands.

- Stand clear of boiling and splashing liquid nitrogen, and its issuing cold gas. Boiling and splashing always occur when a warm container is charged, or when objects are inserted into the liquid. Always perform these operations slowly to minimize boiling and splashing.

- Avoid touching uninsulated pipes. Never allow any unprotected part of the body to touch pipes or vessels containing liquid nitrogen. The extremely cold metal may stick fast and the flesh will be torn when attempts are made to detach it.

- Work in well-ventilated areas. A small amount of liquid nitrogen forms a large amount of gas (at room temperature it is nine times its liquid volume). If nitrogen gas evaporates from the liquid in a closed room, the percentage of oxygen in the air may become low and create a risk of asphyxiation. Oxygen detectors, which trigger an alarm when the oxygen level falls below $17 \%(\mathrm{v} / \mathrm{v})$, are available and should be used where liquid nitrogen is stored.

- Use only tubes and straws especially made for freezing in liquid nitrogen. Care should always be taken because even these can explode as they become warm.

\section{Reference}

WHO (2004). Laboratory biosafety manual, 3rd ed. Geneva, World Health Organization (http://whqlibdoc.who.int/publications/2004/9241546506.pdf, last accessed 25 February 2010). 


\section{APPENDIX 3 Microscopy}

The best source of information for a particular microscope is the manufacturer's manual, which should include a diagram identifying all the parts. If such a manual is not available, it may be possible to obtain information on microscope set-up and use from an Internet search.

For the semen evaluations described in this manual, a phase-contrast microscope is recommended. The microscope, with at least a 50-watt light source, should preferably be binocular (have two eyepieces), with a phase condenser, and should be equipped with $\times 10, \times 20$ (or $\times 25$ ) and $\times 40$ (or $\times 63$ ) phase objectives (for general assessment, motility, vitality, and counting of spermatozoa and non-sperm cells), and a brightfield $\times 100$ oil-immersion objective (for assessment of morphology and vitality). A negative-phase lens may be needed for vitality measurements and some CASA equipment, and a fluorescence lens is required for fluorescence microscopy.

- The quality and price of objective lenses vary considerably (see Box A3.1). The more expensive objectives offer a better image, but lower-quality objectives may be adequate.

- Eyepiece reticles (reticules, graticules, eyepiece micrometers) are glass discs with scales of known dimensions, usually $5 \mathrm{~mm}$ or $10 \mathrm{~mm}$, or grids of various forms, inscribed on them. Some oculars have permanently mounted reticles; others can be unscrewed to allow a reticle to be inserted. They are available in different diameters, and should match exactly the diameter of the ocular. They may be calibrated with a stage micrometer to determine sperm dimensions. They are also used to limit the area of the field assessed for sperm motility. The one shown in Fig. 2.4(a) and Fig. A7.4(a) is a $5 \mathrm{~mm} \times 5 \mathrm{~mm}$ grid, which is a good size for motility assessment at both $\times 20$ and $\times 40$ magnification. Some technicians prefer this to a $10 \mathrm{~mm} \times 10 \mathrm{~mm}$ grid for estimating concentration or appraising morphology.

- A stage micrometer is a modified microscope slide with a scale etched on its surface, usually $1 \mathrm{~mm}$ divided into $10-\mu \mathrm{m}$ subdivisions. It can be used to calibrate the eyepiece micrometer or reticle grid, and to measure dimensions, e.g. for motility analyses (see Fig. A7.5).

The procedure described below will ensure the best possible image from the microscope. If the light pathway is properly aligned and adjusted, the image will be clear, crisp and unlikely to cause eye strain. The following procedures need to be performed when using a new microscope or whenever images are of poor quality.

\section{A3.1 Loading the sample}

- Place $10 \mu \mathrm{l}$ of semen (or other volume, see Box 2.4) on a microscope slide, cover with a $22 \mathrm{~mm} \times 22 \mathrm{~mm}$ coverslip (thickness number 1.5, $0.17 \mathrm{~mm}$ ) (or other dimension, see Box 2.4) and place the slide on the stage. You can also use a stage micrometer instead of a semen slide, to adjust the microscope.

- Turn on the light and adjust it to the intensity that gives maximum contrast while being comfortable for your eyes.

- Select the $\times 10$ positive-phase objective lens. Rotate the condenser wheel to correspond to the power of the chosen objective lens. 
Note: If the microscope is trinocular (i.e. has a third ocular to which a camera can be attached for photography or video-recording), there will be a light-deflection knob, which is generally located to the right of the eyepieces. This knob is likely to have three settings: one to allow all the light to go to the eyepieces, one to allow all light to go the camera, and a third that deflects half of the light to the eyepieces and half to the camera.

Box A3.1 The objective lens

Each microscope lens has information on it, such as:

$\begin{array}{lllll}\text { UPlanFl } & \text { PlanApo } & \text { Plan Neofluor } & \text { Plan } & \text { S Fluor } \\ 20 \times / 0.80 \mathrm{imm} \text { corr } & 40 \times / 0.75 \mathrm{Ph} 2 & 100 \times / 1.35 \text { oil iris } & 100 \times / 1.25 \text { oil Ph3 } & 20 \times / 0.75 \\ 160 / 0.17 & \infty / 0.17 & \infty /- & \infty / 0.17 \text { WD } 1.0 & \end{array}$

Explanations of the various markings are given below.

Plan: a planar lens, permitting a flat field of view, in which everything is in focus.

Apo: an apochromatic lens that is highly corrected for chromatic aberration.

F, FI, FL, Neofluor, Fluo, Fluotar, UV, S-Fluor: a lens that will transmit UV light and is used for fluorescence microscopy.

$100 \times, \times 63,40 \times$, etc.: the magnification of the lens.

$0.30,0.50,0.80,1.30,1.40$, etc.: the numerical aperture (NA) of the lens. This is an indication of the light-gathering ability of the lens. Together with the wavelength of the light used ( $\lambda$, lambda), the NA determines the resolution (the smallest distance between two objects that can be distinguished as separate). NA $=\eta \times \sin \alpha$, where $\eta$ (eta) is the refractive index of the immersion medium and $\alpha$ (alpha) is the angle between the edge of the cone of illumination and the vertical. As the maximum value of $\sin \alpha$ is 1.00 , the maximum NA is theoretically equal to $\eta$, but in practice the maximum value is 1.4 . Choose the highest NA for best resolution.

$\mathrm{Ph}, \mathrm{Ph} 1, \mathrm{Ph} 2, \mathrm{Ph} 3, \mathrm{NP}, \mathrm{N}$ : indicates a lens with a phase ring in it. $\mathrm{Ph}$ indicates positive-phase rings and NP or $\mathrm{N}$ negative-phase. Ph1, Ph2 and Ph3 lenses each require a different phase annulus in the condenser. Positive-phase-contrast optics permit intracellular structures to be seen (used for wet preparations and motility), while negative-phase-contrast optics produce white images against a dark background (used for wet preparation vitality or CASA).

Imm, immersion, oil, W: indicates a lens designed to work with a fluid-often oil, water (W) or glycerol-between the object and the lens to provide a sharper image. (If not indicated, the lens is "dry" and should not be used with a liquid.)

Iris: indicates a lens with an iris controlled by a knurled ring.

Corr: indicates a lens with a knurled correction collar that allows the use of immersion media of different refractive indices.

$160, \infty$ : the tube length or distance between the eyepiece and the objective. This is usually $160 \mathrm{~mm}$ but in modern lenses can be infinity $(\infty)$.

0.17 , - : the thickness of the coverslip required for the objective. Coverslip number 1.5 (thickness $0.16-0.19 \mathrm{~mm}$ ) is useful for most purposes. Haemocytometers need coverslips number 4 (thickness $0.44 \mathrm{~mm}$ ). "-" means that the thickness of the coverslip is not important or that immersion fluid can be added directly to the slide.

WD: working distance; the distance from the front lens element of the objective to the closest surface of the coverslip when the specimen is in sharp focus. The WD generally decreases as the magnification and NA increase, giving rise to lenses with working distances that are normal (NWD, up to $5 \mathrm{~mm}$ ), long (LWD, 5.25-9.75 mm), extra-long (ELWD, 10-14 mm) and super-long (SLWD, 15-30 mm). Some microscopes may require an LWD lens for use with an improved Neubauer chamber.

Refractive index: the extent of phase retardation of light as it passes through a medium. The refractive index (RI, $\eta$, eta) of a vacuum is 1.0000 , of air is approximately $1.0(1.0008)$, of water is 1.33 , of glycerol is 1.47 and of most immersion oils is 1.515. Mounting media after drying have similar refractive indices (1.488-1.55) to that of glass (1.50-1.58). 


\section{A3.2 Adjusting the oculars}

- Adjust the space between the oculars (eyepieces) to your own eyes by pulling the oculars apart or pushing them together.

\section{A3.3 Focusing the image}

- Rotate the coarse focus adjustment to bring the stage as close to the $\times 20$ or $\times 40$ objective as possible. To avoid breaking the objective lens and the slide, look at the objective and stage from the front or side, not through the eyepieces. Use the coarse focus to adjust the height of the stage so that the slide is almost in contact with the objective. Note which way the coarse focus has to be turned to lower the stage away from the objective.

- Looking through both eyepieces, slowly turn the coarse focus adjustment to move the stage away from the objective gradually, until the specimen is in approximate focus. Use the fine-adjustment knob to achieve the best focus.

Note: If focus is hard to find, try focusing on the ground-glass ends of a slide to get close to the correct focal plane.

\section{A3.4 Focusing the oculars}

- With some microscopes, the two oculars can be focused independently. With others, one ocular is fixed and the other can be focused.

- Adjustable oculars are usually marked with a "+/ 0 / -" scale. Adjust the ocular to " 0 " before beginning this process.

- If one ocular is fixed, look through the fixed ocular only (close or cover your other eye).

- Focus the specimen image using the fine-focus adjustment. It is helpful to focus on a non-moving object, e.g. a dead spermatozoon, dust particle or stage micrometer grid.

- Focus the adjustable ocular by looking through it and closing or covering the eye over the fixed ocular. Rotate the knurled ring at the base of the eyepiece to "+" or "-" until the focus is appropriate for your eye.

\section{A3.5 Foc using the light condenser}

- Close down the field diaphragm (over the source of light at the base of the microscope).

- Raise or lower the condenser using the small knobs on the left or right of the condenser unit until the edges of the diaphragm are in the sharpest focus possible, and the circle of light is small and clear. This position will generally be achieved when the condenser is in the top-most position. The edge of the light image may change from blue to red as the condenser is focused (chromatic aberration), and the edges of the condenser will remain slightly blurred. The light may or may not be centred. 
Note: If the field aperture has no iris diaphragm, focus on a sharp object (e.g. a pencil point) placed on the light source.

\section{A3.6 Centring the condenser}

- Centre the field diaphragm with the condenser centring knobs. These are generally two (usually knurled) knobs coming out diagonally from the front or side underneath the condenser.

- Once the light image is centred, open the field diaphragm so that the light just fills the field of view. Do not open the field diaphragm beyond that point.

- Close the condenser aperture until the glare disappears.

Note: Directly behind the right-hand condenser centring screw, there may be small screws that lock the condenser in place. Be careful not to turn them when centring the condenser, as loosening them will allow the entire condenser to be removed from the microscope.

\section{A3.7 Adjusting the phase rings}

- This is done with the use of a centring telescope, available from the microscope manufacturer.

- Bring into view the appropriate phase annulus in the condenser for the objective being used.

- Remove one eyepiece and replace it with the centring telescope. Focus the ring of the centring telescope by holding the base of it with one hand and rotating the top portion with the other hand while looking through it. Turn it until the two rings are in sharp focus: one ring is dark (phase annulus) and one light (light annulus).

- Align these rings so that they are concentric by turning the phase-adjustment knobs located on the phase condenser. These knobs are usually located towards the back of the condenser.

- Replace the centring telescope with the microscope ocular.

\section{A3.8 Fluorescence microscopy}

Fluorescence microscopy is used to detect the nuclei of spermatozoa in the sensitive counting procedure using Hoechst 33342 dye (see Section 2.11.2) and the acrosome reaction using FITC-labelled lectin (see Section 4.4.1). The excitation spectral maxima of Hoechst 33342 dye and FITC are $346 \mathrm{~nm}$ and $494 \mathrm{~nm}$, respectively, and the corresponding emission maxima are $460 \mathrm{~nm}$ and $520 \mathrm{~nm}$. A fluorescence lens is required (see Box A3.1). Each model of microscope will have, as optional equipment for purchase, the requisite set of dichroic mirrors and barrier filters needed to examine these dyes. 


\section{APPENDIX $4 \quad$ Stock solutions}

For all solutions, a supply of purified water (distilled, double-distilled or deionized) is required.

\section{A4.1 Biggers, Whitten and Whittingham}

BWW stock solution (Biggers et al., 1971)

1. To $1000 \mathrm{ml}$ of purified water add $5.54 \mathrm{~g}$ of sodium chloride $(\mathrm{NaCl}), 0.356 \mathrm{~g}$ of potassium chloride ( $\mathrm{KCl}), 0.294 \mathrm{~g}$ of magnesium sulfate heptahydrate $\left(\mathrm{MgSO}_{4} \cdot 7 \mathrm{H}_{2} \mathrm{O}\right), 0.250 \mathrm{~g}$ of calcium chloride dihydrate $\left(\mathrm{CaCl}_{2} \cdot 2 \mathrm{H}_{2} \mathrm{O}\right)$ and $0.162 \mathrm{~g}$ of potassium dihydrogen phosphate $\left(\mathrm{KH}_{2} \mathrm{PO}_{4}\right)$.

2. Adjust the $\mathrm{pH}$ to 7.4 with $1 \mathrm{~mol} / /$ sodium hydroxide $(\mathrm{NaOH})$.

3. Add $1.0 \mathrm{ml}(0.04 \%, 0.4 \mathrm{~g} / \mathrm{l})$ phenol red per litre.

Note: This solution can be stored for several weeks at $4{ }^{\circ} \mathrm{C}$.

BWW working solution.

On the day of use:

1. Supplement $100 \mathrm{ml}$ of stock solution with $210 \mathrm{mg}$ of sodium bicarbonate $(\mathrm{NaH}$ $\left.\mathrm{CO}_{3}\right), 100 \mathrm{mg}$ of $\mathrm{D}$-glucose, $0.37 \mathrm{ml}$ of $60 \%(\mathrm{v} / \mathrm{v})$ sodium lactate syrup, $3 \mathrm{mg}$ of sodium pyruvate, $350 \mathrm{mg}$ of fraction $\mathrm{V}$ bovine serum albumin, 10000 units of penicillin and $10 \mathrm{mg}$ of streptomycin sulfate.

2. Warm to $37^{\circ} \mathrm{C}$ before use in an atmosphere of $5 \%(\mathrm{v} / \mathrm{v}) \mathrm{CO}_{2}, 95 \%(\mathrm{v} / \mathrm{v})$ air.

Note 1: For incubation in air: add $20 \mathrm{mmol} / \mathrm{l}$ Hepes (Na salt: $5.21 \mathrm{~g} / \mathrm{l})$ and reduce $\mathrm{NaHCO}_{3}$ to $0.366 \mathrm{~g} / \mathrm{l}$.

Note 2: For density gradients (see Section 5.5.1): prepare a 10x concentrated stock solution by using 10 times the specified weights of the compounds, except for the phenol red. After preparing the gradient, supplement $100 \mathrm{ml}$ as above.

\section{A4.2 Dulbecco's phosphate-buffered saline}

1. Dulbecco's glucose-PBS: to $750 \mathrm{ml}$ of purified water add $0.2 \mathrm{~g}$ of potassium chloride $(\mathrm{KCl}), 0.2 \mathrm{~g}$ of potassium dihydrogen phosphate $\left(\mathrm{KH}_{2} \mathrm{PO}_{4}\right), 0.1 \mathrm{~g}$ of magnesium chloride hexahydrate $\left(\mathrm{M} \mathrm{gCl}_{2} \cdot 6 \mathrm{H}_{2} \mathrm{O}\right), 8.0 \mathrm{~g}$ of sodium chloride $(\mathrm{NaCl}), 2.16 \mathrm{~g}$ of disodium hydrogen phosphate heptahydrate $\left(\mathrm{Na}_{2} \mathrm{HPO}_{4} \cdot 7 \mathrm{H}_{2} \mathrm{O}\right)$ and $1.00 \mathrm{~g}$ of D-glucose.

2. Dissolve $0.132 \mathrm{~g}$ of calcium chloride dihydrate $\left(\mathrm{CaCl}_{2} \cdot 2 \mathrm{H}_{2} \mathrm{O}\right)$ in $10 \mathrm{ml}$ of purified water and add slowly to the above solution with stirring.

3. Adjust the $\mathrm{pH}$ to 7.4 with $1 \mathrm{~mol} / /$ sodium hydroxide $(\mathrm{NaOH})$.

4. Make up to $1000 \mathrm{ml}$ with purified water. 
Note 1: To prevent precipitation, add $\mathrm{CaCl}_{2}$ separately, slowly and with stirring.

Note 2: If required, add $0.3 \mathrm{~g}$ of bovine serum albumin (BSA) (essential fatty acid free) per $100 \mathrm{ml}$ before use.

\section{A4.3 Earle's medium}

1. To $750 \mathrm{ml}$ of purified water add $6.8 \mathrm{~g}$ of sodium chloride $(\mathrm{NaCl}), 2.2 \mathrm{~g}$ of sodium bicarbonate $\left(\mathrm{NaHCO}_{3}\right), 0.14 \mathrm{~g}$ of sodium dihydrogen phosphate monohydrate $\left(\mathrm{NaH}_{2} \mathrm{PO}_{4} \cdot \mathrm{H}_{2} \mathrm{O}\right), 0.4 \mathrm{~g}$ of potassium chloride $(\mathrm{KCl}), 0.20 \mathrm{~g}$ of magnesium sulfate heptahydrate $\left(\mathrm{MgSO}_{4} \cdot 7 \mathrm{H}_{2} \mathrm{O}\right)$ and $1.0 \mathrm{~g}$ of D-glucose.

2. Dissolve $0.20 \mathrm{~g}$ of anhydrous calcium chloride $\left(\mathrm{CaCl}_{2}\right)$ slowly in the above solution with stirring.

3. Adjust the $\mathrm{pH}$ to 7.4 with $1 \mathrm{~mol} / \mathrm{l}$ hydrochloric acid $(\mathrm{HCl})$ or $1 \mathrm{~mol} / \mathrm{l}$ sodium hydroxide $(\mathrm{NaOH})$.

4. Make up to $1000 \mathrm{ml}$ with purified water.

Note 1: For incubation in air: add $20 \mathrm{mmol} / \mathrm{l}$ Hepes ( $\mathrm{Na}$ salt: $5.21 \mathrm{~g} / \mathrm{l}$ ) and reduce $\mathrm{NaHCO}_{3}$ to $0.366 \mathrm{~g} / \mathrm{l}$.

Note 2: For density gradients (see Section 5.5.1): prepare a 10x concentrated stock solution by using 10 times the specified weights of the compounds, except for the bicarbonate. After preparing the gradient, supplement $100 \mathrm{ml}$ with $0.22 \mathrm{~g}$ of $\mathrm{NaHCO}_{3}$.

\section{A4.4 Ham's F-10 medium}

1. To $750 \mathrm{ml}$ of purified water add $7.4 \mathrm{~g}$ of sodium chloride $(\mathrm{NaCl}), 1.2 \mathrm{~g}$ of sodium bicarbonate $\left(\mathrm{NaHCO}_{3}\right), 0.285 \mathrm{~g}$ of potassium chloride $(\mathrm{KCl}), 0.154 \mathrm{~g}$ of sodium monosodium phosphate $\left(\mathrm{Na}_{2} \mathrm{HPO}_{4}\right), 0.153 \mathrm{~g}$ of magnesium sulfate heptahydrate $\left(\mathrm{MgSO}_{4} \cdot 7 \mathrm{H}_{2} \mathrm{O}\right), 0.083 \mathrm{~g}$ of potassium dihydrogen phosphate $\left(\mathrm{KH}_{2} \mathrm{PO}_{4}\right), 0.044 \mathrm{~g}$ of calcium chloride dihydrate $\left(\mathrm{CaCl}_{2} \cdot 2 \mathrm{H}_{2} \mathrm{O}\right)$ and $1.1 \mathrm{~g}$ of D-glucose.

2. Adjust the $\mathrm{pH}$ to 7.4 with $1 \mathrm{~mol} / /$ sodium hydroxide $(\mathrm{NaOH})$.

3. Make up to $1000 \mathrm{ml}$ with purified water.

Note 1: For incubation in air: add $20 \mathrm{mmol} / \mathrm{l}$ Hepes ( $\mathrm{Na}$ salt: $5.21 \mathrm{~g} / \mathrm{l}$ ) and reduce $\mathrm{NaHCO}_{3}$ to $0.366 \mathrm{~g} / \mathrm{l}$.

Note 2: For density gradients (see Section 5.5.1): prepare a 10x concentrated stock solution by increasing the weights of the compounds 10 -fold, except for the bicarbonate. After preparing the gradient, supplement $100 \mathrm{ml}$ with $0.12 \mathrm{~g} \mathrm{NaHCO}_{3}$. 


\section{A4.5 Hanks' balanced salt solution}

1. To $750 \mathrm{ml}$ of purified water add $8.0 \mathrm{~g}$ of sodium chloride $(\mathrm{NaCl}), 0.4 \mathrm{~g}$ of potassium chloride $(\mathrm{KCl}), 0.35 \mathrm{~g}$ of sodium bicarbonate $\left(\mathrm{NaHCO}_{3}\right), 0.185 \mathrm{~g}$ of calcium chloride dihydrate $\left(\mathrm{CaCl}_{2} \cdot 2 \mathrm{H}_{2} \mathrm{O}\right), 0.1 \mathrm{~g}$ of magnesium chloride hexahydrate $\left(\mathrm{M} \mathrm{gCl}_{2} \cdot 6 \mathrm{H}_{2} \mathrm{O}\right), 0.1 \mathrm{~g}$ of magnesium sulfate heptahydrate $\left(\mathrm{MgSO}_{4} \cdot 7 \mathrm{H}_{2} \mathrm{O}\right), 0.06 \mathrm{~g}$ of potassium dihydrogen phosphate $\left(\mathrm{KH}_{2} \mathrm{PO}_{4}\right), 0.048 \mathrm{~g}$ of sodium dihydrogen phosphate $\left(\mathrm{NaH}_{2} \mathrm{PO}_{4}\right)$ and $1.0 \mathrm{~g}$ of D-glucose.

2. Adjust the $\mathrm{pH}$ to 7.4 with $1 \mathrm{~mol} / /$ sodium hydroxide $(\mathrm{NaOH})$.

3. Make up to $1000 \mathrm{ml}$ with purified water.

\section{A4.6 Human tubal fluid}

Original formulation (Quinn et al., 1985):

1. To $750 \mathrm{ml}$ of purified water add $5.931 \mathrm{~g}$ of sodium chloride $(\mathrm{NaCl}), 0.35 \mathrm{~g}$ of potassium chloride $(\mathrm{KCl}), 0.05 \mathrm{~g}$ of magnesium sulfate heptahydrate $\left(\mathrm{MgSO}_{4} \cdot 7 \mathrm{H}_{2} \mathrm{O}\right), 0.05 \mathrm{~g}$ of potassium dihydrogen phosphate $\left(\mathrm{KH}_{2} \mathrm{PO}_{4}\right), 2.1 \mathrm{~g}$ of sodium bicarbonate $\left(\mathrm{NaHCO}_{3}\right), 0.5 \mathrm{~g}$ of $\mathrm{D}$-glucose, $0.036 \mathrm{~g}$ of sodium pyruvate, $0.3 \mathrm{~g}$ of calcium chloride dihydrate $\left(\mathrm{CaCl}_{2} \cdot 2 \mathrm{H}_{2} \mathrm{O}\right)$ and $4.0 \mathrm{~g}$ of sodium DL-lactate (60\% (v/v) syrup).

2. To $1 \mathrm{ml}$ of the above medium add $10 \mu \mathrm{g}$ phenol red, $100 \mathrm{U}$ penicillin and $50 \mu \mathrm{g}$ streptomycin sulfate

3. Adjust the $\mathrm{pH}$ to 7.4 with $1 \mathrm{~mol} / \mathrm{l}$ hydrochloric acid $(\mathrm{HCl})$.

4. Make up to $1000 \mathrm{ml}$ with purified water.

Note 1: For incubation in air: add $20 \mathrm{mmol} / \mathrm{l}$ Hepes ( $\mathrm{Na}$ salt: $5.21 \mathrm{~g} / \mathrm{l}$ ) and reduce $\mathrm{NaHCO}_{3}$ to $0.366 \mathrm{~g} / \mathrm{l}$.

Note 2: For density gradients (see Section 5.5.1): prepare a 10x concentrated stock solution by using 10 times the specified weights of the compounds, except for the bicarbonate, pyruvate and lactate. After preparing the gradient, supplement $100 \mathrm{ml}$ with $0.21 \mathrm{~g}$ of $\mathrm{NaHCO}_{3}, 0.0036 \mathrm{~g}$ of sodium pyruvate and $0.4 \mathrm{~g}$ of sodium lactate.

\section{A4.7 Krebs-Ringer medium}

Krebs-Ringer medium (KRM) without phenol red:

1. To $750 \mathrm{ml}$ of purified water add $6.9 \mathrm{~g}$ of sodium chloride $(\mathrm{NaCl}), 2.1 \mathrm{~g}$ of sodium bicarbonate $\left(\mathrm{NaHCO}_{3}\right), 0.35 \mathrm{~g}$ of potassium chloride $(\mathrm{KCl}), 0.32 \mathrm{~g}$ of calcium chloride dihydrate $\left(\mathrm{CaCl}_{2} \cdot 2 \mathrm{H}_{2} \mathrm{O}\right), 0.18 \mathrm{~g}$ of sodium dihydrogen phosphate dihydrate $\left(\mathrm{NaH}_{2} \mathrm{PO}_{4} \cdot 2 \mathrm{H}_{2} \mathrm{O}\right), 0.1 \mathrm{~g}$ of magnesium chloride hexahydrate $\left(\mathrm{MgCl}_{2} \cdot 6 \mathrm{H}_{2} \mathrm{O}\right)$ and $0.9 \mathrm{~g}$ of $\mathrm{D}$-glucose.

2. Adjust the $\mathrm{pH}$ to 7.4 with $1 \mathrm{~mol} / /$ sodium hydroxide $(\mathrm{NaOH})$.

3. Make up to $1000 \mathrm{ml}$ with purified water. 


\section{A4.8 Tris-buffered saline}

1. To $750 \mathrm{ml}$ of purified water add $6.055 \mathrm{~g}$ of Tris base and $8.52 \mathrm{~g}$ of sodium chloride $(\mathrm{NaCl})$.

2. Adjust the $\mathrm{pH}$ to 8.2 with $1 \mathrm{~mol} / /$ hydrochloric acid $(\mathrm{HCl})$.

3. Make up to $1000 \mathrm{ml}$ with purified water.

Note: A 10x concentrated stock solution can be made by using 10 times the specified weights of the compounds. For use, dilute 10-fold with purified water and adjust the $\mathrm{pH}$ with $1 \mathrm{~mol} / \mathrm{l} \mathrm{HCl}$.

\section{A4.9 Tyrode's solution}

1. To $750 \mathrm{ml}$ of purified water add $0.2 \mathrm{~g}$ of anhydrous calcium chloride $\left(\mathrm{CaCl}_{2}\right)$, $0.2 \mathrm{~g}$ of potassium chloride $(\mathrm{KCl}), 0.05 \mathrm{~g}$ of disodium hydrogen phosphate $\left(\mathrm{Na}_{2} \mathrm{HPO}_{4}\right), 0.2 \mathrm{~g}$ of magnesium chloride hexahydrate $\left(\mathrm{M} \mathrm{gCl}_{2} \cdot 6 \mathrm{H}_{2} \mathrm{O}\right), 8.0 \mathrm{~g}$ of sodium chloride $(\mathrm{NaCl}), 1.0 \mathrm{~g}$ of sodium bicarbonate $\left(\mathrm{NaHCO}_{3}\right)$ and $1.0 \mathrm{~g}$ of D-glucose.

2. Adjust the $\mathrm{pH}$ to 7.4 with $1 \mathrm{~mol} / \mathrm{l}$ hydrochloric acid $(\mathrm{HCl})$ or $1 \mathrm{~mol} / /$ sodium hydroxide $(\mathrm{NaOH})$.

3. Make up to $1000 \mathrm{ml}$ with purified water.

4. If required, add $0.3 \mathrm{~g}$ of BSA (essential fatty acid free) per $100 \mathrm{ml}$ before use.

\section{A4.10 Papanic olaou stain}

Commercially available stains are usually satisfactory, but the stain can be prepared in the laboratory.

Note: Check the acidity of the purified water before preparing the different grades of ethanol. The $\mathrm{pH}$ should be 7.0.

EA-36 (equivalent to EA-50)

Constituents

1. Eosin $Y$ (colour index 45380)

$10 \mathrm{~g}$

2. Bismarck brown Y (colour index 21000)

$10 \mathrm{~g}$

3. Light-green SF, yellowish (colour index 42095)

$10 \mathrm{~g}$

4. Purified water

$300 \mathrm{ml}$

5. Ethanol 95\% (v/v)

$2000 \mathrm{ml}$

6. Phosphotungstic acid

$4 \mathrm{~g}$

7. Saturated aqueous lithium carbonate $(>1.3 \mathrm{~g} / 100 \mathrm{ml})$

$0.5 \mathrm{ml}$ 
Stock solutions

Prepare separate $10 \%(100 \mathrm{~g} / \mathrm{l})$ solutions of each of the stains as follows:

1. Dissolve $10 \mathrm{~g}$ of eosin $\mathrm{Y}$ in $100 \mathrm{ml}$ of purified water.

2. Dissolve $10 \mathrm{~g}$ of Bismarck brown $\mathrm{Y}$ in $100 \mathrm{ml}$ of purified water.

3. Dissolve $10 \mathrm{~g}$ of light-green SF in $100 \mathrm{ml}$ of purified water.

Preparation

1. To prepare 2 litres of stain, $\operatorname{mix} 50 \mathrm{ml}$ of eosin $Y$ stock solution with $10 \mathrm{ml}$ of the Bismarck brown $Y$ stock solution and add $12.5 \mathrm{ml}$ of light-green SF stock solution.

2. Make up to $2000 \mathrm{ml}$ with $95 \%$ (v/v) ethanol.

3. Add $4 \mathrm{~g}$ of phosphotungstic acid.

4. Add $0.5 \mathrm{ml}$ of saturated lithium carbonate solution.

5. Mix well and store at room temperature in dark-brown tightly capped bottles.

Note 1: The solution is stable for 2-3 months.

Note 2: Pass through a $0.45-\mu \mathrm{m}$ filter before use.

Orange G6

Constituents

1. Orange $\mathrm{G}$ crystals (colour index 16230) $10 \mathrm{~g}$

2. Purified water $100 \mathrm{ml}$

3. $95 \%(v / v)$ ethanol $\quad 1000 \mathrm{ml}$

4. Phosphotungstic acid $\quad 0.15 \mathrm{~g}$

Stock solution number 1 (orange G6, 10\% (100 g/l) solution)

1. Dissolve $10 \mathrm{~g}$ of Orange $\mathrm{G}$ crystals in $100 \mathrm{ml}$ of purified water.

2. Shake well. Allow to stand in a dark-brown or aluminium-foil-covered stoppered bottle at room temperature for 1 week before using.

Stock solution number 2 (orange $\mathrm{G} 6,0.5 \%$ solution)

1. To $50 \mathrm{ml}$ of stock solution number 1 add $950 \mathrm{ml}$ of $95 \%(\mathrm{v} / \mathrm{v})$ ethanol.

2. Add $0.15 \mathrm{~g}$ of phosphotungstic acid.

3. Mix well. Store in dark-brown or aluminium-foil-covered stoppered bottles at room temperature. 
Note 1: Filter before use.

Note 2: The solution is stable for 2-3 months.

Harris's haematoxylin without acetic acid

Constituents

1. Haematoxylin (dark crystals; colour index 75290)

2. Ethanol $95 \%(\mathrm{v} / \mathrm{v})$

3. Aluminium ammonium sulfate dodecahydrate $\left(\mathrm{AINH}_{4}\left(\mathrm{SO}_{4}\right)_{2} \cdot 12 \mathrm{H}_{2} \mathrm{O}\right)$

4. Mercuric oxide $(\mathrm{HgO})$

Preparation

1. Dissolve $160 \mathrm{~g}$ of aluminium ammonium sulfate dodecahydrate in $1600 \mathrm{ml}$ of purified water by heating.

2. Dissolve $8 \mathrm{~g}$ of haematoxylin crystals in $80 \mathrm{ml}$ of $95 \%(\mathrm{v} / \mathrm{v})$ ethanol.

3. Add the haematoxylin solution to the aluminium ammonium sulfate solution.

4. Heat the mixture to $95{ }^{\circ} \mathrm{C}$.

5. Remove the mixture from the heat and slowly add $6 \mathrm{~g}$ of mercuric oxide while stirring.

Note: The solution will be dark purple in colour.

6. Immediately plunge the container into a cold waterbath.

7. When the solution is cold, filter.

8. Store in dark-brown or aluminium-foil-covered bottles at room temperature.

9. Allow to stand for 48 hours before using.

10. Dilute the required amount with an equal amount of purified water.

11. Filter again. 
Scott's tap water substitute solution

Note: Scott's solution is used only when the ordinary tap water is insufficient to return blue color to the nucleus; it should be changed frequently, e.g. after rinsing 20 to 25 slides.

\section{Constituents}

1. Sodium bicarbonate $\left(\mathrm{NaHCO}_{3}\right) 3.5 \mathrm{~g}$

2. Magnesium sulphate heptahydrate $\left(\mathrm{MgSO}_{4} \cdot 7 \mathrm{H}_{2} \mathrm{O}\right) 20.0 \mathrm{~g}$

3. Several crystals of thymol (if required as preservative)

4. Purified water $1000 \mathrm{ml}$

Acid ethanol solution

Constituents

1. Ethanol $99.5 \%(\mathrm{v} / \mathrm{v}) 300 \mathrm{ml}$

2. Concentrated hydrochloric acid $(\mathrm{HCl}) 2.0 \mathrm{ml}$

3. Purified water $100 \mathrm{ml}$

\section{References}

Biggers J D et al. (1971). The culture of mouse embryos in vitro. In: Daniel J C, ed. Methods in mammalian embryology. San Francisco, WH Freeman: 86-116.

Quinn P et al. (1985). Improved pregnancy rate in human in-vitro fertilization with the use of a medium based on the composition of human tubal fluid. Fertility and Sterility, 44:493-498. 


\section{APPENDIX 5 Cervical mucus}

\section{A5.1 Introduction}

Spermatozoa within cervical mucus are suspended in a fluid medium. The interaction of spermatozoa with the secretions of the female reproductive tract is of critical importance for their survival and functioning. There is at present no practical method of evaluating the effects of human uterine and tubal fluids on spermatozoa. However, cervical mucus is readily available for sampling and study.

The epithelium of the human cervix comprises different types of secretory cells, and the nature and abundance of secretory granules vary in different parts of the cervix. Secretions from these cells contribute to the cervical mucus. Ovarian hormones regulate the secretion of cervical mucus: $17 \beta$-estradiol stimulates the production of copious amounts of watery mucus and progesterone inhibits the secretory activity of the epithelial cells. The amount of cervical mucus secreted shows cyclical variations. In women of reproductive age with a normal menstrual cycle, the daily mucus production varies from $500 \mu \mathrm{l}$ at mid-cycle to less than $100 \mu \mathrm{l}$ at other times. Small amounts of endometrial, tubal and possibly follicular fluids may also contribute to the cervical mucus pool. In addition, leukocytes and cellular debris from the uterine and cervical epithelia are present.

Cervical mucus is a heterogeneous secretion containing over $90 \%$ water. It exhibits a number of rheological properties:

- Viscosity (consistency) is influenced by the molecular arrangement and by the protein and ionic concentrations of the cervical mucus. Mucus varies during the cycle from highly viscous (often cellular) just before menstruation to watery at mid-cycle just before ovulation. By the time ovulation is completed, the viscosity of the mucus has already begun to increase again.

- Spinnbarkeit is the term used to describe the fibrosity, the "threadability", or the elasticity characteristics of cervical mucus.

- Ferning refers to the degree and pattern of crystallization observed when cervical mucus is dried on a glass surface (see Fig. A5.1).

Cervical mucus is a hydrogel comprising a high-viscosity component and a lowviscosity component made up of electrolytes, organic compounds and soluble proteins. The high-viscosity component is a macromolecular network of mucin, which influences the rheological properties of the mucus. Cervical mucin is a fibrillar system consisting of subunits made of a peptide core and oligosaccharide side-chains. Cyclical alteration in the constituents of cervical mucus influences the ability of spermatozoa to penetrate and survive. Spermatozoa can penetrate human cervical mucus from approximately the ninth day of a normal 28-day cycle; penetrability increases gradually to reach a peak just before ovulation. Sperm penetration then begins to diminish before large changes in mucus properties are apparent. Individual variations in timing and degree of sperm penetrability are common. Motile spermatozoa may be guided by strands of cervical mucus to the cervical crypts, where they may be retained and released slowly into the uterus and Fallopian tubes. 
Fig. A5.1 Examples of fern formation in cervical mucus air-dried on a glass slide

(a) Ferning: 1, primary stem; 2, secondary stem; 3, tertiary stem; 4, quaternary stem (score 3);

(b) mainly primary and secondary stems (score 2 ) but some tertiary stems also present; (c) atypical fern crystallization (score 1); (d) no crystallization (score 0 ). The round structures are air bubbles. See section A5.3.3 for explanation of scoring.
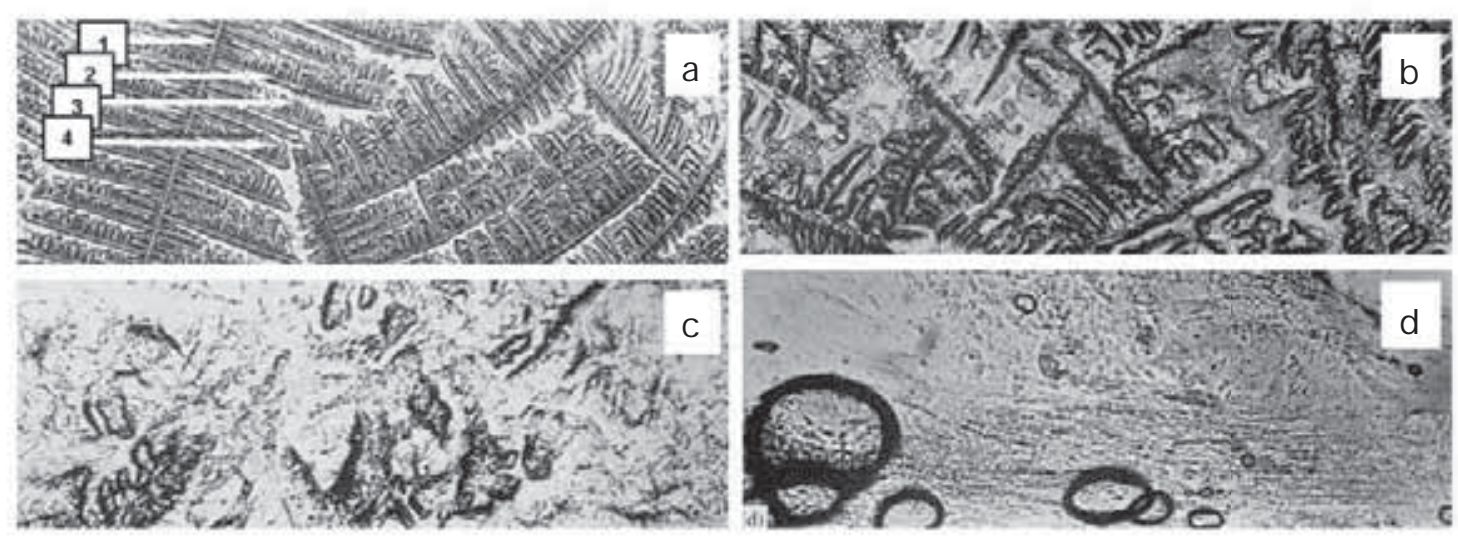

Comment: It is important to evaluate sperm-cervical mucus interaction as part of any complete investigation of infertility. A finding of abnormal sperm-cervical mucus interaction may be an indication for artificial insemination or other forms of assisted reproduction.

\section{A5.2 Collection and preservation of cervical mucus}

\section{A5.2.1 Collection procedure}

Expose the cervix with a speculum and gently wipe the external os with a cotton swab to remove the external pool of vaginal contaminants. Remove the exocervical mucus with the swab or with forceps. Collect cervical mucus from the endocervical canal by aspiration with a mucus syringe, tuberculin syringe (without needle), pipette or polyethylene tube. The manner in which suction pressure is applied to the collection device should be standardized. Advance the tip of the device approximately $1 \mathrm{~cm}$ into the cervical canal before applying suction. Then maintain suction as the device is withdrawn. J ust before the device is completely withdrawn from the external cervical os, release the suction pressure. It is then advisable to clamp the catheter to protect against accumulation of air bubbles or vaginal material in the collected mucus when the device is removed from the cervical canal. Whenever possible, the quality of the mucus should be evaluated immediately on collection. If this is not possible, the mucus should be preserved (see Section A5.2.2) until it can be tested. 
When cervical mucus is to be collected other than at mid-cycle, its production can be increased by the administration of $20-80 \mu \mathrm{g}$ of ethinyl estradiol each day for 7-10 days before collection. This procedure will produce a more hydrated, and therefore less viscous, mucus secretion (Eggert-Kruse et al., 1989). While this approach may be useful in assessing sperm-mucus interaction in vitro, it will not necessarily reflect the in-vivo situation for the couple when hormones are not administered.

\section{A5.2.2 Storage and preservation}

Mucus can be preserved either in the original collection device or in small testtubes sealed with a stopper or with self-sealing laboratory film to avoid dehydration. Care should be taken to minimize the air space in the storage container. The samples should be preserved in a refrigerator at $4{ }^{\circ} \mathrm{C}$ for up to 5 days. If possible, mucus specimens should be used within 2 days of collection; the interval between collection and use should always be noted. Rheological and sperm penetration tests should not be performed on mucus specimens that have been frozen and thawed.

\section{A5.3 Evaluation of cervical mucus}

Evaluation of the properties of cervical mucus includes assessment of spinnbarkeit, ferning (crystallization), viscosity and $\mathrm{pH}$. Appendix 6 contains a sample form for scoring and recording these cervical mucus properties according to the system devised by Moghissi (1976), based on an original proposal by Insler et al. (1972). The score is derived from the volume of cervical mucus collected (see Section A5.3.1) and the four variables (see Sections A5.3.2 to A5.3.5) describing its characteristics and appearance. The $\mathrm{pH}$ of the mucus is not included in the total cervical mucus score, but should be measured as an important determinant of sperm-mucus interaction (Eggert-Kruse et al., 1993). The maximum score is 15. A score greater than 10 is usually indicative of good cervical mucus favouring sperm penetration; a score of less than 10 may mean that the cervical mucus is unfavourable to sperm penetration.

\section{A5.3.1 Volume}

The viscosity of mucus makes accurate measurement of volume difficult. It can be estimated from the length of the mucus within catheter tubing of known diameter (see Box A5.1).

\section{Box A5.1 Determining the volume of mucus collected}

The volume of a mucus preparation $\left(V, \mu l=\mathrm{mm}^{3}\right)$ is obtained by multiplying the cross-sectional area of the tubing $\left(A, \mathrm{~mm}^{2}\right)$ by the length $(L, \mathrm{~mm})$ containing mucus: $\mathrm{V}=\mathrm{A} \times \mathrm{L}$. The cross-sectional area $\mathrm{A}=\pi \mathrm{r}^{2}$, where $\pi$ is approximately 3.142 and $r$ is the radius of the tubing. Thus a $10 \mathrm{~cm}(100 \mathrm{~mm})$ length of mucus in $2 \mathrm{~mm}$ diameter tubing $\left(A=3.142 \times 1 \times 1=3.142 \mathrm{~mm}^{2}\right)$ has a volume of $A \times L=3.142 \times 100=314 \mathrm{~mm}^{3}$ $=314 \mu \mathrm{l}$ or $0.31 \mathrm{ml}$. 
Volume is scored as follows:

$$
\begin{aligned}
& 0=0 \mathrm{ml} \\
& 1=0.01-0.10 \mathrm{ml} \text { or approximately } 0.1 \mathrm{ml} \\
& 2=0.11-0.29 \mathrm{ml} \text { or approximtely } 0.2 \mathrm{ml} \\
& 3=>0.3 \mathrm{ml} \text { or approximately } 0.3 \mathrm{ml} \text { or more }
\end{aligned}
$$

\section{A5.3.2 Viscosity (consistency)}

The viscosity of cervical mucus is the most important factor influencing sperm penetration. There is little resistance to sperm migration through the cervical mucus in mid-cycle, but viscous mucus-such as that observed during the luteal phase-forms a more formidable barrier.

Viscosity is scored as follows:

$$
\begin{aligned}
& 0=\text { thick, highly viscous, premenstrual mucus } \\
& 1=\text { mucus of intermediate viscosity } \\
& 2=\text { mildly viscous mucus } \\
& 3=\text { watery, minimally viscous, mid-cycle (preovulatory) mucus }
\end{aligned}
$$

\section{A5.3.3 Ferning}

Ferning (see Fig. A5.1) is scored by examination of cervical mucus that has been air-dried on glass microscope slides. Such preparations reveal various patterns of crystallization, which may have a fern-like appearance. Depending on the composition of the mucus, the "ferns" may have only a primary stem, or the stem may branch once, twice or three times to produce secondary, tertiary and quaternary stems. Several fields around the preparation should be observed, and the score expressed as the highest degree of ferning that is typical of the specimen.

Fern types can be very variable, depending on, for example, the thickness of the preparation and the number of cells present. A preparation may display more than one stage of ferning: sometimes all stages can be found in one preparation.

Ferning is scored as follows:

$$
\begin{aligned}
& 0=\text { no crystallization } \\
& 1=\text { atypical fern formation } \\
& 2=\text { primary and secondary stem ferning } \\
& 3=\text { tertiary and quaternary stem ferning }
\end{aligned}
$$

\section{A5.3.4 Spinnbarkeit}

Place a drop of cervical mucus on a microscope slide and touch it with a coverslip or a second slide held crosswise; then gently lift the coverslip or second slide. Estimate the length of the cervical mucus thread stretched between the two surfaces. 
Spinnbarkeit is scored as follows:

$$
\begin{aligned}
& 0=<1 \mathrm{~cm} \\
& 1=1-4 \mathrm{~cm} \\
& 2=5-8 \mathrm{~cm} \\
& 3=9 \mathrm{~cm} \text { or more }
\end{aligned}
$$

\section{A5.3.5 Cellularity}

It is recommended that all cell counts be expressed in cells per $\mu$ l. An estimate of the number of leukocytes and other cells in the cervical mucus is traditionally based on the number counted per high-power microscope field (HPF) (see Box A5.2).

\section{Box A5.2 Volume observed per high-power field in a $100-\mu \mathrm{m}$-deep mucus} preparation

The volume of mucus observed in each microscope field depends on the area of the field $\left(\pi r^{2}\right.$, where $\pi$ is approximately 3.142 and $r$ is the radius of the microscopic field) and the depth of the chamber (here $100 \mu \mathrm{m}$ ). The diameter of the microscope field can be measured with a stage micrometer or can be estimated by dividing the diameter of the aperture of the ocular lens by the magnification of the objective lens.

With a $\times 40$ objective and a $\times 10$ ocular of aperture $20 \mathrm{~mm}$, the microscope field has a diameter of approximately $500 \mu \mathrm{m}(20 \mathrm{~mm} / 40)$. In this case, $r=250 \mu \mathrm{m}, \mathrm{r}^{2}=$ $62500 \mu \mathrm{m}^{2}, \pi \mathrm{r}^{2}=196375 \mu \mathrm{m}^{2}$ and the volume is $19637500 \mu \mathrm{m}^{3}$ or about $20 \mathrm{nl}$.

Thus, a count of 10 cells per HPF is approximately equivalent to 10 cells per $20 \mathrm{nl}$, or 500 cells per $\mu \mathrm{l}$. As the number of cells counted is low, the sampling error is high; a replicate count of 10 has a sampling error of $22 \%$ (see Table 2.2 ), so the value could lie anywhere between 280 and 720 cells per $\mu$ l.

The rank scores for cells are:

$$
\begin{aligned}
& 0=>20 \text { cells per HPF or }>1000 \text { cells per } \mu \mathrm{l} \\
& 1=11-20 \text { cells per HPF or } 501-1000 \text { cells per } \mu \mathrm{l} \\
& 2=1-10 \text { cells per HPF or } 1-500 \text { cells per } \mu \mathrm{l} \\
& 3=0 \text { cells }
\end{aligned}
$$

A5.3.6 $\mathrm{pH}$

The $\mathrm{pH}$ of cervical mucus from the endocervical canal should be measured with $\mathrm{pH}$ paper, range 6.0-10.0, in situ or immediately following collection. If the $\mathrm{pH}$ is measured in situ, care should be taken to avoid touching the exocervical mucus, which always has a pH lower (more acidic) than that of mucus in the endocervical canal. Care should also be taken to avoid contamination with secretions of the vagina, which have a low $\mathrm{pH}$.

Spermatozoa are susceptible to changes in $\mathrm{pH}$ of the cervical mucus. Acid mucus immobilizes spermatozoa, whereas alkaline mucus may enhance motility. Exces- 
sive alkalinity of the cervical mucus ( $\mathrm{pH}$ greater than 8.5), however, may adversely affect the viability of spermatozoa. The optimum $\mathrm{pH}$ value for sperm migration and survival in the cervical mucus is between 7.0 and 8.5 , which is the normal pH range of mid-cycle cervical mucus. Although a $\mathrm{pH}$ value between 6.0 and 7.0 may be compatible with sperm penetration, motility is often impaired below $\mathrm{pH} 6.5$ and sperm-cervical mucus interaction tests are often not performed if the $\mathrm{pH}$ of mucus is below 7.0.

In some cases cervical mucus may be substantially more acidic. This can be due to abnormal secretions, the presence of a bacterial infection, or contamination with vaginal fluid.

\section{References}

Eggert-Kruse W et al. (1989). Prognostic value of in-vitro sperm penetration into hormonally standardized human cervical mucus. Fertility and Sterility, 51:317-323.

Eggert-Kruse W et al. (1993). The $\mathrm{pH}$ as an important determinant of sperm-mucus interaction. Fertility and Sterility, 59:617-628.

Insler V et al. (1972). The cervical score. A simple semiquantitative method for monitoring of the menstrual cycle. International J ournal of Gynaecology and Obstetrics, 10:223-228.

Moghissi KS (1976) Postcoital test: physiological basis, technique and interpretation. Fertility and Sterility, 27:117-129. 


\section{APPENDIX 6 Record forms for semen and cervical mucus analyses}

\section{A6.1 Template for a semen analysis recording form}

This sample record form overpage is offered as a model. It allows recording of observations made during semen analysis, using the methods described in this manual. It may be adapted to include derived variables, which are combinations of results from the primary data (e.g. total number of peroxidase-positive cells per ejaculate). When used for research purposes, data from the sample record form can be entered directly into a computer database, and any derived variables can be computed electronically.

The sample record form has multiple columns for recording the results of semen analyses performed at different times. This is a convenient way of presenting serial semen sample results. It may be useful to add extra space in certain parts of the form to allow the recording of additional comments and observations. Reference limits and consensus threshold values (see Appendix 1, Table 1.1 and comments), are given in square brackets, where available. 


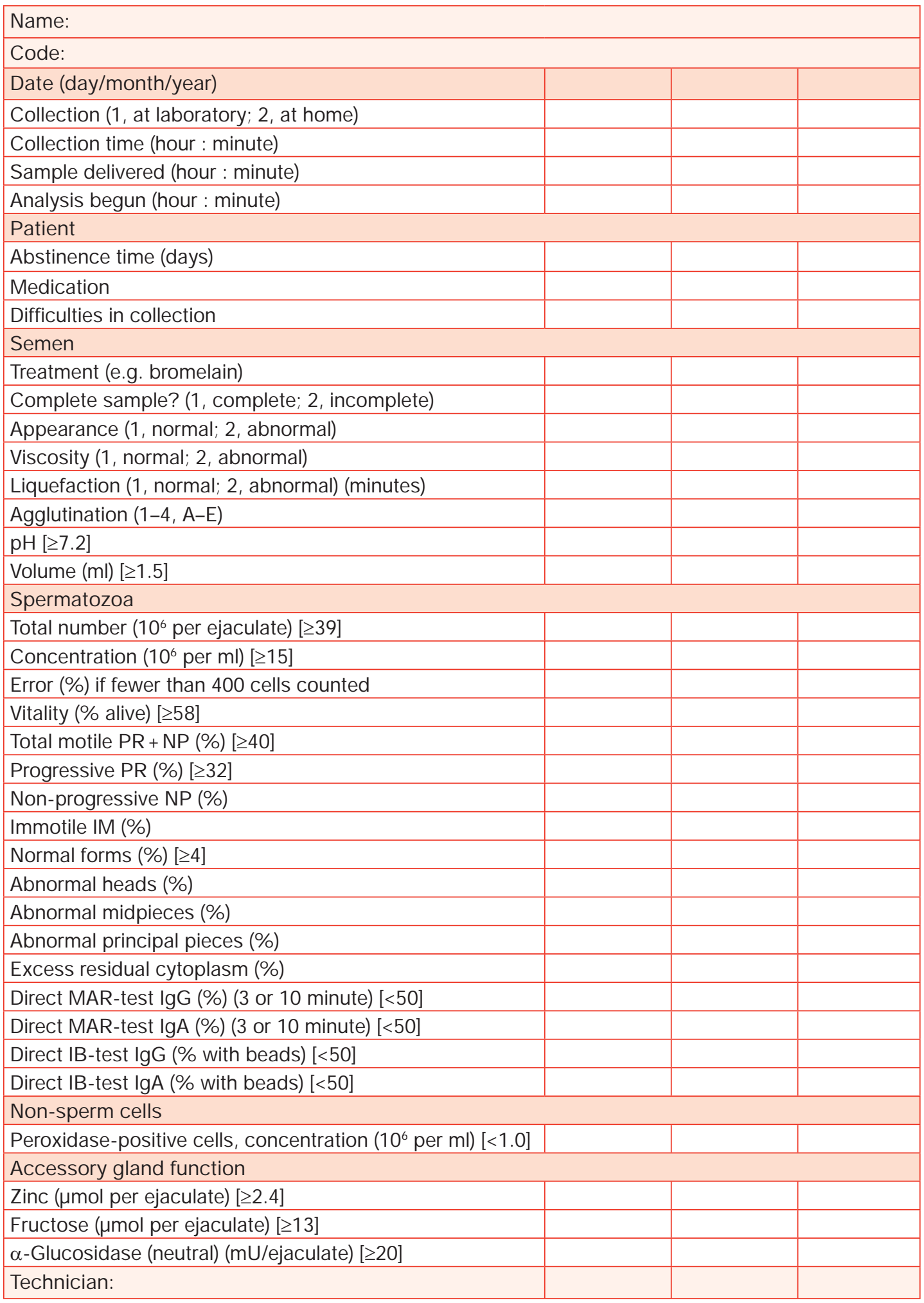




\section{A6.2 Template for a cervical mucus recording form}

\begin{tabular}{|l|}
\hline Name: \\
\hline Code: \\
\hline Date of first day of last menstrual period (day/month/year): \\
\hline
\end{tabular}

\begin{tabular}{|l|l|l|l|l|}
\hline \multicolumn{4}{|c|}{ Daily cervical mucus score } \\
\hline Date (day/month/year) & & & & \\
\hline Day of cycle & & & & \\
\hline Volume $(0,1,2,3)$ & & & & \\
\hline Viscosity $(0,1,2,3)$ & & & & \\
\hline Ferning $(0,1,2,3)$ & & & & \\
\hline Spinnbarkeit $(0,1,2,3)$ & & & & \\
\hline Cellularity $(0,1,2,3)$ & & & & \\
\hline Total score (max. 15$)$ & & & & \\
\hline pH & & & & \\
\hline
\end{tabular}

\begin{tabular}{|c|c|c|c|c|c|c|}
\hline \multicolumn{7}{|c|}{ Postcoital test } \\
\hline \multicolumn{7}{|l|}{ Date (day/month/year) } \\
\hline \multicolumn{7}{|l|}{ Time after coitus (hours) } \\
\hline & $\begin{array}{l}\text { Vaginal } \\
\text { pool }\end{array}$ & $\begin{array}{c}\text { Endocervical } \\
\text { pool }\end{array}$ & $\begin{array}{c}\text { Vaginal } \\
\text { pool }\end{array}$ & $\begin{array}{c}\text { Endocervical } \\
\text { pool }\end{array}$ & $\begin{array}{c}\text { Vaginal } \\
\text { pool }\end{array}$ & $\begin{array}{c}\text { Endocervical } \\
\text { pool }\end{array}$ \\
\hline \multicolumn{7}{|l|}{$\begin{array}{l}\text { Sperm concentration } \\
\text { (spermatozoa per } \mu \mathrm{l})\end{array}$} \\
\hline \multicolumn{7}{|l|}{ Sperm motility } \\
\hline \multicolumn{7}{|l|}{ PR $(\%)$} \\
\hline \multicolumn{7}{|l|}{ NP $(\%)$} \\
\hline \multicolumn{7}{|l|}{ IM $(\%)$} \\
\hline Technician: & & & & & & \\
\hline
\end{tabular}




\section{APPENDIX $7 \quad$ Sampling errors and quality control}

\section{A7.1 Errors in measurement of sperm concentration}

\section{A7.1.1 Errors in assessing counts}

To measure sperm concentration, the number of spermatozoa in a fixed volume of diluted semen is assessed in a counting chamber. However, a single estimate is of limited value without some indication of its precision. This is provided by the confidence interval, which has a specific probability (the confidence coefficient or coverage possibility) of containing the true value. The most commonly used probability is 0.95 . The interval is then called the $95 \%$ confidence interval, and the ends of this interval are the $95 \%$ confidence limits (Armitage et al., 2002).

If spermatozoa are randomly distributed throughout the chamber, the number in a given volume follows the Poisson distribution, with variance equal to the number counted. The standard error $(\mathrm{SE})$ of a count $(\mathrm{N})$ is its square root $(\sqrt{ } \mathrm{N})$, the sampling error $(\% \mathrm{SE})$ is $100 \times(\sqrt{\mathrm{N}} / \mathrm{N})$ and the $95 \%$ confidence interval $(\mathrm{CI})$ is approximately $\mathrm{N} \pm 1.96 \times \mathrm{SE}$ (or $\mathrm{N} \pm$ approximately $2 \times \mathrm{SE}$ ).

Note: These values are only approximate, as the confidence limits are not always symmetrical about the estimate. The exact $95 \%$ confidence interval, based on the properties of the Poisson distribution, is $361.76-441.21$ for a count of $400,81.36$ 121.66 for a count of $100,4.80-18.39$ for a count of $10,0.025-5.572$ for a count of 1 , and $0.0-3.7$ for a count of 0 .

\section{A7.1.2 Agreement between replicate counts}

Replicate counts on two separate dilutions of each semen sample are recommended, to account for possible uneven distribution of spermatozoa despite thorough mixing (see Section 2.4.1). Assessing the same chamber twice, or assessing both sides of one chamber filled from a single dilution, is not true replication, as this will not allow errors of preparation, mixing or dilution to be detected.

The difference between independent counts is expected to be zero, with standard error equal to the square root of the sum of the two counts. Thus $\mathrm{z}=$ (N1$\mathrm{N} 2) / \sqrt{ }(\mathrm{N} 1+\mathrm{N} 2)$ should be $<1.96$ by chance alone; if it is, the values are accepted. If $z$ is $>1.96$, new replicate dilutions are made. Fig. A7.1 gives the acceptable rounded values for N1-N2.

For example, for a mean count of 200 spermatozoa (sum 400), the difference between the replicate counts could be as large as 39, so the two counts could be $180.5(200-19.5)$ and $219.5(200+19.5)$ by chance alone.

Table A7.1 summarizes the data shown in Fig. A7.1 and can be used to assess the agreement between replicate counts (see Sections 2.8.3 and 2.11).

For routine sperm counting, it is recommended that at least 200 spermatozoa are counted in each replicate, so that a total of about 400 cells are counted; the sampling error is then less than $5 \%$ (see Table 2.2). With very low sperm numbers, higher sampling errors may be unavoidable (see Sections 2.11.1 and 2.11.2), in which case the sampling error (\% SE) for the number of spermatozoa counted (see Table 2.2) should be reported. 
Fig. A7.1 Acceptable differences between two replicate counts as a function of the total number of spermatozoa assessed

The line shows the maximum difference between replicate counts that is expected to occur by chance alone.

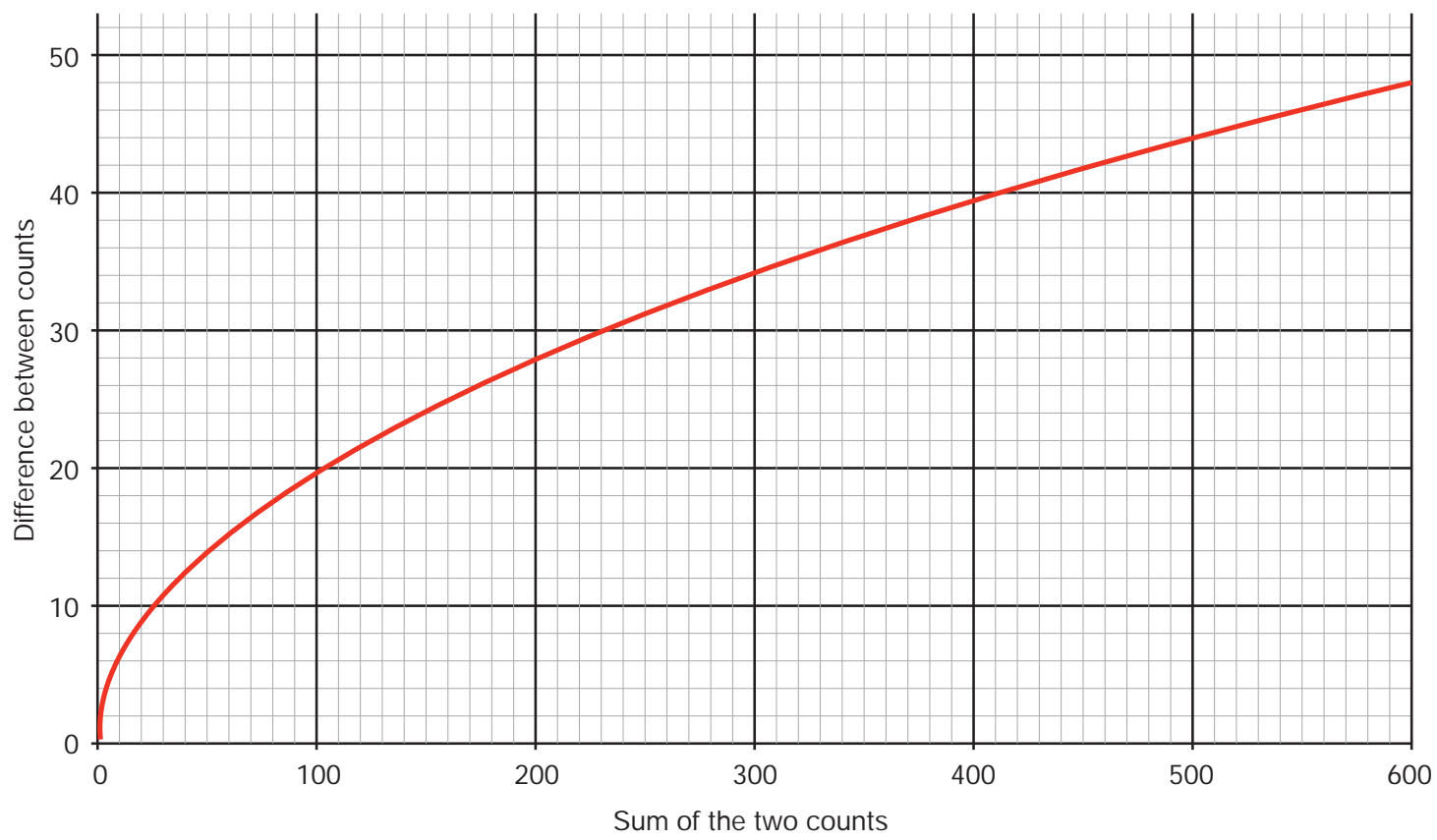

Table A7.1 Acceptable differences between two replicate counts for a given sum

\begin{tabular}{|c|c|}
\hline Sum & Difference* \\
\hline $35-40$ & 12 \\
\hline $41-47$ & 13 \\
\hline $48-54$ & 14 \\
\hline $55-62$ & 15 \\
\hline $63-70$ & 16 \\
\hline $71-79$ & 17 \\
\hline $80-89$ & 18 \\
\hline $90-98$ & 19 \\
\hline $99-109$ & 20 \\
\hline $110-120$ & 21 \\
\hline $121-131$ & 22 \\
\hline $132-143$ & 23 \\
\hline
\end{tabular}

\begin{tabular}{|c|c|}
\hline Sum & Difference* \\
\hline $144-156$ & 24 \\
\hline $157-169$ & 25 \\
\hline $170-182$ & 26 \\
\hline $183-196$ & 27 \\
\hline $197-211$ & 28 \\
\hline $212-226$ & 29 \\
\hline $227-242$ & 30 \\
\hline $243-258$ & 31 \\
\hline $259-274$ & 32 \\
\hline $275-292$ & 33 \\
\hline $293-309$ & 34 \\
\hline $310-328$ & 35 \\
\hline
\end{tabular}

\begin{tabular}{|c|c|}
\hline Sum & Difference* \\
\hline $329-346$ & 36 \\
\hline $347-366$ & 37 \\
\hline $367-385$ & 38 \\
\hline $386-406$ & 39 \\
\hline $407-426$ & 40 \\
\hline $427-448$ & 41 \\
\hline $449-470$ & 42 \\
\hline $471-492$ & 43 \\
\hline $493-515$ & 44 \\
\hline $516-538$ & 45 \\
\hline $539-562$ & 46 \\
\hline $563-587$ & 47 \\
\hline
\end{tabular}

*Based on rounded $95 \%$ confidence interval. 


\section{A7.2 The importance of understanding sampling errors}

This manual places great emphasis on counting a sufficient number of spermatozoa and getting replicate estimates to agree within certain limits. This is necessary because these procedures increase the certainty that the concentrations or total counts generated are close to the true (but unknown) values. If to o few spermatozoa are counted, the concentration calculated will be imprecise. If it is not possible to count a total of at least 400 spermatozoa, this should be stated on the report form and the error involved noted (see Table 2.2).

Precision is best achieved by counting in deep chambers, with large grid areas that contain large numbers of spermatozoa, rather than in shallow chambers with small grids containing few spermatozoa. To facilitate counting, the semen should be diluted sufficiently in fixative so that there is little overlap of non-motile cells. The example below illustrates the difference between chambers in achieving an accurate measurement for a semen sample with a low concentration of sperm.

For a low-volume chamber with a $1 \mathrm{~mm} \times 1 \mathrm{~mm}$ grid filled with undiluted spermatozoa:

- If the true sperm concentration is $1 \times 10^{6}$ per $\mathrm{ml}$ there are 1000 spermatozoa per $\mu$ l or 1 spermatozoon per $\mathrm{nl}$.

- In a $10-\mu \mathrm{m}$-deep chamber with a $1 \mathrm{~mm} \times 1 \mathrm{~mm}$ grid on the floor, there will be 10 spermatozoa in the entire $10 \mathrm{nl}$ grid.

- The error associated with counting only 10 spermatozoa is $32 \%$ and the $95 \%$ confidence interval $10 \pm 1.96 \times \sqrt{ } \mathrm{N}(=10 \pm 6.2)$ (see Table 2.2).

- This large confidence interval means that the true count could be between 4 spermatozoa $(10-6)$ and 16 spermatozoa $(10+6)$ in the total $10-n l$ volume.

- Thus, the estimate of the concentration is between 400000 and 1600000 spermatozoa per $\mathrm{ml}$ of semen.

- In practice, this means that the best estimate for a $50-\mu$ volume is that it contains between 20000 and 80000 spermatozoa.

- If two replicate preparations had been examined, the corresponding values for the 20 spermatozoa observed would be, from the $22 \%$ error, a confidence interval of $20 \pm 8.8$, actual numbers of $11(20-9)$ or $29(20+9)$ in the total $20 \mathrm{nl}$ volume, an estimate of the true value ranging from $550000 \mathrm{spermatozoa} / \mathrm{ml}$ to 1450000 spermatozoa/ml semen and between 27500 and 72500 spermatozoa per $50-\mu$ l aliquot.

For a large-volume chamber with nine $1 \mathrm{~mm} \times 1 \mathrm{~mm}$ grids filled with $1+1(1: 2)$ diluted semen:

- If the true sperm concentration is $1 \times 10^{6}$ per $\mathrm{ml}$ and a dilution of $1+1(1: 2)$ is made (see Section 2.8), there will be 500000 spermatozoa per $\mathrm{ml}, 500$ spermatozoa per $\mu$ l or 0.5 spermatozoa per $\mathrm{nl}$.

- In a 100- $\mu \mathrm{m}$-deep chamber with several $1 \mathrm{~mm} \times 1 \mathrm{~mm}$ grids on the floor $(100 \mathrm{nl}$ per grid) there will be 200 spermatozoa in four grids $(400 \mathrm{nl}), 400$ in the two replicates $(800 \mathrm{nl})$. 
- The error associated with counting 400 spermatozoa is $5 \%$ and the $95 \%$ confidence interval $400 \pm 1.96 \times \sqrt{ } \mathrm{N}(=400 \pm 39)$ (see Table 2.2).

- This confidence interval means that the true count could be between 360 spermatozoa $(400-40)$ and 440 spermatozoa $(400+40)$ in the total volume of $800 \mathrm{nl}$ of $1+1(1: 2)$ diluted semen.

- Thus, the estimate of the concentration is between 900000 and 1100000 spermatozoa per $\mathrm{ml}$ of undiluted semen.

- In practice, this means that a 50- $\mu$ l volume contains between 45000 and 55000 spermatozoa.

\section{A7.3 Errors in measurement of percentages}

\section{A7.3.1 Errors in assessing percentages}

When spermatozoa are classified into two classes (such as normal or abnormal morphology, motile or immotile, alive or dead, acrosome-reacted or not, fused with zona-free hamster eggs or not), the percentages follow the binomial distribution. For this distribution, the standard error of the estimated percentage $(p)$ within a class depends on the true, but unknown, percentage, as well as on the number of spermatozoa counted $(N)$. The standard error is $\sqrt{ }(p(100-p) / N)$, and an approximate confidence interval can be constructed from the normal distribution. This is a good approximation for values in the range $20-80 \%$.

- If 100 spermatozoa are counted, and the percentage with normal morphology is $20 \%$, the standard error of the estimated percentage of normal spermatozoa is $\sqrt{ }(20(100-20) / 100)=\sqrt{ }((20 \times 80) / 100)=\sqrt{ }(1600 / 100)=4 \%$. The $95 \%$ confidence limit is $\pm 1.96 \times 4 \%$ or $\pm 7.8 \%$, and the corresponding confidence interval $12.2-27.8 \%$.

- If 200 spermatozoa are counted, the standard error is $\sqrt{ }(20(100-20) / 200)$ $=\sqrt{ }((20 \times 80) / 200)=\sqrt{ }(1600 / 200)=2.8 \%$. The $95 \%$ confidence limit is $\pm 1.96 \times 2.8 \%$ or $\pm 5.5 \%$, and the corresponding confidence interval $14.5-25.5 \%$.

- If 400 spermatozoa are counted, the standard error is $\sqrt{ }(20(100-20) / 400)=$ $\sqrt{ }((20 \times 80) / 400)=\sqrt{ }(1600 / 400)=2.0 \%$. The $95 \%$ confidence limit is $\pm 1.96 \times 2 \%$ or $\pm 3.9 \%$ and the corresponding confidence interval $16.1-23.9 \%$.

Outside the range $20-80 \%$, it is more appropriate to use the angular transformation (arc sin square root) $z=\sin ^{-1} \sqrt{ }(p / 100)$. This has the property that the standard deviation of $z$ is $1 /(2 \sqrt{ } \mathrm{N})$ and thus depends only on the number of spermatozoa counted and not the true (but unknown) percentage. An alternative is to compute exact binomial confidence limits using one of several widely available statistical software packages.

\section{A7.3.2 Agreement between replicate percentages}

It is recommended that replicate assessments of percentages $\left(p_{1}\right.$ and $\left.p_{2}\right)$ are made on $\mathrm{N}$ spermatozoa in each sample and compared. The limit of expected dif- 
ference $d$ (where $\left.d=\left|p_{1}-p_{2}\right|\right)$ is $1.96\left(\sqrt{ }(2 \bar{p}(100-\bar{p}) / N)\right.$ where $\bar{p}=\left(p_{1}+p_{2}\right) / 2$. The difference between independent assessments is expected to be zero, with standard error dependent on the estimated percentage and the total number of spermatozoa counted.

The large statistical errors associated with counting fewer than 200 spermatozoa per replicate are apparent in Fig. A7.2, which shows the exact $95 \%$ confidence intervals for agreement between percentages for replicate counts of 100, 200 and 400 spermatozoa (i.e. total sperm numbers of 200, 400 and 800 ). It also shows that the error is symmetrical around $50 \%$, with a maximum at $50 \%$ and minima at $0 \%$ and $100 \%$.

Fig. A7.2 The acceptable differences between two replicate assessments of percentage as a function of the true percentage and the total number of spermatozoa assessed

The lines show the differences that are expected to occur by chance alone (95\% confidence limits) for replicate estimated percentages from 100 (total 200: top, solid line), 200 (total 400: middle, dotted line) and 400 (total 800: lower, dashed line) spermatozoa.

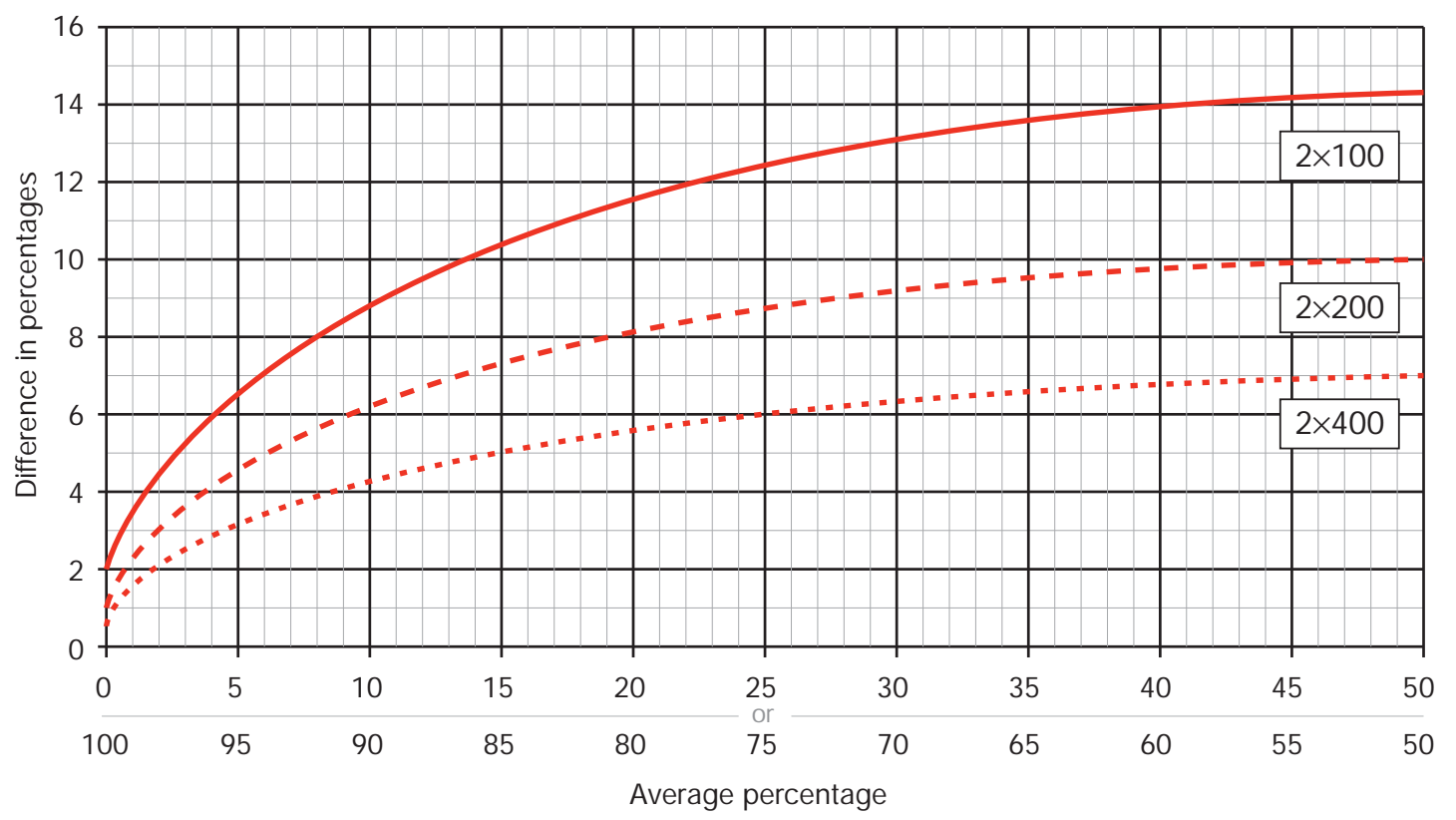

The acceptable differences between replicates can be read from this graph. For a total of 200 spermatozoa (100 per replicate) and a true percentage of $5 \%$ (or $95 \%$ ), the upper $95 \%$ confidence limit for the difference is $6.6 \%$. On average, 19 of 20 repeated assessments of the same sample will be between $2.42 \%$ and $9.00 \%$; one in 20 will give a result outside these limits by chance alone. For a total of 800 spermatozoa (400 per replicate: dashed line) and a true percentage of $5 \%$ (or $95 \%$ ), the upper $95 \%$ confidence limit for the difference is $3.1 \%$, and the $95 \%$ confidence limits are $3.1 \%$ and $7.6 \%$. Similarly, if a total of 400 spermatozoa is counted (200 per replicate; dotted line), for a true value of $20 \%$ (or $80 \%$ ) the upper $95 \%$ confidence limit is $8.1 \%$, with limits $16.2 \%$ and $24.3 \%$. 
Tables A7.2, A7.3 and A7.4 present data on the acceptable differences between replic ates (those occurring by chance alone) for a range of percentages estimated from different numbers of total sperm counted. These may be more useful than the graph (Fig. A7.2) for assessing the agreement between replicate percentages of spermatozoa that are morphologically normal, motile, viable or acrosome-reacted.

Table A7.2 Acceptable differences between two percentages for a given average, determined from replicate counts of 100 spermatozoa (total 200 counted)

\begin{tabular}{|c|c|}
\hline Average (\%) & Difference* \\
\hline 0 & 2 \\
\hline 1 & 3 \\
\hline 2 & 4 \\
\hline 3 & 5 \\
\hline 4 & 6 \\
\hline $5-6$ & 7 \\
\hline $7-9$ & 8 \\
\hline $10-12$ & 9 \\
\hline $13-15$ & 10 \\
\hline $16-19$ & 11 \\
\hline $20-25$ & 12 \\
\hline $26-33$ & 13 \\
\hline $34-66$ & 14 \\
\hline
\end{tabular}

\begin{tabular}{|c|c|}
\hline Average (\%) & Difference* \\
\hline $67-74$ & 13 \\
\hline $75-80$ & 12 \\
\hline $81-84$ & 11 \\
\hline $85-87$ & 10 \\
\hline $88-90$ & 9 \\
\hline $91-93$ & 8 \\
\hline $94-95$ & 7 \\
\hline 96 & 6 \\
\hline 97 & 5 \\
\hline 98 & 4 \\
\hline 99 & 3 \\
\hline 100 & 2 \\
\hline
\end{tabular}

*Based on rounded $95 \%$ confidence interval.

Table A7.3 Acceptable differences between two percentages for a given average, determined from replicate counts of 200 spermatozoa (total 400 counted)

\begin{tabular}{|c|c|}
\hline Average $(\%)$ & Difference* $^{*}$ \\
\hline 0 & 1 \\
\hline 1 & 2 \\
\hline 2 & 3 \\
\hline $3-4$ & 4 \\
\hline $5-7$ & 5 \\
\hline $8-11$ & 6 \\
\hline $12-16$ & 7 \\
\hline $17-23$ & 8 \\
\hline $24-34$ & 9 \\
\hline $35-65$ & 10 \\
\hline
\end{tabular}

\begin{tabular}{|c|c|}
\hline Average $(\%)$ & Difference* $^{*}$ \\
\hline $66-76$ & 9 \\
\hline $77-83$ & 8 \\
\hline $84-88$ & 7 \\
\hline $89-92$ & 6 \\
\hline $93-95$ & 5 \\
\hline $96-97$ & 4 \\
\hline 98 & 3 \\
\hline 99 & 2 \\
\hline 100 & 1 \\
\hline
\end{tabular}

*Based on rounded $95 \%$ confidence interval. 
Table A7.4 Acceptable differences between two percentages for a given average, determined from replicate counts of 400 spermatozoa (total 800 counted)

\begin{tabular}{|c|c|}
\hline Average $(\%)$ & Difference* $^{*}$ \\
\hline 0 & 0 \\
\hline $1-3$ & 2 \\
\hline $4-6$ & 3 \\
\hline $7-11$ & 4 \\
\hline $12-18$ & 5 \\
\hline $19-30$ & 6 \\
\hline $31-69$ & 7 \\
\hline
\end{tabular}

\begin{tabular}{|c|c|}
\hline Average (\%) & Difference* $^{*}$ \\
\hline $70-81$ & 6 \\
\hline $82-88$ & 5 \\
\hline $89-93$ & 4 \\
\hline $94-96$ & 3 \\
\hline $97-99$ & 2 \\
\hline 100 & 0 \\
\hline
\end{tabular}

*Based on rounded $95 \%$ confidence interval.

\section{A7.4 Production of semen samples for quality control}

Quality control specimens should ideally be representative of the range of semen samples processed in the laboratory. If only a small number of $\mathrm{QC}$ samples are to be analysed, they should be those most relevant to the main activity in the laboratory. For example, in the laboratory of an infertility service, clinically significant ranges (concentration $15 \times 10^{6}$ to $50 \times 10^{6}$ per ml, progressive motility $30-50 \%$, and normal morphology below $5 \%$ ) could be chosen.

- Aliquots of pooled semen samples can be stored frozen, or at $4{ }^{\circ} \mathrm{C}$ with a preservative, and analysed at intervals for sperm concentration.

- Spermatozoa may not survive cryopreservation sufficiently well to be a useful source of internal and external QC materials for motility and sperm antibody tests.

- Video tapes, CDs and DVDs can also be used for sperm motility.

- Photographs, video tapes, CDs and DVDs can be used for sperm morphology.

- Video-recordings are particularly useful for training in motility and morphology assessment, but their use should complement, not replace, replicate assessments of semen specimens.

- Stained semen slides can be used for morphology quality control. Fixed smears can also be stored and used to monitor staining. Stained slides may deteriorate with time, depending on the quality of the fixing or staining procedure. However, slides stained using the Papanicolaou procedure described in this manual, and stored in the dark at room temperature, should last for months or even years.

- Sperm antibody-positive serum may be used for QC of indirect immunobead tests, but is not recommended for use in direct immunobead tests. 


\section{A7.5 Preparation of a video-recording for internal quality control of analysis of sperm motility}

This protocol describes how to prepare a video-recording to be used for quality control of manual motility assessment procedures.

- Record at least five and up to 10 fields to mimic the multiple fields assessed for motility analysis during semen evaluation and to allow at least 400 spermatozoa to be assessed.

- The video-recording should contain images from several different semen samples, covering the range of motilities typically seen during routine semen evaluation.

- The videotape can simply have five fields of a few different semen specimens; in other cases, a more complex recording may be needed, for example for standardization between laboratories or in a multicentre study. In this case, more semen samples might be used, and the samples could be repeated randomly throughout the videotape. Repeated samples allow intra-technician precision to be estimated.

A7.5.1 Additional equipment

In addition to the routine equipment for estimating motility, the preparation of recordings for quality control requires:

- a video-recorder or computer with a CD-RW or DVD-RW drive;

- a marking device for coding the video-recording, such as a slide with numbers etched on its surface (an England finder) or time generator.

A7.5.2 Procedure

- If several semen samples are available, the entire video-recording can be prepared at one session; otherwise, samples can be recorded as they become available.

- If motility is typically assessed at room temperature, the recordings should be done at room temperature. Likewise, if motility is typically assessed at $37^{\circ} \mathrm{C}$, then the recordings should be made at the same temperature.

Note: If recording is to be done at $37^{\circ} \mathrm{C}$, the stage warmer should be turned on and allowed to reach a stable temperature at least 10 minutes before use.

- Prepare a recording of sufficient fields to ensure that 400 spermatozoa are recorded from several different semen samples.

- For specimens with low semen concentration, more than 10 fields may be necessary to give adequate numbers of spermatozoa for scoring. Video-recording of 10 fields will take several minutes.

- The video-recording can be done when either a slide with coverslip or a fixed 20- $\mu \mathrm{m}$-deep chamber is used for the analysis. 
Note 1: When disposable counting chamber slides are used, motility will be stable for a longer period of time than when slides and coverslips are used. This will allow 10 (or more) fields to be recorded from the same preparation.

Note 2: When slides and coverslips are used, it may be necessary to use several during the video-recording to avoid a noticeable decline in motility over time.

- Identify several semen samples with a range of motility values.

- Each specimen should have a unique code on the video-recording. The coding can vary from simply marking each specimen, to marking each field of each specimen. For example, the first specimen marker could be at the beginning of the first field, with no other coding until the second specimen appears. Alternatively, the coding could include markings of each individual field, i.e. the first field of the first specimen would be marked 01-01, the second field of the first specimen would be marked 01-02, etc. This more elaborate marking system helps the technicians track where they are during analysis.

Note 1: It is useful to have short blank sections on the video-recording between fields or between specimens. This allows the technician to recognize the beginning of the new segment.

Note 2: The easiest way to get a blank segment when recording is to cover the light source.

Note 3: This can also be done before pausing the video-recorder; the "pause" should always be used rather than the "stop" button, as the "stop" button may cause noise or static on the videotape.

- Record an image of a stage micrometer for 10 seconds at the magnification that will be used for recording the samples. The magnification should provide an image on the monitor similar to that used for visual microscopic analysis. The stage micrometer image gives a permanent record of the magnification, which permits calibration of the screen-overlapping acetate grid for use during analysis of the videotape or calibration of a CASA instrument.

- Record the coding image for the first specimen for 5-7 seconds. At the end of this time, block the light source for 3 seconds to give a blank image to serve as a marker; then pause the recording.

- Identify the first semen specimen to be used for recording. Place $10 \mu$ l of wellmixed semen on a glass slide and cover with a $22 \mathrm{~mm} \times 22 \mathrm{~mm}$ coverslip, or load a fixed slide chamber with $7 \mu$ l of well-mixed semen. Allow the sample to settle for a few seconds (at $37{ }^{\circ} \mathrm{C}$ if required) until drifting has stopped. Record 10 (or more) fields, following the pattern shown in Fig. A7.3. For CASA QC, the sperm concentration should not exceed $50 \times 10^{6}$ per ml; more concentrated samples may have to be diluted in homologous seminal plasma (see Section 3.5.2). 
- Choose the first field near the upper left section of the coverslip or chamber, at least $5 \mathrm{~mm}$ from the edge. Record the field for 15 seconds, keeping the microscope and the stage as still as possible. After 15 seconds, record a 3-second blank and pause the recording. If individual fields are being coded, change the code number and record an image containing only the code number for 5-7 seconds.

- Following the pattern shown in Fig. A7.3, locate a second motile field on the slide or chamber, and record this field for 15 seconds. Again, record a 3-second blank at the end of the 15 seconds. Pause the recording and, if desired, change the code number to indicate the third field. Continue recording in this way until a total of at least 400 spermatozoa (10 fields or more, depending on the concentration) have been captured. After recording the final field and a 3-second blank, stop the recording.

- Prepare a second sample. Record the coding image for specimen two for 5-7 seconds, followed by a 3-second blank.

- Record the second sample according to the steps above, recording 10 or more fields for 15 seconds each, with a blank in between each field and a blank at the end of the final field.

- Repeat this process until the desired number of specimens have been video-recorded.

Fig. A7.3 Aid to assessing sperm motility

Systematic scanning of fields for video-recording of sperm motility at least $5 \mathrm{~mm}$ from the edges of the coverslip.

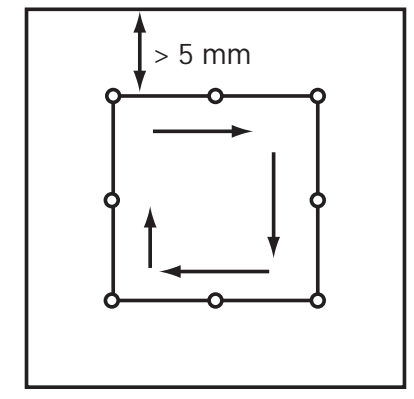

Note: If a more complex IQC motility video-recording, containing randomly repeated specimens, is desired, either a second recorder or a computer equipped with specialized video-editing software is required. In this case, each specimen should be video-recorded separately, with only the fields marked. The specimen number should not be recorded, as this will change as the specimen is repeated on the recording. If a computer equipped with video-editing software is available, images from each specimen can be digitized and combined as desired on a DVD. 


\section{A7.5.3 Analysis of the video-recording}

- Draw an acetate grid overlay and place it over the video monitor to be used during analysis of the video-recording, as detailed below. This will mimic the grid used in the eyepiece during microscopic analysis (see Fig. A7.4a).

Fig. A7.4 View through an ocular with reticle (red grid)

(a) ocular alone

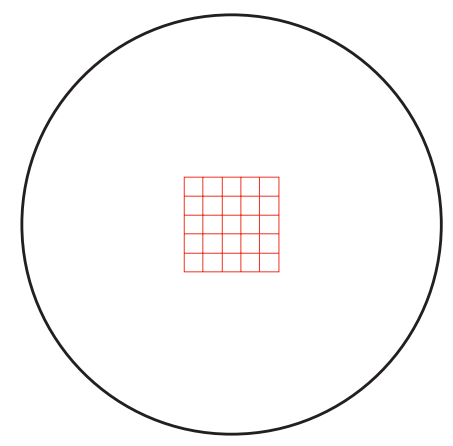

(b) view of stage micrometer

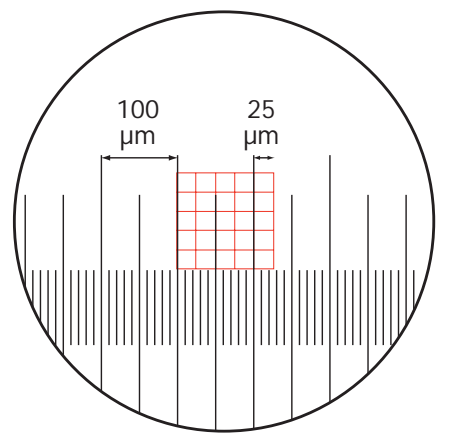

- Place the stage micrometer on the microscope stage at the magnification used for motility analysis. Looking through the ocular with reticle (see Fig. A7.4), measure the size of the grid sections using the stage micrometer. In this example the reticle grid is $125 \mu \mathrm{m} \times 125 \mu \mathrm{m}$ and each square is $25 \mu \mathrm{m} \times 25 \mu \mathrm{m}$ (Fig. A7.4b). Make a note of these measurements.

- Play the recording through the video monitor and pause at the image of the micrometer (Fig. A7.5a).

- Tape an acetate sheet over the screen and draw a square the size of one square in the eyepiece reticle grid, as measured above (see Fig. A7.5b).

- Complete the image of the entire eyepiece reticle grid (25 squares) (Fig. A7.5c).

- To analyse the video-recording, secure the acetate grid overlay over the video monitor. The analysis should be done on a standardized section of the grid overlay, e.g. the top two rows or the middle three rows.

- Score replicate assessments of 200 spermatozoa for each recorded segment.

Fig. A7.5 View of the videotaped image of the stage micrometer on the monitor and the drawn overlay; see text for explanation

(a)

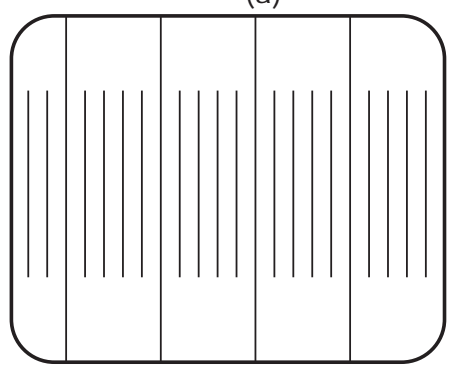

(b)

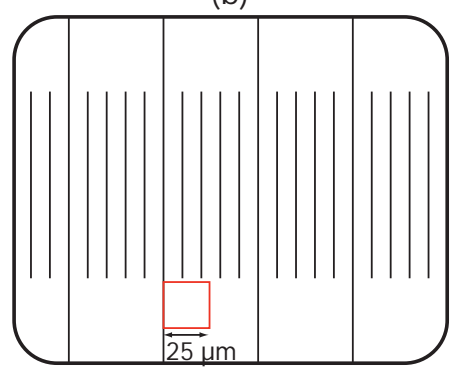

(c)

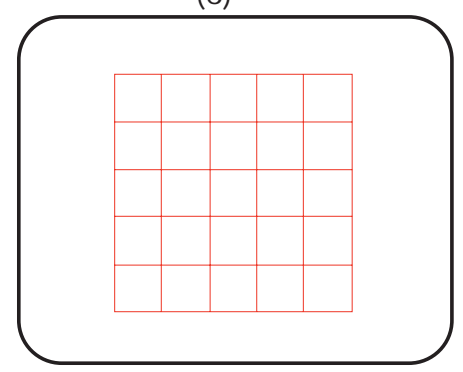




\section{A7.6 Preparation of diluted semen for internal quality control of determination of sperm concentration}

\section{A7.6.1 General considerations}

- Some steps of the procedure for determining sperm concentration in semen can be monitored using diluted, preserved semen samples prepared in the laboratory.

- The IQC samples should be representative of the range of concentrations normally seen in the laboratory during routine semen evaluation.

- Dilute the semen in a preservative, and place aliquots in storage vials. These can be refrigerated and used later for counting.

- Take care when preparing the suspensions to mix the specimen thoroughly, to ensure that vials prepared from the same specimen contain identical concentrations of spermatozoa. In this way, differences in counts on the IQC samples can be attributed to problems in the counting procedure.

- Dilute the preserved IQC samples again before assessing the concentration using a haemocytometer. Use the final dilution that is used in the laboratory during routine counting. This ensures that the concentration of background debris and other non-sperm cells will be similar to that seen during routine evaluation. For example, if the semen is initially diluted with an equal volume of preservative, an additional $1+9$ (1:10) dilution would yield a final dilution of $1: 20$.

- When a preserved sample with low sperm concentration is desired, it is better to start with a low concentration semen specimen rather than making a large dilution of a more concentrated specimen. This will ensure that the background is similar to that observed during routine semen analysis.

- Swim-up sperm preparations lack the debris, loose heads and cell fragment contamination seen during routine semen evaluation, and are best used only for monitoring the counting of similarly selected sperm suspensions.

- The number of sperm suspensions for IQC prepared at one time will depend on the number of technicians and the frequency of counting.

- Preserved diluted semen kept under refrigeration should be stable for at least 4 months.

\section{A7.6.2 Reagents}

Any of three preservatives may be used:

- Formalin: $10 \%$ (v/v) formaldehyde. To $27 \mathrm{ml}$ of purified water add $10 \mathrm{ml}$ of $37 \%$ (v/v) formaldehyde.

- Azide (J ørgensen et al., 2001): $3 \mathrm{~mol} / \mathrm{l}$ sodium azide $\left(\mathrm{NaN}_{3}\right)$. Dissolve $19.5 \mathrm{~g}$ of $\mathrm{NaN}_{3}$ in $100 \mathrm{ml}$ of purified water.

- Agglutination-preventing solution (APSIS) (B razil et al., 2004). To $100 \mathrm{ml}$ of purified water add $1.0 \mathrm{~g}$ bovine serum albumin (BSA), $2.0 \mathrm{~g}$ of polyvinylpyrrolidone (PVP), $0.90 \mathrm{~g}$ of sodium chloride ( $\mathrm{NaCl}), 0.1 \mathrm{ml}$ of detergent Triton $\mathrm{X}-100$, 
$0.004 \mathrm{ml}$ of silicone antifoaming agent and $0.10 \mathrm{~g}$ of sodium azide. Mix thoroughly and pass through a $0.45-\mu \mathrm{m}$ filter to eliminate debris. Store at $4{ }^{\circ} \mathrm{C}$.

Note: The bactericide sodium azide can be omitted from APSIS to make the solution non-toxic. However, such solutions should be discarded if contaminated.

\section{A7.6.3 Additional supplies}

In addition to the routine equipment for estimating sperm concentration, the preparation of QC samples requires:

- cryovials or other small tubes with tight-fitting lids for storage;

- permanent markers for labelling tubes.

\section{A7.6.4 Procedure}

1. Identify semen samples of the approximate desired concentration. The volume of preserved semen required will vary according to the needs of the laboratory; either use the entire volume of semen available or prepare $4 \mathrm{ml}$ of diluted sperm suspension for each concentration.

2. As soon as possible after collecting the semen, dilute it with preservative. If APSIS is used for dilution and preservation, the longer the time before dilution, the greater the chance of crystal formation following dilution. These crystals can interfere with loading the chamber and counting sperm.

3. Transfer the volume of semen required to a $15-\mathrm{ml}$ centrifuge tube. For each $\mathrm{ml}$ of semen, add either $100 \mu \mathrm{l}$ of $10 \%(\mathrm{v} / \mathrm{v})$ formalin, $10 \mu \mathrm{l}$ of $3 \mathrm{~mol} / \mathrm{l}$ azide, or $1 \mathrm{ml}$ of APSIS.

4. Label all vials to be used for storage of the samples with identifying information and the date of preparation. Lids or tops should be removed and the vials placed in a rack to permit quick and easy filling.

5. Make sure that the diluted, preserved semen is thoroughly mixed throughout the allocation process, to ensure that all vials contain similar sperm concentrations. Even minor delays after mixing can allow the spermatozoa to begin to settle, altering the concentration in the aliquots. One way to ensure constant mixing is to place the centrifuge tube of diluted semen in a rack, and then mix the semen continuously with one hand using a plastic transfer pipette, while removing the aliquots using a pipette in the other hand.

6. Depending on the needs of the laboratory, each vial should contain $0.5-1.0 \mathrm{ml}$. Storing the samples in $0.5-\mathrm{ml}$ aliquots allows several counts to be made from each vial.

7. Once the preserved sperm suspension has been distributed to all the vials, they should be tightly capped. Depending on the type of vial used, the lid can be sealed with a strip of self-sealing laboratory film. This is not necessary if cryovials are used.

8. Repeat the entire process for the remaining semen samples.

9. Store the vials at $4{ }^{\circ} \mathrm{C}$. 
Note: The concentration of the IQC solutions should be determined after the dilutions have been prepared, and should not be assumed from the original semen concentration. Once the preserved sperm suspensions have been prepared, a vial can be removed as needed and assessed (see Sections 2.7 and 2.8). The results can be charted using the procedure described in Section 7.7. All counts should be done using the counting method typically used in the laboratory. The section below describes the procedure using the haemocytometer.

\section{A7.6.5 Using the stored IQC samples}

- The preserved solutions must be further diluted before counting; the dilution will depend on the preservative used.

- The initial dilution of semen with formalin and azide is minimal, so does not need be taken into account. Semen preserved in APSIS is initially diluted twofold (i.e. $1+1(1: 2)$ ) and this must be taken into account in the final calculation of concentration.

- For suspensions diluted in APSIS from semen with an original concentration above $25 \times 10^{6}$ per $\mathrm{ml}$, counting is best accomplished using a further $1+9(1: 10)$ dilution. This can be obtained by adding $50 \mu$ of preserved sperm suspension to $450 \mu$ of purified water. This yields a final semen dilution of 1:20. Do not use APSIS as diluent, because this will interfere with the sperm settling on the haemocytometer grid.

- For the following steps, all pipettes should be preset to the appropriate volume and preloaded with a clean tip for quick removal of the aliquot immediately after mixing.

- A dilution vial should be prepared with the appropriate volume of water (i.e. $450 \mu$ l if making a 1:10 dilution as suggested above). The contents of the semen storage vial should be well mixed on a vortex mixer for approximately 30 seconds at maximum speed. A $50-\mu \mathrm{l}$ aliquot should then be transferred to the dilution vial containing water. The dilution vial should then be vortexed for 20 seconds at maximum speed. The haemocytometer should be loaded with $10 \mu$ l of suspension, and the spermatozoa counted as described in Sections 2.8.2 and 2.8.3.

- If the original semen sample used to prepare the preserved semen had a low concentration of spermatozoa, the dilution for counting will need to be adjusted accordingly. For example, if the original semen concentration was in the range of $4-25 \times 10^{6}$ per $\mathrm{ml}$, to create a final dilution of $1: 5$ as in the laboratory, the appropriate additional dilution of APSIS-preserved semen would be $2: 5(2+3$ : since the semen has already been diluted $1+1$ (1:2) with APSIS). This can be achieved by diluting $50 \mu$ l of the preserved semen with $75 \mu$ l of purified water.

- Preserved sperm suspensions stored in the refrigerator should be stable for at least 4 months, at which time new solutions should be prepared. It is desirable to have a period of overlap, during which the old and new preparations are both run, to monitor the transition period. 


\section{A7.7 Preparation of slides for internal quality control of assessment of sperm morphology}

\section{A7.7.1 General considerations}

- Smears can be prepared in the laboratory for use in internal quality control of morphology staining and analysis.

- Multiple smears can be prepared from each of several different semen samples, representing the range of morphology scored in the laboratory.

- The smears can be fixed and stored for later use in monitoring the staining and analysis procedures.

- Stained smears can be used individually or in replicate for QC of the morphology analysis procedure.

- Use of replicates allows intra-technician precision to be determined. These QC slides are also useful when comparing results from different technicians within a laboratory, or when comparing analyses between laboratories.

- Papanicolaou-stained and mounted smears, stored in the dark at room temperature, should be stable for many months or even years.

- The semen must be mixed thoroughly throughout the entire process of smear preparation, to ensure that all the smears prepared from a particular semen sample are identical. Any major variation detected during analysis can be presumed to be a result of the process being monitored (i.e. the morphology analysis procedure) and not caused by inadequate mixing of the semen during slide preparation.

\section{A7.7.2 Procedure}

1. Transfer the semen from the specimen container into a $15-\mathrm{ml}$ centrifuge tube. This will allow easier and more thorough mixing during the slide preparation process.

2. Clean both surfaces of frosted glass slides by rubbing vigorously with lint-free paper tissues.

3. Label the frosted slides with identifying information (e.g. identification number and date) using an HB (number 2 ) lead pencil. Pencil markings are stable through fixation and Papanicolaou staining of slides; ink markings from pens and some permanent markers are not.

4. Attach a clean tip to the pipette and set the volume to $10 \mu \mathrm{l}$ (or the volume routinely used in the laboratory for preparation of morphology smears).

5. The semen must be thoroughly mixed during the entire process, to ensure that all smears are as similar as possible. After mixing, even minor delays before removing the aliquot can allow the sperm to begin to settle, altering the population of spermatozoa delivered to the slide.

6. Mix the sample well in the centrifuge tube by aspirating it 10 times into a widebore (approximately $1.5 \mathrm{~mm}$ diameter) pipette equilibrated to the temperature of 
the sample. This process should be vigorous enough to mix the semen, yet not so vigorous that it creates bubbles.

7. Immediately after mixing, without allowing time for the spermatozoa to settle out of suspension, place $10 \mu \mathrm{l}$ of semen on the clear end of one of the cleaned slides. It is important not to let the drop of semen remain on the slide for more than a couple of seconds before smearing.

8. Smear the aliquot of semen over the surface of the slide using the feathering technique (see Section 2.13.2). In this procedure, the edge of a second slide is used to drag the drop of semen along the surface of the slide. Be sure to use the slide to "pull" the semen across the slide: do not use the slide to "push" the semen from behind. Care must be taken not to make the smears too thick, or there will be overlapping or clumped spermatozoa and more background stain. The separation of the spermatozoa on the slide depends on the volume of semen and the sperm concentration, the angle of the dragging slide (the smaller the angle, the thinner the smear) (Hotchkiss, 1945) and the speed of smearing (the more rapid the movement, the thicker the smear) (Eliasson, 1971).

9. Repeat steps 6-8 for the remaining slides, making only one slide after each mixing to ensure the spermatozoa do not settle before the aliquot is removed. If there is a pause of more than a couple of seconds after mixing, the semen should be remixed before the aliquot is removed.

10. Once the technique is established and the preparation is going smoothly, it may be possible to make two or three slides after each mixing. The aliquots should all be removed immediately after mixing, and the two or three smears made as quickly as possible, within a few seconds.

\section{A7.8 Calibration of equipment}

- Pipettes, counting chambers and other equipment should be calibrated at 6-monthly or yearly intervals.

\section{A7.8.1 Balances}

- Balances should be checked regularly with internal calibrators, and by external calibration at the time of regular laboratory maintenance service.

- Calibrate balances by weighing external standard weights (e.g. 1, 2, 5 and $10 \mathrm{~g}$ to cover the range of semen weights).

- Repeat measurements 10 times and calculate the mean, SD and coefficient of variation (CV) ( $=100 \times \mathrm{SD} /$ mean).

- Check the accuracy (that the stipulated weight falls within 2 SD of the measured mean).

\section{A7.8.2 Pipettes}

- Calibrate pipettes by aspirating purified water up to the graduation mark and dispensing into tared weighing boats.

- Calculate the anticipated volume from the weight of water pipetted assuming a density of $1 \mathrm{~g} / \mathrm{ml}$. 
Note: The density of water decreases with temperature (Lentner, 1981). It is $0.9982 \mathrm{~g} / \mathrm{ml}$ at $20{ }^{\circ} \mathrm{C}, 0.9956 \mathrm{~g} / \mathrm{ml}$ at $30{ }^{\circ} \mathrm{C}$ and $0.9922 \mathrm{~g} / \mathrm{ml}$ at $40{ }^{\circ} \mathrm{C}$. For purposes of calibration, however, an assumed value of $1.0 \mathrm{~g} / \mathrm{ml}$ is adequate.

- Repeat measurements 10 times and calculate the mean, SD and CV ( $=100 \times \mathrm{SD} /$ mean).

- Check the accuracy (that the stipulated volume falls within 2 SD of the measured mean).

\section{A7.8.3 Depths of chambers}

- Measure the depth of counting chambers using the Vernier scale on the fine focus of a microscope. Focus first on the chamber grid and then on an ink mark on the underside of the coverslip. Measure the number of graduation marks between the two points.

- Repeat the measurement 10 times and calculate the mean, SD and CV (= $100 \times \mathrm{SD} /$ mean).

- Check the accuracy (that the stipulated depth falls within 2 SD of the measured mean).

A7.8.4 Incubators

- The temperature of incubators and warm stages should be checked with thermometers that are, in turn, regularly calibrated.

- $\mathrm{CO}_{2}$ gas mixtures should be checked daily with the incubator readout, or by other gas analyser systems, weekly to monthly, and by gas sampling at the time of servicing.

\section{A7.8.5 pH paper}

- This should be checked against known pH standards.

A7.8.6 Other equipment

- Other laboratory equipment and reagents, such as pH meters, should also be checked against standards at 3- to 6-month intervals.

\section{References}

Armitage P et al. (2002). Statistical methods in medical research. Oxford, B lackwell Science.

B razil C et al. (2004). Quality control of laboratory methods for semen evaluation in a multicenter research study. J ournal of Andrology, 25:645-656.

Eliasson R (1971). Standards for investigation of human semen. Andrologia, 3:49-64.

Hotchkiss RS (1945). Fertility in man. London, William Heineman Medical Books.

J ørgensen $\mathrm{N}$ et al. (2001). Regional differences in semen quality in Europe. Human Reproduction, 16:1012-1019.

Lentner C (1981). Geigy scientific tables. Vol. 1: Units of measurement, body fluids, composition of the body, nutrition. Basel, Ciba-Geigy. 


\section{APPENDIX 8 National external quality control programmes for semen analysis}

Australia: Fertility Society of Australia, External Quality Assurance Schemes for Reproductive Medicine, PO Box 1101, West Leederville, Western Australia 6901, Australia

Denmark: Dansk Institut for Ekstern Kvalitetssikring for Laboratorieri, Sundhedssektoren, DEKS 54MI, Herler Universitets sygehns, Herler Ringvej 75, 2730 Herlor, Denmark

Germany: QuaDeGA, Centrum für Reproduktionsmedizin und Andrologie der Universitätsklinikum, Domagkstrasse 11, D-48129 Münster, Germany

Italy: Valutazione Esterna di Qualità, Gruppo Controllo Qualità Analitico Azienda Ospedaliero-Universitaria di Bologna, Policlinico Sant'Orsola-Malpighi, Bologna, Italy

Scandinavia: NAFA (Nordic Association for Andrology), Andrology Unit, Reproductive Medicine Centre, Karolinska Hospital, PO Box 140, SE-171 76 Stockholm, Sweden

Spain: Centro de Estudio e Investigación de la Fertilidad (CEIFER), Granada, Spain United Kingdom: UKNEQAS Schemes for Andrology, Department of Reproductive Medicine, St Mary's Hospital, Manchester M13 0J H, United Kingdom

United States of America: American Association of Bioanalysts Proficiency Testing Service, 205 West Levee, Brownsville, Texas 78520-5596, USA 
This manual is offered as a resource for scientists, technicians and managers undertaking semen analysis in clinical and research laboratories. The fifth edition provides updated, evidence-based, detailed protocols for routine, optional and research assays, with the goal of improving the quality and standardization of semen analysis and enhancing the comparability of results from different laboratories.

\section{Features of the new edition}

- An easy-to-use format that includes detailed information on each procedure;

- Additional material to explain methodology and aid in the interpretation of results;

- Numerous detailed micrographs showing examples of various sperm abnormalities;

- Sections on sperm preparation and cryopreservation;

- Evidence-based reference ranges and reference limits for various semen characteristics. 